Check for updates

Cite this: J. Mater. Chem. A, 2021, 9 , 16621

Received 11th February 2021

Accepted 25th June 2021

DOI: 10.1039/d1ta01291f

rsc.li/materials-a

\section{Tin oxide for optoelectronic, photovoltaic and energy storage devices: a review}

\author{
Goutam Kumar Dalapati, ${ }^{\text {abcde Himani Sharma }}{ }^{\mathrm{f}}$ Asim Guchhait, ${ }^{\mathrm{g}}$ \\ Nilanjan Chakrabarty, (D) ${ }^{\mathrm{h}}$ Priyanka Bamola, ${ }^{\mathrm{f}}$ Qian Liu, (DD i Gopalan Saianand, (D)j \\ Ambati Mounika Sai Krishna, ${ }^{e}$ Sabyasachi Mukhopadhyay, ${ }^{e}$ Avishek Dey, (D) \\ Terence Kin Shun Wong, ${ }^{k}$ Siarhei Zhuk, 'Siddhartha Ghosh, ${ }^{\text {e }}$ \\ Sabyasachi Chakrabortty, (D) ${ }^{m}$ Chandreswar Mahata, ${ }^{\text {n }}$ Sajal Biring, ${ }^{\text {do }}$ Avishek Kumar, ${ }^{\text {b }}$ \\ Camila Silva Ribeiro, ${ }^{\mathrm{C}}$ Seeram Ramakrishna, (D) amit K. Chakraborty, (D)*h \\ Satheesh Krishnamurthy, (D) *c Prashant Sonar (D)*ipq and Mohit Sharma (iD *r
}

Tin dioxide $\left(\mathrm{SnO}_{2}\right)$, the most stable oxide of tin, is a metal oxide semiconductor that finds its use in a number of applications due to its interesting energy band gap that is easily tunable by doping with foreign elements or by nanostructured design such as thin film, nanowire or nanoparticle formation, etc., and its excellent thermal, mechanical and chemical stability. In particular, its earth abundance and non-toxicity make it very attractive for use in a number of technologies for sustainable development such as energy harvesting and storage. This article attempts to review the state of the art of synthesis and properties of $\mathrm{SnO}_{2}$, focusing primarily on its application as a transparent conductive oxide (TCO) in various optoelectronic devices and second in energy harvesting and energy storage devices where it finds its use as an electron transport layer (ETL) and an electrode material, respectively. In doing so, we discuss how tin oxide meets the requirements for the above applications, the challenges associated with these applications, and how its performance can be further improved by adopting various strategies such as doping with foreign metals, functionalization with plasma, etc. The article begins with a review on the various experimental approaches to doping of $\mathrm{SnO}_{2}$ with foreign elements for its enhanced performance as a TCO as well as related computational studies. Herein, we also compare the TCO performance of doped tin oxide as a function of dopants such as fluorine (F), antimony (Sb), tantalum (Ta), tungsten (W), molybdenum (Mo), phosphorus (P), and gallium (Ga). We also discuss the properties of multilayer $\mathrm{SnO}_{2} /$ metal/ $\mathrm{SnO}_{2}$ structures with respect to TCO performance. Next, we review the status of tin oxide as a TCO and an ETL in devices such as organic light emitting diodes (OLEDs), organic photovoltaics (OPV), and perovskite solar cells (including plasma treatment approaches) followed by its use in building integrated photovoltaic (BIPV) applications. Next, we review the impact of $\mathrm{SnO}_{2}$, mainly as an electrode
${ }^{a}$ Center for Nanofibers and Nanotechnology, Mechanical Engineering Department, National University of Singapore, Singapore-117576. E-mail: gdalapati@gmail.com ${ }^{b}$ Sunkonnect, 1 Cleantech Loop, Singapore 637141, Singapore

${ }^{c}$ School of Engineering and Innovation, The Open University, Milton Keynes, MK76AA, UK. E-mail: Satheesh.Krishnamurthy@open.ac.uk

${ }^{d}$ Organic Electronics Research Center, Ming-Chi University of Technology, 84 Gungjuan Rd., New Taipei City 24301, Taiwan

${ }^{e}$ Department of Physics, SRM University-Andhra Pradesh, 522502, India ${ }^{f}$ Department of Physics, School of Physical Sciences, Doon University, Dehradun248001, India

${ }^{g}$ Department of Physics, P. K. College, Contai, West Bengal-721404, India ${ }^{h}$ Carbon Nanotechnology Lab, Department of Physics, Centre of Excellence in Advanced Materials, National Institute of Technology, Durgapur, Durgapur 713209, India. E-mail: amit.chakraborty@phy.nitdgp.ac.in

${ }^{i}$ School of Chemistry and Physics, Queensland University of Technology, 2 George Street, QLD 4000, Australia

${ }^{j}$ Global Centre for Environmental Remediation (GCER), College of Engineering, Science and Environment, The University of Newcastle, Callaghan, New South Wales 2308, Australia
${ }^{k}$ School of Electrical and Electronic Engineering, Nanyang Technological University, Block S2, Nanyang Avenue, Singapore 639798

${ }^{\prime}$ Empa-Swiss Federal Laboratories for Materials Science and Technology, Überlandstrasse 129, 8600 Dübendorf, Switzerland

${ }^{m}$ Department of Chemistry, SRM University-Andhra Pradesh, 522502, India ${ }^{n}$ Division of Electronics and Electrical Engineering, Dongguk University, Seoul 04620, Korea

${ }^{\circ}$ Department of Electronic Engineering, Mingchi University of Technology, New Taipei City 24301, Taiwan

${ }^{p}$ Centre for Materials Science, Queensland University of Technology, 2 George Street, QLD 4000, Australia. E-mail: sonar.prashant@qut.edu.au

${ }^{q}$ Centre for Clean Energy and Practices, Queensland University of Technology, 2 George Street, QLD 4000, Australia

'Institute of Materials Research and Engineering, $A^{*}$ STAR (Agency for Science, Technology and Research), 2 Fusionopolis Way, Innovis, \#08-03, Singapore 138634. E-mail: sharmam@imre.a-star.edu.sg 


\section{Introduction}

Metal oxide semiconductors are a class of materials which find their ever-expanding use in our life because of their interesting tunable energy band gap, excellent chemical and mechanical stability, etc. With the advancement of technologies enabling the production of metal oxides in the form of thin films, nanoparticles, nanowires and nanorods, their use has only grown over the years from semiconductor electronics to sensors, optoelectronics, catalysis, energy harvesting and storage devices. ${ }^{1-38}$ An interesting application of semiconducting metal oxides originates from the fact that some metal oxides can be doped with foreign elements such that they exhibit electrical conductivity comparable with that of metals. Thin films of such oxides allow light to pass through with negligible absorption, making such films highly desirable as electrodes for optoelectronic devices requiring materials which can both be transparent to light and conduct electricity like metals. This has led to the development of transparent conductive oxides (TCOs) which are integral to most optoelectronic and photovoltaic devices of recent times. Thin films of conducting transparent metal oxides such as $\mathrm{SnO}_{2}$ and $\mathrm{ZnO}$ (zinc oxide) are finding applications in many consumer electronic products, especially in flat panel displays, touch screen panels, photovoltaic devices, low-emissivity glasses, energysaving windows, and energy storage devices. ${ }^{\text {8-10,12-14,39 }}$ A transparent conducting film is a thin layer of electrically conductive material with low absorption (or high optical transmittance) in the visible range and is the basic requirement for any of the above devices. ${ }^{20}$ Conductivity and transparency can be customized to expand their utility in a large number of applications. $^{20-26}$ Apart from transparent conducting thin films, oxide/metal/oxide multilayer structures are also extensively studied for enhancing their optical transmittance and electrical conductivity to meet the demands as TCOs. ${ }^{\mathbf{1 1 , 4 0 - 4 2}}$ Fig. 1 shows different transparent oxides and their applications for photovoltaic devices, touch screens, flat panel displays, and energy saving smart windows. However, only a few metal oxides doped with specific elements exhibit a satisfactory performance as a TCO such as indium (In)-doped $\mathrm{SnO}_{2}$ (ITO), fluorine (F)-doped $\mathrm{SnO}_{2}$, aluminium (Al)-doped $\mathrm{ZnO}$, gallium (Ga)-doped $\mathrm{ZnO}$, etc. although each of these have their own limitations.

Tin dioxide as a transparent conducting oxide (TCO) has received huge research attention and been reviewed by several researchers due to its widespread application. .,12,43,44 $^{\mathbf{9}}$ The review articles have mostly discussed about the challenges and opportunities of ITO. It has both low electrical resistivity and facile patternability ${ }^{\mathbf{1 2 , 2 3}}$ that make it well suited for displays. However, it lacks temperature stability and resistance to chemical attack. ${ }^{10}$ Although the amount of indium is limited in the earth's crust, the human population is prone to its toxicity. ${ }^{24}$ The low-rate self-compensation effect makes it more challenging to obtain binary oxide semiconductors having a p-type conductive behavior. ${ }^{27}$ These challenges provide an impetus to seek an alternative to ITO, which has high electrical conductivity and comparable visible transmittance. To the best of our knowledge, there are no review articles which mainly focus on the metal doped $\mathrm{SnO}_{2}$ for transparent electrodes. Considering the demands of the transparent electrode for opto-electronic devices and renewable energy generation/storage, a more comprehensive review on $\mathrm{SnO}_{2}$ is needed to provide a better representation and guidance of the relevant state-of-the-art research and development.

Furthermore, an advantage of tin dioxide is that it can form oxides of different valences, which provide it with the extraordinary ability to take part in catalysis and charge storage reactions. Tin dioxide $\left(\mathrm{SnO}_{2}\right)$ is the most stable oxide of tin that finds its use not only as a TCO but also in a number of applications for sustainable development such as sensors, catalysis, energy harvesting and storage due to its earth abundance, nontoxicity and wide band gap. Naturally, a large number of

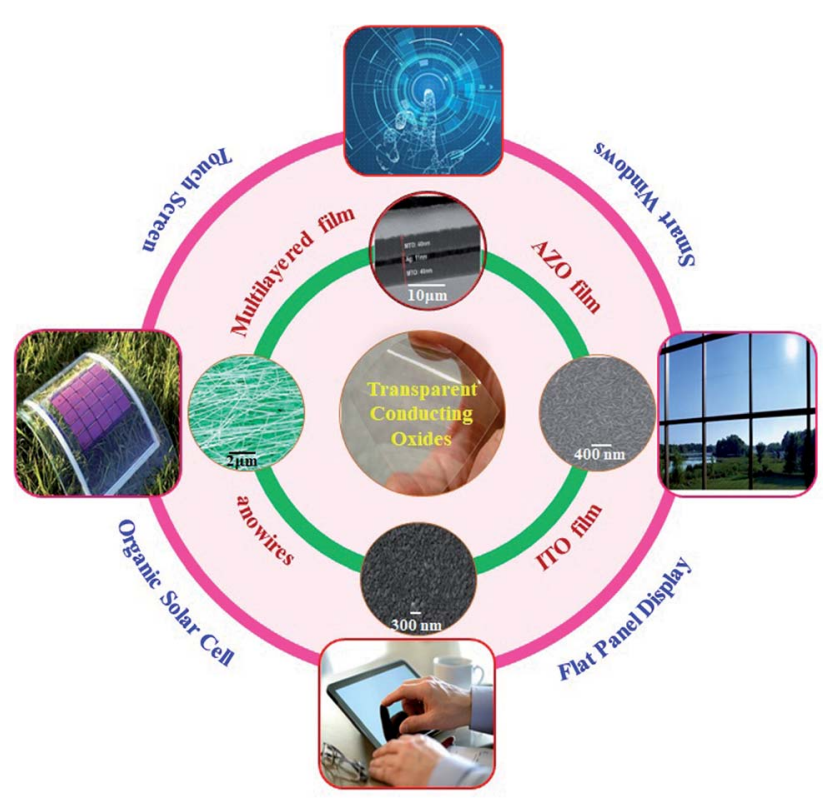

Fig. 1 Schematic diagram of various transparent conducting oxides and applications, ${ }^{45-49}$ presented with permission and copyright. 
research papers including some good review articles have already been published covering various aspects of tin oxide. For example, Das et al. ${ }^{50}$ published a comprehensive review of tin oxide, its structure and use as a gas sensor. Jenifer et al. ${ }^{\mathbf{1 1}}$ reviewed the recent advancements in tin oxide-based thin-film transistors for large-area electronics. Al Hamdi et al. ${ }^{\mathbf{2}}$ reviewed tin dioxide as a photocatalyst for water treatment. The bulk electron mobility of $\mathrm{SnO}_{2}$ is $\sim 240 \mathrm{~cm}^{2} \mathrm{~V}^{-1} \mathrm{~s}^{-1} \cdot{ }^{53} \mathrm{SnO}_{2}$ has a wide optical bandgap (3.6-4.0 eV) and a high transmittance over the entire visible regime, which indicates that when it is used in an optoelectronic device its absorption losses can be minimised. ${ }^{53}$ This wide bandgap is helpful, especially in engineering the energy levels for tandem photovoltaic devices. Other significant advantages of the $\mathrm{SnO}_{2}$ layer include chemical stability and UV-resistance properties which make $\mathrm{SnO}_{2}$ an efficient electron transport layer (ETL), especially in the case of perovskite solar cells. Earlier studies on $\mathrm{SnO}_{2}$ reveal that by alloying with metal oxides or doping with metals, its electronic properties can be selectively tuned to obtain a better optoelectronic device performance. ${ }^{54-56}$ As many as three reviews have been published within the last three years on the use of tin oxide as the ETL in perovskite solar cells (PSCs), ${ }^{53,57,58}$ in accordance with the growing interest of researchers on tin oxide as the ETL. The recent development of $\mathrm{SnO}_{2}$ as an anode for dye sensitized solar cells and its impact on the device performance have also been discussed in detail. ${ }^{59-62}$ The readers are also suggested to consult other relevant references regarding $\mathrm{SnO}_{2}$ as the ETL in organic solar cells, PSCs, and quantum dot LEDs. ${ }^{63-68}$

Deng et al. ${ }^{69}$ reviewed the development of $\mathrm{SnO}_{2}$ and graphene nanocomposites as anode materials for lithium ion batteries. However, it is clear that there is not a single review that encompassed the growing applications of $\mathrm{SnO}_{2}$ as the TCO and the ETL in optoelectronic and photovoltaic devices and as an electrode in energy storage devices. In fact, the recent developments on tin dioxide based supercapacitors and $\mathrm{Li}-$ sulfur batteries have never been reviewed to date. The review by Deng et $a .^{69}$ only focused on the tin dioxide-graphene composite as an anode material, but a review of tin oxide based composites (not only with graphene) for anode, cathode and separator is missing.

In view of this, herein, we attempt to review tin dioxide as a material with a different perspective from what has been reviewed already, i.e., a material that has enormous potential for sustainable energy applications focusing on its three major uses as a TCO, ETL and electrode, all of which strongly contribute to circular economies. The review not only tries to sum up and correlate the previous reviews on individual applications of $\mathrm{SnO}_{2}$ but also tries to cover new topics such as the impact of $\mathrm{SnO}_{2}$ for separator modification in Li-ion batteries and for mitigation of the shuttling effect by trapping polysulphides in Li-sulfur batteries. The review is organised as follows. We begin by highlighting the challenges of tin oxide as a TCO and how they are overcome by doping with different metals as well as related computational studies with special focus on earth abundant metals for sustainable applications. Various approaches used by researchers to synthesize $\mathrm{SnO}_{2}$ and doped $\mathrm{SnO}_{2}$ are also reviewed, and the TCO performances of doped tin oxide as a function of dopants such as fluorine (F), antimony (Sb), tantalum (Ta), tungsten (W), molybdenum (Mo), phosphorus (P), and gallium (Ga) are compared. The $\mathrm{SnO}_{2}$ / metal/ $/ \mathrm{SnO}_{2}$ structures with respect to TCO performance have been discussed. Next, recent advances in the use of tin oxide as the TCO and ETL in organic light emitting diodes (OLEDs), organic photovoltaics (OPV), perovskite solar cells (including plasma treatment approaches) and building integrated photovoltaic (BIPV) applications are reviewed. This is followed by a comprehensive review of the impact of $\mathrm{SnO}_{2}$, mainly as an electrode material for energy storage devices starting from the most popular Li-ion batteries to Li-sulfur batteries and finally to the rapidly emerging technology of supercapacitors, which have not been reviewed previously. Finally, we summarize the impact of $\mathrm{SnO}_{2}$ on circular economies to conclude this review.

\section{Tin oxide as a transparent conductive oxide (TCO)}

\subsection{Electronic properties of doped $\mathrm{SnO}_{2}$}

The electrical and optical properties of TCO materials are determined by their electronic structure. Mishra et $a l^{70}$ computed an energy-momentum $(E-k)$ diagram for pure stoichiometric $\mathrm{SnO}_{2}$ and Sb-doped $\mathrm{SnO}_{2}$ along several directions in $k$ space indicated by points of high symmetry within the first Brillouin zone. This band structure was computed using the augmented-spherical-wave (ASW) band-structure method for a rutile structure with a unit cell comprising two $\mathrm{SnO}_{2}$ units. The band structure of $\mathrm{SnO}_{2}$ is characterized by a single conduction band that is derived from Sn 5s-orbitals. The Snlike s character was deduced from the partial density of states function. The conduction band valley is relatively deep with the conduction band minimum (CBM) located at the $\Gamma$ point. The free electron-like dispersion can be readily seen in the $\Delta$ direction $(\Gamma-X)$ and the $\Lambda$ direction $(I-M) .{ }^{70}$ The pronounced dispersion near the $\Gamma$ point results in a large curvature for the $E$ $k$ relation and a small effective mass for conducting electrons. The experimental effective electron mass of conducting electrons in $\mathrm{SnO}_{2}$ is in the range of $0.23-0.3 m_{0}$ (depending on the direction), where $m_{0}$ is the electron mass in free space. ${ }^{71}$ This low effective mass in turn results in a higher electron mobility for conducting electrons in $\mathrm{SnO}_{2}$. By contrast, the valence band which is derived from $\mathrm{Sn}$ and $\mathrm{O}$ atomic orbitals shows much less dispersion and as a result the holes in $\mathrm{SnO}_{2}$ are generally heavy. The fundamental band gap of $3.7 \mathrm{eV}$ for $\mathrm{SnO}_{2}$ is direct and occurs at the $\Gamma$ point.

Both intrinsic point defects (oxygen vacancies) and extrinsic defects (dopants) introduce localized states in the band structure of $\mathrm{SnO}_{2}$. Kilıç and Zunger showed that oxygen vacancies in non-stoichiometric $\mathrm{SnO}_{2}$ give rise to a shallow defect level at 113 meV below the CBM. ${ }^{72}$ For Sn interstitials, the corresponding defect level is at $203 \mathrm{meV}$ above the CBM. ${ }^{71}$ Thus, both these point defects are electrically active and can contribute electrons to the conduction band. The electronic structure of $\mathrm{SnO}_{2}$ can be modified by a high dopant concentration. Mishra et al. 
computed the theoretical band structure of $\mathrm{SnO}_{2}$ doped by $\sim 8$ at $\%$ of $\mathrm{Sb}\left(\mathrm{Sn}_{3} \mathrm{SbO}_{8}\right)$ by considering a supercell consisting of two primitive unit cells stacked in the $c$ axis direction with one Sn atom substituted by a $\mathrm{Sb}$ atom..$^{70}$ The cationic dopants form an impurity $\mathrm{Sb} 5 \mathrm{~s}$ band within the band gap of $\mathrm{SnO}_{2}$ that has free electron-like dispersion, and this can directly contribute to the conductivity of $\mathrm{SnO}_{2}$. Electrons can also be excited to the next higher band derived from the Sn 5s orbitals. The CBM remains at the $\Gamma$ point and the band gap is reduced to $2.9 \mathrm{eV}$. This theoretical calculation shows that $\mathrm{Sb}$ and possibly other dopants can alter the band structure of the host $\mathrm{SnO}_{2}$. One important consequence of the strong dispersion near the CBM of $\mathrm{SnO}_{2}$ is that the Burstein-Moss (B-M) effect is readily observed in doped $\mathrm{SnO}_{2}{ }^{73}$ The B-M effect refers to an increase in the optical band gap in a semiconductor (relative to the fundamental band gap) with increasing dopant density. When the electronic states near the CBM are filled, additional energy is needed to excite electrons from the valence band to the conduction band. The B-M effect is more apparent in semiconductors with low effective electron mass.

In Fig. 2a, a comparison of conduction band alignments that have been reported in the literature is shown. Zr-doping enables up-shifting the energy level with improved band alignment which in turn increases the built in potential. ${ }^{75}$ For multivalent Sb-doping in a high concentration, the conduction band does not up-shift, but due to the oxidation of $\mathrm{Sb}$, the carrier concentration improved. ${ }^{74}$ On the other hand, Mg-doping lowers the CBM compared to un-doped $\mathrm{SnO}_{2}$ due to the reduction in free electron density in $\mathrm{SnO}_{2} \cdot{ }^{76}$ The Li-dopant in $\mathrm{SnO}_{2}$ acts as an acceptor and lowers the energy levels of $\mathrm{SnO}_{2}$ as $\mathrm{Li}^{+}$ions substitute $\mathrm{Sn}^{4+} .^{55}$ In the case of poly(vinylpyrrolidone) (PVP)-doped $\mathrm{SnO}_{2}$, the defect density decreases inside $\mathrm{SnO}_{2}$ and the electron extraction is more effective due to conduction band lowering. ${ }^{77} \mathrm{Y}$-doped $\mathrm{SnO}_{2}$ can also elevate the conduction band which leads to improved charge carrier transport. ${ }^{78} \mathrm{La}$ and $\mathrm{Zn}$ dopants are also very suitable for $\mathrm{SnO}_{2}$ which can uplift the CBM and facilitate the electron extraction and transport with less energy loss. ${ }^{79,80}$ In the case of Ga-doping into $\mathrm{SnO}_{2}$, the conduction band shifts upward with an increment of electron density and a decrement of deep traps. ${ }^{81} \mathrm{Ta}^{5+}$ doping inside the $\mathrm{SnO}_{2}$ lattice can increase the oxygen vacancy and thus the conductivity can be increased with free carrier concentration. ${ }^{82}$
It is also worth noting that the oxide thickness plays an important role in energy band gap engineering. Ke et al. demonstrated a thickness induced metal-insulator (MI) transition for epitaxially grown Sb-doped $\mathrm{SnO}_{2}$ on sapphire substrates by pulsed laser deposition. ${ }^{74} \mathrm{~A}$ critical thickness is essential for the metallic conductivity in $\mathrm{SnO}_{2}$ : $\mathrm{Sb}$ thin films (Fig. 2b). The broadening of the energy band gap as well as the enhancement of the impurity activation energy is attributed to the quantum confinement effect.

\subsection{Metal-doped tin oxide for improved transparency and conductivity}

The key challenges for $\mathrm{SnO}_{2}$ are its high resistivity and bulk defects which can trap carriers and reduce device efficiencies. As a result, selection of a suitable dopant and process design is very important to reduce bulk defects and to increase its electrical conductivity. The conductivity of $\mathrm{SnO}_{2}$ significantly depends on three critical parameters: (1) the dopant, (2) synthesis procedure, and (3) thickness of the oxide layer. ${ }^{83,84}$ In order to increase the conductivity and achieve a better performance, a different dopant may be required. Visible transmittance, conductivity, and stability of the dopant in $\mathrm{SnO}_{2}$ are very important properties for any alternative to ITO. To the best of our knowledge, a comprehensive review on $\mathrm{SnO}_{2}$ has been published by Das et al. ${ }^{85}$ and there are limited reviews that focus on doped $\mathrm{SnO}_{2}$ towards energy harvesting and storage.

Aluminium doped ZnO (AZO) and FTO are some of the other commercially available transparent conductive materials. FTO exhibits high chemical resistance, excellent thermal stability, high work function (4.9 eV), strong hardness (6.5 Mohs), ${ }^{86-88}$ and high optical transparency $(T>80 \%),{ }^{89,90}$ which make it the material of choice as TCOs for different applications. FTO has been used as a window layer in photovoltaic devices, passivation layer for energy-saving smart coatings, transparent conductor for display and flexible devices, electron transport layer, gas sensors, photodetectors, protective coatings, organic lightemitting diodes, and materials for the circular economy. ${ }^{91-97}$

Even though FTO is a promising candidate for the transparent conductor and electron transport layer, both the conductivity and mobility of FTO are still not comparable with those of ITO. Some of the key challenges affecting the performance and its electrical conductivity of the $\mathrm{SnO}_{2}$ film are the (a)
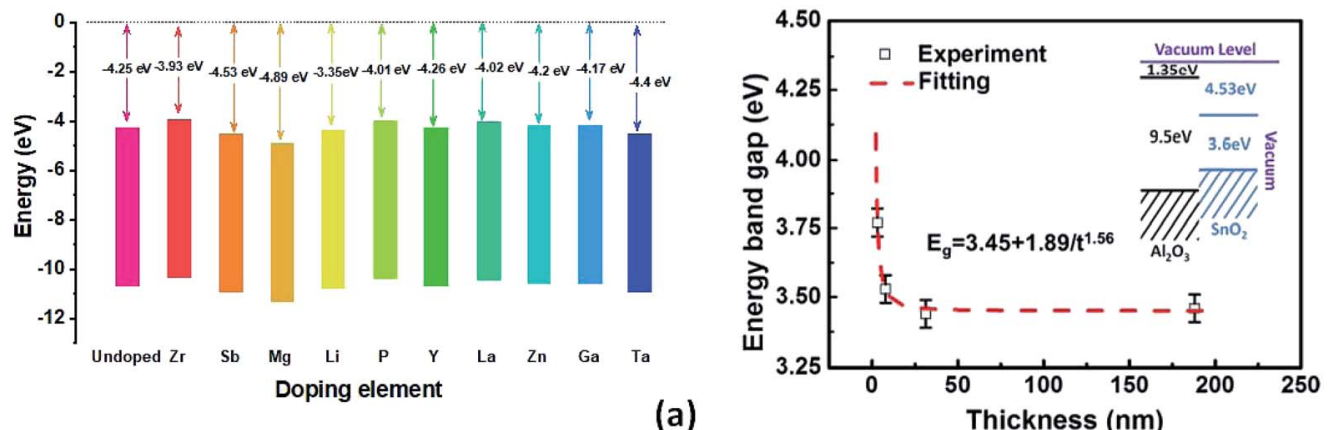

(a)

Thickness $(\mathrm{nm})$

Fig. 2 (a) Impact of doping elements on conduction band offsets. (b) Thickness-dependent energy band gap of epitaxially grown $\mathrm{Sb}$ doped $\mathrm{SnO} 2$ using pulse laser deposition, presented with permission and copyright. ${ }^{74}$ 
carrier mobility and (b) electron density. ${ }^{22,53,98,99}$ With the increase of the doping concentration, changes in electronic properties such as the modification of the bandgap, increased carrier concentration and widening of the bandgap by filling low energy levels in the conduction band are observed. As a result of this, the Fermi level shifts up towards the conduction band, causing an increase in the carrier concentration. ${ }^{\mathbf{5 4 , 9 8 , 1 0 0}}$ Several reports have discussed the electrical properties of $\mathrm{SnO}_{2}$, doped $\mathrm{SnO}_{2}$, and $\mathrm{SnO}_{2}$ based multilayer structures. ${ }^{101-111} \mathrm{Li}$ et al. reported nickel-coated FTO (Ni/FTO) through sputtering of $\mathrm{Ni}$ layers onto commercially available FTO glass and successive pulsed laser annealing under an external magnetic field (0.4 T) to enhance the electrical performance of FTO. ${ }^{103}$ Similarly, Chen et al. demonstrated a non-thermal dual-plasma synthesis for antimony doped $\mathrm{SnO}_{2}, \mathrm{Sb}-\mathrm{SnO}_{2}$ (ATO) nanocrystals with a uniform composition and a conductivity of $0.1 \mathrm{~S} \mathrm{~cm}^{-1}$ over a high surface area. ${ }^{112}$ While these methods show an increase in performance, the morphology is affected due to the presence of pinholes after thermal treatment. The lanthanum (La) dopant is a very promising alternative capable of alleviating $\mathrm{SnO}_{2}$ crystal aggregation and it provides a platform with full coverage and helps to form a homogeneous film. ${ }^{80}$ Furthermore, the La dopant reduces the band offset of the $\mathrm{SnO}_{2}$ layer with increased electron extraction and suppressing charge recombination and thus enhances the power conversion efficiency from $14.24 \%$ to $17.08 \%$ for perovskite-based solar cells. ${ }^{80}$

In modern technology, $\mathrm{SnO}_{2}$ based TCOs play a central role in optical and electronic applications. The performance of these devices depends critically on the dopant and the properties of $\mathrm{SnO}_{2}$. Apart from indium (In), doping of tin oxide can be realized with various elements, such as antimony ( $\mathrm{Sb})$, fluorine $(\mathrm{F})$, niobium ( $\mathrm{Nb})$, tungsten $(\mathrm{W})$, phosphorus $(\mathrm{P})$, strontium $(\mathrm{Sr})$, tantalum (Ta), lanthanum (La), lithium (Li), gallium (Ga), molybdenum (Mo), and cobalt (Co) as these dopants provide precise control over its electrical and optical properties. $^{\mathbf{7 1 , 8 1 , 1 0 1 - 1 0 4 , 1 1 3 - 1 3 0}}$ All these dopants are discussed in this section. For example $\mathrm{Sb}$ and $\mathrm{F}$ are the most suitable dopants for $\mathrm{SnO}_{2},{ }^{\mathbf{3 0 , 1 0 1 - 1 0 4 , 1 1 4}}$ wherein $\mathrm{Sb}$ substitutes $\mathrm{Sn}$ atoms and $\mathrm{F}$ substitutes $\mathrm{O}$ atoms. ${ }^{89} \mathrm{Sb}$ is an effective dopant because the $\mathrm{Sb}^{5+}$ and $\mathrm{Sn}^{4+}$ ions are of similar radii. In the case of $\mathrm{Sb}$ doped tin oxide (ATO), it has high transparency $(\sim 80 \%)$ and low resistivity $\left(\sim 10^{-3} \Omega \mathrm{cm}\right)$, good mechanical hardness, and environmental stability. ${ }^{88}$ One key feature of ATO is that its carrier density increases monotonically with $\mathrm{Sb}$ doping within the range of $10^{20}$ $\mathrm{cm}^{-3}{ }^{86}$ while the dopant activation efficiency decreases from $60 \%$ to $20 \%$. The room temperature Hall mobility of ATO ranges from $6 \mathrm{~cm}^{2} \mathrm{~V}^{-1} \mathrm{~s}^{-1}$ to $24 \mathrm{~cm}^{2} \mathrm{~V}^{-1} \mathrm{~s}^{-1}$.

FTO is a stable $\mathrm{TCO}^{\mathbf{1 1 5 , 1 1 6}}$ that has low electrical resistivity due to the high carrier density. ${ }^{89}$ It can strongly adhere to any substrate making it suitable for device integration. ${ }^{16}$ However, its electrical conductivity is not as high as niobium (Nb)-doped tin oxide (NbTO) films. ${ }^{117}$ Niobium is an exceptional dopant for $\mathrm{SnO}_{2}$ as the ionic radius of $\mathrm{Nb}^{5+}(0.064 \mathrm{~nm})$ matches that of $\mathrm{Sn}^{4+}$ $(0.069 \mathrm{~nm})$, which enables the substitution of $\mathrm{Nb}^{5+}$ for $\mathrm{Sn}^{4+}$ in the $\mathrm{SnO}_{2}$ crystal lattice structure. ${ }^{118}$ If $\mathrm{SnO}_{2}$ is concurrently doped with $\mathrm{Nb}$ and $\mathrm{F}$ to replace the $\mathrm{Sn}^{4+}$ and $\mathrm{O}^{2-}$ in the $\mathrm{SnO}_{2}$ lattice, respectively, its Hall mobility and carrier generation will be further enhanced, making it suitable for high efficiency devices. ${ }^{118}$ The substitution of $\mathrm{Nb}^{5+}$ for $\mathrm{Sn}^{4+}$ and the integration of $\mathrm{F}^{-}$are beneficial in improving the overall performance. The synergistic effect of $\mathrm{Nb}$ and $\mathrm{F}$ co-doped $\mathrm{SnO}_{2}$ films results in improved optoelectronic properties compared to those of $\mathrm{F}$ or Nb-doped $\mathrm{SnO}_{2}$ films. ${ }^{118}$ Nitrogen is another low-cost and environmentally friendly dopant for $\mathrm{SnO}_{2}$. The nitrogen dopant reduces the optical energy threshold and enhances the film conductivity. ${ }^{115}$

Recently, molybdenum (Mo) and tungsten (W) doped $\mathrm{SnO}_{2}$ have been studied by Huo et al. ${ }^{119}$ The Mo-doped and W-doped $\mathrm{SnO}_{2}$ films show an average transmittance of $\sim 60 \%$ over a wavelength range between 300 and $2500 \mathrm{~nm}$, which is $\sim 2$ times higher compared to ITO films. ${ }^{119}$ Tungsten is an important cation dopant for the $\mathrm{SnO}_{2}$ based transparent conductive material. The electronic and optical properties of $\mathrm{SnO}_{2}$ can be enhanced by replacing $\mathrm{Sn}^{4+}$ through $\mathrm{W}^{6+} \cdot{ }^{125}$ Moreover, $\mathrm{W}^{6+}$ has the highest valence state among the common doping elements to generate more free electrons. ${ }^{125}$ Doping with $\mathrm{W}$ is also very helpful to generate more charge carriers and maintain the structural ability of $\mathrm{SnO}_{2} \cdot{ }^{126}$ Thus, W doped $\mathrm{SnO}_{2}$ might solve the problems related to $\mathrm{SnO}_{2}$ based anode materials for lithiumion batteries due to its unique characteristics. ${ }^{125}$ Doping of strontium ( $\mathrm{Sr}$ ) in $\mathrm{SnO}_{2}$ further widens its potential in improving the photocatalytic activity, iteration of the electronic structure, and enhancement of vital physical and chemical properties. ${ }^{127}$ Studies influencing the third-order nonlinear optical properties of $\mathrm{Sr}$-doped $\mathrm{SnO}_{2}$ were limited, and further elucidation is required for its applications in various optical devices. ${ }^{127}$ In another study, Bannur et al. observed a third-order nonlinear absorption mechanism for $\mathrm{Sr}: \mathrm{SnO}_{2}$ films, which is attributed to free carrier absorption induced two-photon absorption. ${ }^{127}$ The third-order nonlinear absorption co-efficient $\left(\beta_{\text {eff }}\right)$ shows one order of improvement $\left(0.14 \times 10^{-1} \mathrm{~cm} \mathrm{~W}^{-1}\right.$ to $3.91 \times 10^{-1} \mathrm{~cm}$ $\mathrm{W}^{-1}$ ), which indicates the competency of grown films in nonlinear optical device applications. ${ }^{\mathbf{1 2 7}}$

He et al. demonstrated the optical and structural properties of Ta-doped $\mathrm{SnO}_{2}$ monocrystal films grown on $\mathrm{MgF}_{2}$ (110) substrates. The Ta dopant for $\mathrm{SnO}_{2}$ takes the form of $\mathrm{Ta}^{5+}$ ions. ${ }^{128}$ Ta-doped $\mathrm{SnO}_{2}$ films are of importance due to the high work function of $5.2 \mathrm{eV}$, with an average transmission over $87 \% .{ }^{128}$ Ta doping for $\mathrm{SnO}_{2}$ films show reduced resistivity and improved Hall mobility. ${ }^{128}$ The highest Hall mobility of $74.2 \mathrm{~cm}^{2}$ $\mathrm{V}^{-1} \mathrm{~s}^{-1}$ is attained for the 5 at\% Ta doped $\mathrm{SnO}_{2}$ film, and the least resistivity $2.5 \times 10^{-4} \Omega \mathrm{cm}$ is attained at 6 at\% Ta doping. ${ }^{128}$ It is worth noting that a Ta-doped $\mathrm{SnO}_{2}$ transparent conductive oxide has been demonstrated as a selectively solar transmitting coating for the high temperature concentrating solar power technology. ${ }^{129}$

Apart from the above-discussed materials, $\mathrm{SnO}_{2}$ can also be doped using aluminium ${ }^{\mathbf{1 2 2 - 1 2 4}}$ to achieve p-type conductivity, ${ }^{\mathbf{1 2 0}}$ along with annealing at an elevated temperature of $450{ }^{\circ} \mathrm{C}$. In such a process, the electron concentration decreases with aluminum doping due to substitution effects. Gallium (Ga) is another promising p-type dopant for $\mathrm{SnO}_{2}$ since it can substitute $\mathrm{Sn}$ atoms with less lattice deformation compared to $\mathrm{Al}$ and In. ${ }^{27}$ The doping of Ga is quite effective in the context of the role 
of $\mathrm{SnO}_{2}$ in perovskite-based devices. Ga-doped $\mathrm{SnO}_{2}$ is used as an efficient electron transport layer in planar perovskite solar cells (PSCs) ${ }^{81}$ because of its better band alignment with the perovskite absorption layer for efficient electron extraction. ${ }^{81}$ Besides, Ga doping reduced the trap state density in $\mathrm{SnO}_{2}$, leading to a lower recombination and negligible hysteresis. ${ }^{81}$ All these combined to deliver an improved efficiency. Several other dopants in $\mathrm{SnO}_{2}$ that can also improve the PSC performance will be discussed below in the section 'Impact of $\mathrm{SnO}_{2}$ on PSCs'. Cobalt (Co) is also a good substitute for noble metals as the dopant for $\mathrm{SnO}_{2}$. Co ions can easily replace tin ions in $\mathrm{SnO}_{2}$ without destroying the lattice structure because of the compatible ionic radii. ${ }^{130}$ Also, the Co dopants can enter the tetragonal rutile type $\mathrm{SnO}_{2}$ and suppress the grain growth. ${ }^{\mathbf{1 3 0}}$
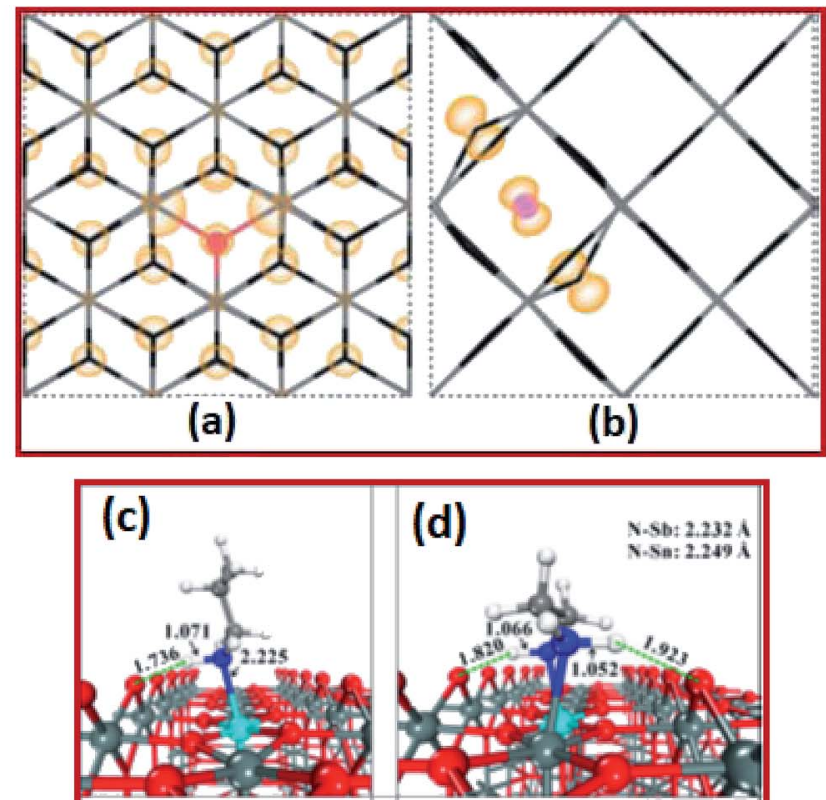

(e)
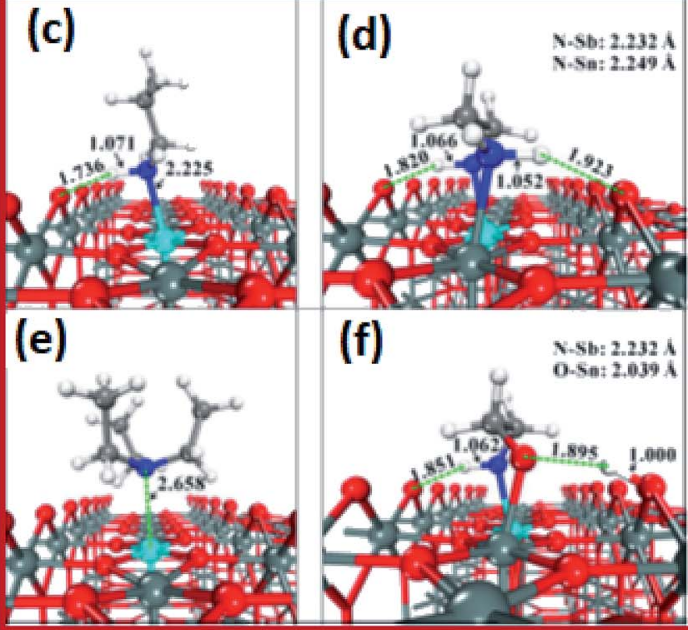

(f)

N.5b: $2732 A$

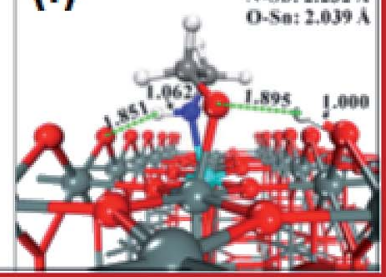

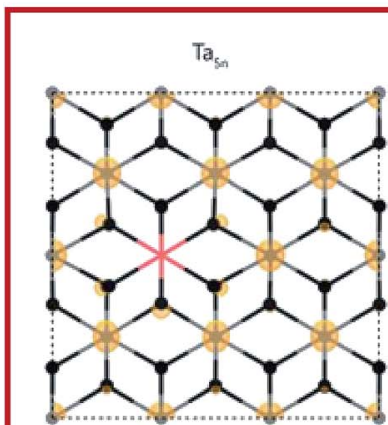

(g)

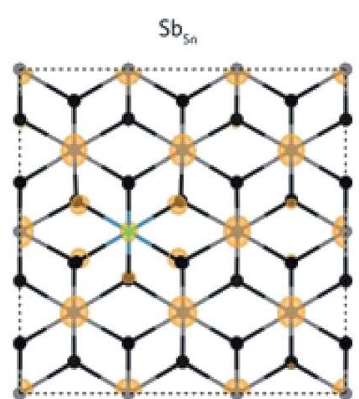

(h)

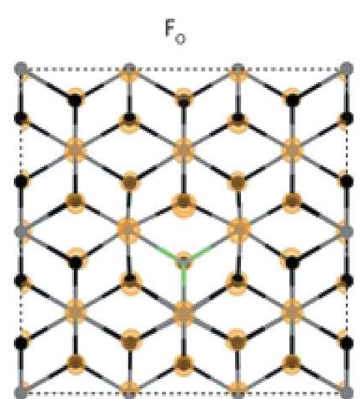

(i)

Fig. 3 Calculated partial charge densities of (a) $\mathrm{FO}^{+}$and (b) $\mathrm{Fi}^{-}$in the down and across directions, respectively. The $\mathrm{Sn}$ (gray) and $\mathrm{O}$ (black) atoms are depicted using a stick model for clarity, while the $\mathrm{F}$ atoms are colored red (FO) and pink (Fi) corresponding to the defect color. Charge densities of 0.001 and $0.02 \mathrm{eV} \AA^{-1}$ were used for panels (a) and (b), respectively, ${ }^{22}$ presented with permission and copyright. The preferred adsorption configurations of (c) propylamine (PA), (d) ethylenediamine (EDA), (e) triethylamine (TEA), and (f) monoethanolamine (MEA) on the ATO (110) surface. The bond distances are given in A. Color codes: O-red, Sn-gray green, Sb-cyan, C-gray, N-blue, and H-white, ${ }^{106}$ presented with permission and copyright. The partial charge densities at the $\mathrm{CBM}_{\text {of }} \mathrm{SnO}_{2}$ for TaSn (g), SbSn (h), and FO (i). The densities highlight the fact that $\mathrm{Sb}$ and $\mathrm{F}$ both hybridize with the $\mathrm{CBM}$, thus having a detrimental effect on the band curvature with increased doping concentrations, and that Ta does not undergo this same effect, ${ }^{107}$ presented with permission and copyright. 
The doping in $\mathrm{SnO}_{2}$ results in the modification of optical and electronic properties that are needed for various applications.

\subsection{Design of doped $\mathrm{SnO}_{2}$ for TCO applications: computational modeling}

The computational screening approach for dopants of $\mathrm{SnO}_{2}$ comprises an efficient and reliable calculation of its bandgap, effective mass, binding energy, and the formation energy. ${ }^{12,100,131-133}$ In recent times, computational screening approaches have shown immense potential in identifying suitable dopants of $\mathrm{SnO}_{2}$ to increase the inherent electrical and optical properties. Towards this end, Cheng et al. investigated the first-principles-based computer screening system in search of suitable dopants or co-dopants for $\mathrm{SnO}_{2}$ to develop new $\mathrm{SnO}_{2}$ based transparent conducting oxide systems. ${ }^{100}$ Based on computer simulations, it is found that the best candidates for $\mathrm{SnO}_{2}$-based TCO materials are FTO, ATO, phosphorus (P) doped $\mathrm{SnO}_{2}$ PTO (P-doped $\mathrm{SnO}_{2}$ ) and FPTO (F and P co-doped $\left.\mathrm{SnO}_{2}\right){ }^{100}$

In another interesting work, Swallow et al. investigated the ntype FTO $\left(n>10^{20} \mathrm{~cm}^{-3}\right)$ deposited onto soda-lime glass via atmospheric pressure chemical vapor deposition (APCVD) to reveal inherent self-compensation, which limits the mobility, achievable free electron density, and higher conductivity. ${ }^{22}$ By using DFT energy calculations, it is determined that the interstitial fluorine in the -1 charge state might be the lowest formation energy acceptor defect for degenerately doped FTO, as shown in Fig. 3a and b. ${ }^{22}$ Hence, they provided new confirmation of fluorine interstitial as the defect responsible for FTO falling well short of the theoretical ionized impurity scattering with a limited mobility of $>100 \mathrm{~cm}^{2} \mathrm{~V}^{-1} \mathrm{~s}^{-1} .{ }^{22}$

ATO is more advantageous due to its low cost and abundance. ATO thin films also display excellent electronic and optical properties comparable to those of ITO films and thus ATO is emerging as a promising alternative to ITO. Borgatti et al. elucidated the origin of the satellite structure observed in the Sn $4 \mathrm{~d}$ core-level photoemission spectrum (PES) of ATO by comparing the experimental measurements to results obtained from $a b$ initio many-body perturbation theory. ${ }^{134}$ They established that such a satellite structure is produced by the coupling of Sn $4 \mathrm{~d}$ core electrons to the plasma oscillation of the free electrons observed in the material through doping. ${ }^{.134}$ Moreover, within the same theoretical framework, the enrichment of the asymmetric tail from the valence band photoemission spectrum of doped $\mathrm{SnO}_{2}$ was also explained. ${ }^{134}$ These results reveal that to capture the satellite structures for narrow-band materials and to identify properly the underlying electronic structure excitations, it is vital to go beyond the homogeneous electron gas (HEG) electron-plasmon coupling model and to perform materialspecific ab initio calculations. ${ }^{134}$ The GW (where G is the oneparticle Green's function and $\mathrm{W}$ is the screened coulombic interaction) approximation (GWA) for the self-energy and the cumulant (C) expansion of the Green's function were incorporated into the first-principle $\mathrm{GW}+\mathrm{C}$ scheme to interpret the electron correlation in PES spectra. In this perception, the results for ATO imply that the GW + C theory can be a very promising approach for the interpretation of electron correlation features for PES of several conductive oxide materials. ${ }^{134} \mathrm{Kim}$ et al. investigated the electronic structure of pure and doped $\mathrm{SnO}_{2}$ nanocrystals within a range of 1.3-2.4 nm diameter. Herein, strong quantum confinement effects were observed and the electron binding energy for Sb doped nanocrystals decreases with the size. ${ }^{135}$

In another study, Chen et al. investigated an instant postsynthesis strategy for aqueous colloidal dispersions of nanocrystals, using ethylenediamine (EDA), propylamine (PA), monoethanolamine (MEA), and triethylamine (TEA). ${ }^{136}$ By using DFT calculations, they found strong attractive interactions between amines and ATO surfaces via $\mathrm{N}-\mathrm{Sn}$ and especially $\mathrm{N}-\mathrm{Sb}$ bonding interactions, as shown in Fig. $3 \mathrm{c}-\mathrm{f} .{ }^{136}$ The energies of amine adsorption on the Sb site vary from $0.95 \mathrm{eV}$ to $3.28 \mathrm{eV}$, following the order of TEA $<$ PA $<$ EDA $<$ MEA, which is at least $0.2 \mathrm{eV}$ higher than the corresponding adsorptions of the $\mathrm{Sn}$ site. ${ }^{136}$ This implies stronger adsorption of amines on Sb sites than on Sn sites. The proposed strategy improved the performance of electrochromic devices such as good reversibility, fast response, and high optical modulation. ${ }^{136}$ Williamson et al. demonstrated that tantalum (Ta) is a resonant donor in $\mathrm{SnO}_{2}$ using a combination of hybrid DFT calculations, IR reflectivity, and hard X-ray photoelectron spectroscopy ${ }^{137}$ It is reported that Ta is a superior dopant to both fluorine and antimony (Fig. 3gi), with the capacity to yield higher conductivity, mobility, and better IR transparency as compared to FTO and ATO. ${ }^{137}$ These findings imply that Ta-doped $\mathrm{SnO}_{2}$ has the potential for large surface area applications with low-cost TCO substrates. ${ }^{137}$

Ganose $e t$ al. used DFT to show that incorporation of lead $(\mathrm{Pb})$ into $\mathrm{SnO}_{2}$ reduces the bandgap through lowering of the conduction band minimum, thereby increasing the electron affinity. ${ }^{54}$ The electron effective mass at the conduction band minimum decreases alongside the bandgap, demonstrating an improved charge carrier mobility. ${ }^{54}$ Moreover, the calculated optical absorption properties show that the alloys maintain their transparency in the visible spectrum. These properties make $\mathrm{SnO}_{2}: \mathrm{Pb}$ a more efficient $\mathrm{n}$-type transparent material and an ideal candidate for use in TCO applications. ${ }^{54}$

Phosphorus (P)-doped $\mathrm{SnO}_{2}\left(\mathrm{SnO}_{2}: \mathrm{P}\right)$, PTO, films were synthesized by an aerosol assisted chemical vapor deposition route with excellent optical and electrical properties. ${ }^{5} \mathrm{~A}$ data generator was used to build computational models of $\mathrm{P}$ as a dopant for $\mathrm{SnO}_{2}$ and showed that phosphorus acts as a shallow one electron n-type donor allowing improved conductivities. P does not suffer from self-compensation issues associated with other dopants, such as F. This synthetic route opens up the possibility of using a common element to dope $\mathrm{SnO}_{2}$ films for transparent conducting oxide applications. ${ }^{5}$

\section{Design of doped $\mathrm{SnO}_{2}$ for $\mathrm{TCO}$ applications: different experimental approaches}

As discussed in the previous section, the conductivity of undoped $\mathrm{SnO}_{2}$ is significantly improved by doping with various dopant elements such as F, ${ }^{103,104} \mathrm{Mn},{ }^{105,138} \mathrm{Ta},{ }^{106,107}$ and Sb. ${ }^{101,102}$ 


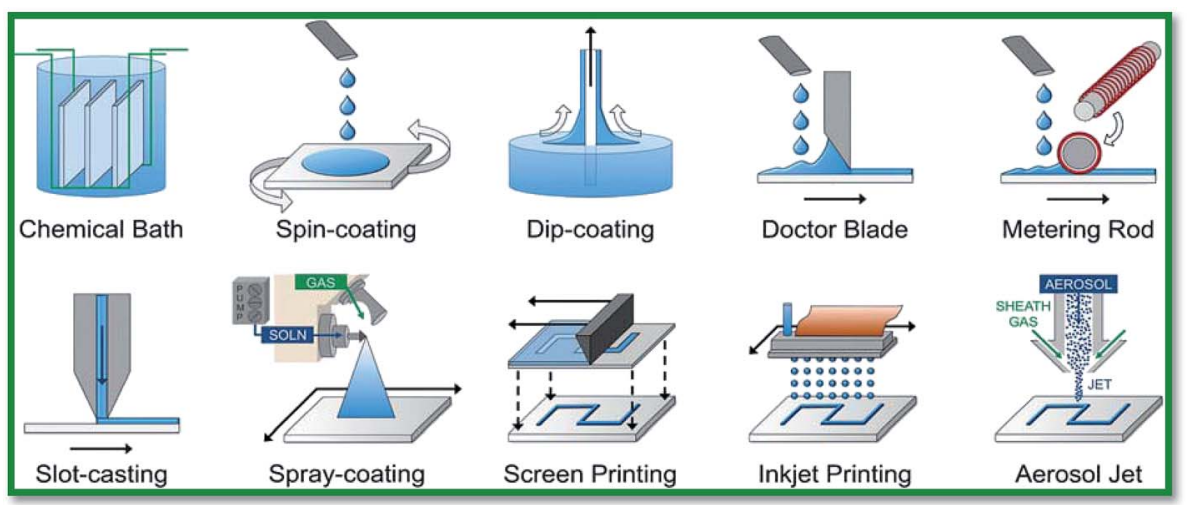

Fig. 4 Various solution-based approaches for metal oxide film deposition. Reproduced with permission. ${ }^{139}$ Copyright 2011 , The Royal Society of Chemistry.

Tin oxide thin films are usually deposited using solution-based deposition and vacuum-based deposition techniques. Solutionbased deposition methods offer numerous benefits over vacuum-based deposition techniques; e.g. simpler processing, better scalability, and lower manufacturing $\operatorname{cost}^{139,140}$ On the other hand, thin films grown using solution-based techniques have porous surface morphologies, and their electrical properties are relatively poor compared to those of vacuum-based techniques. Several solution-based approaches such as the sol-gel process, hydrothermal synthesis, chemical bath deposition, successive ionic layer adsorption and reaction, and spray deposition are widely reported for doping $\mathrm{SnO}_{2}$ films. To this end, Pasquarelli and co-workers have thoroughly reviewed several solution-based film deposition processes, as elucidated in Fig. $4 .{ }^{139}$ In this review article, we discuss the doping of $\mathrm{SnO}_{2}$ using solution and vacuum based techniques. Synthesis of high quality doped $\mathrm{SnO}_{2}$ is much sought-after. The impact of dopants on its surface morphology and optical and electronic properties is discussed in detail.

\subsection{Sol-gel and spin coating}

Due to its simplicity, sol-gel based processes have gained popularity, and the sol-gel deposition of doped $\mathrm{SnO}_{2}$ films is already well established. ${ }^{141,142}$ A facile approach has been reported to control the morphology of doped and pure $\mathrm{SnO}_{2}$ films by a sol-gel dip-coating process. ${ }^{143}$ The root mean square (RMS) roughness value of $\mathrm{SnO}_{2}$ :Sb thin films was found to be $1 \%$ of film thickness, which makes them suitable for optoelectronic applications. The authors reported a resistivity of $\sim 10^{-5} \Omega \mathrm{m}$ for the $5 \mathrm{~mol} \% \mathrm{Sb}$-doped $\mathrm{SnO}_{2}$ films. An average transmittance of $>80 \%$ (in the UV-vis region) was found for all these films. The bandgap energy of $\mathrm{SnO}_{2}: \mathrm{Sb}$ varies in the range of 3.69-3.97 eV with an increase in Sb doping concentration. ${ }^{143}$

Shi et al. studied the effect of fluorine concentration on FTO films by the improved sol-gel method. ${ }^{144}$ The surface morphology, optical properties, and electrical properties of films were investigated for different fluorine concentrations. The grain size increases with an increase in $F$ concentration, as shown in Fig. 5a-f. The particle shape changes from a rod-like structure to a pyramid with an increase of $F$ concentration. This is particularly beneficial for energy conversion devices. The visible transmittance and sheet resistance significantly depend on the F concentration (Fig. $5 g$ and $h$ ). The sheet resistance for un-doped $\mathrm{SnO}_{2}$ films is $450 \Omega \mathrm{sq}^{-1}$. However, the sheet resistance decreases from $450 \Omega \mathrm{sq}^{-1}$ to $14.7 \Omega \mathrm{sq}^{-1}$ with the increase of fluorine concentration. ${ }^{144}$

In another study, it is observed that with a lesser number of coatings, the film has larger inter-grain boundaries (i.e. more porous) leading to poor electrical properties. A large number $(>7)$ of sol-layers lead to cracks in the film that decreases the Hall mobility. ${ }^{140}$ Therefore, an optimum thickness is required to obtain a higher electrical conductivity that, in turn, gives the preferred dense surface morphology for better electrical performance. Jin $e t$ al. demonstrated an improved approach in which stannous oxalate $\left(\mathrm{SnC}_{2} \mathrm{O}_{4}\right)$ was dispersed in deionized (DI) water together with citric acid and triethanolamine to obtain a sol-gel solution. The dip-coated film shows a lower sheet resistance of $\sim 30-40 \Omega \mathrm{sq}^{-1}$. $^{145}$ Doping of Ta and $\mathrm{Nb}$ in $\mathrm{SnO}_{2}$ films by the dip-coating technique achieves moderate electrical properties. ${ }^{146}$

Gallium (Ga)-doped $\mathrm{SnO}_{2}$ semiconductors show p-type conductivity with an average optical transmittance of more than $87 \%$. Thin film Ga-doped $\mathrm{SnO}_{2}$ was fabricated using a solgel spin coating process with a doping concentration of gallium greater than $10 \% .{ }^{27} \mathrm{Ga}$ doping reduces the grain size from $7.63 \mathrm{~nm}$ to $3.36 \mathrm{~nm}$ as the Ga doping concentration increased from $0 \%$ to $20 \%$. The RMS surface roughness increased from $2.34 \mathrm{~nm}$ at $0 \%$ doping to $1.29 \mathrm{~nm}$ at $20 \%$ and the band gap energy decreased from $3.92 \mathrm{eV}$ for undoped to $3.83 \mathrm{eV}$ for $15 \%$ Ga doping. The highest mean hole concentration obtained by this method $\left(1.70 \times 10^{18} \mathrm{~cm}^{-3}\right)$ is slightly lower than that obtained by DC (direct current) magnetron sputtering $\left(8.84 \times 10^{18}\right.$ $\mathrm{cm}^{-3}$ ), as reported by Huang et al. ${ }^{147}$ Moreover, the carrier mobility was found to decrease as the resistivity increased with doping. An organic additive-free aqueous solution based process was reported for the sol-gel synthesis of doped $\mathrm{SnO}_{2}$. Film formation was demonstrated via an evaporation-driven method and dip-coating in a thermostatic oven at $25-60{ }^{\circ} \mathrm{C}$. A crystalline $\mathrm{SnO}_{2}$ film was obtained after heat treatment at 

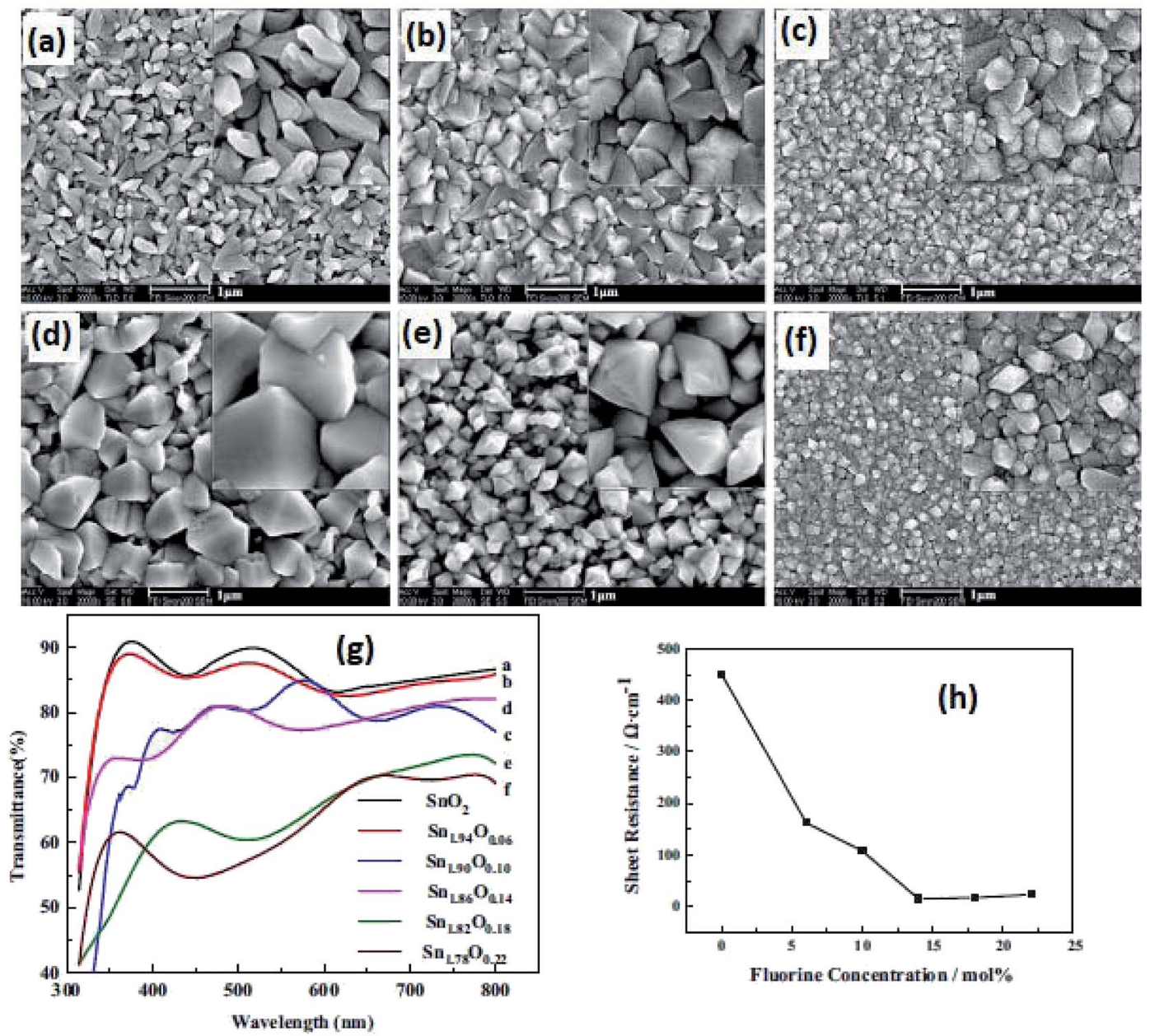

Fig. 5 SEM images of films with different $\mathrm{F}$ concentrations: (a) $\mathrm{SnO}_{2}$, (b) $\mathrm{SnO}_{1.94} \mathrm{~F}_{0.06}$, (c) $\mathrm{SnO}_{1.9} \mathrm{~F}_{0.10}$, (d) $\mathrm{SnO}_{1.86} \mathrm{~F}_{0.14}$, (e) $\mathrm{SnO}_{1.82} \mathrm{~F}_{0.18}$, and (f) $\mathrm{SnO}_{1.78} \mathrm{~F}_{0.22}$. (g) Transmission spectra of films with different $\mathrm{F}$ concentrations and (h) sheet resistance of $\mathrm{SnO}_{2}$ films with different $\mathrm{F} / \mathrm{Sn}$ ratios, ${ }^{144}$ presented with permission and copyright.

$700{ }^{\circ} \mathrm{C}$ for 10 min. ${ }^{148}$ Non-toxic stannous fluoride $\left(\mathrm{SnF}_{2}\right)$ was also used as a fluorine source to deposit the fluorine-doped $\mathrm{SnO}_{2}$ thin films. The process was referred to as the green sol-gel method. The $\mathrm{SnF}_{2}$ content was varied from 0 to $10 \mathrm{~mol} \%$ to optimise differing conductivity. The solution was stirred at $80{ }^{\circ} \mathrm{C}$ for $2 \mathrm{~h}$ and then dip-coated to form FTO thin films, which exhibited a resistivity around $7.0 \times 10^{-4} \Omega \mathrm{cm} .{ }^{149}$

Film deposition using $\mathrm{SnO}_{2}$ nanoparticles is similar to the sol-gel process and involved pre-synthesized nanoparticles. This process has the advantage that post-deposition annealing at high temperatures is not necessary to achieve crystalline films because nanoparticles are already in the crystalline phase. In another report, Zhao et al. demonstrated a surfactant-free and binder-free deposition of $\mathrm{Sb}: \mathrm{SnO}_{2}$ in a compact thin film using $\mathrm{Sb}: \mathrm{Sn}_{3} \mathrm{O}_{4}$ suspension. ${ }^{150}$ The as-prepared films have electrical resistivity around $3.04 \times 10^{-2} \Omega \mathrm{cm}$ and a transparency of $\sim 92.70 \%{ }^{150}$ Synthesis of highly crystalline $\mathrm{Sb}-\mathrm{SnO}_{2}$ nanoparticles is achieved by microwave heating of the antimony acetate and $\mathrm{SnCl}_{4}$ precursors in benzyl alcohol and toluene at $135{ }^{\circ} \mathrm{C}$ for $15 \mathrm{~min}$. The spin-coating of ATO dispersion resulted in uniform film deposition with $90 \%$ transparency and $1.9 \times$
$10^{-2} \Omega$ cm resistivity. ${ }^{151}$ Deposition of F-doped $\mathrm{SnO}_{2}$ films by Nadarajah et al. involved a reactive tin(II) hydroxide nitrate nanoscale cluster in an aqueous solution. Those films rendered very low electrical resistivity $\left(1.5 \times 10^{-4} \Omega \mathrm{m}\right)$ and optical transmittance $(>85 \%) .{ }^{152}$ It is also worth noting that, generally, dopants which formed nanoparticles resulted in a rough surface.

\subsection{Chemical bath deposition}

A low temperature and single precursor based chemical bath deposition (CBD) approach was presented by Tsukuma et al. ${ }^{\mathbf{1 5 3}}$ in which $\mathrm{SnF}_{2}$ was dissolved in DI water, and film growth occurred at $40{ }^{\circ} \mathrm{C}$. After the heat treatment, the electrical properties improved significantly and the resistivity was measured to be $\sim 1.4 \times 10^{-2} \Omega \mathrm{cm} .{ }^{153}$ An aqueous solution of $\mathrm{SnF}_{2}$ and $\mathrm{HF}$ resulted in a thin film when small amounts of $\mathrm{H}_{2} \mathrm{O}_{2}$ and/or $\mathrm{H}_{3} \mathrm{BO}_{3}$ were added, but the resistivity was found to be $\sim 18.7 \Omega \mathrm{cm} .{ }^{154}$ This high resistivity is due to poor crystallinity and the presence of high interface defects for the film synthesized by using CBD and liquid phase deposition (LPD) methods 
at low temperatures $\left(40-75{ }^{\circ} \mathrm{C}\right) .{ }^{154}$ Raviendra et al. ${ }^{155}$ demonstrated an electroless deposition of polycrystalline $\mathrm{Sb}-\mathrm{SnO}_{2}$ thin films using stannic chloride $\left(\mathrm{SnCl}_{4}\right)$, ammonium fluoride $\left(\mathrm{NH}_{4} \mathrm{~F}\right)$, and silver nitrate in a solution at room temperature. The visible transmittance and reflectance in the infra-red region of pristine $\mathrm{SnO}_{2}$ films were found to be $\sim 80 \%$ and $\sim 70 \%$, respectively, with resistivity on the order of $\sim 10^{-2} \Omega \mathrm{cm}$. In contrast, $\mathrm{Sb}$-doped $\mathrm{SnO}_{2}$ films showed a visible transmittance of $86 \%$ and an infra-red reflectance of $\sim 83 \%$ with resistivity in the range of $\sim 10^{-3}-10^{-4} \Omega \mathrm{cm}$. The resistivity of the $\mathrm{Sb}$-doped thin films was excellent and comparable to the films deposited using the physical deposition process. When the antimony doping concentration was increased from 0 to 5 at\%, the grain size increased from 30 to $65 \mathrm{~nm}$. The larger grain size reduced the grain boundary scattering by reducing the grain boundary potential, which resulted in enhanced mobility and conductivity. ${ }^{155}$ However, this process required fine control over $\mathrm{pH}$ and therefore reproducibility can be challenging. ${ }^{155}$

\subsection{Spray coating and aerosol jet}

Spray pyrolysis is a widely used deposition technique for achieving high-quality pure and doped $\mathrm{SnO}_{2}$ films on a hot glass substrate $\left(400-600{ }^{\circ} \mathrm{C}\right)$. This process is simple, inexpensive and efficient. ${ }^{\mathbf{8 7} \mathbf{1 5 6 , 1 5 7}}$ It makes the process of adding several dopants easier with a high growth rate and reproducibility and enables mass customization for homogeneous large part deposition. ${ }^{158-160}$ However, due to high processing temperatures, it can result in certain constraints in depositing the top electrode on functional layers. In these deposition processes, the sheet resistance initially decreases from $189.0 \Omega \mathrm{sq}^{-1}$ to a minimum of $4.1 \Omega \mathrm{sq}^{-1}$ with the increase of the substrate temperature from $250{ }^{\circ} \mathrm{C}$ to $300{ }^{\circ} \mathrm{C}$ and then saturates. ${ }^{158} \mathrm{In}$ this process, the lattice parameters remain fairly constant with temperature but the crystallinity and transmittance increase, while higher temperatures $\left(>250{ }^{\circ} \mathrm{C}\right)$ result in rougher surfaces. Doping not only affects the preferred orientation but also the source compounds, solvent, and growth parameters (such as the solution concentration, feed rate, and spraying gas pressure). It is claimed that the fluorine doping using the ultrasonic spray technique $^{\mathbf{1 6 1}}$ decreases the sheet resistance from about $138 \Omega$ $\mathrm{sq}^{-1}$ to $35 \Omega \mathrm{sq}^{-1}$ and increases the optical bandgap from $3.57 \mathrm{eV}$ for a single crystal $\mathrm{SnO}_{2}$ to $3.77-3.93 \mathrm{eV}$.

Ultrasonic spray pyrolysis of FTO layers on flexible substrates leads to compact grain structures without cracks. ${ }^{162}$ Muthukumar et al. reported that an increase in the growth temperature from $360{ }^{\circ} \mathrm{C}$ to $400{ }^{\circ} \mathrm{C}$ results in an average grain size increase from $70 \mathrm{~nm}$ to $100 \mathrm{~nm}$ and an RMS roughness increase from $6.4 \mathrm{~nm}$ to $10.5 \mathrm{~nm} .{ }^{162}$ The Hall mobility increased from $11 \mathrm{~cm}^{2}$ $\mathrm{V}^{-1} \mathrm{~s}^{-1}$ to $20.1 \mathrm{~cm}^{2} \mathrm{~V}^{-1} \mathrm{~s}^{-1}$ and resistivity decreased from $1.3 \times$ $10^{-3} \Omega \mathrm{cm}$ to $6.3 \times 10^{-4} \Omega \mathrm{cm}$, with the increase of growth temperature. With the increase of film thickness from 211 to $480 \mathrm{~nm}$, there was an increase in the average grain size from $85 \mathrm{~nm}$ to $110 \mathrm{~nm}$ and the RMS roughness from $9.2 \mathrm{~nm}$ to $19.2 \mathrm{~nm}$ due to competitive grain growth processes. ${ }^{\mathbf{1 6 2}}$ Niobiumdoped $\mathrm{SnO}_{2}$ thin films of cassiterite tetragonal structure and polyhedron-shaped grains grown by spray pyrolysis are presented. ${ }^{117,163,164}$ In this deposition process, the optical transmittance increased when compared to that of undoped $\mathrm{SnO}_{2}$, while the absorption edge is red-shifted with an increase in the niobium doping concentration. ${ }^{117}$

According to Kumar et al., doping with neodymium (Nd) improves the electrical parameters of n-type $\mathrm{SnO}_{2}$ films. ${ }^{165}$ The resistivity of $\mathrm{SnO}_{2}$ films initially decreased with the $\mathrm{Nd}$ doping level up to $4 \%$ and further increased for a higher doping level of $6 \% .{ }^{165}$ The change in resistivity was found to be associated with the carrier concentration and grain boundary scattering in the doped $\mathrm{SnO}_{2}$ films. The increment in the carrier concentration and conductivity was related to the increase in $\mathrm{Nd}$ dopants that generated more carriers in the $\mathrm{SnO}_{2}$ lattice upon substitution. In a report by Serin et al., the electrical conductivity of spraydeposited polycrystalline un-doped $\mathrm{SnO}_{2}$ films was calculated using a two-point probe method as a function of substrate temperature. ${ }^{\mathbf{1 6 6}}$ The Hall mobility and electron concentration as a function of substrate temperature have been studied in detail. $^{166}$ The highest mobility of $35 \pm 1.1 \mathrm{~cm}^{2} \mathrm{~V}^{-1} \mathrm{~s}^{-1}$ was

Table 1 Properties of $\mathrm{SnO}_{2}$ thin films deposited using solution-based techniques

\begin{tabular}{|c|c|c|c|c|c|c|}
\hline Dopants & Thickness (nm) & Resistivity ( $\Omega \mathrm{cm})$ & $\begin{array}{l}\text { Transmittance } \\
(\%)\end{array}$ & Mobility $\left(\mathrm{cm}^{2} \mathrm{~V}^{-1} \mathrm{~s}^{-1}\right)$ & Year & Reference \\
\hline Undoped & 720 & $1.15 \times 10^{-3}$ & 86 & 0.61 & 2008 & Kasar et al. ${ }^{15}$ \\
\hline Sb-doped & 525 & $4.7 \times 10^{-4}$ & 60 & 11 & 2018 & Ponja et $a l^{3}$ \\
\hline P-doped & 400 & $7.2 \times 10^{-4}$ & 80 & 35 & 2018 & Powell et al. ${ }^{5}$ \\
\hline Ga-doped & 160 & 0.71 & 87.5 & $8.33 \pm 0.16$ & 2015 & Tsay et $a .^{27}$ \\
\hline Nb-doped & 550 & $9.6 \times 10^{-4}$ & 71.87 & - & 2013 & Turgut et al. ${ }^{117}$ \\
\hline Mo-doped & $1.7 \times 10^{4}$ & 1.6 & 60 & - & 2017 & Huo et al. 119 \\
\hline W-doped & $1.7 \times 10^{4}$ & 0.61 & 69 & - & 2017 & Huo et al. ${ }^{119}$ \\
\hline Mo and W co-doped & $1.35 \times 10^{4}$ & 0.35 & 56 & - & 2017 & Huo et al. ${ }^{119}$ \\
\hline F-doped & 1000 & $4.1 \times 10^{-4}$ & 75 & - & 2010 & Miao et al. ${ }^{158}$ \\
\hline F-doped & 440 & - & 84.61 & - & 2015 & Benhaoua et al. $^{161}$ \\
\hline F-doped & 211 & $8.9 \times 10^{-4}$ & 79.4 & 17.9 & 2013 & Muthukumar et al. ${ }^{162}$ \\
\hline Sb-doped & 454 & $2.81 \times 10^{-3}$ & 60.55 & 0.347 & 2013 & An et $a l^{140}$ \\
\hline Sb-doped & 340 & $1.98 \times 10^{-5}$ & 72 & - & 2013 & Lekshmy et al. ${ }^{143}$ \\
\hline Co-doped & 450 & 37.35 & 80 & - & 2010 & Bagheri et al. ${ }^{167}$ \\
\hline F-doped & 300 & $1 \times 10^{-3}$ & 80 & 28 & 2014 & Wang et al. ${ }^{168}$ \\
\hline
\end{tabular}


observed at $300{ }^{\circ} \mathrm{C}$. The mobility decreased with increasing temperature. The conductivity of $\mathrm{SnO}_{2}$ samples was found to be persistent with respect to the substrate temperature. Initially, the electrical conductivity and free-electron concentration increased with the substrate temperature and then fell laterally. However, the Hall mobility first decreased and then increased with increasing substrate temperatures. The Hall mobility values reported for $\mathrm{SnO}_{2}$ films were lower as compared to monocrystalline thin films. The low values of mobility might be due to the hindrance provided by grain boundaries with respect to carrier transport in the $\mathrm{SnO}_{2}$ polycrystalline film. The properties of doped $\mathrm{SnO}_{2}$ films prepared by solution-based techniques are tabulated in Table 1.

The solution-based approach offers a facile and an efficient process to deposit films made up of metal oxide on polyethylene terephthalate (PET) and glass substrates with low cost and scaleup opportunity. Doping of various elements shows the versatility of the solution approach towards the design of highly conducting and transparent tin oxide films. Although there are reports on the film deposition at low temperatures, achieving good crystallinity is critical to obtain desirable electrical and optical properties. Most processes require post-growth heat treatment to achieve high electrical conductivity that again limits their application in low-temperature device fabrication. Therefore, further efforts on the development of nanomaterials based on $\mathrm{SnO}_{2}$ thin films are key to resolve these existing challenges.

\subsection{Chemical vapor deposition (CVD) and metal-organic} chemical vapor deposition (MOCVD)

Wang et al. ${ }^{\mathbf{1 6 8}}$ deposited FTO films by the CVD technique with the inclusion of different additives and reported a carrier mobility of $\sim 28.5 \mathrm{~cm}^{2} \mathrm{~V}^{-1} \mathrm{~s}^{-1}$ at a high carrier concentration of $\sim 4 \times 10^{20} \mathrm{~cm}^{-3}$. The reported high mobility is associated with the development of (200) preferred orientation of the CVDgrown FTO thin films. ${ }^{168}$ In another work, Ponja et al. ${ }^{3}$ demonstrated antimony doped $\mathrm{SnO}_{2}$ thin films using aerosol assisted CVD. The samples with 4 at\% Sb dopant exhibited an electron mobility of $11.4 \mathrm{~cm}^{2} \mathrm{~V}^{-1} \mathrm{~s}^{-1}$ and a relatively high carrier density of $10^{21} \mathrm{~cm}^{-3}$ with a visible transmittance of $60 \%$. Hybrid density functional theory (DFT) calculations reveal the performance limit beyond a certain dopant level and the appearance of $\mathrm{Sb}$ (III) within the doped thin films. ${ }^{3}$ Phosphorus doped $\mathrm{SnO}_{2}$ which displays excellent electrical properties and optical properties was synthesized by using aerosol assisted CVD (AACVD). ${ }^{5}$ Phosphorus concentration plays a key role in obtaining high mobility and high visible transmittance. Both the surface morphology and crystallinity depend on the phosphorus concentration. ${ }^{5}$

Ta-doped $\mathrm{SnO}_{2}$ films were deposited on $\alpha-\mathrm{Al}_{2} \mathrm{O}_{3}$ (012) substrates by using a MOCVD method. The deposited films showed an average transmittance of $>88 \%$ in the visible wavelength range. ${ }^{169}$ When the Ta concentration was increased from 0 to 8 at $\%$, the transparency range extended to the UV-B spectral region (i.e. $280-320 \mathrm{~nm}$ ), and the optical bandgap of the films increased from 3.96 to $4.30 \mathrm{eV} \cdot{ }^{169}$ Furthermore, 4 at\% of Ta

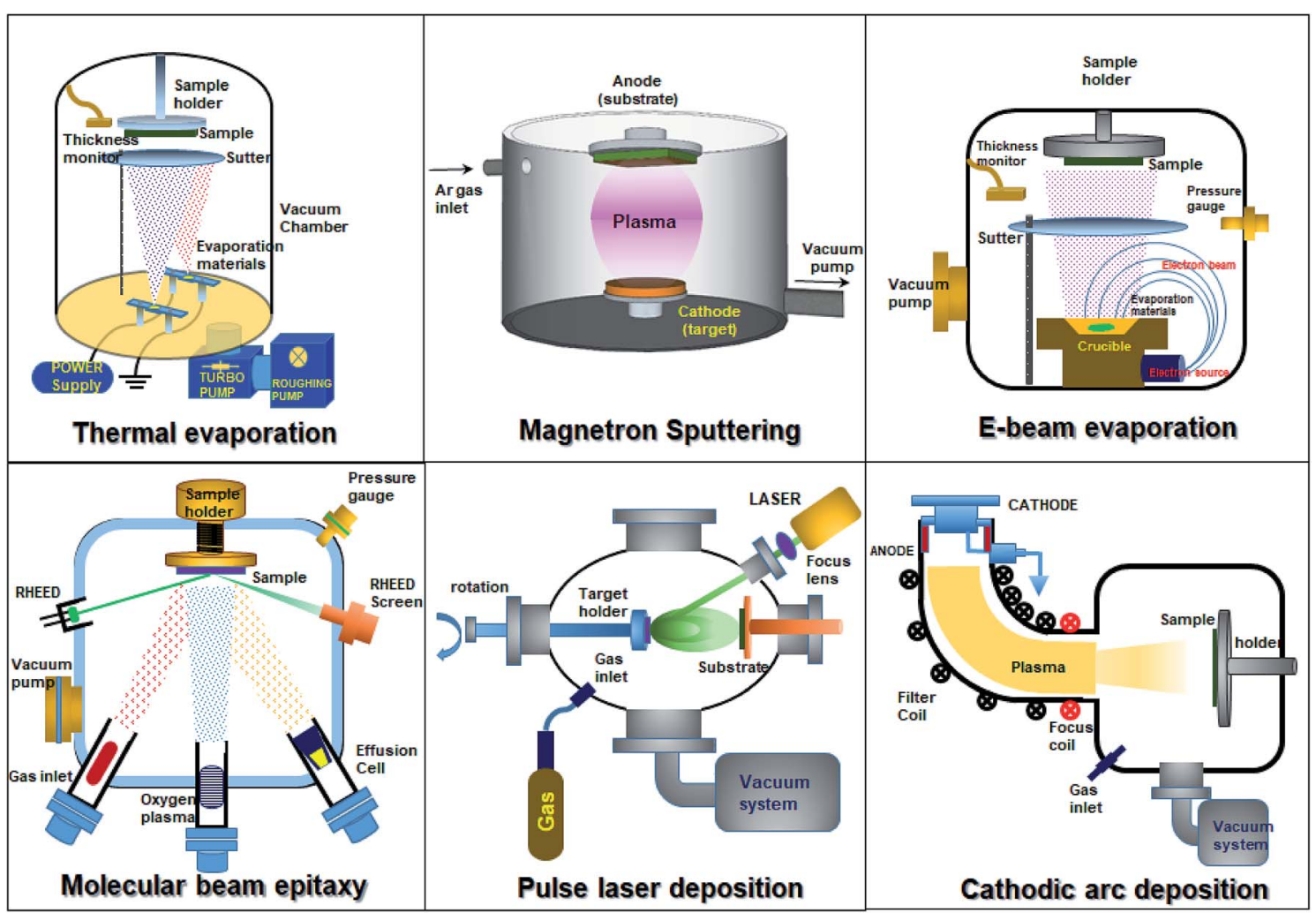

Fig. 6 Vacuum based physical vapor deposition techniques. 
doped $\mathrm{SnO}_{2}$ films showed the highest Hall mobility of $58.1 \mathrm{~cm}^{2}$ $\mathrm{V}^{-1} \mathrm{~s}^{-1}$, and the lowest resistivity of $4.0 \times 10^{-4} \Omega \mathrm{cm}$ was obtained at 6 at\% of Ta doping concentration. ${ }^{169}$ Ta-doped $\mathrm{SnO}_{2}$ epitaxial films demonstrate beneficial electrical properties and transparency extending to the UV-B light region, indicating a wide range of applications from transparent electric to photoelectric devices. ${ }^{169}$

\subsection{Doping of $\mathrm{SnO}_{2}$ through physical vapor deposition methods}

Thin-film $\mathrm{SnO}_{2}$ can be synthesized by using different physical vapor deposition techniques, namely, magnetron sputtering, pulse laser deposition, thermal evaporation, and electron beam evaporation. Fig. 6 depicts the different techniques employed to synthesize $\mathrm{SnO}_{2}$ thin films. Physical vapor deposition techniques provide high-quality thin films when compared to solution-based methods. The film thickness, composition, and electronic properties can be tuned precisely. The sputter deposition technique is commonly used for $\mathrm{SnO}_{2}$ deposition. It is a widely accepted and used technique for numerous applications including metal electrodes, transparent conductors, gas sensors, liquid crystal displays, LEDs, thin-film solar cells, and dielectric layers in low emissivity coatings for energy-saving smart windows. Due to its higher deposition rate, excellent reproducibility, competitive cost, and the possibility of using commercially available large-area sputtering systems, magnetron sputtering is a preferred technique..$^{\mathbf{2 , 2 6 , 3 1 , 1 7 0 - 1 7 5}}$ Numerous reports have been made to grow $\mathrm{SnO}_{2}$ thin films by doping using the direct sputtering technique. ${ }^{\mathbf{8 9 , 1 1 5 , 1 7 2 - 1 7 9}}$ DC magnetron sputtering offers a high deposition rate, uniformity over a largearea substrate and provides easy control over the composition of films. $\mathrm{SnO}_{2}$ films with single phase tetragonal polycrystalline structures can be deposited by sputtering and annealing in air at different temperatures. It is also worth noting that the crystallization of films grown at high substrate temperatures is enhanced. ${ }^{\mathbf{1 7 8}}$

Banyamin et al. ${ }^{89}$ demonstrated the electrical and optical properties of FTO deposited by mid-frequency pulsed DC magnetron sputtering ${ }^{89}$ from a loosely packed blended $\mathrm{SnO}_{2}$ and $F_{2}$ powder to produce homogeneous n-type thin films at low temperatures without post-deposition treatment. This method has several advantages: (1) enhanced sputtering rate without any need for reactive process control equipment, (2) low deposition temperatures, (3) suppression of arcs, and (4) the formation of dense homogeneous films. Furthermore, the loosely packed powder avoids target cracking and allows variation of the composition. ${ }^{89}$ Apart from the scattering losses at surfaces, sputter-deposited FTO has high transparency (82$85 \%$ ) in the visible region, which is independent of doping. In this process, the mean crystallite size increases with both the fluorine content and temperature, but diminishes with excess fluorine due to the solubility limit. The bandgap increases slightly with F doping (from 3.70 to $3.77 \mathrm{eV}$ ).

Polycrystalline tantalum-doped tin oxide (TTO) films can be deposited onto amorphous substrates to obtain lower resistivity using the radiofrequency (RF)-magnetron sputtering method. ${ }^{177}$
Thin film resistivity in such processes decreases exponentially from 1 to $1.7 \times 10^{-3} \Omega \mathrm{cm}$ with respect to the substrate temperature. These resulted in an average optical transmittance decrease from $97 \%$ at $600{ }^{\circ} \mathrm{C}$ to $91 \%$ at $700{ }^{\circ} \mathrm{C}$. The charge carrier density increased from $3.6 \times 10^{18} \mathrm{~cm}^{-3}$ at $300{ }^{\circ} \mathrm{C}$ to 3.3 $\times 10^{20} \mathrm{~cm}^{-3}$ at $700{ }^{\circ} \mathrm{C}$, while the Hall mobility increased from 1 to $12 \mathrm{~cm}^{2} \mathrm{~V}^{-1} \mathrm{~s}^{-1}$. With an increase in the oxygen ratio in the process gas mixture, the charge carrier density initially dropped and then increased, thereby decreasing its mobility, transmittance, and grain size. It is possible to prepare nitrogendoped tin oxide in an amorphous phase onto flexible PET substrates by RF magnetron sputtering. ${ }^{115}$ Increasing the oxygen partial pressure produces oxygen-rich smoother and more uniform films, thereby increasing the transmittance (about 80\% in the visible region) and the optical band gap (from 3.19 to $3.42 \mathrm{eV}$ for 1 to $4 \%$ partial pressure). The resistivity of nitrogendoped $\mathrm{SnO}_{2}$ is in the range of $9.1 \times 10^{-4} \Omega \mathrm{cm}$.

Low-temperature reactive DC magnetron sputtering can also be used to prepare antimony doped tin oxide films on glass and graphite substrates using a metallic tin target, without additional heat treatment. ${ }^{179}$ The electrical resistivity of such films varies with the oxygen content in the sputtering gas atmosphere as it influences the optical properties such as changes in color (yellow at $10 \%$ but brown at $16 \%$ oxygen). It also provides moderate transmission whereby at $17 \%$ oxygen, the average optical transmittance is about $74 \%$. Besides, the bandgap energy increases with the oxygen content from $2.2 \mathrm{eV}$ at below $17 \%$ to approximately $3.6 \mathrm{eV}$ at $17 \%$ of $\mathrm{SnO}_{2}$.

Dopant elements, the synthesis mechanism and postdeposition treatments play an important role in achieving high electrical conductivity and optical transparency for TCOs. ${ }^{\mathbf{1 8 0 , 1 8 1}}$ The Sb-, Ta-, Nb-, F-, arsenic (As)-, and tungsten (W)-doped $\mathrm{SnO}_{2}$ thin films have been widely explored. ${ }^{\mathbf{8 6 , 1 8 2 , 1 8 3}}$ Even though $\mathrm{Sb}$ is the frequently used dopant for $\mathrm{SnO}_{2}$ films for optoelectronic device applications, ${ }^{\mathbf{8 6 , 1 7 2}}$ the persistent issue is the strong resistivity dependency on the film thickness. ${ }^{171}$ In general, when the thickness is decreased by tens of nanometers, the resistivity of TCO thin films increased significantly. ${ }^{171}$ Shihui Yu et al. developed Sb-doped $\mathrm{SnO}_{2}$ (ATO) thin films with varying thicknesses on a glass substrate by magnetron sputtering and proposed a mechanism of varying electrical properties with respect to film thickness. ${ }^{171}$ In 2019, Bhasker Parida et al. deposited high-quality ITO films by RF magnetron sputtering with post-thermal annealing in a nitrogen environment. ${ }^{184}$ The high quality of ITO films is attributed to the combined effects of effective suppression of oxygen incorporation into films due to the post-annealing process. ${ }^{184}$

Liao et al. and Kim et al. also reported the conductive properties of $\mathrm{SnO}_{2}$ films by introducing $\mathrm{H}_{2}$ into sputtering plasma. ${ }^{185,186}$ Thin films of FTO were prepared by pulsed DC magnetron sputtering with a metal tin target through two different modes: the transition mode and the oxide mode. In the transition mode, the $\mathrm{CF}_{4}$ gas flow rate was varied, whereas, in the oxide mode, the $\mathrm{CF}_{4}$ gas flow was fixed, but the $\mathrm{H}_{2}$ gas flow was varied. ${ }^{185} \mathrm{~A}$ minimum resistivity of $1.63 \times 10^{-3} \Omega \mathrm{cm}$ with an average visible transmittance of $80.0 \%$ was obtained for the transition mode, whereas in the oxide mode the resistivity 
reduced to $8.42 \times 10^{-4} \Omega \mathrm{cm}$ with an average transmittance of $81.1 \%$. Kim et al. investigated the effect of using hydrogen plasma treatment on the structural and electronic properties of sputter-grown $\mathrm{SnO}_{2} \cdot{ }^{186}$ The electrical conductivity of the film increases due to the generation of oxygen vacancies after hydrogen plasma treatment. On the other hand, hydrogen plasma treatment etched $\mathrm{SnO}_{2}$ films and subsequently degraded their crystalline quality and optical transmittance. Zhu et al. prepared FTO films by using RF magnetron sputtering with a $\mathrm{SnO}_{2}-\mathrm{SnF}_{2}$ target in an $\mathrm{Ar}+\mathrm{H}_{2}$ atmosphere. The introduction of $\mathrm{H}_{2}$ during sputtering can improve the conductivity of FTO films. It was also revealed that the base pressure has a notable influence on the structural properties of FTO films. ${ }^{187}$ The performance of TCO is considerably affected by the crystallinity and surface morphology of the film. ${ }^{\mathbf{1 8 8 , 1 8 9}}$ Smoother surfaces reduce the contact resistance and localized field effects, ${ }^{190,191}$ whereas rougher or patterned surfaces affect the amount of light absorbed by the active layers due to entrapment of incident light (by scattering the incoming light and increasing the optical path length of light within the solar cells). ${ }^{192,193}$ A pyramidal surface is found to have a larger transmission and efficiency than those of a rectangular surface. ${ }^{188}$ The surface morphology and grain orientation of a film are affected by its own thickness. ${ }^{194}$ The electrical conductivity and transmittance of the film increases and decreases, respectively, with increasing thickness of the film. ${ }^{\mathbf{1 5 6}}$

Engineering the morphology is important for specific applications, and roughness in relation to morphology can be tuned by incorporating additives during the process. ${ }^{195}$ It is also worth noting that the resistivity depends on the crystalline orientation. ${ }^{10}$ Investigation of doped $\mathrm{SnO}_{2}$ by trivalent ions using the pulsed layer deposition (PLD) technique reveals that the films exhibit preferential orientation and have an average transmittance of $83-86 \%$. The resistivity decreases with the increase of doping from $0 \%$ to $6 \%$; however, for doping with $>6 \%$, the resistivity increases. ${ }^{\mathbf{1 9 6}}$ Fukumoto et al. demonstrated epitaxially grown high mobility Ta-doped $\mathrm{SnO}_{2}$ films on $\mathrm{TiO}_{2}$ substrates using pulsed laser deposition. ${ }^{197}$ Ta-doped $\mathrm{SnO}_{2}$ $\left(\mathrm{Sn}_{1-x} \mathrm{Ta}_{x} \mathrm{O}_{2}\right.$, TTO) thin films epitaxially grown on $\mathrm{TiO}_{2}(001)$ substrates using pulse laser deposition showed a very high Hall mobility of $130 \mathrm{~cm}^{2} \mathrm{~V}^{-1} \mathrm{~s}^{-1}$ at room temperature with a carrier density of $\sim 10^{20} \mathrm{~cm}^{-3}$ (Fig. 7). It is also worth noting that the

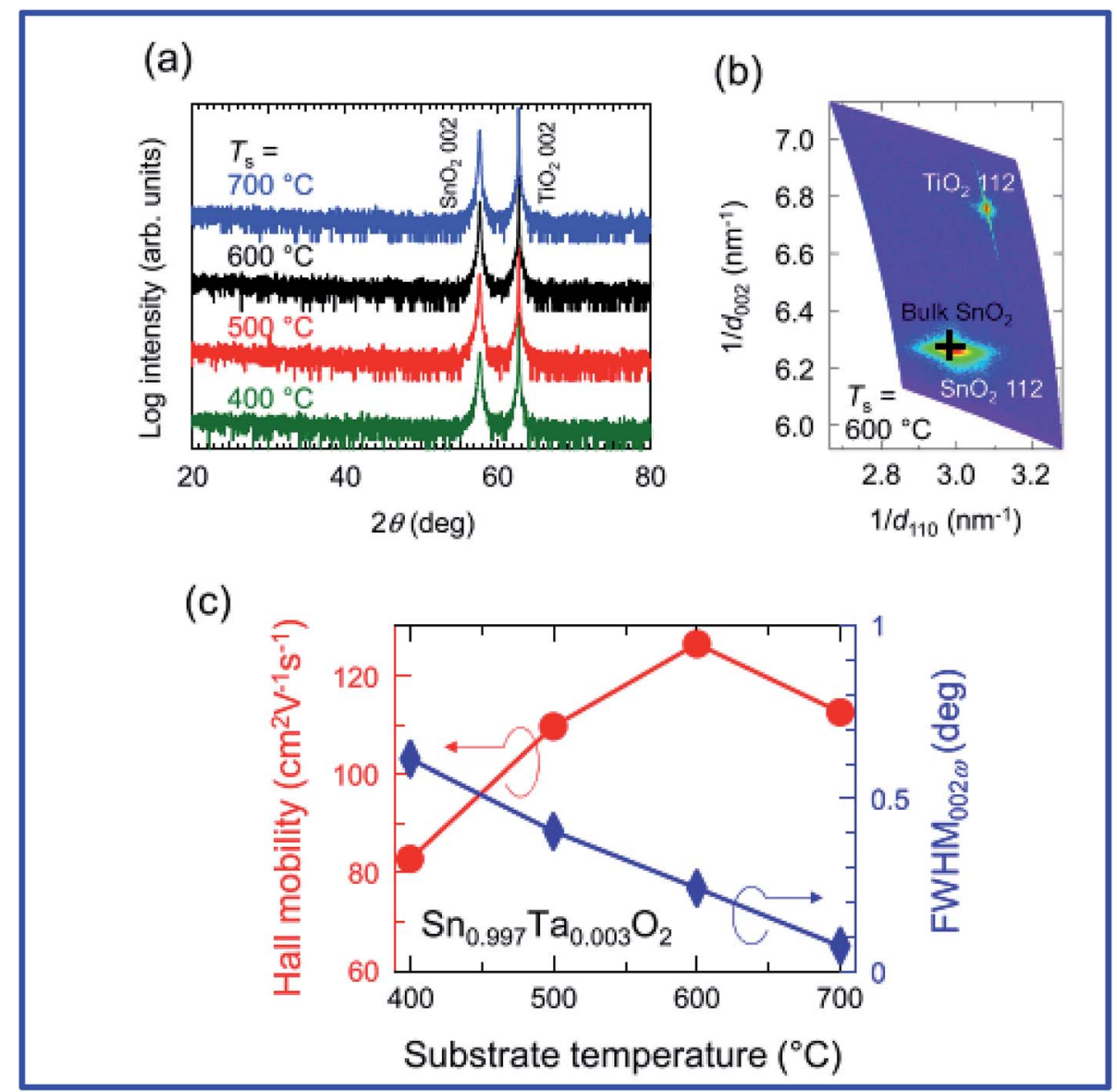

Fig. 7 (a) $\omega-2 \theta$ X-ray diffraction patterns for $\mathrm{Sn}_{1-x} \mathrm{Ta}_{x} \mathrm{O}_{2}$ (TTO) films with $x=3 \times 10^{-3}$ grown at various substrate temperatures ( $T_{\mathrm{s}}$ ). (b) $\mathrm{A}$ reciprocal space map around the asymmetric 112 diffraction peaks for a TTO film grown at $T_{\mathrm{s}}=600{ }^{\circ} \mathrm{C}$. A cross represents the peak position for bulk $\mathrm{SnO}_{2}$. (c) $T_{\mathrm{s}}$ dependence of Hall mobility ( $\mu_{\mathrm{H}}$, circles) and full width at half maximum of the rocking curve ( $\omega$ scan) for the 002 diffraction peak (FWHMO02 $\omega$, diamonds) for the $\Pi \mathrm{TO}\left(x=3 \times 10^{-3}\right)$ films, where $\mathrm{Ta}^{5+}$ ions were substituted for the $\mathrm{Sn}^{4+}$ sites and generated one electron per Ta $\left(100 \%\right.$ doping efficiency), ${ }^{197}$ presented with permission and copyright. 
Table 2 Properties of $\mathrm{SnO}_{2}$ thin films deposited using vacuum-based techniques

\begin{tabular}{|c|c|c|c|c|c|c|}
\hline $\mathrm{SnO}_{2}$ & Thickness (nm) & Resistivity $(\Omega \mathrm{cm})$ & Transmittance (\%) & Mobility $\left(\mathrm{cm}^{2} \mathrm{~V}^{-1} \mathrm{~s}^{-1}\right)$ & Year & Reference \\
\hline F-doped & 400 & $6.71 \times 10^{-3}$ & 83 & 15.1 & 2014 & Banyamin et al. ${ }^{89}$ \\
\hline AlN-doped & 200 & 0.05 & 90 & 5 & 2015 & $\mathrm{Wu}$ et al. $^{120}$ \\
\hline Al-doped & 1050 & 0.81 & 80 & 1.1 & 2010 & Entradas et al. $^{121}$ \\
\hline Al-multilayer & $\begin{array}{l}\mathrm{SnO}_{2}: 200 \\
\mathrm{Al}: 25\end{array}$ & 1.38 & $80-81$ & 0.399 & 2014 & Park et al. ${ }^{175}$ \\
\hline N-doped & 100 & $9.1 \times 10^{-4}$ & 80 & - & 2015 & Fang et al. ${ }^{115}$ \\
\hline N-doped & & $8 \times 10^{-2}$ & Above $80 \%$ & 6.75 & 2019 & Nguyen et al. ${ }^{198}$ \\
\hline Sb-doped & 220 & $1.8 \times 10^{-3}$ & & 11.6 & 2015 & Bissig et al. ${ }^{86}$ \\
\hline Sb-doped & 300 & $4.9 \times 10^{-3}$ & 74 & - & 2010 & Boltz et al. ${ }^{179}$ \\
\hline Sb-doped & 700 & $3 \times 10^{-3}$ & 80 & 6.5 & 2010 & Montero et al. ${ }^{199}$ \\
\hline Cu-multilayer & $\begin{array}{l}\mathrm{SnO}_{2}: 0 \\
\mathrm{Cu}: 14\end{array}$ & $\begin{array}{l}0.29 \\
7.79 \times 10^{-5}\end{array}$ & $\begin{array}{l}87 \\
54\end{array}$ & - & 2014 & Yu et al. ${ }^{200}$ \\
\hline Zn-doped & $850-900$ & 7.436 & 80 & 35.14 & 2012 & Ni et al. ${ }^{201}$ \\
\hline Ta-doped & & $1.7 \times 10^{-3}$ & 91 & 12 & 2014 & Weidner et $a l^{177}$ \\
\hline Ta-doped & 400 & $5.4 \times 10^{-4}$ & 85 & 25.7 & 2016 & Weidner et al. ${ }^{202}$ \\
\hline Ta-doped & $\sim 120$ & $6.0 \times 10^{-4}$ & - & 130 & 2020 & Fukumoto et al. ${ }^{197}$ \\
\hline Ta-doped & 453 & $4 \times 10^{-4}$ & 88 & 58.1 & 2019 & He et al. ${ }^{169}$ \\
\hline Cd-doped & 82 & $1.78 \times 10^{-3}$ & 84 & - & 2012 & Flores et $a .^{203}$ \\
\hline
\end{tabular}

$\mathrm{Ta}^{5+}$ ions are substituted for the $\mathrm{Sn}^{4+}$ sites and generate one electron per Ta, which suggests $100 \%$ doping efficiency for the Ta dopant. The properties of doped $\mathrm{SnO}_{2}$ films prepared by vacuum-based techniques are summarized in Table 2.

The synthesis mechanism plays an important role in $\mathrm{SnO}_{2}$ based TCOs. The surface morphology, chemical composition and bulk defects depend on the synthesis process. ${ }^{204}$ In general, the vacuum based technology provides better uniformity of the film and lower bulk defects over solution based techniques. Chemical composition can be precisely controlled using a vacuum based process. However, the solution based process provides easy synthesis, nanostructure tuning, and large scale production. Table 3 describes the advantages and challenges for different synthesis processes for $\mathrm{SnO}_{2}$.

\section{Multilayered $\mathrm{SnO}_{2} /$ metal structures for improved TCO performance}

\section{1 $\mathrm{SnO}_{2} / \mathrm{metal} / \mathrm{SnO}_{2}$ multilayers}

Multilayers of $\mathrm{SnO}_{2}$ were studied for different applications, such as the transparent conductor, low emission glass, transparent heater, etc. They typically consist of oxide-metal-oxide (OMO) trilayers. The oxide layers in such materials are composed of different oxides such as $\mathrm{TiO}_{2}, \mathrm{ZnO}, \mathrm{SnO}_{2}$, and $\mathrm{ZrO}_{2}$. Such arrangements are quite beneficial as they provide high transmittance and higher conductivity due to the presence of a metal layer. $\mathrm{SnO}_{2} /$ metal $/ \mathrm{SnO}_{2}$ multilayer structures show promising characteristics for the transparent conductor and smart coating applications. ${ }^{2} \mathrm{SnO}_{2}-\mathrm{Cu}-\mathrm{SnO}_{2}$ based multilayers are deposited onto quartz substrates using DC/RF magnetron sputtering. ${ }^{200}$ Metal-based multilayer structures have low resistivity (ranging from about $0.29 \Omega \mathrm{cm}$ for pristine to $7.79 \times 10^{-5} \Omega \mathrm{cm}$ for the $14 \mathrm{~nm}$ thin copper film), with comparable optical transmittance in the visible spectrum $(\sim 73 \%$ for $4 \mathrm{~nm}$ copper thickness, which decreases to $54 \%$ with an increase of the copper layer thickness to $14 \mathrm{~nm}$, as shown in Fig. $8 \mathrm{a}$ and b). Apart from that, the multilayer films were thinner and more durable than singlelayered TCO and single-layered metal films, respectively. The optical bandgap energies decrease from $4.27 \mathrm{eV}$ to $3.77 \mathrm{eV}$ for $14 \mathrm{~nm} \mathrm{Cu}$ thickness (Fig. 8c). With the increase of the substrate temperature from $100{ }^{\circ} \mathrm{C}$ to $300{ }^{\circ} \mathrm{C}$, the resistivity decreases and reaches its minimum value of $6.5 \times 10^{-5} \Omega \mathrm{cm}$ (Fig. 8d). A further increase in the substrate temperature leads to a drastic increase in the resistivity.

Sandwich structured $\mathrm{Zn} / \mathrm{SnO}_{2} / \mathrm{Zn}$ multilayer thin films can also be prepared on quartz glass substrates using DC and RF magnetron sputtering processes, followed by thermal treatment to obtain p-type thin films. ${ }^{201} \mathrm{SnO}_{2}: \mathrm{Zn}$ thin films exhibiting ptype behavior with a resistivity of $7.436 \Omega \mathrm{cm}$ and a carrier concentration of $2.389 \times 10^{17} \mathrm{~cm}^{-3}$ with transparency exceeding $80 \%$ were obtained at optimum annealing $\left(400{ }^{\circ} \mathrm{C}\right.$ for 6 hours). Besides these, n-type films were also obtained for the sample annealed at $300{ }^{\circ} \mathrm{C}$ for 6 hours, which might be attributed to the inability of $\mathrm{Zn}$ atoms to substitute $\mathrm{Sn}$ at lower temperatures due to the lack of activation energy. Multilayer $\mathrm{p}$ type $\mathrm{SnO}_{2} / \mathrm{Al} / \mathrm{SnO}_{2}$ thin films were prepared on a quartz substrate by using RF sputtering techniques and consequent annealing, which increased the resistivity (from $1.38 \Omega \mathrm{cm}$ at $1 \mathrm{~h}$ to $6.42 \times 10^{5} \Omega \mathrm{cm}$ at $\left.8 \mathrm{~h}\right)$ and the average transmittance $(\sim 80-$ $81 \%) .{ }^{175}$

The $\mathrm{SnO}_{2} / \mathrm{Ag}-\mathrm{Pd}-\mathrm{Cu}(\mathrm{APC}) / \mathrm{SnO}_{2}$ multilayer films for high performance flexible and transparent thin-film heaters were investigated by Kim et al. ${ }^{205}$ The $\mathrm{SnO}_{2} / \mathrm{Ag}-\mathrm{Pd}-\mathrm{Cu}(\mathrm{APC}) / \mathrm{SnO}_{2}$ multilayer films were prepared using a multi-source evaporation method..$^{205}$ In this method, the sheet resistance of $9.42 \Omega$ $\mathrm{sq}^{-1}$ and the optical transmittance of $91.14 \%$ were observed for the as-grown multilayer structure. ${ }^{205}$ In the case of multilayer formation, $\mathrm{Mn}-\mathrm{SnO}_{2}$ (MTO)/Ag/MTO thin films were prepared on a flexible polyethylene terephthalate (PET) substrate using a DC/RF sputtering system. ${ }^{206}$ The transmittance of MTO/Ag/ 
Table 3 Comparison and evolution of various technologies

Methods Advantages $\quad$ Disadvantages $\quad$ Applications

(1) Sol-gel process

(i) Low-cost and simple technique

(ii) Reproducibility

(iii) Achieves proper stoichiometry (iv) Low-temperature sintering process

(v) Functionalization

(2) Chemical bath deposition

(i) Low temperature process

(ii) Single/multiple precursor

(iii) Easy synthesis

(iv) Tunability of film structure and grain size

(v) Achieves proper stoichiometry

(vi) Strong adhesive

(vii) Large scale production

(3) Spray coating

(i) Simple, inexpensive and efficient

(ii) Several dopants with a high growth rate and reproducibility

(iii) Mass customizations

(iv) Excellent compositional homogeneity

(v) Large-area coating

(i) Easy synthesis

(ii) Can achieve a suitable thickness of the film

(iii) Excellent for laboratory scale

(iv) Quick deposition

(v) Easy integration

(5) Chemical vapor deposition

(i) Ultra-thin film

(ii) High crystal quality

(iii) Stoichiometry

(iv) Epitaxial growth

(6) Hydro/solvo thermal

(i) Simple technique

(ii) Low cost

(iii) Large scale synthesis

(7) Atomic layer deposition

(i) High-quality film

(ii) Low-temperature processing

(iii) Stoichiometric control

(iv) Excellent adhesion

(v) Ultra-thin films

(8) Thermal evaporation

(i) Synthesis of thin films over a large area with uniform thickness (ii) Low cost and reproducible film quality (i) Lack of material efficiency

(i) Synthesis of ultra-thin films $(<10$ $\mathrm{nm})$

(ii) Low wear-resistance, porosity and crystallinity

(iii) Large scale production

(iv) Easy cracking during the drying stage

(v) Presence of bulk and interface defects

(i) Long-period deposition

(ii) Synthesis of ultra-thin films $(<10$ nm)

(iii) Presence of bulk and interface defects

(iv) Various doping design and synthesis

(v) Seed layer requirement

(i) Requires high temperature

(ii) Lower conductivity

(iii) Energy consumption

(iv) Wastage of solution

(vi) Synthesis of ultra-thin film

(ii) Scalability

(iii) Wastage of material

(v) Stoichiometry

(vi) Dopant incorporation

(i) Expensive and complex process

(ii) Scalability

(iii) Wide range of doping selection

(i) Long-time reaction

(ii) Safety issues

(iii) Crystal quality

(iv) Ultra-thin film

(i) Time processing

(ii) Economic viability

(iii) Limitation of materials

(iv) Large area deposition

(i) Lower environmental stability

(ii) Mechanical durability

(iii) High temperature processing

(i) Optoelectronic devices

(ii) Thin films and coatings

(iii) Microelectronic devices

(iv) Nanostructure synthesis

(v) Catalysis for renewable energy

(vi) Renewable energy and energy storage devices

(i) Thin films and coatings

(ii) CdS layer for CIGS and CZTS based solar cells

(iii) Transparent conductors and devices

(iv) Micro-electronic devices

(v) Thin film based catalysis for renewable energy

(vi) Optical coatings

(i) Functional coatings

(ii) Energy conversion and storage

(iii) Solar cells

(iv) Highly active catalysis

(v) Supercapacitors

(vi) Hydrophobic and hydrophilic coatings

(vii) Opto-electronic devices

(i) Microelectronic semiconductor industry

(ii) Optical lenses

(iii) Photoresist-coating

(i) Microelectronic semiconductor industry

(ii) Wafer growth

(iii) High quality dielectric/insulator

(i) Large scale synthesis

(ii) Biomedical

(iii) Gas sensors

(iv) Thin/thick film

(i) Nano-coatings

(ii) Transparent conductor

(iii) Nanodevices

(iv) Catalysis and environment

(v) Energy conversion/storage

(i) Optical coating

(ii) Light-emitting diode and photovoltaic devices

(iii) Electrodes for semiconductor devices

(iv) Dielectric based multilayer for energy harvesting and saving

(9) Sputtering

(i) High-quality film

(i) High cost

(i) Optical coatings

(ii) Smart coatings

(ii) Ultra-thin layer 
Table 3 (Contd.)

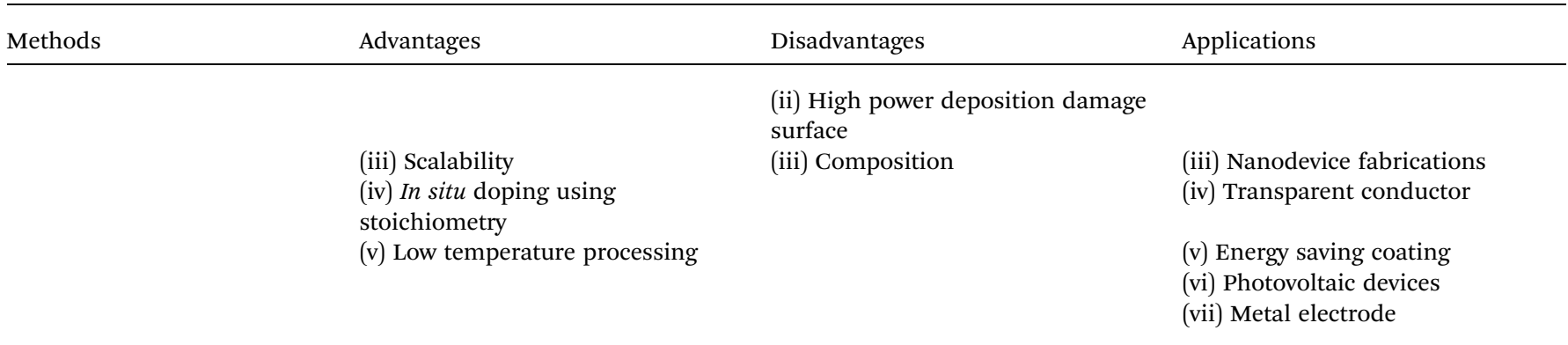

MTO multilayer films with a $550 \mathrm{~nm}$ thickness increased from $83.1 \%$ to $87.9 \%$ with an increase in the sheet resistance from 6.3 to $9.8 \Omega \mathrm{sq}^{-1}$ upon increasing the $\mathrm{O}_{2} /\left(\mathrm{Ar}+\mathrm{O}_{2}\right)$ flow rate. ${ }^{206}$ The highest figure of merit of the $\mathrm{MTO} / \mathrm{Ag} / \mathrm{MTO}$ multilayer film was $45.7 \times 10^{-3} \Omega^{-1}$ at an $\mathrm{O}_{2} /\left(\mathrm{Ar}+\mathrm{O}_{2}\right)$ flow rate of $2.8 \%{ }^{206}$ These results indicate that the $\mathrm{MTO} / \mathrm{Ag} / \mathrm{MTO}$ multilayer thin films deposited on PET substrates have high transmittance and low resistance, which make them promising materials for future flexible devices. ${ }^{206}$

On the other hand, Hwang et al. developed a three-layered $\mathrm{TiO}_{2} / \mathrm{BiVO}_{4} / \mathrm{SnO}_{2}$ (T/B/S) photo-anode which demonstrated enhanced photo-electrochemical (PEC) water oxidation performance at high visible transmittance above $510 \mathrm{~nm}$ wavelengths. ${ }^{208}$ The $\mathrm{T} / \mathrm{B} / \mathrm{S}$ photo-anode deposited by the solution spin coating method consists of three layers of sequential deposition. ${ }^{208}$ The underlying $\mathrm{SnO}_{2}$ layer creates an increased lateral grain size $(\sim 600 \mathrm{~nm})$ of the $\mathrm{BiVO}_{4}$ layer and formed a type-II heterojunction for efficient improvement in charge separation and electron transport properties. ${ }^{208}$ The $\mathrm{T} / \mathrm{B} / \mathrm{S}$ photo-anode exhibits higher photocurrent density at $1.23 \mathrm{~V}$ versus reversible hydrogen electrode $\left(\sim 2.3 \mathrm{~mA} \mathrm{~cm}^{-2}\right.$ for water oxidation and $\sim 3.7 \mathrm{~mA} \mathrm{~cm}{ }^{-2}$ for $\mathrm{H}_{2} \mathrm{O}_{2}$ oxidation). Also, it exhibits higher stability in comparison to $\mathrm{BiVO}_{4} / \mathrm{SnO}_{2}$ and pristine $\mathrm{BiVO}_{4}$ photo-anodes. ${ }^{208}$ The $\mathrm{SnO}_{2}$ based multilayer is
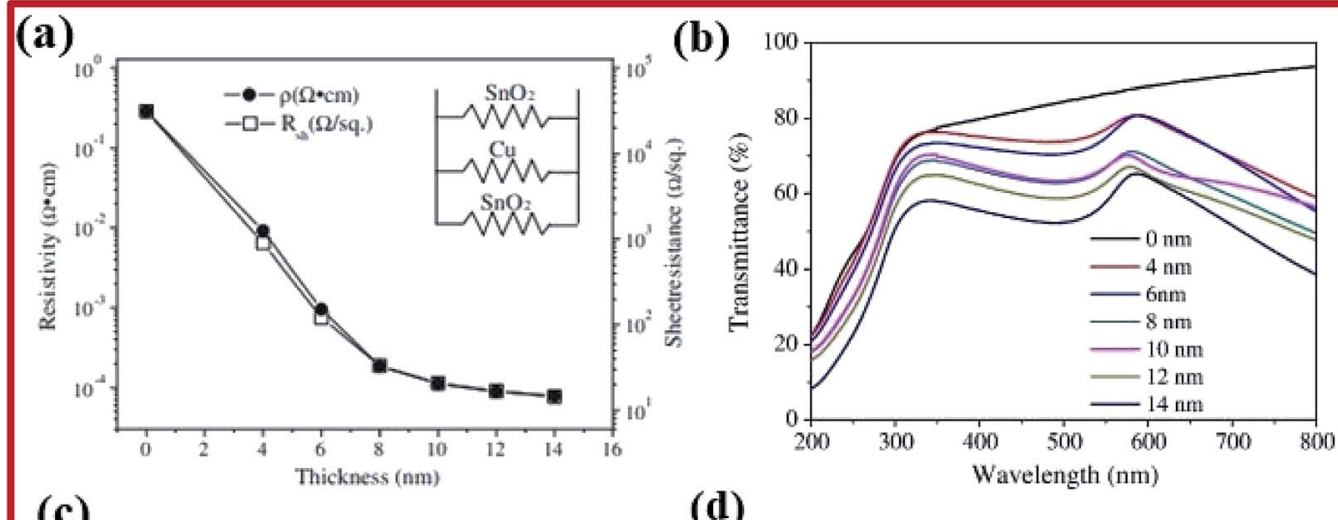

(c)

(d)
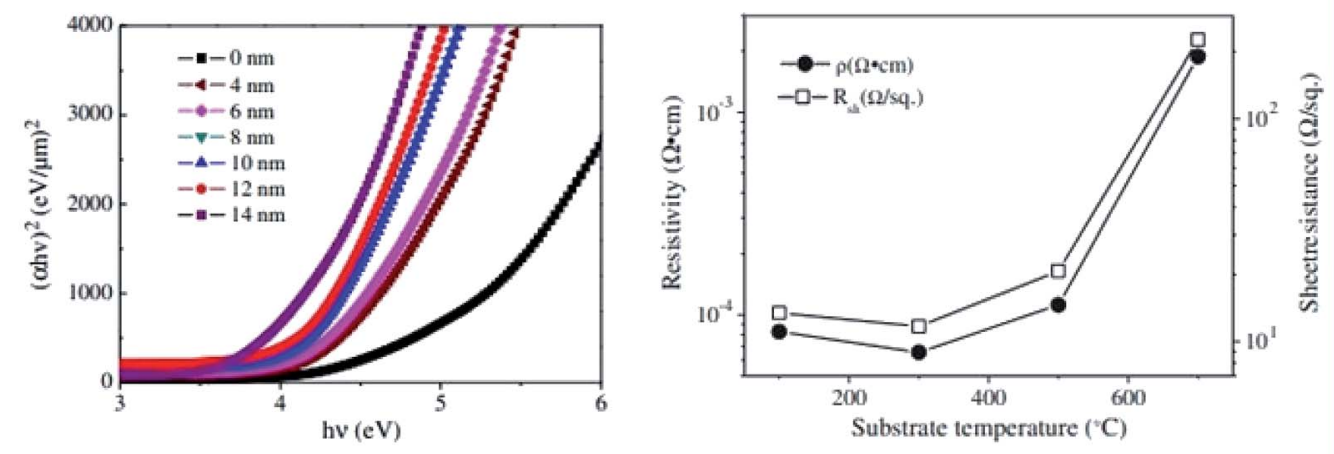

Fig. 8 (a) Resistivity and sheet resistance of $\mathrm{SnO}_{2} / \mathrm{Cu} / \mathrm{SnO}_{2}$ multilayer films deposited at $500^{\circ} \mathrm{C}$ as a function of $\mathrm{Cu}$ layer thickness. (b) Optical transmittance of $\mathrm{SnO}_{2} / \mathrm{Cu} / \mathrm{SnO}_{2}$ multilayer films deposited at $500{ }^{\circ} \mathrm{C}$. (c) The $(\alpha h \nu)^{2}$ relation for $\mathrm{SnO} / 2 / \mathrm{Cu}_{\mathrm{SnO}}$ multilayer films. (d) Sheet resistance and resistivity of $\mathrm{SnO}_{2} / \mathrm{Cu} / \mathrm{SnO}_{2}$ multilayer films developed at different substrate temperatures, ${ }^{200}$ presented with permission and copyright. 


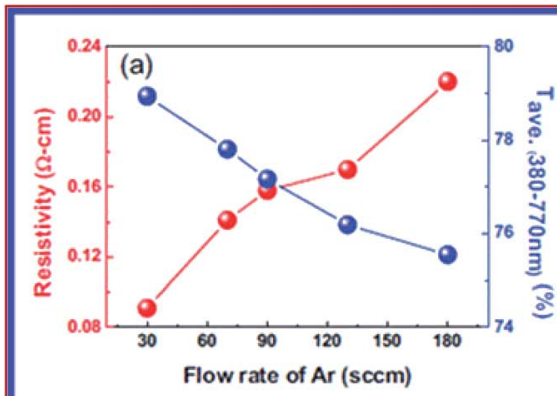

(c)
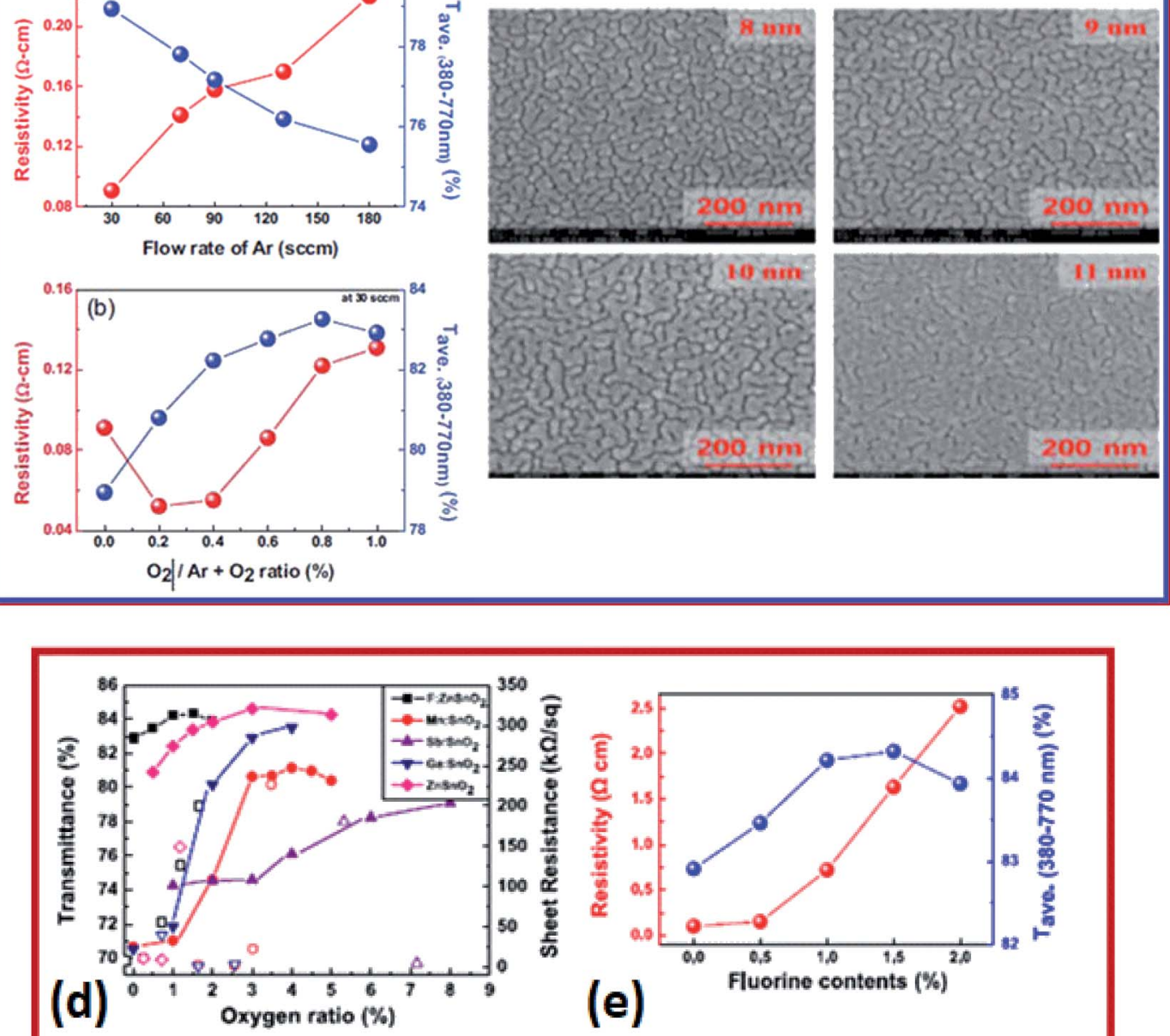

Fig. 9 (a) Resistivity of ZTO films at different argon flow rates. (b) The $\mathrm{O}_{2} / \mathrm{Ar}+\mathrm{O}_{2}$ ratio is $30 \mathrm{sccm}$. (c) Surface morphology of the silver layer on ZTO/glass with increasing thickness, ${ }^{108}$ presented with permission and copyright. (d) Average transmittance and sheet resistance of different doped tin oxide $\left(\mathrm{SnO}_{2}\right)$ thin films. (e) Resistivity and average transmittance versus fluorine content, ${ }^{207}$ presented with permission and copyright: Conductive and transparent, structured tri-layer deposited on a substrate.

a prospective technology for solar cells, solar hydrogen generation, smart coating, and flexible device applications. ${ }^{167,207,209}$ The performance of the multilayer depends on the metal layer and oxide layer. Zinc tin oxide (ZTO) shows promising characteristics for transparent conductor applications, owing to its low sheet resistance, low cost, and high transmittance. This particular set of film consists of ZTO-M-ZTO layers in which the metal $\mathrm{M}$ in the multilayer structure is $\mathrm{Ag}$. The multilayers were deposited using RF and DC magnetron sputtering (Fig. 9a and b). The electrical properties of these multilayers were studied as a function of Ar gas flow. By decreasing the Ar gas flow from 180 to $30 \mathrm{sccm}$, the resistivity of ZTO film decreased from 0.22 to $0.09 \Omega \mathrm{cm}$. However, the resistivity increased with an increase in the $\mathrm{O}_{2} / \mathrm{Ar}+\mathrm{O}_{2}$ gas flow. In such multilayers, the thickness of interlayers influences the sheet resistance. For example, the sheet resistance of ZTO film depends on the thickness of the Ag layer. The SEM images (Fig. 9c) show the morphology and thickness dependence of the Ag layer and its influence on the sheet resistance of the film. In another study, the optical transmittance and the sheet resistance of multilayer structures of various metal-doped $\mathrm{SnO}_{2}$ are shown in Fig. 9d. The resistivity of FTO is shown in Fig. 9e. As the fluorine content increases, the resistivity increases. The electrical resistivity of the FZTO film is reported to be $8 \times 10^{-5} \Omega \mathrm{cm}^{.207}$ These $\mathrm{SnO}_{2}$ based multilayers are widely used as electrode materials in organic photovoltaic (OPV) applications.

The surface morphology of metal oxides plays a crucial role in the growth of a uniform metal layer for the OMO multilayer. The performance of the OMO based TCO depends on both the metal layer and the oxide layer. The surface morphology depends on the thickness of the oxide layer (Fig. 10a-j). ${ }^{\mathbf{2 1 0}}$ Thus, it is essential to optimise the thickness of the metal oxide (e.g. $\mathrm{SnO}_{2}$ or $\mathrm{ZnO}$ ) to grow a continuous ultra-thin metal layer over the $\mathrm{SnO}_{2}$ layer. It is worth noting that the critical thickness for continuous growth of the metal layer also depends on the surface roughness of the dielectric layer (Fig. 10k and 1). ${ }^{210}$ Growth of an ultra-thin metal with a smooth surface is crucial to enhance the performance of the TCO using an OMO structure. 

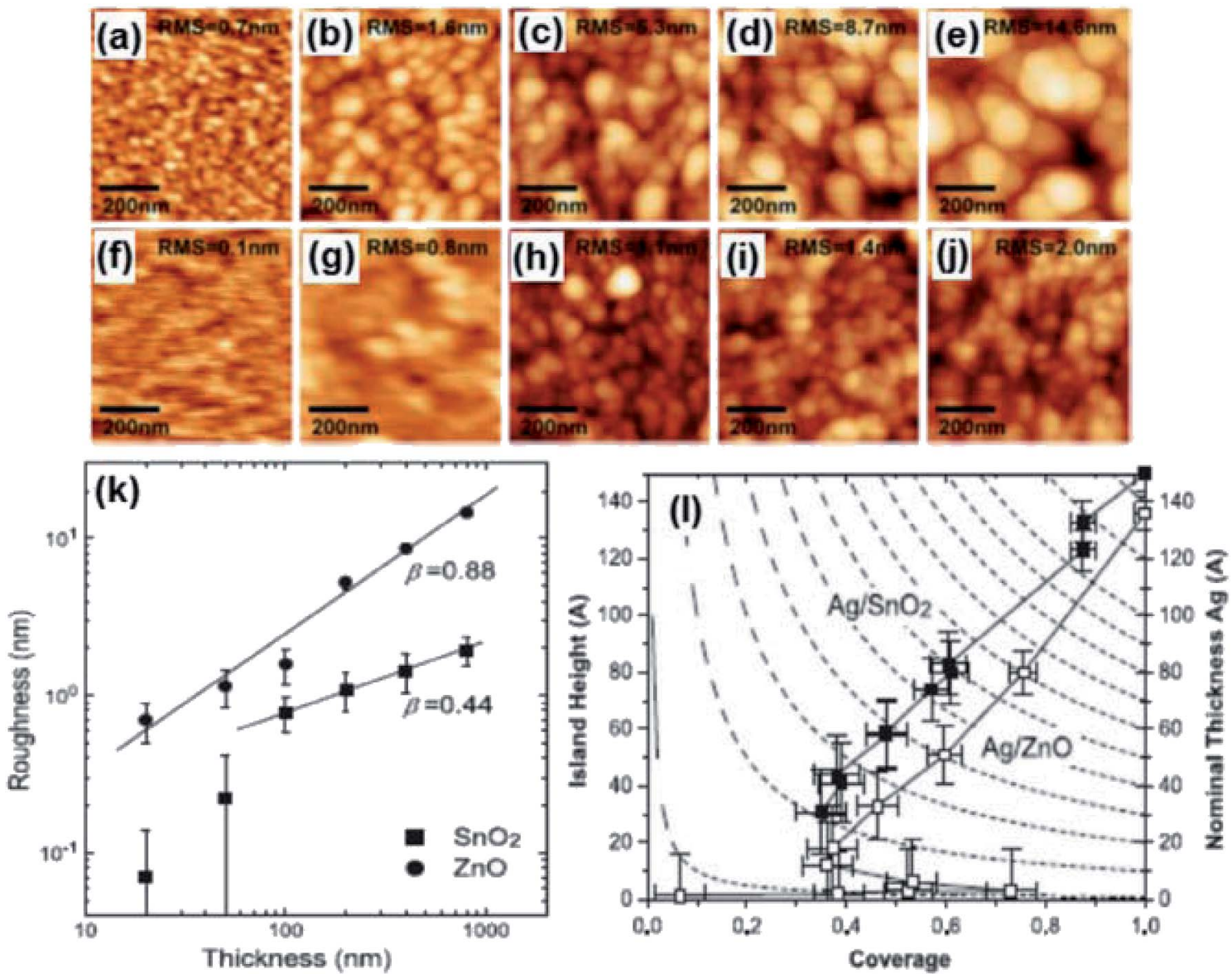

Fig. 10 AFM images of the surface of $\mathrm{ZnO}$ and $\mathrm{SnO}_{2}$ thin films of increasing thickness. $\mathrm{ZnO}$ : (a) $20 \mathrm{~nm}$; (b) $100 \mathrm{~nm}$; (c) $200 \mathrm{~nm}$; (d) $400 \mathrm{~nm}$; and (e) $800 \mathrm{~nm}$. SnO : (f) $20 \mathrm{~nm}$; (g) $100 \mathrm{~nm}$; (h) $200 \mathrm{~nm}$; (i) $400 \mathrm{~nm}$; and (j) $800 \mathrm{~nm}$. (k) Representation of the roughness versus the thin film thickness for $\mathrm{ZnO}$ and $\mathrm{SnO}_{2}$ thin films. (l) Representation of the island height against the surface coverage for successive evaporation of silver on the surface of $\mathrm{ZnO}$ and $\mathrm{SnO}_{2}$ thin films. The dotted lines represent the theoretical curves corresponding to equivalent amounts of silver. ${ }^{210}$ Reprinted from ref. 210, Copyright 2013, with permission from Elsevier.

The impact of the seeding layer on the growth of high quality thin metals can also be found in ref. 2 and 210. It is worth noting that the OMO multilayer suffers metal and oxygen diffusion during thermal treatment. Thus, the performance of the OMO based structures requires interface engineering to reduce the metal $(\mathrm{Ag})$ and oxygen diffusion into adjacent layers during high-temperature annealing. Recently, Hwang et al. demonstrated sputter-grown thermally stable $\mathrm{SnO}_{2} / \mathrm{Ag} / \mathrm{SnO}_{2}$ transparent electrodes using $\mathrm{Ni}$-doped $\mathrm{Ag}$ with improved performance due to the reduction of defects. ${ }^{211}$ Table 4 summarises the impact of a multilayer based transparent conductor on the device performances.

\subsection{Grid-structuring of the $\mathrm{SnO}_{2} /$ metal/ $/ \mathrm{SnO}_{2}$ multilayer}

Grid structuring is another promising technique that has been studied for OMO multilayer materials. ${ }^{212}$ The lift-off process is used without affecting the electrical properties of the materials. Bou et al. investigated the grid structuring of the $\mathrm{SnO}_{x}-\mathrm{Ag}-\mathrm{SnO}_{x}$

Table $4 \quad \mathrm{SnO}_{2} /$ metal based multilayer for optical devices

\begin{tabular}{|c|c|c|c|c|c|}
\hline $\mathrm{SnO}_{2} /$ metal based multilayer & Resistivity & Sheet resistance & Optical transmittance & Thickness & Reference \\
\hline $\mathrm{SnO}_{2}-\mathrm{Cu}-\mathrm{SnO}_{2}$ & $7.79 \times 10^{-5} \Omega \mathrm{cm}$ & - & $54 \%$ & $14 \mathrm{~nm}$ & Yu et al. ${ }^{200}$ \\
\hline $\mathrm{Zn} / \mathrm{SnO}_{2} / \mathrm{Zn}$ & $7.436 \Omega \mathrm{cm}$ & - & $80 \%$ & - & Ni et al. ${ }^{201}$ \\
\hline $\mathrm{SnO}_{2} / \mathrm{Ag}-\mathrm{Pd}-\mathrm{Cu}(\mathrm{APC}) / \mathrm{SnO}_{2}$ & - & $9.42 \Omega \mathrm{sq}^{-1}$ & $91.14 \%$ & - & Kim et $a l^{205}$ \\
\hline $\mathrm{Mn}-\mathrm{SnO}_{2}(\mathrm{MTO}) / \mathrm{Ag} / \mathrm{Mn}-\mathrm{SnO}_{2}(\mathrm{MTO})$ & - & $9.8 \Omega \mathrm{sq}^{-1}$ & $87.9 \%$ & $550 \mathrm{~nm}$ & Kim et al. ${ }^{206}$ \\
\hline $\mathrm{SnO}_{x}-\mathrm{Ag}-\mathrm{SnO}_{x}$ & $5.6 \times 10^{-4} \Omega \mathrm{cm}$ & $9.8 \Omega \mathrm{sq}^{-1}$ & - & - & Bou et $a l^{212}$ \\
\hline
\end{tabular}


multilayer. ${ }^{212}$ These multilayers are deposited using an e-beam evaporator. The grid structures provide low resistivity and high transmittance and are used as an anode in organic photovoltaic (OPV) devices. The surface coverage of such structures affects the properties of the multilayer thin film. The electrical measurements for the aforementioned grid tri-layered structure were carried out using an in-line four-probe method. The sheet resistance for the as-grown multilayer structure is measured to be $9.8 \Omega \mathrm{sq}^{-1}$. The resistivity of grid-based multilayers varies with the surface coverage. The resistivity increases with decreased coverage from a value of $2.9 \times 10^{-4} \Omega \mathrm{cm}$ for the full tri-layer to $5.6 \times 10^{-4} \Omega \mathrm{cm}$ for the most transparent multilayer. ${ }^{212}$

The Mn-doped $\mathrm{SnO}_{2}$ (MTO) multilayer structure is proven to be an efficient system (Fig. 11). ${ }^{209}$ The MTO/Ag/MTO multilayer electrode was deposited onto a patterned glass substrate by RF sputtering at room temperature. The electrical resistivity in a binary layered structure Ag/MTO/glass depends on the thickness of Ag. ${ }^{108,209}$ As the thickness of $\mathrm{Ag}$ is increased, the resistivity decreases. This decrease in resistivity may be attributed to the increase in the carrier concentration and hence the mobility (Fig. 11a). According to this study, the sheet resistance of the MTO/Ag/MTO layered structure was reported to be $10.1 \Omega \mathrm{sq}^{-1}$ for the $40 \mathrm{~nm}$ thick MTO film. This result is similar to that of commercial ITO. Due to these properties, MTO based heterostructures were applied as electrode materials for solar cells. The current density of the MTO/Ag/MTO multilayer electrode is compared with the commercial ITO, as shown in Fig. 11c. It is reported that the OPV parameters for MTO $(40 \mathrm{~nm}) / \mathrm{Ag}(11 \mathrm{~nm}) /$ MTO $(40 \mathrm{~nm})$ multilayers are comparable to the commercial ITO electrode. Yu et al. demonstrated a high value of the figure of merit $\sim 7.8 \times 10^{-2} \Omega^{-1}$ for FTO $(20 \mathrm{~nm}) / \mathrm{Ag}(7 \mathrm{~nm}) / \mathrm{FTO}(30$ $\mathrm{nm})$ multilayers, whereas the average optical transmittance is $95.5 \%$ in the visible range of wavelengths at a resistivity of $8.8 \times$ $10^{-5} \Omega \mathrm{cm} .^{11}$

\section{Impact of $\mathrm{SnO}_{2}$ based TCO and ETL on OPV and OLED devices}

\subsection{Impact of $\mathrm{SnO}_{2}$ on OLED devices}

A typical OLED consists of an anode deposited on a glass substrate, one or more charge transport and light emitting layers and a low work function cathode. Under an external bias, the holes and electrons are injected from the anode and the cathode, respectively, through a transport layer into the emitting material as the recombination generates excitons. These excitons activate the light emitting material to emit photons (light) because of the radiative recombination. Transparent materials are more favorable for these OLED devices. In particular, $\mathrm{SnO}_{2}$ as an n-type TCO is a good choice of material for different components in OLEDs.

Indium-free $\mathrm{SnO}_{2}$ may also be a promising electrode material because of its high transparency and large bandgap. More importantly, the fabrication of $\mathrm{SnO}_{2}$ is compatible with many

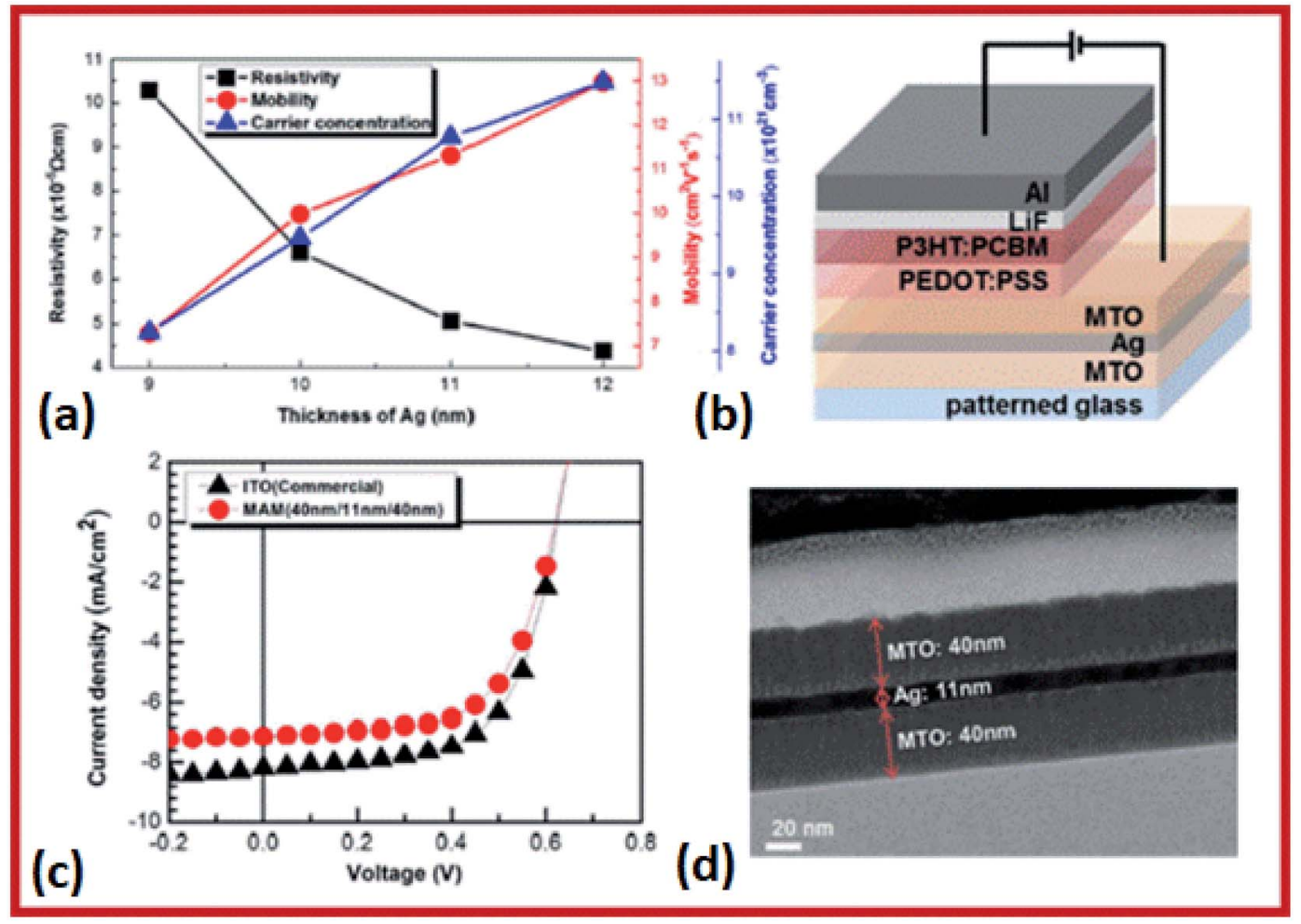

Fig. 11 (a) Electrical properties of MTO/Ag/MTO films. (b) Schematic of conventional BHJ-OPV having the MTO/Ag/MTO multilayer electrode. (c) Current-voltage characteristics of the optimized MTO/Ag/MTO multilayer and ITO as reference based on OPVs. (d) TEM (cross-section) for the MTO/Ag/MTO multilayer electrode, ${ }^{209}$ reproduced with permission and copyright. 


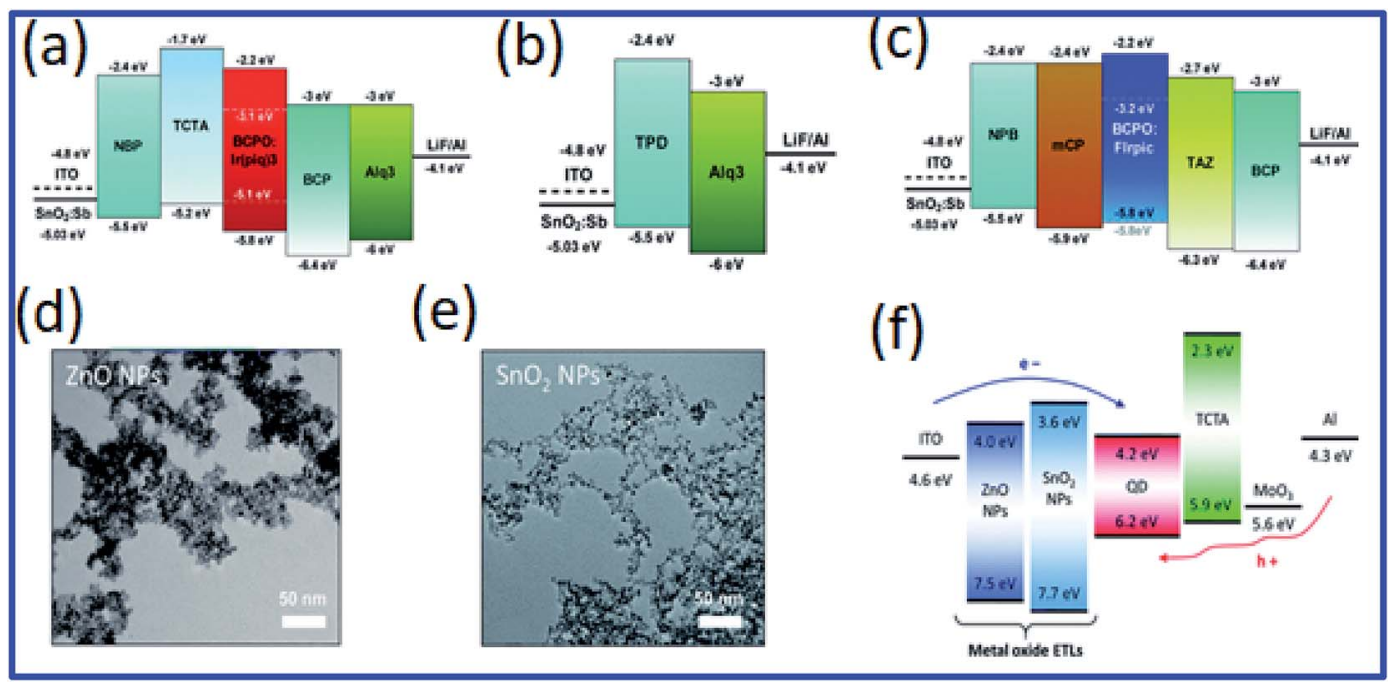

Fig. 12 Energy band diagrams of (a) red, (b) green, and (c) blue OLEDs with $\mathrm{SnO}_{2}$ :Sb or ITO as the anode electrode. Reproduced with permission. ${ }^{97}$ Copyright: The Royal Society of Chemistry 2016. The TEM images of (d) $\mathrm{ZnO}$ or (e) $\mathrm{SnO}_{2} \mathrm{NPs}$; (f) energy level diagram of QD-LEDs with $\mathrm{ZnO}$ or $\mathrm{SnO}_{2}$ NPs as the ETLs. Reproduced with permission. ${ }^{213}$ Copyright: The Royal Society of Chemistry 2020.

techniques. M. Esro et al. ${ }^{97}$ demonstrated that the Sb-doped $\mathrm{SnO}_{2}\left(\mathrm{SnO}_{2}: \mathrm{Sb}\right)$ deposited through a facile low-cost spray pyrolysis technique is a promising anode material for largescale and flexible OLEDs. By varying the $\mathrm{Sb}$ concentrations, at $2 \mathrm{wt} \%$, the $\mathrm{SnO}_{2}: \mathrm{Sb}$ film exhibits the lowest sheet resistivity and the highest electron mobility. The $\mathrm{SnO}_{2}: \mathrm{Sb}(2 \mathrm{wt} \%)$ shows a similar surface roughness in comparison with the commercially available ITO anode. Regarding the optical properties, such as making of red, green, and blue OLED devices, the energy level alignment comparison shown in Fig. 12a-c indicates that the $\mathrm{SnO}_{2}: \mathrm{Sb}$ anode has a lower work function than that of ITO, which is favorable for the hole injection. The three different-color OLED devices deposited on the $\mathrm{SnO}_{2}$ :Sb anode show comparable electrical and optical performances with those deposited on ITO. These results clearly indicate the great potential of the $\mathrm{Sb}$-doped $\mathrm{SnO}_{2}$ electrode in flexible and largescale OLEDs fabrication.

Currently, $\mathrm{SnO}_{2}$ is one of the best electron transporting materials (ETMs) in perovskite solar cells because of its excellent electron mobility; therefore, it is being introduced into other related devices as an ETL. Park et al. ${ }^{213}$ investigated colloidal quantum dot LEDs (QD LEDs) because of their tunable emissive light with pure color and very high photoluminescence quantum yield (almost reaching $100 \%$ ). In the QD LEDs, ZnO is the widely used ETM; however, it often allows more electrons to be transported to the QD emissive layer, causing a hole-electron imbalance. This is also the main barrier for high-performance and stable QD LEDs. Park et al. ${ }^{213}$ compared the properties of $\mathrm{SnO}_{2}$ as the ETM with those of ZnO. Transmission electron microscopy (TEM) and atomic force microscopy (AFM) images indicate that $\mathrm{SnO}_{2}$ NPs have a similar size (Fig. 12d and e) to that of ZnO NPs but a smoother surface, which allows better layer-by-layer connection. Both $\mathrm{SnO}_{2}$ and $\mathrm{ZnO} \mathrm{NPs}$ have a similar electron mobility in OFETs; however, the carrier concentration of $\mathrm{ZnO}$ is more than two times higher than that of
$\mathrm{SnO}_{2}$, which will cause excessive electron injection, leading to imbalance with holes. $\mathrm{SnO}_{2} \mathrm{NPs}$, however, exhibit lower carrier concentration but the same electron mobility, indicating the excellent transporting ability to balance the holes to result in improved device performance. Although the $\mathrm{LUMO}$ of $\mathrm{SnO}_{2} \mathrm{NPs}$ is slightly higher than that of ZnO NPs (Fig. 12f), the overall QD LED device performance with $\mathrm{SnO}_{2} \mathrm{NPs}$ is higher than that of the device with ZnO NPs, including a lower turn-on voltage, higher maximum luminance, improved EQE roll-off property and better power efficiency.

Another example about $\mathrm{SnO}_{2}$ as the ETM is reported by Hong et $a .^{214}$ where they studied the difference in the OLED device performance caused by the relative position of $\mathrm{SnO}_{2}$ and the organic emitter (copper phthalocyanine, CuPc). Two devices were fabricated with different layer-by-layer structures, as shown in Fig. 13a and d. In the normal device, CuPc is deposited on top of $\mathrm{SnO}_{2}$ (CuPc-on-SnO ${ }_{2}$, Fig. 13e), while in the inverted device, the $\mathrm{SnO}_{2}$ is deposited on top of $\mathrm{CuPc}\left(\mathrm{SnO}_{2}\right.$-onCuPc, Fig. 13b). Because of the much higher melting temperature of $\mathrm{SnO}_{2}$, the hot $\mathrm{Sn}$ atoms during deposition on top of $\mathrm{CuPc}$ will cause a breakdown of the weak bonds that existed in $\mathrm{CuPc}$, resulting in a chemical reaction to form CuPc-Sn. Therefore, its charge distribution is changed compared with that of the pristine CuPc, which results in the increment in both the interface dipole and hole injection barrier (Fig. 13c). If the $\mathrm{SnO}_{2}$ is first deposited, the low processing temperature of CuPc will not provide sufficient energy for the formation of the condensation reaction (CuPc-Sn). In this case, it avoids those negative effects that occurred in the $\mathrm{SnO}_{2}$-on-CuPc structure (Fig. 13f). The device performance with CuPc-on- $-\mathrm{SnO}_{2}$ is found to be better in terms of both the operating voltage and the luminance than that of the device with the $\mathrm{SnO}_{2}$-on-CuPc structure.

For many organic devices, interface modification is an effective way to improve the device performance. Taking that into consideration, Lee et $a l .{ }^{215}$ applied $\mathrm{SnO}_{2} \mathrm{NPs}$ as an interface 


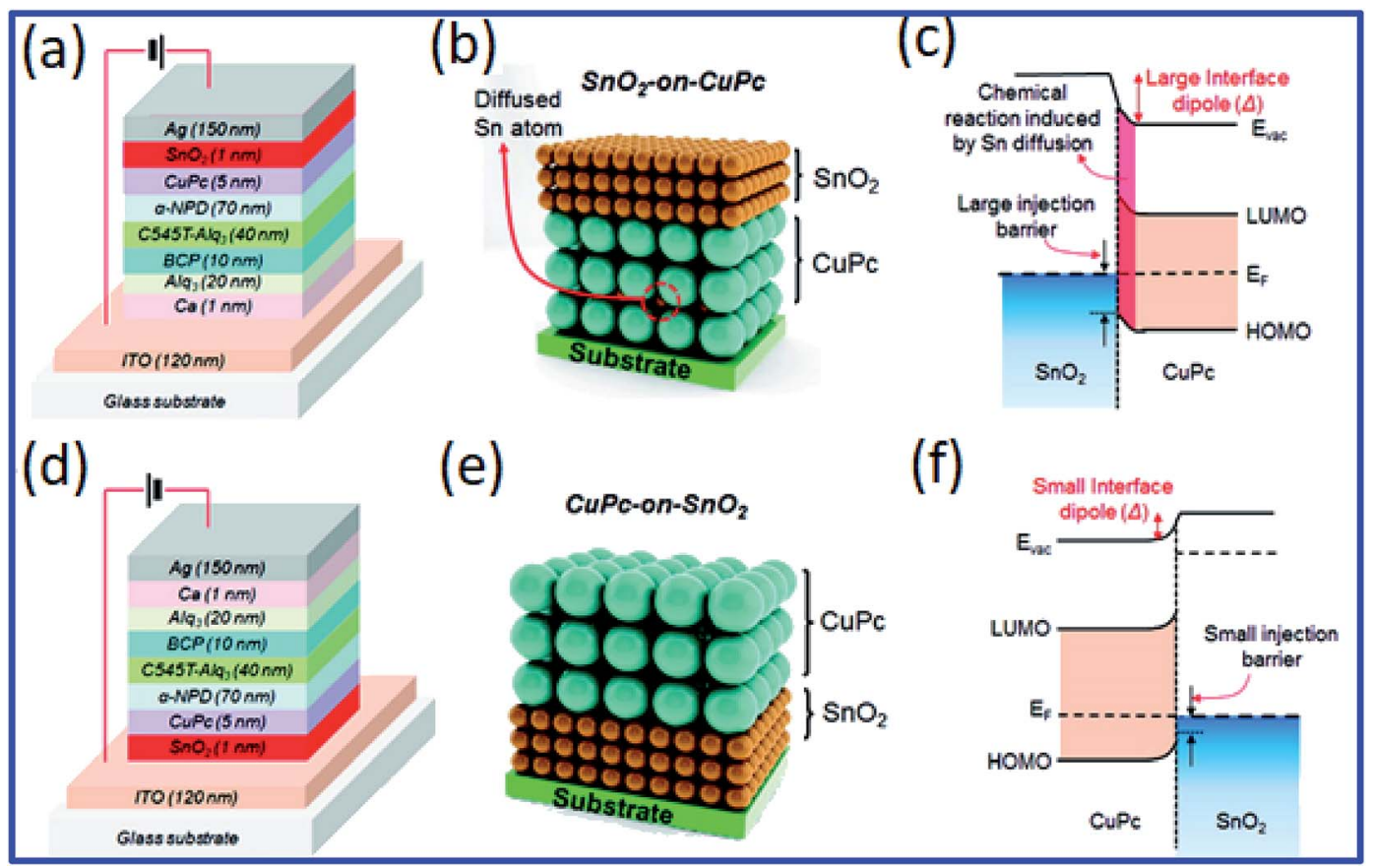

Fig. 13 Device structures of (a) normal OLEDs and (d) inverted OLEDs. (b and e) Schematic diagram and (c and f) energy band structure of the (b and c) diffused $\mathrm{SnO}_{2}$-on-CuPc and (e and f) abrupt CuPc-on- $\mathrm{SnO}_{2}$ interface. Reproduced with permission. ${ }^{214}$ Copyright: American Chemical Society 2011.

layer between the ITO cathode and the ETL in an inverted bottom-emission (ITO direction) OLED device. The work function of $\mathrm{SnO}_{2}$ nanoparticles was found to be between the ITO and the ETL. That was helpful to form a ladder-like energy alignment from ITO to the emitter to facilitate the electron injection. Additionally, the $\mathrm{SnO}_{2}$ film morphology spin-coated from $3 \mathrm{wt} \%$ aqueous solution was found to be the smoothest, which is favorable for establishing contact with the ETL and the electron transport, delivering more electrons to the emitter layer to balance the holes injected from the anode. The performance measurement of the OLED device indicates that the operating voltage to achieve $1000 \mathrm{~cd} \mathrm{~m}^{-2}$ luminance decreased significantly from $24.0 \mathrm{~V}$ (without $\mathrm{SnO}_{2}$ ) to $\sim 17.0 \mathrm{~V}$ (with $\mathrm{SnO}_{2}$ ) with an enhanced $\mathrm{EQE}$ from $8.2 \%$ to $15.6 \%$.

These samples clearly indicate that, to get the best performance, the properties of $\mathrm{SnO}_{2}$ should be carefully considered; for example, the work function, film morphology, and processing method. Successful cases are given above by applying $\mathrm{SnO}_{2}$ in different layers of the device to fulfill specific roles. Therefore, in future research, to better incorporate $\mathrm{SnO}_{2}$ in OLEDs, the relationship between $\mathrm{SnO}_{2}$ and the neighbour layers should be carefully studied to realize optimized charge carrier transport.

\subsection{Impact of $\mathrm{SnO}_{2}$ on $\mathrm{OPV}$ devices}

The OPV device has two types of generic structures: a conventional structure and an inverted structure. The basic geometry of the conventional device has continuous layers of ITO (anode)/PEDOT:PSS/active layer/metal (cathode). Such a structure is unfavorable for device stability which is one of the three most crucial factors for OPV commercialization. The other two are the power conversion efficiency (PCE) and scalability. Therefore, an inverted device geometry with a general structure of ITO (cathode)/metal oxide/active layer/ metal (anode) is developed in which metal oxide is used as the electron transporting layer deposited on top of the ITO cathode. Compared with the conventional device where PEDOT:PSS is deposited on top of the ITO anode, ${ }^{216-222}$ the stability of the inverted device is improved since the metal oxide will not erode the ITO as the acidic PEDOT:PSS (pH: 1-2) does. Besides, the metal oxide will also protect the active layer from oxygen and humidity. ${ }^{223,224}$ Moreover, the cathode in the conventional devices is generally of low-work-function metals, such as aluminium (Al) and calcium (Ca). These metallic cathode materials are easy to oxidize and even further hydrolyze in an ambient atmosphere. In the inverted device, however, the metals used as an anode are high-work-function materials, such as $\mathrm{Ag}$ and $\mathrm{Au}$, which have higher work functions than those of $\mathrm{Al}$ and $\mathrm{Ca}$. Because of this concern, the device engineering is mostly focused on inverted devices. Although the active layer materials play significant roles in improving the device performance, there are also many possibilities in judicially selecting suitable materials in the metal oxide layer. Currently, the widely used metal oxides in the ETL are $\mathrm{TiO}_{2}$ and $\mathrm{ZnO}$. That is mainly because of their high transparency and suitable work function. However, there are some disadvantages, such as the low electron mobility of $\mathrm{TiO}_{2}$, the instability of both $\mathrm{TiO}_{2}$ and $\mathrm{ZnO}$ and the hightemperature processing conditions of $\mathrm{TiO}_{2}$. All these motivate the development of alternative materials for the electron transporting layer. $\mathrm{SnO}_{2}$ is one of the best electron transporting materials in perovskite solar cells because of its high 
transparency, ultrahigh electron mobility $\left(>200 \mathrm{~cm}^{2} \mathrm{~V}^{-1} \mathrm{~s}^{-1}\right)$, non-light-soaking problems, and high chemical, photostability and hole blocking properties. ${ }^{25}$ Despite these favorable features for efficient electron transport, the application of $\mathrm{SnO}_{2}$ in OPVs is rare. One reason is that the trap-assisted recombination at the $\mathrm{SnO}_{2}$ surface is severe and requires surface modification to enable its compatibility with the organic blends in the active layer of OPV devices.

An example of the comparison of $\mathrm{SnO}_{x}$ with $\mathrm{TiO}_{x}$ and $\mathrm{ZnO}$ as the electron extraction/transporting material was reported by Trost et al. ${ }^{226,227}$ One of the advantages of $\mathrm{SnO}_{x}$ as the ETM is demonstrated, i.e., the light-soaking issue faced by $\mathrm{TiO}_{x}$ and $\mathrm{ZnO}$ is not a problem in $\mathrm{SnO}_{x}$ ETM-based OPV devices. Light soaking is defined as the activation of $\mathrm{TiO}_{x}$ and $\mathrm{ZnO}$ by UV light to make the resultant devices show good performance. However, the activation will take some time to finish, and in the meantime, the organic donor materials in the active layer may degrade under UV light illumination. Therefore, UV light protection is very much necessary for OPV devices. As shown in Fig. 14a and b, without UV light activation (filtered AM 1.5), a sigmoidal $J-V$ curve is observed in $\mathrm{TiO}_{x}$ ETM-based inverted OPV devices with an inferior efficiency of $0.4 \%$. However, the $\mathrm{SnO}_{x}$ ETM-based device shows a largely enhanced efficiency of $5.7 \%$. Although the exact reason for the light-soaking issue has not been clarified, it is an acceptable concept that the UV light activation induces oxygen desorption on the $\mathrm{TiO}_{x} / \mathrm{ZnO}$ surface that causes the decrement of work function for better electron extraction/transport. For the $\mathrm{SnO}_{x}$ film, UV light illumination does not change its work function much. This, combined with its suitable value, allows better performance to be achieved for $\mathrm{SnO}_{x}$ ETM-based inverted OPVs.
Although the light-soaking issue is resolved in $\mathrm{SnO}_{x}$ ETMbased inverted OPVs, as mentioned above, the charge traps and charge recombination exist on the $\mathrm{SnO}_{x}$ surface. Therefore, surface modification is required to avoid these traps and improve the performance of the inverted OPVs. Geng et al. ${ }^{228}$ investigated the preparation of a $\mathrm{SnO}_{2}$ and poly(3hexylthiophene) (P3HT) composite. Although this composite is not used in OPV devices, this study confirmed that the $\mathrm{SnO}_{2}$ surface modification generates favorable properties in charge carrier transport. On the surface of $\mathrm{SnO}_{2}$ nanoparticles, highly crystalline domains are favorable for charge carrier transport. However, the porous network restricts further improvement in mobility. The semicrystalline P3HT is used as a filler in the porous structures of $\mathrm{SnO}_{2}$ nanoparticles, forming a $\mathrm{P} 3 \mathrm{HT}-\mathrm{SnO}_{2}$ composite, as shown in Fig. 14c. After thermal annealing, the electron transfer inside the composite is promoted because of two reasons. First, the chemical bonds formed between $\mathrm{SnO}_{2}$ and P3HT, combined with the highly crystalline feature of $\mathrm{P} 3 \mathrm{HT}$, is responsible for the electron transfer from P3HT to $\mathrm{SnO}_{2}$ (Fig. 14d and e), resulting in more free charges in $\mathrm{SnO}_{2}$ nanoparticles. Second, thermal treatment in a high-pressure oxygen atmosphere for $\mathrm{SnO}_{2}$ allows for elimination of oxygen vacancies to increase the crystallinity of $\mathrm{SnO}_{2}$. The synergistic effects of more free charges and high crystallinity of the $\mathrm{SnO}_{2}$ section in the $\mathrm{P} 3 \mathrm{HT}-\mathrm{SnO}_{2}$ composite contribute to the higher electron mobility than those of pristine $\mathrm{P} 3 \mathrm{HT}$ or $\mathrm{SnO}_{2}$ film. This work elegantly demonstrates the feasibility of surface modification of $\mathrm{SnO}_{2}$ to promote its application as an electron transporting material in inverted OPVs.

Shen et al. ${ }^{64}$ reported that a (poly-[(9,9-bis(3-( $N, N$-dimethylamino)propyl)-2,7-fluorene)-alt-2,7-(9,9-dioctylfluorene)]), PFN,

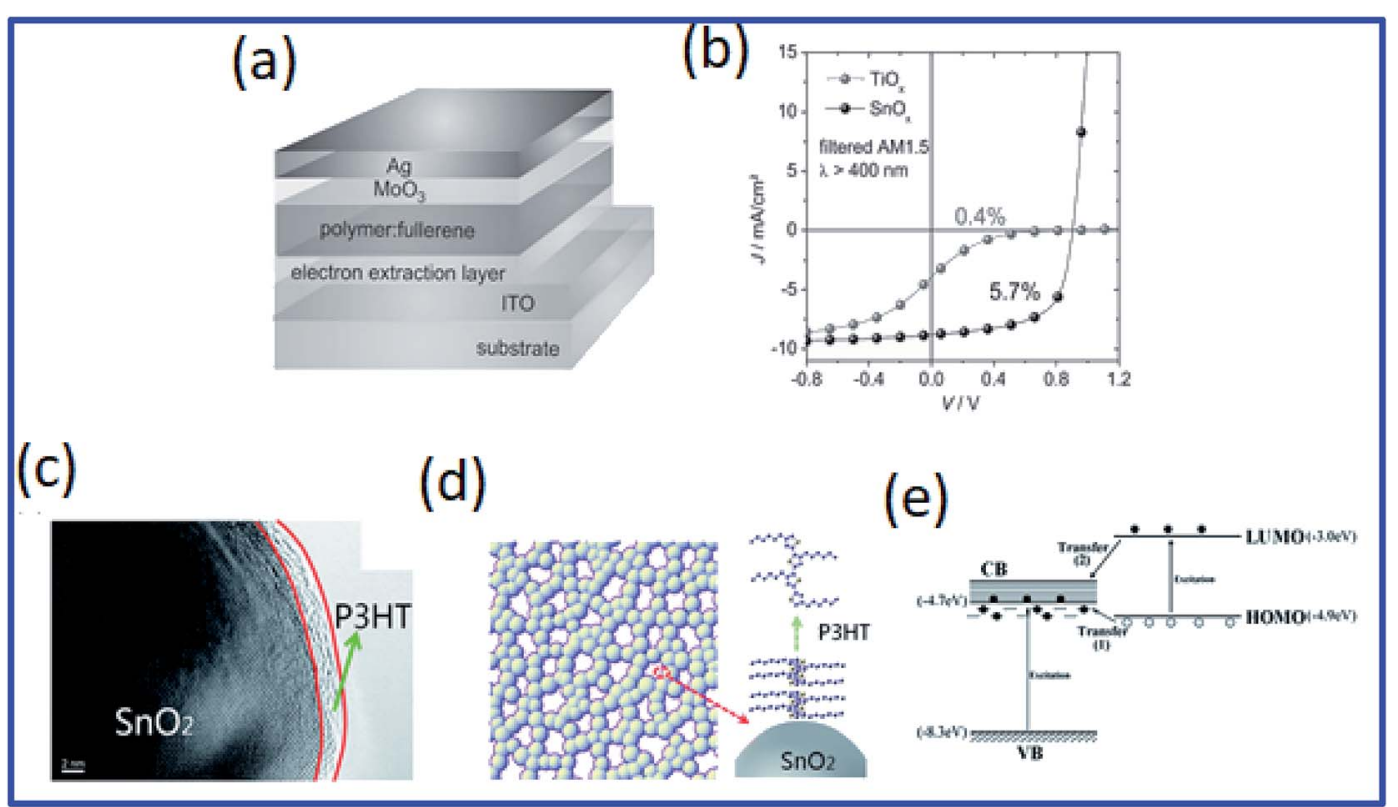

Fig. 14 (a) Layer sequence of the inverted OPV device with $\mathrm{TiO}_{x}$ or $\mathrm{SnO}_{x}$ as electron extraction layers and (b) J-V characteristics of the corresponding cells under AM 1.5G illumination with a UV blocking filter $(\lambda>400 \mathrm{~nm})$. Active layer: PCDTBT:PC ${ }_{71} B M$. Reproduced with permission. 226 Copyright: WILEY-VCH Verlag GmbH \& Co. KGaA, Weinheim 2015. (c) HRTEM image of a $\mathrm{SnO}_{2}$ nanocrystal covered with a P3HT thin layer. (d) Schematic structure of the $\mathrm{P} 3 \mathrm{HT}-\mathrm{SnO}_{2}$ composite semiconductor. (e) Energy level alignment of $\mathrm{P} 3 \mathrm{HT}$ and $\mathrm{SnO}_{2}$. Reproduced with permission. ${ }^{228}$ Copyright: The Royal Society of Chemistry 2016. 


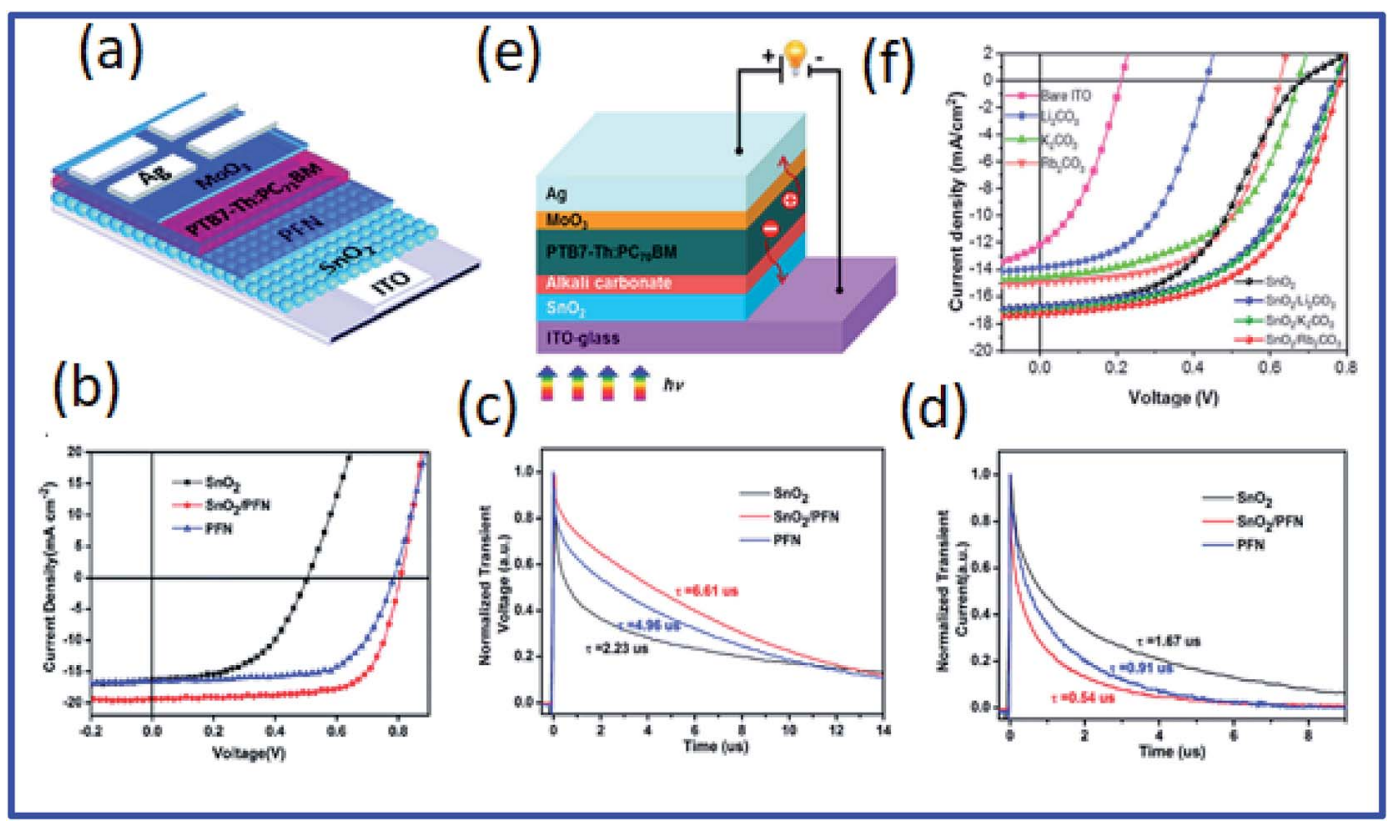

Fig. 15 (a) Device structure of the inverted OPVs by integrating low temperature solution-processed $\mathrm{SnO}_{2}$ nanocrystals and a PFN stacked structure as an ETL. (b) J-V characteristics of $\mathrm{SnO}_{2}$-only, $\mathrm{SnO}_{2} / \mathrm{PFN}$, and PFN-only devices tested under AM $1.5 \mathrm{G}$ in ambient air. (c) Transient photovoltage and (d) transient photocurrent decay curves based on $\mathrm{SnO}_{2}$ and $\mathrm{SnO}_{2} / \mathrm{PFN}$ and PFN ETLs. Reproduced with permission. ${ }^{64}$ Copyright: The Royal Society of Chemistry 2018. (e) Device structure of the inverted OPVs using $\mathrm{SnO}_{2}$ Or SnO$_{2} /$ alkali carbonates as ETLs and (f) the J-V characteristics under standard AM 1.5G. Reproduced with permission. ${ }^{225}$ Copyright: American Chemical Society 2018.

layer deposited on the $\mathrm{SnO}_{2}$ film can adjust the energy level and reduce the surface defects. Such a bilayer, $\mathrm{SnO}_{2} / \mathrm{PFN}$, as the electron transporting material in inverted $\mathrm{OPVs}\left(\mathrm{ITO} / \mathrm{SnO}_{2} / \mathrm{PFN} /\right.$ PTB7-Th: $\mathrm{PC}_{71} \mathrm{BM} / \mathrm{MoO}_{3} / \mathrm{Ag}$, Fig. 15a) greatly enhanced the efficiency from $4.31 \%$ (pristine $\mathrm{SnO}_{2}$ ) and $9.05 \%$ (pristine $\mathrm{PFN}$ ) to $11.05 \%$ (Fig. 15b). Two main aspects are improved for the bilayer, $\mathrm{SnO}_{2} / \mathrm{PFN}$, compared with the pristine $\mathrm{SnO}_{2}$ and PFN. First, the work function of pristine $\mathrm{SnO}_{2}$ is significantly reduced from $4.46 \mathrm{eV}$ to $3.9 \mathrm{eV}$ because of the dipoles formed on the PFN surface. This significantly reduced work function helps in achieving step-by-step energy level alignment of the device, facilitating electron transport. Furthermore, the transient photovoltage and photocurrent decay curves indicate that the photo-induced electrons can be extracted to the ETL and the lifetime can be extended (Fig. 15c and d). This demonstrates a better extraction capability and a lower charge recombination rate in $\mathrm{SnO}_{2} / \mathrm{PFN}$ compared with both the pristine $\mathrm{SnO}_{2}$ and PFN layers.

Similar results are also found in zwitterion compoundmodified $\mathrm{SnO}_{2}$ films, as reported by Tran et al. ${ }^{229}$ The synergistic effects of aligned energy levels and improved electron extraction and transport contribute to the significantly enhanced efficiency of inverted OPVs. Tran et al. ${ }^{225}$ also reported similar results by selecting alkali carbonates (lithium carbonate, $\mathrm{Li}_{2} \mathrm{CO}_{3}$; potassium carbonate, $\mathrm{K}_{2} \mathrm{CO}_{3}$; and rubidium carbonate, $\mathrm{Rb}_{2} \mathrm{CO}_{3}$ ) to modify the $\mathrm{SnO}_{2}$ surface (Fig. 15e). The reason to choose alkali carbonates is because of their good electron-transporting property but poor hole blocking property. Apart from the enhanced device performance (Fig. 15f), the stability of the unencapsulated device is observed to be very high, maintaining $>90 \%$ of efficiency even after 6 weeks. This stability is because of the inverted device structure and the use of $\mathrm{SnO}_{2}$ as the electron transporting material. The comparison of the stability of devices with $\mathrm{SnO}_{2}$ or $\mathrm{ZnO}$ as the electron transporting material has been reported by other groups of researchers. ${ }^{230}$

In the field of OPVs, non-fullerene acceptors have gained increasing attention recently. Jiang et al. ${ }^{230}$ fabricated inverted non-fullerene OPV devices with the structure of $\mathrm{ITO} / \mathrm{SnO}_{2}$ or ZnO/PM6:IT-4F/molybdenum oxide/silver (ZnO/PM6:IT-4F/ $\left.\mathrm{MoO}_{3} / \mathrm{Ag}\right)$ in which $\mathrm{ZnO}$ or $\mathrm{SnO}_{2}$ is used as the electron transporting material. The device stability study under continuous AM 1.5G illumination indicates that the device fabricated with ZnO displays a dramatic drop in efficiency from $13.0 \%$ (initial) to $5.1 \%$ (after 24 hours), while the device fabricated with $\mathrm{SnO}_{2}$ exhibits a consistent efficiency with slight variation from $14.1 \%$ (initial) to $12.5 \%$ (after 24 hours). It is observed that after 24 hours of illumination, the $\mathrm{ZnO} / \mathrm{IT}-4 \mathrm{~F}$ film on glass decomposed, while the pristine IT- $4 \mathrm{~F}$ and $\mathrm{SnO}_{2} / \mathrm{IT}-4 \mathrm{~F}$ films remained the same. The reason is that the photocatalytic effect of $\mathrm{ZnO}$ caused the decomposition, whereas $\mathrm{SnO}_{2}$ has a wide bandgap that does not allow the absorption of photons, avoiding the photocatalytic effect to cause the decomposition of the active layer material. Meanwhile, an important characteristic of $\mathrm{SnO}_{2}$-based inverted non-fullerene devices is that the efficiency is independent of thermal annealing treatment with a high value of $>12 \%$ at room temperature. This can pave the way for its commercialization in the future.

Another example of inverted non-fullerene OPV with modified $\mathrm{SnO}_{2}$ is reported by Peng et al. ${ }^{231}$ where $\mathrm{InP} / \mathrm{ZnS}$ (indium 
phosphide/zinc chalcogenide) quantum dots are used to passivate the surface defects of $\mathrm{SnO}_{2}$. The reasons for the selection of quantum dots include the following two aspects: (1) the water/alcohol solubility allows a good connection with both $\mathrm{SnO}_{2}$ and the organic active layer and (2) the small size allows distribution into the $\mathrm{SnO}_{2}$ film to passivate the $\mathrm{SnO}_{2}$ surface defects. After modifying with InP/ZnS quantum dots, studies indicate that the transmittance of the device does not change much, which ensures the photon harvesting property. The morphology of the active layer is improved, which is desirable for yielding high $J_{\mathrm{SC}}$ (short-circuit current). Higher SCLC (spacecharge-limited current) mobility favors the FF (fill factor) increment. Semi-logarithmic plots of $J-V$ curves indicate the increased breakdown voltage and slope of the device, which is the origin of higher open circuit voltage $\left(V_{\mathrm{OC}}\right)$. The efficiency of the $\mathrm{SnO}_{2} / \mathrm{InP} / \mathrm{ZnS}$-based device (ITO/SnO $/ 2 / \mathrm{InP} / \mathrm{ZnS} / \mathrm{PM6:Y6/}$ $\mathrm{MoO}_{3} / \mathrm{Ag}$ ) thus reaches a very high value of $15.22 \%$, which is even one of the best efficiencies in non-fullerene OPVs.

An interesting idea is to utilize perovskite nanowires to modify the $\mathrm{SnO}_{2}$ surface to act as the ETL in non-fullerene OPVs. In this context, Zhao et al. ${ }^{232}$ did the same work where $\mathrm{MAPbI}_{3}$ nanowires are introduced on top of $\mathrm{SnO}_{2}$ to make it more compatible with the organic blends in the active layer of the device with the structure of ITO/ $\mathrm{SnO}_{2} / \mathrm{MAPbI}_{3}$ nanowires/ PBDB-T-SF:IT- $4 \mathrm{~F} / \mathrm{MoO}_{3} / \mathrm{Ag}$. Although the efficiency has only a slight increment from $9.53 \%\left(\mathrm{SnO}_{2}\right.$ only) to $10.72 \%$, an interesting phenomenon is that the $\mathrm{MAPbI}_{3}$ bulk filmcontaining device shows only a half efficiency of $5.52 \%$. These results indicate that a suitable perovskite structure can modify the $\mathrm{SnO}_{2}$ and improve the compatibility with the organic blends in non-fullerene OPVs. Therefore, future work on this may be meaningful to improve the efficiency of devices containing both perovskite and non-fullerene active layers for potential commercialization.

These results indicate that the surface modification of $\mathrm{SnO}_{2}$ is an effective strategy for the improvement of OPV device efficiency and stability. Looking for an appropriate material to modify the $\mathrm{SnO}_{2}$ surface is one of the directions for its better use in OPVs. Device efficiency and stability are two of the three crucial parameters (another one is the cost) for OPV device commercialization; therefore, finding a low-cost material to effectively modify $\mathrm{SnO}_{2}$ to improve the device efficiency and stability will greatly pave the way for the future commercialization.

\section{Boosting the perovskite performance and stability through $\mathrm{SnO}_{2}$}

\section{1 $\mathrm{SnO}_{2}$ as the TCO and the ETL for perovskite solar cells (PSCs)}

Metal-halide perovskite thin-film solar cells are typically fabricated using commercial fluorine-doped tin oxide glass as substrates. Although these substrates have low sheet resistance $R_{\mathrm{S}}\left(8-20 \Omega \mathrm{cm}^{2}\right)$, the RMS surface roughness is high $(5-20 \mathrm{~nm})$. Besides, the FTO morphology leads to pinholes in the carrier transport layers. In a study that focused on both FTO and $\mathrm{TiO}_{2}$ blocking layers, Yates et al. ${ }^{233}$ deposited FTO by atmospheric pressure CVD (APCVD) using monobutyl tin trichloride (MBTC) as a tin precursor and aqueous trifluoroacetic acid (TFAA) as the dopant, where two samples with similar sheet resistances but different surface roughness values were deposited by using different deposition thicknesses. For the polycrystalline FTO film, the surface roughness varies with film thickness and develops various crystallographic planes at different rates. Perovskite solar cells (PSCs) with the mesoscopic structure of FTO/TiO ${ }_{2-x} / \mathrm{m}^{-\mathrm{TiO}_{2}} / \mathrm{CH}_{3} \mathrm{CH}_{2} \mathrm{PbI}_{3} /$ spiro-OMeTAD/Au were fabricated using the two types of APCVD FTO and commercial FTO glass substrates. The interesting observation was that even though the commercial FTO (Solaronix TCO22-15) has a lower sheet resistance and a higher carrier mobility, devices fabricated on commercial FTO substrates exhibited lower PCE than those fabricated on APCVD-grown FTO substrates. This difference was attributed to the reduction of optical transmission due to increased absorption by free carriers of transparent electrode layers. The higher surface roughness of APCVD FTO yields slightly higher PCE because of increased internal light scattering.

To gain a deeper insight into the effect of the TCO layer on PSCs, Afzaal et al. used APCVD to deposit FTO films with different thicknesses and a fixed resistance $\left(R_{\mathrm{S}}\right)$ as well as different $R_{\mathrm{S}}$ values for the same thickness. ${ }^{235}$ Mesoscopic PSC thin-film solar cells $(1 \mathrm{~cm} \times 1 \mathrm{~cm})$ with the structure FTO/ $\mathrm{TiO}_{2-x} / \mathrm{CH}_{3} \mathrm{CH}_{2} \mathrm{PbI}_{3} /$ spiro-OMeTAD/Au were fabricated on APCVD-grown FTO substrates. It was found that increasing FTO thickness has a clear and significant effect on the PCE of the devices due to the correlation between the FTO film thickness and its surface roughness. Samples with thicker FTO layers but the same $R_{\mathrm{S}}$ show improved FF and reduced $V_{\mathrm{OC}}$ and $J_{\mathrm{SC}}$. When $R_{\mathrm{S}}$ increased, with the thickness remaining the same, both $V_{\mathrm{OC}}$ and $J_{\mathrm{SC}}$ increased, but FF was reduced. For the optimized FTO deposition, a PCE of $17.8 \%$ was achieved, higher than that of the reference device fabricated on commercial FTO substrates. This study demonstrates that careful tailoring of the optical, structural, and electronic properties of FTO is necessary to obtain the enhanced performance of PSC devices. $\mathrm{SnO}_{2}$ and different metal-doped $\mathrm{SnO}_{2}$ are used as the electron transport layer (ETL) in planar PSCs. Different approaches such as the atomic layer deposition (ALD) technique, solution-processing method, and the CBD technique have been adopted for the preparation of this $\mathrm{SnO}_{2}$ based ETL. Baena et al. ${ }^{234}$ reported planar PSCs using $\mathrm{SnO}_{2}$ and $\mathrm{TiO}_{2}$ as ETLs and they found that, for planar PSCs, $\mathrm{SnO}_{2}$ was much more effective than $\mathrm{TiO}_{2}$. They claimed that $\mathrm{SnO}_{2}$ has a much more favorable conduction band alignment with the $\mathrm{MAPbI}_{3}$ and mixed $\left(\mathrm{FAPbI}_{3}\right)_{0.85}(-$ $\left.\mathrm{MAPbBr}_{3}\right)_{0.15}$ perovskite absorber, which yielded a much higher PCE as shown in Fig. 16a-d, using $\mathrm{SnO}_{2}$ as the electron selective layer. In this report, the ALD technique was used to prepare the $\mathrm{SnO}_{2}$ layer on the top of an FTO substrate. In parallel, Ke et al. also reported a planar $\mathrm{MaPbI}_{3}$ PSC using solution-processed $\mathrm{SnO}_{2}$ as the ETL. ${ }^{236}$ They prepared $\mathrm{SnO}_{2}$ by facile spin coating of the $\mathrm{SnCl}_{2} \cdot 2 \mathrm{H}_{2} \mathrm{O}$ precursor prepared at room temperature, followed by thermal annealing in air at $180^{\circ} \mathrm{C}$ for $1 \mathrm{~h}$. The mild 


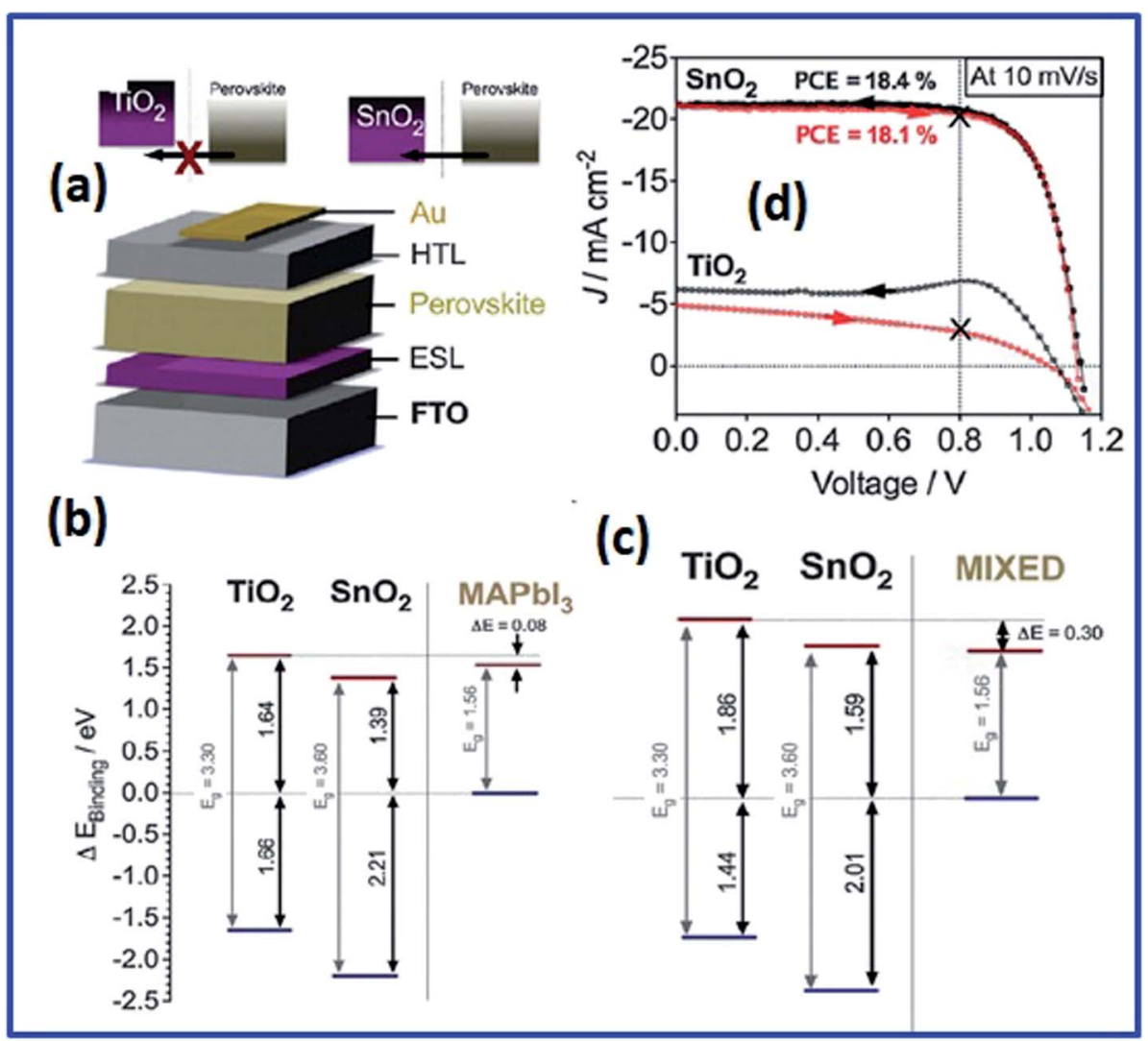

Fig. 16 (a) Energy level diagrams and electron injection characteristics of $\mathrm{SnO}_{2}$ and $\mathrm{TiO}_{2}$-based planar PSCs. Schematic conduction band diagram of the perovskite films and the electron selective layers ( $\mathrm{ESL}$ ), $\mathrm{TiO}_{2}$ and $\mathrm{SnO}_{2}$ for (b) $\mathrm{MAPbl}_{3}$ and $(\mathrm{c})\left(\mathrm{FAPb}_{3}\right)_{0.85}\left(\mathrm{MAPbBr}_{3}\right)_{0.15}, \mathrm{labeled}_{3}$ 'mixed'. (d) Current-voltage characteristics of planar perovskite devices based on $\mathrm{SnO}_{2}$ and $\mathrm{TiO}_{2} \mathrm{ESLs}^{234}$ presented with permission and copyright.

annealing conditions resulted in a smooth nanocrystalline $\mathrm{SnO}_{2}$ ETL which covers the FTO substrate and improves its optical transmittance. They achieved high performance planar PSCs with a forward scan PCE of $14.82 \%$ and a reverse scan efficiency of $17.21 \%$. After that several groups had reported planar PSCs based on $\mathrm{SnO}_{2}$ as the ETL. ${ }^{53,237-240}$ Different approaches were made for optimizing the $\mathrm{SnO}_{2}$ layer as the ETL to boost the performance of the PSCs. Among these approaches, the most effective approaches are doping of $\mathrm{SnO}_{2}$ using different metals such as aluminium (Al), antimony (Sb), lithium (Li), and tantalum (Ta); ; ${ }^{56,241,242}$ high temperature annealing; $;{ }^{243}$ use of different additives such as ethylenediaminetetraacetic acid (EDTA) ${ }^{65}$ and 2,2,2-trifluoroethanol in $\mathrm{SnO}_{2}{ }^{244}$ and an additional ETL such as $\mathrm{PCBM}^{239}$ or $\mathrm{In}_{2} \mathrm{O}_{3}$ together with the $\mathrm{SnO}_{2}$ layer. ${ }^{240}$ With all these approaches, the device performance could be significantly improved.

Although the PCE of present perovskite thin-film solar cells is well over $20 \%$, the $J-V$ characteristics of these devices are scan direction-dependent and show considerable hysteresis. ${ }^{245,246}$ Earlier, this phenomenon was thought to be due to charge accumulation effects at the $\mathrm{TiO}_{2} /$ perovskite interface where $\mathrm{TiO}_{2}$ is the default electron transport layer (ETL). ${ }^{245}$ To eliminate the hysteresis, interfacial layers based on fullerene derivatives have been inserted between the ETL and the perovskite layer. ${ }^{238,246}$ The hysteresis behavior in $J-V$ characteristics is also a burden in the case of $\mathrm{SnO}_{2}$ as the ETL. Recently, Jung et al. employed a sol-gel method to deposit the $\mathrm{SnO}_{2}$ ETL onto FTO substrates using tin(Iv) isopropoxide as the precursor. ${ }^{243}$ The process parameters investigated include the tin precursor concentration and post-deposition annealing temperatures. The thermal annealing was in addition to the heating in a $\mathrm{N}_{2}$ glovebox environment that was intended for drying the deposited layer. The $\mathrm{SnO}_{2}$ ETL annealing temperature in the range of $100{ }^{\circ} \mathrm{C}$ to $500{ }^{\circ} \mathrm{C}$ has a significant effect on lowering the hysteresis of the $J-V$ profiles. From $100{ }^{\circ} \mathrm{C}$ to $250{ }^{\circ} \mathrm{C}$, the hysteresis between the forward and reverse $J-V$ scans decreases and then increases again from $250{ }^{\circ} \mathrm{C}$ to $500{ }^{\circ} \mathrm{C}$. Annealing at $250^{\circ} \mathrm{C}$ was found to be more effective as the ultraviolet photoelectron spectroscopy (UPS) study showed a better band alignment with the electrode (Fig. 17a). At $250^{\circ} \mathrm{C}$, the PV parameters for the forward and reverse scans were almost the same. An average PCE of $16.08 \%$ was achieved for a precursor concentration of $0.1 \mathrm{M}$ (Fig. 17c), and this was further improved to $19.17 \%$ with the incorporation of potassium in the perovskite (Fig. 17d). The greatly reduced hysteresis was attributed to both a reduced interfacial capacitance and faster stabilization of the photocurrent. PSCs with mixed cation and anion absorbers with the device geometry of $\mathrm{FTO} / \mathrm{SnO}_{2} /\left(\mathrm{FAPbI}_{3}\right)_{0.873}\left(\mathrm{CsPbBr}_{3}\right)_{0.125} /$ 


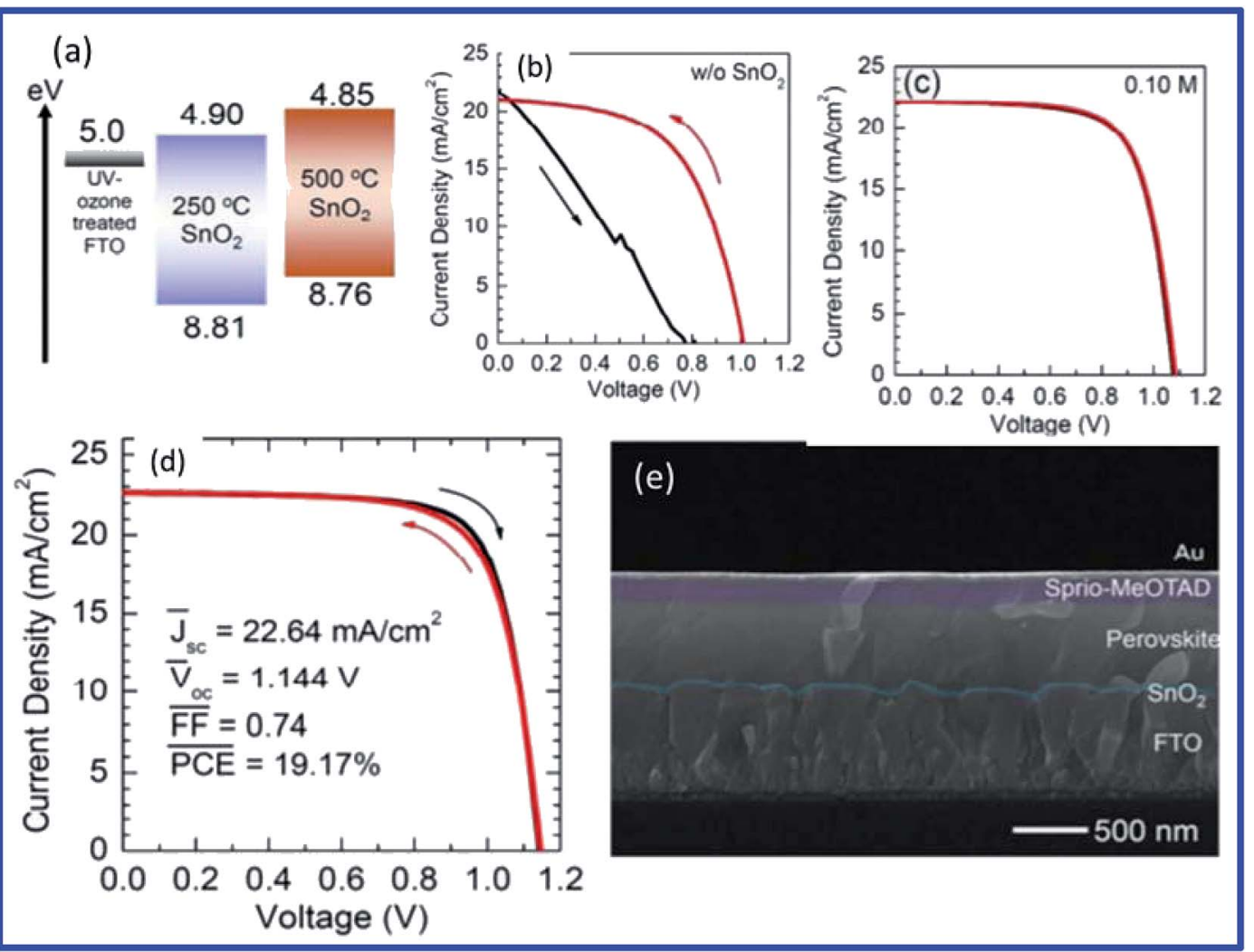

Fig. 17 (a) Schematic of energy alignment of the UVO treated $\mathrm{FTO}$ and $\mathrm{SnO}_{2}$ films annealed at $250^{\circ} \mathrm{C}$ and $500{ }^{\circ} \mathrm{C}$. Reverse (red) scan and forward (black) scan J-V curves of planar perovskite solar cells (b) without and (c) with $\mathrm{SnO}_{2}$ thin films prepared from $0.1 \mathrm{M} \mathrm{concentration} \mathrm{of} \mathrm{the} \mathrm{SnO}_{2}$ precursor solution. (d) J-V curves and (e) cross-sectional SEM image of the best performing planar perovskite (with potassium doped) solar cell employing the $0.1 \mathrm{M}$-based and $250{ }^{\circ} \mathrm{C}$-annealed $\mathrm{SnO}_{2}$ thin film with a thickness of $\sim 40 \mathrm{~nm},{ }^{243}$ presented with permission and copyright.

spiro-OMeTAD/Au (Fig. 17e) were fabricated. The effect of precursor concentration for $\mathrm{SnO}_{2}$ films annealed at $250{ }^{\circ} \mathrm{C}$ was also studied, ${ }^{243}$ which mainly influenced the thickness of the spin-coated layer. By varying the annealing temperature and the precursor concentration, the hysteresis area under the forward and backward $J-V$ curves was eliminated (Fig. 17c and d). For concentrations above and below the optimal value of $0.1 \mathrm{M}$, there was significant hysteresis and the hysteresis was the largest for controlled devices where the $\mathrm{SnO}_{2}$ layer was not used (Fig. 17b). For the low concentration of the precursor, the hysteresis was ascribed to the poor surface coverage of the FTO substrate, while for high concentrations, the hysteresis was due to the increased series resistance. This study highlights the importance of post-deposition annealing of $\mathrm{SnO}_{2}$ ETL in eliminating the hysteresis phenomenon in the $J-V$ characteristics of perovskite solar cells.

It was also found that the low open-circuit voltage (those with a bandgap of between 1.59 and 1.63) originates from electron collecting layers (mainly oxide nanocrystal thin films) used as transparent electrodes. A well-matched energy band between the ETL and perovskites improves $V_{\text {OC }}$ by avoiding the excessive band offset while accelerating the charge carrier extraction, resulting in efficient transport. In this regard, very recently, Wang et al. ${ }^{\mathbf{2 4 0}}$ reported a unique work based on gradient energy alignment engineering using the $\operatorname{In}_{2} \mathrm{O}_{3} / \mathrm{SnO}_{2}$ bilayer as the ETL and found a negligent extent of hysteresis loss in $V_{\mathrm{OC}}$. Deposition of a very thin layer of $\mathrm{Li}$ on the $\mathrm{SnO}_{2}$ layer as the ETL was also found to be very effective for proper band alignment and to reduce surface defects at the interface in the case of all inorganic perovskite $\mathrm{CsPbI}_{3-x} \mathrm{Br}_{x}$ solar cells (Fig. 18ac). ${ }^{239}$ Low-temperature solution-processed $\mathrm{SnO}_{2}$ nanoparticles were also found to be very efficient as the ETL for planar perovskite solar cells on a rigid substrate and a flexible PET substrate (Fig. 18d and e). ${ }^{247}$

The use of the $\mathrm{SnO}_{2}$ layer on a CVD-/sputter-grown FTO substrate could broaden the optical transmission band of the electron selective layer and fill electronic states in the conduction band of the perovskite film resulting in the enhancement of charge concentration in quasi-Fermi energy levels at ETLperovskite interfaces. Solution-processed $\mathrm{Al} / \mathrm{F} / \mathrm{Sb}$-doped tin oxide coating is a feasible way to produce transparent conductive substrates and electron transport layers in a single process that will be crucial for developing hysteresis-free highperformance PSC (PCE $=16-20 \%$ ) modules on the industrial scale. ${ }^{247}$

\subsection{Impact of $\mathrm{SnO}_{2}$ on flexible PSCs}

Since the first report of flexible PSCs with a PCE of $2.62 \%$ by Mathews' group in $2013,{ }^{248}$ the PCEs of the flexible PSCs have increased to $18.5 \% .^{249,250}$ Progress of the flexible PSCs was based 


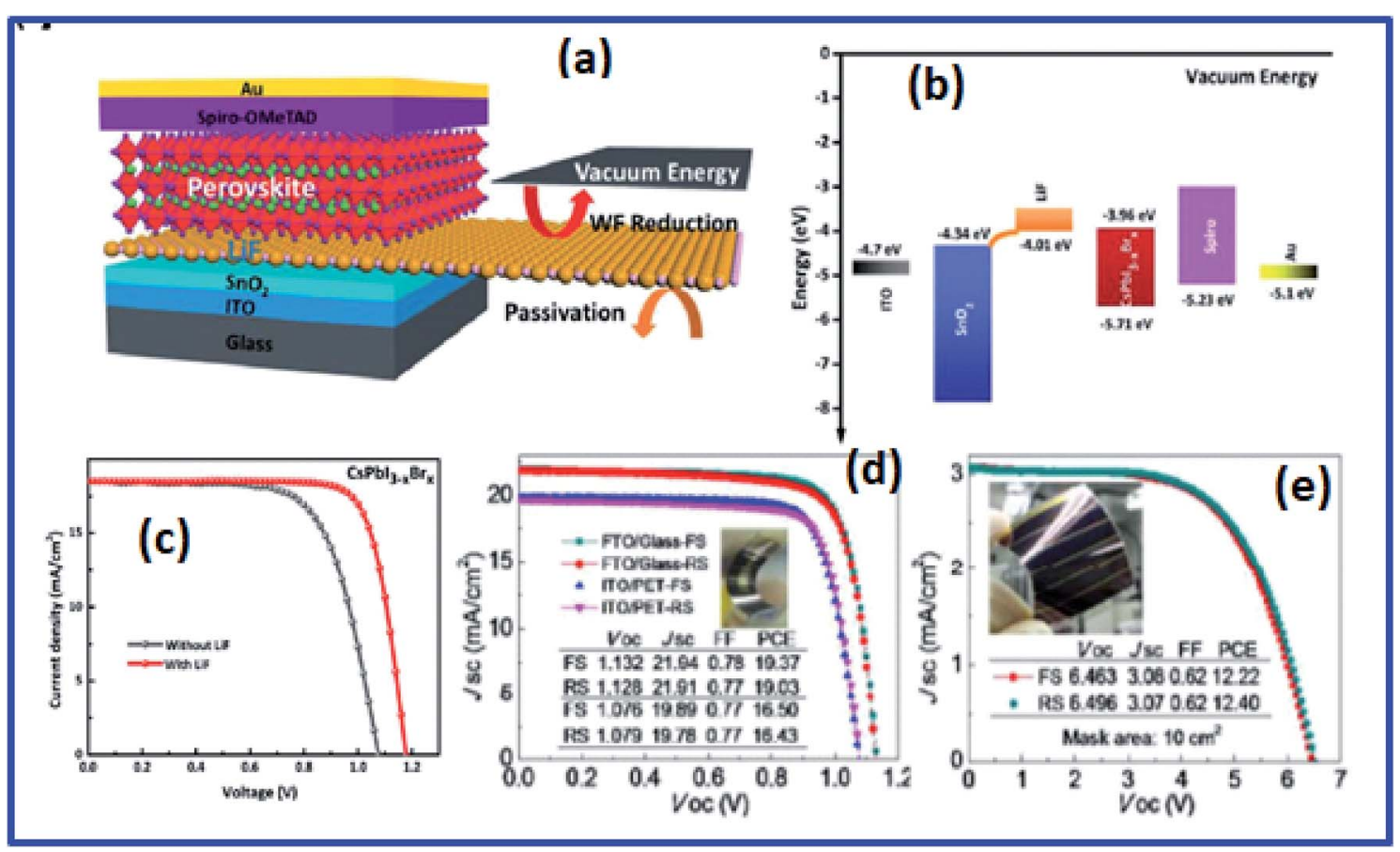

Fig. 18 (a) Scheme of the device architecture of the inorganic perovskite solar cells; LiF was used to modify the $\mathrm{SnO}_{2}$ surface. (b) Energy band alignment for each layer in $\mathrm{CsPb}_{3-x} \mathrm{Br}_{x}$ inorganic solar cells. (c) $\mathrm{J}-\mathrm{V}$ characteristics of inorganic $\mathrm{CsPb}_{3-x} \mathrm{Br}_{x}$ solar cells with and without the LiF interlayer. $^{239}$ (d) Hysteresis-free perovskite devices on glass and PET substrates and (e) flexible perovskite module of the same devices by chemical treatment of the $\mathrm{SnO}_{2}$ layer, ${ }^{247}$ presented with permission and copyright.

on different functional layers, which have been prepared by different low temperature process technologies. In this regard, $\mathrm{SnO}_{2}$ was found to be very effective as the ETL, which has been prepared by different techniques such as solution-processing, room temperature sputtering, ALD and slot-die printing. Dong et al. ${ }^{63}$ reported the in situ regrowth of the $\mathrm{SnO}_{2}$ layer by pretreating the substrate by ultraviolet ozone (UVO). This UVO treatment helped the substrate to adsorb the optimum amount of water molecules which facilitates hydrolysis-condensation reactions for the regrowth of the spin-coated $\mathrm{SnO}_{2} \mathrm{NPs}$. With this deposition technique of the $\mathrm{SnO}_{2}$ as the ETL, the authors reported a PCE of $17.5 \%$ for a flexible PSC. ${ }^{63}$ Qiu et al. ${ }^{251}$ reported a two-step solution-processed $\mathrm{SnO}_{2}$ layer formation by depositing a layer of $\mathrm{SnCl}_{2}$ on the spin-coated layer of $\mathrm{SnO}_{2} \mathrm{NPs}$. This $\mathrm{SnCl}_{2}$ layer helped to improve the quality of the ETL through hydrolysis and oxidation of $\mathrm{SnCl}_{2}$. They have reported the device efficiency of $15.21 \%$ for the flexible PSC devices, with prolonged shelf-stability. ${ }^{251}$ Liu et al. ${ }^{252}$ also reported two-step techniques to deposit the $\mathrm{SnO}_{2}$ layer. After depositing a $\mathrm{SnO}_{2}$ NP aqueous solution on an ITO/polyethylene naphthalate, PEN substrate by spin coating, they treated the film by the hydrothermal technique using an autoclave at $100{ }^{\circ} \mathrm{C}$ for $1 \mathrm{~h}$, and a significant improvement of the quality of the $\mathrm{SnO}_{2}$ layer was obtained. They achieved a certified efficiency of $17.27 \%$ for the flexible PSCs. The authors concluded that the enhanced performance of the PSCs can be attributed to the large grain sizes of the perovskite on hydrothermally treated $\mathrm{SnO}_{2}$, low trap density of the device, high charge transport efficiency of hydrothermally treated $\mathrm{SnO}_{2}$, and the better energy band matching between hydrothermally treated $\mathrm{SnO}_{2}$ and the perovskite layer. ${ }^{252}$ In most of the reports, spin coating of an aqueous solution of $\mathrm{SnO}_{2}$ NPs followed by some kind of treatment have been used for a better quality of the $\mathrm{SnO}_{2}$ layer. The work by Yang et al. ${ }^{65}$ revealed that by preparing a complex of $\mathrm{SnO}_{2}$ aqueous solution with EDTA, an effective ETL can be formed. It has been found that this EDTA-complexed $\mathrm{SnO}_{2}$ is better matched with the conduction band of perovskite, leading to high $V_{\mathrm{OC}}$. Its electron mobility is about three times larger than that of $\mathrm{SnO}_{2}$. Using EDTA-complexed $\mathrm{SnO}_{2}$, the group has reported an efficiency of $18.28 \%$ for a flexible device.$^{65}$ Similarly, Kam et al. ${ }^{31}$ reported room-temperature RF sputtered $\mathrm{SnO}_{2}$ as a promising ETL with a suitable band structure, high transmittance, and excellent stability for the PSCs. They achieved PCEs of $12.82 \%$ and $5.88 \%$ on a rigid glass substrate and a flexible PEN substrate, respectively. ${ }^{31}$ Another non-solution based process which has been reported for the formation of the $\mathrm{SnO}_{2}$ layer is the slot die coating. The approach towards flexible perovskite solar cell modules (PSCMs) was reported by $\mathrm{Bu}$ et al. ${ }^{253}$ using $\mathrm{SnO}_{2}$ as the ETL. They reported flexible PSCMs on an ITO/PET substrate using triple cation perovskite and the slot-die printed $\mathrm{SnO}_{2}$ layer as the ETL. They provided a facile interface passivation strategy with potassium pre-treatment for $\mathrm{SnO}_{2}$ ETLs to obtain hysteresis-free high efficiency devices and reported small size flexible PSCs with an efficiency of $17.18 \%$ and large size $\left(5 \times 6 \mathrm{~cm}^{2}\right)$ flexible modules with an efficiency over $15 \%$ (Fig. 19a-c). ${ }^{253}$

$\mathrm{SnO}_{2}$ was also found to be very effective as an electron selective contact in the case of 2-terminal monolithic tandem 


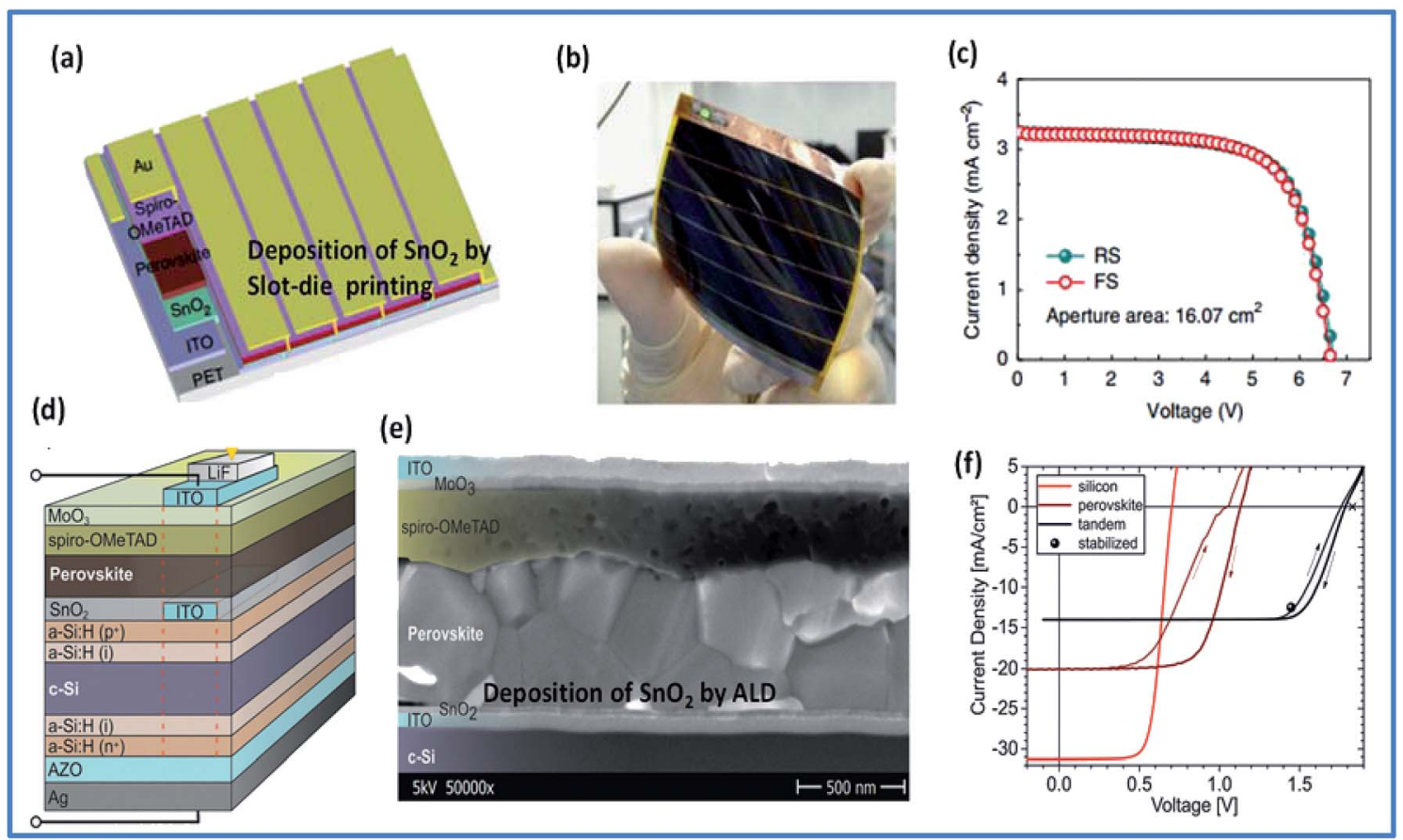

Fig. 19 (a) Structure of the 6 sections of series-connected large-area flexible PSCMs. (b) Photograph of the flexible PSCM and (c) the corresponding $J-V$ curves of the champion flexible PSCM. ${ }^{253}$ (d) Schematic device design of the silicon heterojunction/perovskite tandem solar cell. The red dashed line indicates the active area. (e) Cross-sectional scanning electron micrograph of a typical monolithic tandem solar cell. (f) $J-V$ characteristics of tandem, perovskite standalone and Si standalone cells, ${ }^{254}$ presented with permission and copyright.

solar cells. A low temperature processed electron selective layer is highly commended since the bottom cell of the tandem structure could be damaged by the high-temperature process. Albrecht et al. ${ }^{254}$ reported a 2-terminal monolithic tandem solar cell using Si as the bottom cell, PSCs as the top cell and ALDgrown $\mathrm{SnO}_{2}$ as the electron selective contact and achieved an efficiency of $19.9 \%$ with a $V_{\mathrm{OC}}$ of $1785 \mathrm{mV}$ (Fig. 19d-f).

Liang et al. investigated a new arrangement of OMO structure, tungsten oxide, and $\mathrm{WO}_{3}(23 \mathrm{~nm}) / \mathrm{Ag}(10.7 \mathrm{~nm}) / \mathrm{SnO}_{2}(35$ $\mathrm{nm}$ ) with the lowest sheet resistance and high visible transmittance. ${ }^{255}$ The device conversion efficiency boosted to $14 \%$ with a high $J_{\mathrm{SC}}\left(7.9 \mathrm{~mA} \mathrm{~cm}{ }^{-2}\right.$ to $\left.18.6 \mathrm{~mA} \mathrm{~cm}^{-2}\right)$ and a high $\mathrm{FF}$ from 0.24 to 0.72 due to the $\mathrm{SnO}_{2} \mathrm{NP}$ interfacial layer. ${ }^{255}$ This work suggested a big potential of OMO in the replacement of ITO in flexible solar cell applications. Similarly, Hashemi et al. reported different approaches to develop flexible and wearable solar cells for self-powered wearable devices. ${ }^{256}$ Table 5 compares the device performances of perovskite based solar cells for doped $\mathrm{SnO}_{2}$.

\subsection{Plasma treated $\mathrm{SnO}_{2}$ for PSCs}

Over the past few decades, plasma processing has become a tool for various manufacturing and materials processing industries. Plasma technologies have found applications in aerospace, automotive, steel, biomedical, and toxic waste management industries. ${ }^{289}$ Most importantly, plasma processing is now well adopted in large scale integrated circuit manufacturing. The primary properties of plasmas that have attracted the attention of industries include high power and energy density of thermal plasmas used in additive manufacturing and the abundant presence of reactive species capable of initiating chemical reactions on their own. ${ }^{290}$ These reactive species can perform complex transformations that are in many ways beyond the scope of other conventional techniques. One of the major advantages of using plasma technologies, in particular, for oxide semiconductors is that these processes are dry and environmentally friendly. In this sub-section, we emphasize on nonthermal plasma induced processing of $\mathrm{SnO}_{2}$ nanostructures. Non-thermal plasmas are advantageous as they can be operated at low temperatures and provide unique opportunities for lowtemperature material processing.

Plasma-based processes are indispensable in reactive sputtering and plasma-enhanced CVD. These techniques are widely used for the synthesis of high-quality $\mathrm{SnO}_{2}$ nanostructures that are used for a varied range of applications. ${ }^{291-293}$ In this context, Tarlov and Evans were among the first to study the effect of plasma functionalization on $\mathrm{SnO}_{2}$ thin films. ${ }^{294}$ They demonstrated that RF water plasma treatment of $\mathrm{SnO}_{2}$ films is an excellent method for preparing "clean", hydroxylated surfaces. They studied the hydroxylated surfaces using angle-resolved Xray photoelectron spectroscopy and electron energy loss spectroscopy, to identify the changes in surface electronic states and chemical properties. Interestingly, the plasma treatment removed the oxygen vacancies by restoring the $\mathrm{Sn}^{4+}$ valency in the surface region. In general, plasma treatment also removed unwanted carbon contaminants with no preferential surface hydroxylation. This study reintroduced plasma-based systems as a new synthesizing route for oxides in general and in 
Table 5 Dopants/foreign elements present in the $\mathrm{SnO}_{2}$ layer and the corresponding PSC device parameters ${ }^{a}$

\begin{tabular}{|c|c|c|c|c|c|c|c|}
\hline Method & Device layer $\left(\mathrm{SnO}_{2}\right)$ & $\begin{array}{l}\text { Doping/foreign element(s) } \\
\left(\mathrm{SnO}_{2}\right)\end{array}$ & $V_{\mathrm{OC}}(\mathrm{V})$ & $\begin{array}{l}J_{\mathrm{SC}}(\mathrm{mA} \\
\left.\mathrm{cm}^{-2}\right)\end{array}$ & FF $(\%)$ & $\begin{array}{l}\text { PCE } \\
(\%)\end{array}$ & Reference \\
\hline Sol-gel & $\begin{array}{l}\mathrm{FTO} / \mathrm{SnO}_{2}(\mathrm{Zr} / \mathrm{F}) / \\
\mathrm{CH}_{3} \mathrm{NH}_{3} \mathrm{PbI}_{3} / \mathrm{SiGe} / \text { spiro- } \\
\text { OMeTAD/Au }\end{array}$ & $\mathrm{Zr} / \mathrm{F}$ & 1.105 & 24.39 & 71.2 & 19.19 & Tian et al. ${ }^{257}$ \\
\hline Solvothermal & $\mathrm{SnO}_{2}-\mathrm{TiO}_{2}$ & $\mathrm{TiO}_{2}$ & 0.76 & 7.18 & 60.41 & 3.28 & $\begin{array}{l}\text { Qureshi } \\
\text { et al. }\end{array}$ \\
\hline Spinning & $\begin{array}{l}\mathrm{FTO} / \mathrm{Cu}-\mathrm{SnO}_{2} / \\
\mathrm{CH}_{3} \mathrm{NH}_{3} \mathrm{PbI}_{3} / \text { spiro- } \\
\text { OMeTAD/Au }\end{array}$ & $\mathrm{Cu}$ & 0.78 & 21.74 & 0.509 & 8.48 & Li et $a l . .^{259}$ \\
\hline Spinning & $\begin{array}{l}\mathrm{FTO} / \mathrm{Zn}-\mathrm{SnO}_{2} / \\
\mathrm{CH}_{3} \mathrm{NH}_{3} \mathrm{PbI}_{3} / \text { spiro- } \\
\text { OMeTAD/Au }\end{array}$ & $\mathrm{Zn}$ & 0.97 & 22.24 & 0.610 & 13.17 & Li et al. ${ }^{259}$ \\
\hline Spinning & $\begin{array}{l}\mathrm{FTO} / \mathrm{Cd}-\mathrm{SnO}_{2} / \\
\mathrm{CH}_{3} \mathrm{NH}_{3} \mathrm{PbI}_{3} / \text { spiro- } \\
\text { OMeTAD/Au }\end{array}$ & $\mathrm{Cd}$ & 0.90 & 20.56 & 0.487 & 9.03 & Li et al. ${ }^{259}$ \\
\hline Spinning & $\begin{array}{l}\mathrm{FTO} / \mathrm{Li}-\mathrm{SnO}_{2} / \mathrm{CH}_{3} \mathrm{NH}_{3} \mathrm{PbI}_{3} / \\
\text { spiro-OMeTAD/Au }\end{array}$ & $\mathrm{Li}$ & 0.46 & 18.99 & 0.423 & 3.76 & Li et $a l^{259}$ \\
\hline Spinning & $\begin{array}{l}\mathrm{FTO} / \mathrm{Ti}-\mathrm{SnO}_{2} / \mathrm{CH}_{3} \mathrm{NH}_{3} \mathrm{PbI}_{3} / \\
\text { spiro-OMeTAD/Au }\end{array}$ & $\mathrm{Ti}$ & 1010 & 21.49 & 66.6 & 14.45 & Li et $a l .{ }^{259}$ \\
\hline $\begin{array}{l}\text { Doctor blade } \\
\text { technique }\end{array}$ & $\mathrm{SnO}_{2}-\mathrm{ZnO}$ & $\mathrm{ZnO}$ & 6.38 & 0.60 & 0.46 & 1.75 & $\begin{array}{l}\text { Sujinnapram } \\
\text { et al. }{ }^{260}\end{array}$ \\
\hline Sol-gel & ITO/Li: $\mathrm{SnO}_{2}$ & ITO/Li & 1.10 & 23.27 & 70.71 & 18.20 & Park et $a l .^{241}$ \\
\hline Sol-gel & $\mathrm{SnO}_{2} / \mathrm{NH}_{4} \mathrm{Cl}-\mathrm{SnO}_{2}$ & $\mathrm{NH}_{4} \mathrm{Cl}$ & 1.10 & 24.37 & 78.31 & 21.01 & Song et al. ${ }^{261}$ \\
\hline Sol-gel & $\mathrm{SnO}_{2}-\mathrm{CNT}$ & CNT & 1.12 & 23.26 & 78.23 & 20.33 & Tang et al. ${ }^{262}$ \\
\hline $\begin{array}{l}\text { Magnetron } \\
\text { sputtering }\end{array}$ & $\mathrm{FTO} / \mathrm{SnO}_{2}\left(\mathrm{Ar}: \mathrm{O}_{2}\right)$ & FTO & 1.05 & 22.45 & 0.76 & 18.20 & Bai et al. ${ }^{263}$ \\
\hline Spin-coating & $\mathrm{SnO}_{2} / \mathrm{PFN}-\mathrm{Br}$ & PFN-Br & 1.09 & 23.46 & 77.2 & 19.77 & Guo et al. ${ }^{264}$ \\
\hline Sol-gel & $\begin{array}{l}\mathrm{SnO}_{2} / \mathrm{CH}_{3} \mathrm{NH}_{3} \mathrm{PbI}_{3} / \mathrm{SiGe} / \\
\text { spiro-OMeTAD/SiGeSn }\end{array}$ & $\begin{array}{l}\mathrm{CH}_{3} \mathrm{NH}_{3} \mathrm{PbI}_{3} / \mathrm{SiGe} / \text { spiro- } \\
\text { OMeTAD/SiGeSn }\end{array}$ & 0.78 & 49.75 & 73.4 & 28.57 & $\begin{array}{l}\text { Kumar } \\
\text { et al. }\end{array}$ \\
\hline Spin-coating & $\begin{array}{l}\mathrm{ITO} / \mathrm{SnO}_{2} / \mathrm{CH}_{3} \mathrm{NH}_{3} \mathrm{PbI}_{3} / \\
\mathrm{SiGe} / \text { spiro-OMeTAD/Ag }\end{array}$ & $\begin{array}{l}\mathrm{CH}_{3} \mathrm{NH}_{3} \mathrm{PbI}_{3} / \mathrm{SiGe} / \text { spiro- } \\
\text { OMeTAD/Ag }\end{array}$ & 1.08 & 19.5 & 0.62 & 13 & Song et al. ${ }^{266}$ \\
\hline Sol-gel & $\begin{array}{l}\mathrm{FTO} / \mathrm{SnO}_{2} / \mathrm{CH}_{3} \mathrm{NH}_{3} \mathrm{PbI}_{3} / \\
\mathrm{SiGe} / \text { spiro-OMeTAD/Au }\end{array}$ & $\begin{array}{l}\mathrm{CH}_{3} \mathrm{NH}_{3} \mathrm{PbI}_{3} / \mathrm{SiGe} / \text { spiro- } \\
\mathrm{OMeTAD} / \mathrm{Au}\end{array}$ & 1.11 & 23.27 & 0.67 & 17.21 & Ke et $a l^{236}$ \\
\hline Sol-gel & $\begin{array}{l}\mathrm{ITO} / \mathrm{SnO}_{2} / \mathrm{CH}_{3} \mathrm{NH}_{3} \mathrm{PbI}_{3} / \\
\text { SiGe/spiro-OMeTAD/Au }\end{array}$ & $\begin{array}{l}\mathrm{CH}_{3} \mathrm{NH}_{3} \mathrm{PbI}_{3} / \mathrm{SiGe} / \text { spiro- } \\
\text { OMeTAD/Au }\end{array}$ & 1.084 & 21.98 & 64.17 & 15.29 & Park et al. ${ }^{241}$ \\
\hline Sol-gel & $\mathrm{PFT} / \mathrm{ITO} / \mathrm{SnO}_{2} / \mathrm{meso}^{-\mathrm{TiO}_{2}}$ & Meso- $\mathrm{TiO}_{2}$ & 20.70 & 1.036 & 65.55 & 14.07 & $\begin{array}{l}\text { Dagar } \\
\text { et al. }\end{array}$ \\
\hline Sol-gel & $\begin{array}{l}\mathrm{FTO} / \mathrm{SnO}_{2} \mathrm{ESL} / \\
\mathrm{CH}_{3} \mathrm{NH}_{3} \mathrm{PbI}_{3} / \text { spiro- } \\
\text { OMeTAD/Au }\end{array}$ & $\begin{array}{l}\mathrm{CH}_{3} \mathrm{NH}_{3} \mathrm{PbI}_{3} / \text { spiro- } \\
\text { OMeTAD/Au }\end{array}$ & 1.09 & 18.48 & 75.04 & 15.10 & Ke et $a .^{268}$ \\
\hline Sol-gel & $\begin{array}{l}\text { Glass/FTO/SnO } / \\
\mathrm{CH}_{3} \mathrm{NH}_{3} \mathrm{PbI}_{3} / \text { spiro/Au } \\
\left(\mathrm{UVLT}_{-} \mathrm{SnO}_{2}\right)\end{array}$ & $\begin{array}{l}\mathrm{CH}_{3} \mathrm{NH}_{3} \mathrm{PbI}_{3} / \text { spiro/Au } \\
\left(\mathrm{UVLT}-\mathrm{SnO}_{2} \text { ) }\right.\end{array}$ & 1.06 & 21.94 & 61.73 & 14.36 & $\begin{array}{l}\text { Huang } \\
\text { et al. }\end{array}$ \\
\hline Sol-gel & $\begin{array}{l}\text { Glass/FTO/SnO } / \\
\mathrm{CH}_{3} \mathrm{NH}_{3} \mathrm{PbI}_{3} / \text { spiro/Au (HT- } \\
\left.\mathrm{SnO}_{2}\right)\end{array}$ & $\begin{array}{l}\mathrm{CH}_{3} \mathrm{NH}_{3} \mathrm{PbI}_{3} / \text { spiro/Au (HT- } \\
\left.\mathrm{SnO}_{2}\right)\end{array}$ & 1.06 & 19.82 & 57.40 & 11.49 & $\begin{array}{l}\text { Huang } \\
\text { et al. }\end{array}$ \\
\hline Spin-coating & $\begin{array}{l}\mathrm{FTO} / \mathrm{SnO}{ }_{2} / \mathrm{CH}_{3} \mathrm{NH}_{3} \mathrm{PbI}_{3} / \\
\mathrm{CuSCN} / \mathrm{Au} \text { (water) }\end{array}$ & $\begin{array}{l}\mathrm{CH}_{3} \mathrm{NH}_{3} \mathrm{PbI}_{3} / \mathrm{CuSCN} / \mathrm{Au} \\
\text { (water) }\end{array}$ & 0.95 & 18.19 & 0.38 & 6.59 & $\begin{array}{l}\text { Murugadoss } \\
\text { et al. }\end{array}$ \\
\hline Spin-coating & $\begin{array}{l}\mathrm{FTO} / \mathrm{SnO}_{2} / \mathrm{CH}_{3} \mathrm{NH}_{3} \mathrm{PbI}_{3} / \\
\mathrm{CuSCN} / \mathrm{Au} \text { (ethanol) }\end{array}$ & $\begin{array}{l}\mathrm{CH}_{3} \mathrm{NH}_{3} \mathrm{PbI}_{3} / \mathrm{CuSCN} / \mathrm{Au} \\
\text { (ethanol) }\end{array}$ & 0.96 & 18.99 & 45 & 8.38 & $\begin{array}{l}\text { Murugadoss } \\
\text { et al. }{ }^{270}\end{array}$ \\
\hline Sol-gel & $\begin{array}{l}\mathrm{FTO} / \mathrm{SnO}_{2} / \mathrm{CH}_{3} \mathrm{NH}_{3} \mathrm{PbI}_{3} / \\
\text { spiro-OMeTAD/G }\end{array}$ & $\begin{array}{l}\mathrm{CH}_{3} \mathrm{NH}_{3} \mathrm{PbI}_{3} / \text { spiro- } \\
\text { OMeTAD/G }\end{array}$ & 1.05 & 22.78 & 78 & 18.65 & $\begin{array}{l}\text { Zhang } \\
\text { et al. }\end{array}$ \\
\hline Sol-gel & $\begin{array}{l}\mathrm{FTO} / \mathrm{CL} / \mathrm{SnO}_{2} / \mathrm{TiO}_{2} / \\
\mathrm{CH}_{3} \mathrm{NH}_{3} \mathrm{PbI}_{3} / \mathrm{HTM} / \mathrm{Au}\end{array}$ & $\mathrm{TiO}_{2} / \mathrm{CH}_{3} \mathrm{NH}_{3} \mathrm{PbI}_{3} / \mathrm{HTM} / \mathrm{Au}$ & 0.99 & 20.70 & 59.9 & 12.23 & Duan et al. ${ }^{272}$ \\
\hline Sol-gel & $\begin{array}{l}\mathrm{FTO} / \mathrm{SnO}_{2} / \mathrm{CH}_{3} \mathrm{NH}_{3} \mathrm{PbI}_{3} / \\
\mathrm{SiGe} / \text { spiro-OMeTAD }\end{array}$ & $\begin{array}{l}\mathrm{CH}_{3} \mathrm{NH}_{3} \mathrm{PbI}_{3} / \mathrm{SiGe} / \text { spiro- } \\
\mathrm{OMeTAD}\end{array}$ & 1.05 & 22.8 & 66.2 & 15.8 & $\begin{array}{l}\text { Pinpithak } \\
\text { et al. }\end{array}$ \\
\hline Sol-gel & $\begin{array}{l}\mathrm{FTO} / \mathrm{SnO}_{2} \mathrm{ESL} / \\
\mathrm{CH}_{3} \mathrm{NH}_{3} \mathrm{PbI}_{3} / \mathrm{meso} / \mathrm{HTM} / \\
\mathrm{Ag}\end{array}$ & $\begin{array}{l}\mathrm{CH}_{3} \mathrm{NH}_{3} \mathrm{PbI}_{3} / \mathrm{meso} / \mathrm{HTM} / \\
\mathrm{Ag}\end{array}$ & 0.95 & 18.92 & 0.70 & 12.67 & Dong et al. ${ }^{274}$ \\
\hline Sol-gel & $\begin{array}{l}\mathrm{ITO} / \mathrm{SnO}_{2} /\left(\mathrm{FAPbI}_{3}\right)_{0.97^{-}} \\
\left(\mathrm{MAPbBr}_{3}\right)_{0.03} / \mathrm{spiro} / \mathrm{Au}\end{array}$ & $\begin{array}{l}\left(\mathrm{FAPbI}_{3}\right)_{0.97}-\left(\mathrm{MAPbBr}_{3}\right)_{0.03} / \\
\text { spiro/Au }\end{array}$ & 1.13 & 23.69 & 80.61 & 21.52 & Jiang et $a l^{275}$ \\
\hline Sol-gel & $\begin{array}{l}\mathrm{FTO} / \mathrm{SnO}_{2} /\left(\mathrm{FAPbI}_{3}\right)_{0.875^{-}} \\
(\mathrm{CsPbBr})_{0.125} / \mathrm{spiro}_{\mathrm{Au}}\end{array}$ & $\begin{array}{l}\left(\mathrm{FAPbI}_{3}\right)_{0.875^{-}} \\
\left(\mathrm{CsPbBr}_{3}\right)_{0.125} / \text { spiro/Au }\end{array}$ & 1.07 & 21.26 & 0.74 & 16.80 & Jung et $a l^{243}$ \\
\hline
\end{tabular}


Table 5 (Contd.)

\begin{tabular}{|c|c|c|c|c|c|c|c|}
\hline Method & Device layer $\left(\mathrm{SnO}_{2}\right)$ & $\begin{array}{l}\text { Doping/foreign element(s) } \\
\left(\mathrm{SnO}_{2}\right)\end{array}$ & $V_{\mathrm{OC}}(\mathrm{V})$ & $\begin{array}{l}J_{\mathrm{SC}}(\mathrm{mA} \\
\left.\mathrm{cm}^{-2}\right)\end{array}$ & FF (\%) & $\begin{array}{l}\text { PCE } \\
(\%)\end{array}$ & Reference \\
\hline Sol-gel & $\begin{array}{l}\mathrm{FTO} / \mathrm{SnO}_{2} \mathrm{QD} / \\
\mathrm{CH}_{3} \mathrm{NH}_{3} \mathrm{PbI}_{3} / \text { spiro- } \\
\text { OMeTAD/Au }\end{array}$ & $\begin{array}{l}\mathrm{QD} / \mathrm{CH}_{3} \mathrm{NH}_{3} \mathrm{PbI}_{3} / \text { spiro- } \\
\text { OMeTAD/Au }\end{array}$ & 1.11 & 20.94 & 0.73 & 16.97 & Yang et al. ${ }^{276}$ \\
\hline Spin-coating & 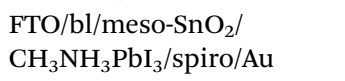 & $\mathrm{CH}_{3} \mathrm{NH}_{3} \mathrm{PbI}_{3} /$ spiro/Au & 1.11 & 22.80 & 75.78 & 19.21 & Xiong et al. ${ }^{277}$ \\
\hline Spin-coating & $\begin{array}{l}\mathrm{FTO} / \mathrm{m}-\mathrm{SnO}_{2} / \mathrm{CH}_{3} \mathrm{NH}_{3} \mathrm{PbI}_{3} / \\
\text { spiro-OMeTAD/Au }\end{array}$ & $\begin{array}{l}\mathrm{CH}_{3} \mathrm{NH}_{3} \mathrm{PbI}_{3} / \text { spiro- } \\
\text { OMeTAD/Au }\end{array}$ & 983 & 21.1 & 63 & 13.1 & $\begin{array}{l}\text { Roose } \\
\text { et } a .^{278}\end{array}$ \\
\hline Hydrothermal & $\begin{array}{l}\text { FTO/SnO }{ }_{2} \text { nanosheet } \\
\mathrm{CH}_{3} \mathrm{NH}_{3} \mathrm{PbI}_{3} / \text { spiro-/Au }\end{array}$ & $\mathrm{CH}_{3} \mathrm{NH}_{3} \mathrm{PbI}_{3} /$ spiro-/Au & 1.05 & 22.76 & 0.68 & 16.17 & Liu et $a .^{279}$ \\
\hline Sol-gel & $\begin{array}{l}\mathrm{FTO} / \mathrm{Nb}: \mathrm{SnO}_{2} / \\
\mathrm{CH}_{3} \mathrm{NH}_{3} \mathrm{PbI}_{3} / \text { spiro-/Au }\end{array}$ & $\mathrm{CH}_{3} \mathrm{NH}_{3} \mathrm{PbI}_{3} /$ spiro-/Au & 1.08 & 22.36 & 0.72 & 17.57 & Ren et $a l .{ }^{280}$ \\
\hline Hydrothermal & $\begin{array}{l}\text { FTO/SnO }{ }_{2} \text { nanosheet } / \mathrm{C}_{60} / \\
\mathrm{CH}_{3} \mathrm{NH}_{3} \mathrm{PbI}_{3} / \text { spiro-/Au }\end{array}$ & $\mathrm{C}_{60} / \mathrm{CH}_{3} \mathrm{NH}_{3} \mathrm{PbI}_{3} /$ spiro-/Au & 1.03 & 23.62 & 75 & 18.31 & Wu et al..$^{281}$ \\
\hline $\begin{array}{l}\text { Atomic layer } \\
\text { deposition }\end{array}$ & $\begin{array}{l}\mathrm{FTO} / \mathrm{SnO}_{2} / \mathrm{CH}_{3} \mathrm{NH}_{3} \mathrm{PbI}_{3} / \\
\mathrm{HTL} / \mathrm{Au}\end{array}$ & $\mathrm{CH}_{3} \mathrm{NH}_{3} \mathrm{PbI}_{3} / \mathrm{HTL} / \mathrm{Au}$ & 1.14 & 21.3 & 74 & 18.4 & $\begin{array}{l}\text { Baena } \\
\text { et } a l^{234}\end{array}$ \\
\hline $\begin{array}{l}\text { Pulse layer } \\
\text { deposition }\end{array}$ & $\begin{array}{l}\mathrm{FTO} / \mathrm{SnO}_{2} / \mathrm{PCBM} / \\
\mathrm{CH}_{3} \mathrm{NH}_{3} \mathrm{PbI}_{3} / \text { spiro-/Au }\end{array}$ & $\begin{array}{l}\mathrm{PCBM} / \mathrm{CH}_{3} \mathrm{NH}_{3} \mathrm{PbI}_{3} / \text { spiro-/ } \\
\mathrm{Au}\end{array}$ & 1.11 & 21.6 & 71 & 17.03 & Chen et $a l^{282}$ \\
\hline $\begin{array}{l}\text { Chemical bath } \\
\text { deposition }\end{array}$ & $\begin{array}{l}\mathrm{FTO} / \mathrm{SnO}_{2} / \mathrm{CH}_{3} \mathrm{NH}_{3} \mathrm{PbI}_{3} / \\
\text { spiro-OMeTAD/Au }\end{array}$ & $\begin{array}{l}\mathrm{CH}_{3} \mathrm{NH}_{3} \mathrm{PbI}_{3} / \text { spiro- } \\
\text { OMeTAD/Au }\end{array}$ & 1.13 & 22.95 & 79 & 20.56 & $\mathrm{Bu}$ et $a l^{283}$ \\
\hline Hydrothermal & HT/FTO-SnO 2 & HT/FTO & 1.08 & 21.35 & 74.89 & 17.37 & Liu et $a .^{252}$ \\
\hline Spin-coating & $\begin{array}{l}\mathrm{ITO} / \mathrm{SnO}_{2} / \mathrm{ZTO} / \\
\mathrm{CH}_{3} \mathrm{NH}_{3} \mathrm{PbI}_{3} / \text { spiro- } \\
\text { OMeTAD/Au }\end{array}$ & $\begin{array}{l}\mathrm{ZTO} / \mathrm{CH}_{3} \mathrm{NH}_{3} \mathrm{PbI}_{3} / \text { spiro- } \\
\text { OMeTAD } / \mathrm{Au}\end{array}$ & 1.13 & 22.6 & 80.2 & 20.5 & Guo et $a .^{284}$ \\
\hline Spin-coating & $\begin{array}{l}\text { FTO/SnO } \\
2 / \mathrm{MAPbI}_{3} / \text { spiro- }^{-} \\
\text {OMeTAD/Au }\end{array}$ & $\mathrm{MAPbI}_{3} /$ spiro-OMeTAD/Au & 1.11 & 21.44 & 74.58 & 17.83 & $\begin{array}{l}\text { Zhang } \\
\text { et al. }\end{array}$ \\
\hline Sol-gel & NPT-SnO ${ }_{2}$ & NPT & 1.12 & 21.82 & 0.83 & 20.3 & $\begin{array}{l}\text { Subbiah } \\
\text { et al. }\end{array}$ \\
\hline Sputtering & $\begin{array}{l}\mathrm{FTO} / \mathrm{SnO}_{2} / \mathrm{CH}_{3} \mathrm{NH}_{3} \mathrm{PbI}_{3} / \\
\text { spiro-OMeTAD/Au }\end{array}$ & $\begin{array}{l}\mathrm{CH}_{3} \mathrm{NH}_{3} \mathrm{PbI}_{3} / \text { spiro- } \\
\text { OMeTAD/Au }\end{array}$ & 1.08 & 23.7 & 0.79 & 20.3 & Qiu et $a l . .^{287}$ \\
\hline $\begin{array}{l}\text { Plasma } \\
\text { treatment }\end{array}$ & $\begin{array}{l}\text { FTO/c-SnO } \\
\mathrm{CH}_{3} \mathrm{NH}_{3} \mathrm{PbI}_{3} / \text { spiro- } \\
\text { OMeTAD/Au }\end{array}$ & $\begin{array}{l}\mathrm{MAPI} / \mathrm{CH}_{3} \mathrm{NH}_{3} \mathrm{PbI}_{3} / \text { spiro- }^{-} \\
\text {OMeTAD/Au }\end{array}$ & 108 & 20.4 & 76.3 & 19.4 & $\begin{array}{l}\text { Méndez } \\
\text { et } a \text {. }^{288}\end{array}$ \\
\hline
\end{tabular}

${ }^{a}$ Abbreviations: $\mathrm{ITO}=$ indium tin oxide, $\mathrm{FTO}=$ fluorine doped tin oxide, $\mathrm{ZTO}=$ zinc tin oxide, $\mathrm{CNT}=$ carbon nanotube, $\mathrm{QD}=\mathrm{quantum}$ dot, $\mathrm{HT}$ $\mathrm{SnO}_{2}=$ high temperature tin oxide, $\mathrm{LT} \mathrm{SnO}_{2}=$ low temperature tin oxide, $\mathrm{PEN}-\mathrm{Br}=\operatorname{poly}\left(9,9-\mathrm{bis}\left(3^{\prime}(N, N\right.\right.$-dimethyl)- $N$-ethylammonium-propyl-2,7fluorene)-alt-2,7-(9,9-dioctylfluorene))dibromide, spiro-OMeTAD $=2,2^{\prime}, 7,7^{\prime}$-tetrakis $\left[N, N\right.$-di(4-methoxyphenyl)amino]-9,9' $9^{\prime}$-spirobifluorene, PET $=$ polyethylene terephthalate, $\mathrm{SnO}_{2}$ ESL $=$ tin oxide electron selective layer, $\mathrm{HTM}=$ high temperature mesoporous, $\mathrm{FAPbI}_{3}=$ single crystal formamidinium lead iodide, $\mathrm{MAPbI}_{3}$ (or) MAPI = methylammonium lead halide, PCBM = phenyl C-61 butyric acid methyl ester, NPT = nitrogen plasma treated, $\mathrm{HTL}=$ hole transporting layer.

particular to $\mathrm{SnO}_{2}$ surfaces. Since then, there have been a plethora of reports on plasma engineered $\mathrm{SnO}_{2}$ nanostructures for a wide range of applications, e.g. photovoltaics, sensors and catalysis. ${ }^{57,85,295}$

$\mathrm{SnO}_{2}$ thin films are typically used as a TCO for the front electrode in silicon thin-film solar cells, due to their wide bandgap $(\sim 3.6 \mathrm{eV})$, transparency in the visible region and high electrical conductivity. Hydrogen plasma is commonly used to prepare hydrogenated amorphous and microcrystalline silicon films. ${ }^{296}$ Towards this end, Kim et al. studied the effects of hydrogen plasma treatment on the structural and electrical properties of sputtered $\mathrm{SnO}_{2}$ thin films. ${ }^{186}$ They found that hydrogen plasma treatment degraded the crystalline quality and optical transmittance and cause etching of the $\mathrm{SnO}_{2}$ films. Interestingly, hydrogen plasma treatment led to improved electrical conductivity along with the increase in carrier concentration, and this was attributed to the formation of oxygen vacancies in undoped $\mathrm{SnO}_{2}$ films. Tang et al. were able to modulate the electronic properties, surface energy, and roughness of fluorine-doped $\mathrm{SnO}_{2}$ films using oxygen plasma treatment. ${ }^{297}$ Both the work function and hydrophilicity of $\mathrm{SnO}_{2}$ were found to increase after oxygen plasma treatment. In a unique approach, Chen and Thimsen were able to synthesize highly conductive ATO nanocrystals using dual-nonthermal plasmas. ${ }^{112}$ They were able to achieve a high conductivity of $0.1 \mathrm{~S} \mathrm{~cm}^{-1}$ for the as-deposited porous ATO films prepared using this approach. During the synthesis, $\mathrm{Sn}$ and $\mathrm{Sb}$ vapor precursors were fed into a dual-zone (discharge) flow-through plasma reactor. The reagent passed through the first plasma zone to form metallic Sb-Sn alloy NPs. From the first discharge (zone 1), the alloy NPs followed the carrier gas through to the second discharge (zone 2) containing $\mathrm{O}_{2}$ to become oxidized. The ATO nanocrystals were then deposited onto various substrates (silicon wafer, glass, fused silica, or single crystal $\mathrm{NaCl}$ ). The as-deposited films showed a high optical transmittance of $>90 \%$ over the entire visible wavelength regime. Hence, ex situ plasma treatment could be used to modify the surface composition, energy band structure, and surface energy 
to facilitate efficient carrier transport at the FTO interfaces, eventually improving the device performance.

Like silicon solar cells, $\mathrm{SnO}_{2}$ plays a critical role in PSCs as well. ${ }^{298}$ The high dopant concentration $\left(N_{\mathrm{d}}=10^{20} \mathrm{~cm}^{-3}\right)$ in FTO results in the formation of a very thin space charge region at the interface. The decrease in the width of the space charge region increases the probability of electrons tunneling across, resulting in a reduced performance. To overcome this issue, it is customary to introduce a thin layer of the electron blocking layer (metal oxide) in the form of $\mathrm{TiO}_{2}$ or $\mathrm{SnO}_{2}$ atop FTO. However, the growth or deposition of this electron blocking layer faces several challenges such as the formation of pinholes and uniform thickness. To overcome this, Dao et al. developed a new strategy to increase the uniformity and reduce the pinholes in the $\mathrm{TiO}_{2}$ layer by atmospheric pressure plasma treatment of FTO. ${ }^{299}$ They observed a significant increase in the efficiency of the PSC where the $\mathrm{TiO}_{2}$ blocking layer was deposited on plasma-treated FTO. Argon plasma $(150 \mathrm{~W})$ treatment for 1 minute transformed the FTO surface superhydrophilic. The increase in the efficiency was attributed to the suppression of electron recombination at the FTO interfaces due to the highdensity, uniform, and pinhole-free $\mathrm{TiO}_{2}$ layer on the surface of the plasma-treated FTO.

In another interesting work, Wang et al. demonstrated that a low temperature processed $\mathrm{SnO}_{2}$ could be an excellent material for the electron selective layer (ESL) material. ${ }^{300}$ Through plasma-enhanced atomic layer deposition (PEALD) they could deposit $\mathrm{SnO}_{2}$ at temperatures less than $100{ }^{\circ} \mathrm{C}$, which is significant, considering the compatibility with large-scale rollto-roll manufacturing and other potential opportunities for flexible solar cells. The as-fabricated PSCs with $\mathrm{SnO}_{2}$ deposited through PEALD and passivated with a $\mathrm{C}_{60}$-self-assembled monolayer exhibited maximum PCEs of $19.03 \%$ and $16.80 \%$ under reverse voltage on glass and flexible polymer substrates, respectively, as shown in Fig. 20. In the same way, Kuang et al. also used PEALD for $\mathrm{SnO}_{2}$ in PSCs. ${ }^{301}$ They studied the influence of deposition temperature on the material properties of $\mathrm{SnO}_{2}$. Highly transparent amorphous $\mathrm{SnO}_{2}$ films were prepared in a temperature range of $50-200{ }^{\circ} \mathrm{C}$. The film deposited at $200{ }^{\circ} \mathrm{C}$ showed the best band alignment at the $\mathrm{SnO}_{2}$ :perovskite interface, while the film deposited at $50{ }^{\circ} \mathrm{C}$ showed a considerable band offset. However, for a $15 \mathrm{~nm}$ thick layer, this band offset did not affect the electron transport at the interface and the PSCs with either 50 or $200{ }^{\circ} \mathrm{C} \mathrm{SnO}_{2}$ ETL demonstrated comparable initial PCEs. The $\mathrm{SnO}_{2}$ films fabricated at $50{ }^{\circ} \mathrm{C}$ were found to be resistive, while the $200{ }^{\circ} \mathrm{C} \mathrm{SnO}_{2}$ films showed a low electrical resistivity of $1.8 \times 10^{-3} \Omega \mathrm{cm}$ and high carrier density and mobility of $9.6 \times 10^{19} \mathrm{~cm}^{-3}$ and $36.0 \mathrm{~cm}^{2} \mathrm{~V}^{-1} \mathrm{~s}^{-1}$, respectively. As a result, the PSCs with $200{ }^{\circ} \mathrm{C} \mathrm{SnO}_{2}$ retain their initial performance at the maximum power point for over $16 \mathrm{~h}$. Thus, the reports by Wang et al. and Kuang et al. envisage the prospect of integrating PEALD for roll-to-roll applications. ${ }^{300,301}$

$\mathrm{Hu}$ et al. used $\mathrm{SnO}_{x}$ grown by ALD as an electron extraction layer to design ITO-free PSCs. ${ }^{302}$ They replaced ITO with a semitransparent $\mathrm{SnO}_{x} / \mathrm{Ag}$ electrode, which simultaneously served as an electron extraction layer delivering a PCE of $11 \%$. They thoroughly investigated the electronic properties of $\mathrm{SnO}_{x}$ grown by ALD using $\mathrm{H}_{2} \mathrm{O}$, ozone, or oxygen plasma as the oxidant. Though there were no obvious differences in the crystal structure, morphology, or surface energy between the $\mathrm{SnO}_{x}$
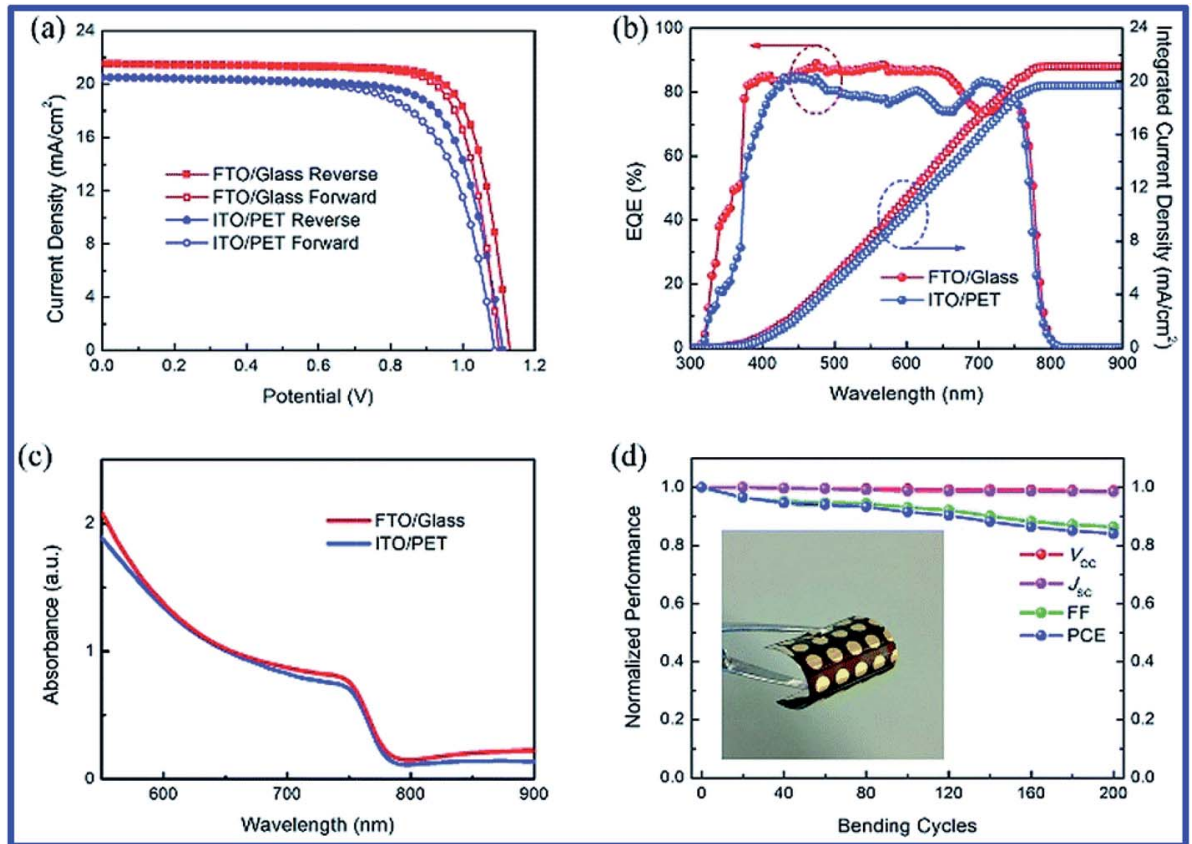

Fig. 20 The best-performing devices based on PEALD SnO 2 coated FTO/glass and ITO/PET substrates: (a) J-V curves under reverse and forward voltage scans. (b) EQE spectra and their corresponding integrated photocurrents. (c) Absorption spectra of perovskite films. (d) Normalized performance parameters of a flexible PVSC versus bending cycles. Photograph of a flexible device after the bending test (inset), ${ }^{300}$ presented with permission and copyright. 


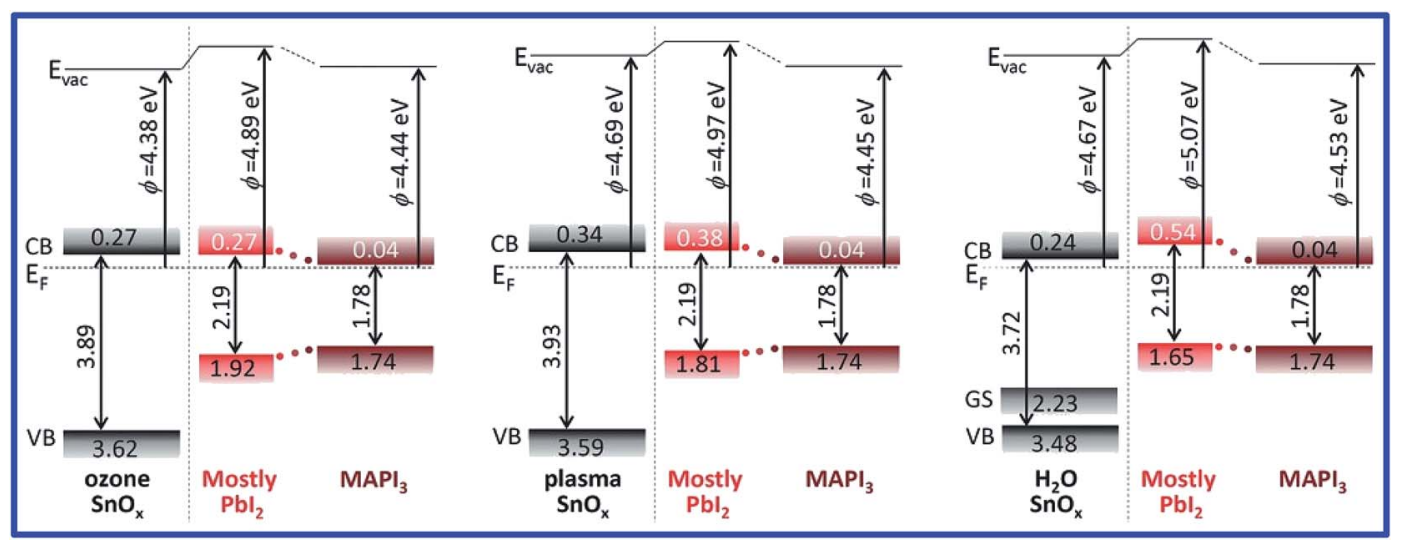

Fig. 21 Electronic structure of different $\mathrm{SnO}_{x}$ variants in their pristine state and of a thin and thick (bulk-like) MAPbl 3 layer deposited on top of them. The energy level positions were determined by UPS and IPES measurements. Abbreviations $-E_{\text {vac: }}$ : vacuum level; EF: Fermi energy; and $\varphi$ : work function. The values written at the VB and the CB mark their distance from the Fermi energy, ${ }^{302}$ presented with permission and copyright.

samples, the PSCs showed different performances. However, work function measurements using Kelvin probe microscopy and ultraviolet photoelectron spectroscopy (UPS) on these $\mathrm{SnO}_{x}$ layers showed a trend of plasma-SnO $\mathrm{S}_{x}>\mathrm{H}_{2} \mathrm{O}-\mathrm{SnO}_{x}>$ ozone$\mathrm{SnO}_{x}$. Interestingly, only for $\mathrm{H}_{2} \mathrm{O}-\mathrm{SnO}_{x}$, an oxygen vacancy induced gap state (GS) at about $2.23 \mathrm{eV}$ below the Fermi level was observed only for $\mathrm{H}_{2} \mathrm{O}-\mathrm{SnO}_{x}$. They also found that there is always formation of the $\mathrm{PbI}_{2}$ interfacial layer with an electronic bandgap of $2.19 \mathrm{eV}$ between $\mathrm{MAPbI}_{3}$ and $\mathrm{SnO}_{x}$. Fig. 21 shows the interfacial electronic band alignment for three $\mathrm{SnO}_{x}$ electrodes. Based on their measurements and performance, they concluded that ozone- $\mathrm{SnO}_{x}$ was the best hole blocking layer and designed an ITO-free semitransparent bottom electrode based on $\mathrm{SnO}_{x}$ / $\mathrm{Ag} / \mathrm{SnO}_{x}$ architecture. Here, $\mathrm{H}_{2} \mathrm{O}-\mathrm{SnO}_{x}$ acted as the permeation barrier to protect the $\mathrm{Ag}$ from corrosion.

Although ALD and sputtering techniques can produce uniform and crystalline thin films, their integration onto large scale production and roll-to-roll fabrication routes is a challenge. From a practical point of view, it is important to simplify the entire fabrication process to address these challenges. For this purpose, $\mathrm{Yu}$ et al. ${ }^{303}$ devised a superfast $(<5 \mathrm{~min})$ facile route to synthesize $\mathrm{SnO}_{2}$ at room-temperature $\left(<50{ }^{\circ} \mathrm{C}\right)$ using atmospheric $\mathrm{Ar} / \mathrm{O}_{2}$ plasma, as shown in Fig. 22a. Compared with thermally annealed $\mathrm{SnO}_{2}\left(\mathrm{~T}-\mathrm{SnO}_{2}\right)$ thin films, the plasma synthesized $\mathrm{SnO}_{2}$ films exhibited superior properties viz. higher electrical conductivity, better electron mobility, and low density of charge trap sites. Consequently, plasma synthesized $\mathrm{SnO}_{2}$ based PSCs achieved a superior PCE of 19.56\%. The comparatively high PCE was attributed to efficient electron extraction and reduced nonradiative recombination at the plasma synthesized-SnO $\mathrm{SHL}_{2} \mathrm{ET} /$ perovskite interface, which relaxed the hysteresis and improved the resistance of PSCs to sunlight.

Another approach to improve the efficiency of solutionprocessed PSCs was proposed by Subbiah et $a .^{286}$ This involved the use of a low-power $\mathrm{N}_{2}$ plasma to form compact $\mathrm{SnO}_{2}$ from a spin-coated $\mathrm{SnCl}_{4} \cdot 5 \mathrm{H}_{2} \mathrm{O}$ solution, as schematically shown in Fig. 22b. Most importantly, this technique could be applied to fabricate PSCs on rigid as well as on flexible substrates. When compared with $\mathrm{O}_{2}$ plasma treatment, $\mathrm{SnO}_{2}$ film formation from the $\mathrm{Sn}$ metal halide precursor materialized only in the presence of a $\mathrm{N}_{2}$ plasma. This was attributed to the deep UV emission lines ( $c a .290-390 \mathrm{~nm}$ ) of $\mathrm{N}_{2}$ plasma alongside the NO* species, which provide the necessary energy to initiate the cleaving of metal alkoxy bonds. To prove the effectiveness of the process, they compared the PSC performance composed of either $\mathrm{N}_{2}$ plasma-treated $\left(\mathrm{NPT}-\mathrm{SnO}_{2}\right)$ or thermally oxidized ( $\mathrm{TA}-\mathrm{SnO}_{2}$ ) films. The champion device with $\mathrm{NPT}-\mathrm{SnO}_{2}$ as the ETL witnessed a PCE of $20.3 \%$, a $V_{\text {OC }}$ of $\sim 1.12 \mathrm{~V}$, an FF of $\sim 0.83$, and a $J_{\mathrm{SC}}$ of $\sim 21.82 \mathrm{~mA} \mathrm{~cm}{ }^{-2}$ under the reverse scan, whereas the $\mathrm{TA}^{-} \mathrm{SnO}_{2}$-based champion device demonstrated a PCE of $19.9 \%$, a $V_{\mathrm{OC}}$ of $\sim 1.08 \mathrm{~V}$, an FF of $\sim 0.81$, and a $J_{\mathrm{SC}}$ of $\sim 22.9 \mathrm{~mA} \mathrm{~cm}{ }^{-2}$. The flexible device fabricated with $\mathrm{NPT}-\mathrm{SnO}_{2}$ showed a best PCE of $18.1 \%$ under a reverse scan, with the corresponding performance parameters of $V_{\mathrm{OC}} \sim 1.05 \mathrm{~V}, \mathrm{FF} \sim$ $0.76, J_{\mathrm{SC}} \sim 22.8 \mathrm{~mA} \mathrm{~cm}{ }^{-2}$ and a stable PCE of $17.1 \%$. It is to be noted that a layer of mesoporous alumina $\left(\mathrm{Al}_{2} \mathrm{O}_{3}\right)$ was introduced on top of NPT-SnO ${ }_{2}$ to compensate for the presence of pinholes. Importantly, the device retained $90 \%$ of the initial PCE after 1000 cycles of bending. Details related to the flexible device are presented in Fig. 23.

Recently, Smith et al. studied the post-processing effects of spray-coated $\mathrm{SnO}_{2}$ films by UV ozone or $\mathrm{O}_{2}$ plasma on the device performance. ${ }^{304}$ The device with UV ozone treated ETL outperformed the one with the $\mathrm{O}_{2}$ plasma-treated ETL. The $\mathrm{O}_{2}$ plasma apparently makes the surface Sn-rich and O-poor. It was also found that plasma treatment significantly downshifted the Fermi level, resulting in a loss of electron selectivity along with a drop in $V_{\mathrm{OC}}$ and an increase in $J-V$ hysteresis. Hence, in some cases, $\mathrm{O}_{2}$ plasma can have a detrimental impact on the $\mathrm{SnO}_{2} /$ perovskite interface and device performance. To gain a better understanding, Luan et al. were able to increase the PCE by employing $\mathrm{O}_{2}$ plasma treatment to treat 2,2,2-trifluoroethanol (TFE)-incorporated $\mathrm{SnO}_{2}$ ETL films. ${ }^{244}$ The presence of a strong electron-withdrawing group (trifluoromethyl) improved the electron mobility in the $\mathrm{SnO}_{2}$ ETL. They found that by carefully controlling the plasma power to $60 \mathrm{~W}$ used for treating $\mathrm{SnO}_{2}$, 


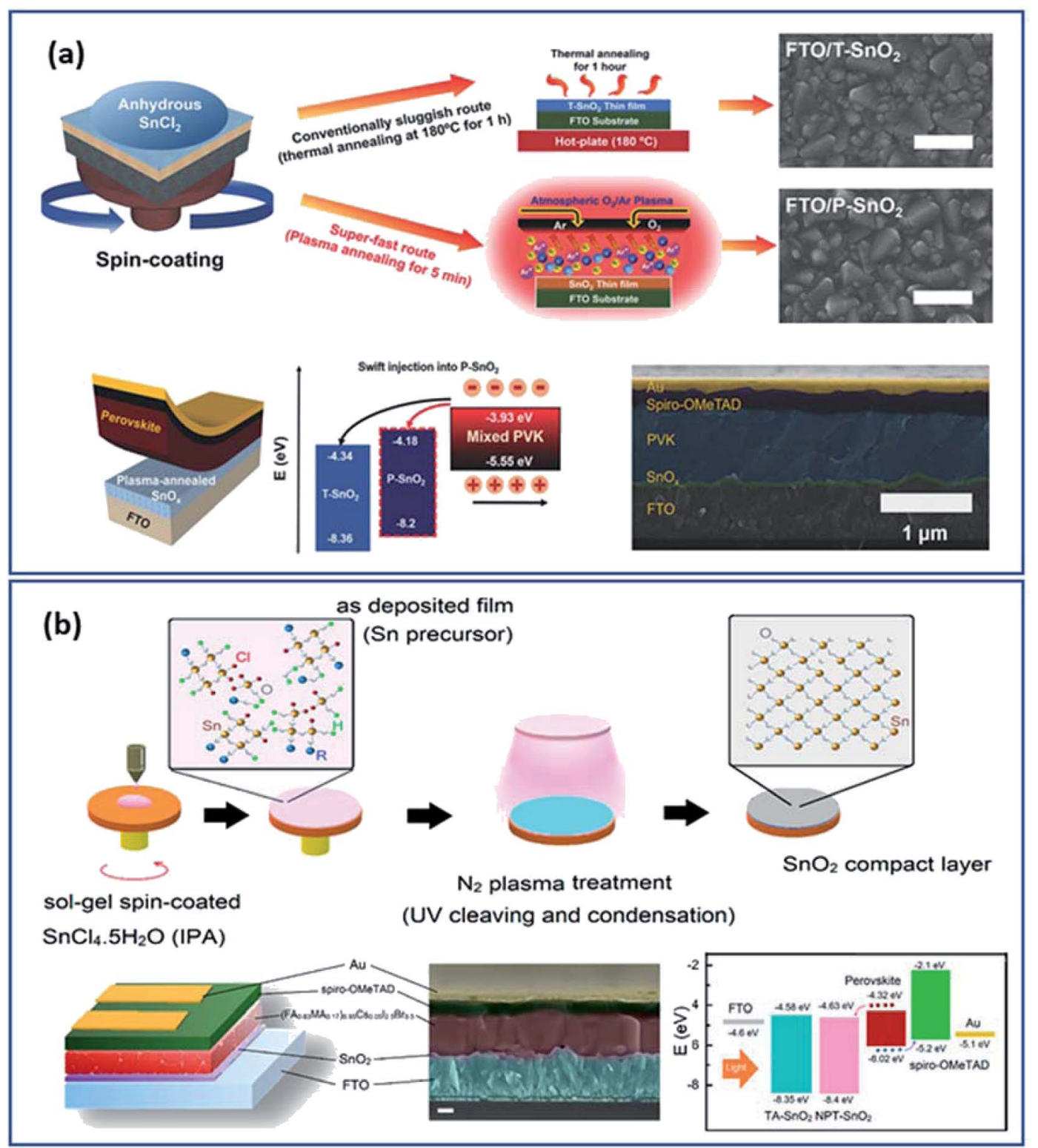

Fig. 22 Schematic of $\mathrm{SnO}_{2}$ thin film fabrication by (a) Yu et al. using thermal or $\mathrm{O}_{2} / \mathrm{Ar}$ plasma with corresponding field-emission scanning electron microscopy (FE-SEM) images. $\mathrm{T}-\mathrm{SnO}_{2}$ was annealed at $180^{\circ} \mathrm{C}$ for $60 \mathrm{~min}$, and $\mathrm{P}-\mathrm{SnO}_{2}$ was developed by plasma energy with gas flow rates of $0.008 \mathrm{~L} \mathrm{~h}^{-1}(\mathrm{Ar})$ and $0.06 \mathrm{~L} \mathrm{~h}^{-1}\left(\mathrm{O}_{2}\right)$ for $5 \mathrm{~min}^{303}$ (b) Subbiah et al. using a modified sol-gel technique by employing low-power RF $\mathrm{N}_{2}$ plasma exposure for $60 \mathrm{~min}$ in an approximate pressure of 1 Torr, ${ }^{286}$ presented with permission and copyright.

the PCE could be increased from $20.92 \%$ to $21.68 \%$ ( $V_{\text {OC }}: 1.12 \mathrm{~V}$, $J_{\mathrm{SC}}: 24.06 \mathrm{~mA} \mathrm{~cm}^{-2}$ and FF: 0.802). However, at higher power $(140 \mathrm{~W}), V_{\text {OC }}$ dropped from $1.12 \mathrm{~V}$ to $1.07 \mathrm{~V}$ due to the increase in the work function (from 4.21 to $4.40 \mathrm{eV}$ ) away from the conduction band of the perovskite film $(4.18 \mathrm{eV})$ similar to the findings of Smith et al. ${ }^{304}$

A significant amount of research has been conducted on graphenes during the last decade. This is driven by the realization of the immense capabilities possessed by this wonder material. Applications are already recognized in a wide range of disciplines from ultra-fast and flexible electronics to optoelectronic devices, supercapacitors, water remediation, DNA attachment, photocatalysis, oxygen reduction reaction catalysts, and many more. To realize these pathways, functionalization of graphene and its oxide played a crucial role. Many techniques of functionalization have been applied to extend the application prospects of graphene. ${ }^{\mathbf{1 4 2}}$

In the present review, we discuss the plasma engineering of oxide surfaces as a potential alternative to conventional techniques. Plasma functionalization is advantageous in terms of controllability and selectivity associated with it. The ionic species present in plasma can tune the electronic and optical properties of oxides and can even control the surface energy and processes. Wet chemical functionalization involving precursors and by-products fails in achieving localized effects in oxide semiconductors for PV applications. With plasma 

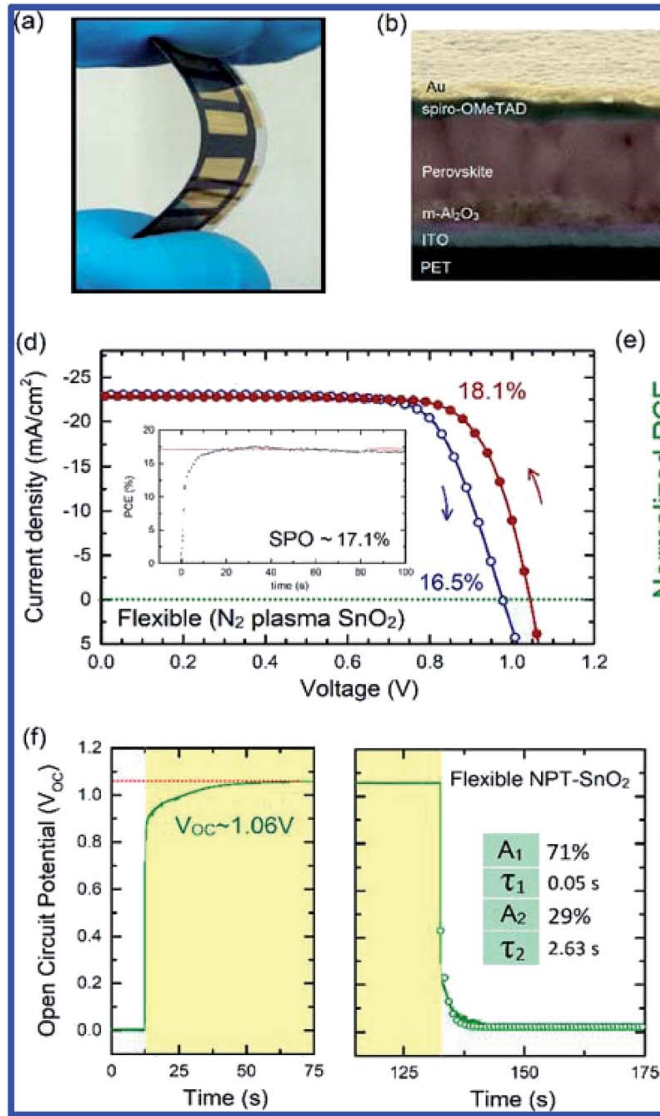

(b)

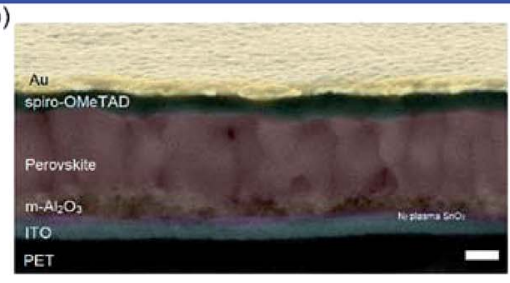

(c)

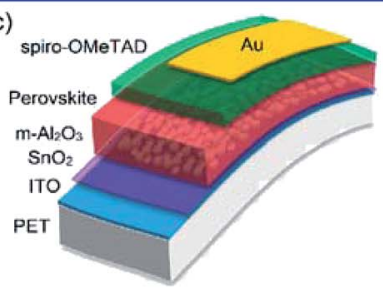

(e)

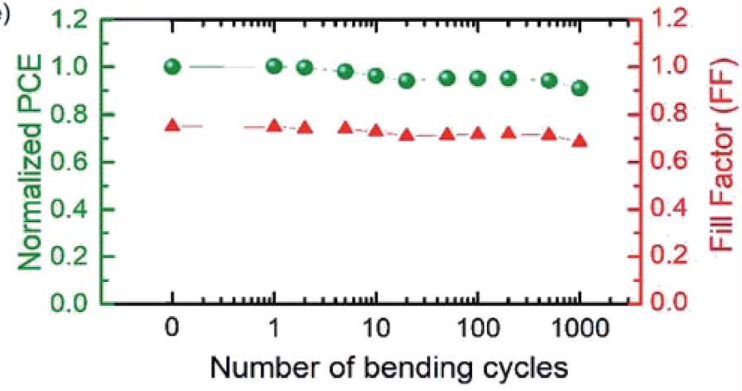

(g)

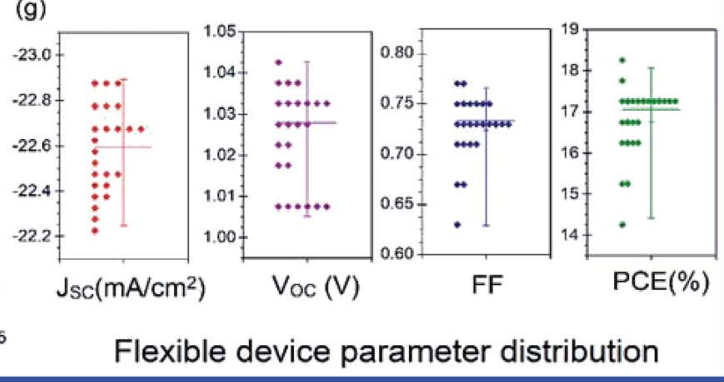

Number of bending cycles

Fig. 23 (a) Photograph depicting the flexible nature of the devices fabricated using room-temperature NPT-SnO 2 ETL. (b) SEM cross-sectional image of a flexible device. (c) Device architecture schematic of the flexible device fabricated by Subbiah et al. ${ }^{286}$ (d) Champion light $\mathrm{J}-V$ characteristics of the flexible device with a PCE of $18.1 \%$ and a stable power output efficiency of $17.1 \%$ under 1 sun illumination. (e) Normalized device efficiency and fill factor of the flexible device being subjected to 1000 bending cycles. (f) Transient $V_{\text {Oc }}$ measurements in the seconds scale to illustrate the associated rise and decay constants. (g) Histogram of the various device parameters extracted from a batch of 24 flexible devices. All efficiencies were measured under the reverse scan, ${ }^{286}$ presented with permission and copyright.

functionalization, it is possible to precisely tailor-design oxide properties by inducing localized charges. The advantages of this technique include time and cost-effectiveness. This process is environmentally friendly and opens up future pathways for large-scale industrial implementation. However, most of the plasma processes involve low-pressure vacuum-based systems. Atmospheric pressure plasma can be a promising candidate for various plasma-based functionalization applications. In atmospheric pressure plasma jet (APJJ), the plasma is not confined within the dimensions of the electrodes. Nevertheless, it is important to understand the characteristics of plasma being used, to make the process beneficial.

\subsection{Thermally treated $\mathrm{SnO}_{2}$ for PSCs}

For $\mathrm{SnO}_{2}$ thin films, the effect of the annealing temperature on the film properties is a key parameter as it has influence on its electrical properties (resistivity, carrier concentration and mobility, and conductive type), surface morphology, and crystal quality of the films. Optimization of parameters is essential to fabricate efficient devices. Normally, $\mathrm{SnO}_{2}$ films treated at higher temperatures will exhibit better crystallinity, which improves the charge carrier and the optical transmittance. ${ }^{57,305}$ Yuan et al. demonstrated the influence of the thermal annealing temperature on the electrical and optical properties of $\mathrm{SnO}_{2}$ thin films deposited on glass substrates by spin-coating. ${ }^{306} \mathrm{SnO}_{2}$ films annealed in air show an increase in the optical transmittance by increasing the annealing temperature from 100 to $300{ }^{\circ} \mathrm{C}$, but the transmittance decreased upon further increasing the temperature to $500{ }^{\circ} \mathrm{C}$. Particles in the film annealed at $100{ }^{\circ} \mathrm{C}$ show a high surface roughness. When the temperature was increased to $300{ }^{\circ} \mathrm{C}$, the surface roughness of the films decreased due to the increase in the surface diffusion of atoms with higher kinetic energy. At $500{ }^{\circ} \mathrm{C}$, cracks and blisters occurred due to the rapid growth of the particles, resulting in an increase in surface roughness. As a result, the transmittance of $\mathrm{SnO}_{2}$ thin films decreased at $500{ }^{\circ} \mathrm{C}$. An annealing temperature below $500{ }^{\circ} \mathrm{C}$ has a positive effect on the sheet resistance and transmittance in the visible light region of $\mathrm{SnO}_{2}$ thin films.

Generally, for the fabrication of high-efficiency solar cells, metal-oxide electron selective layers (ESLs) annealed at high temperatures (HT) are preferred over those at low-temperatures (LT), due to the higher carrier mobility. ${ }^{57}$ However, as 
demonstrated by Ke et al., for the $\mathrm{SnO}_{2}$ ESLs, the situation is quite different. ${ }^{268}$ To compare the efficiency of low- and hightemperature annealed $\mathrm{SnO}_{2}$ on the performance of perovskite solar cells (PSCs), the authors prepared $\mathrm{SnO}_{2}$ films over FTO substrates by spin coating of the $\mathrm{SnCl}_{2} \cdot 2 \mathrm{H}_{2} \mathrm{O}$ precursor, followed by thermal annealing in air at $185{ }^{\circ} \mathrm{C}\left(\mathrm{LT}-\mathrm{SnO}_{2}\right)$ and at $500{ }^{\circ} \mathrm{C}\left(\mathrm{HT}^{-\mathrm{SnO}_{2}}\right)$ for $1 \mathrm{~h}$. Perovskite solar cells using $\mathrm{LT}-\mathrm{SnO}_{2}$ ESLs exhibited a better performance than the cells using HT$\mathrm{SnO}_{2}$, due to a better film coverage, lower electron concentration, higher electron mobility, and a wider band gap. The use of LT annealing of $\mathrm{SnO}_{2}$ ESL is an important advantage due to its low cost, easy preparation, and better performance in PSCs.

Another way to improve the photovoltaic performance by boosting the electrical conductivity of $\mathrm{SnO}_{2}$ ESLs is using thermal annealing to reduce or even eliminate the current density-voltage $(J-V)$ hysteresis. Jung et al. used tin(Iv) isopropoxide as the precursor to deposit $\mathrm{SnO}_{2}$ ETL films onto the FTO glass substrate. ${ }^{243}$ The films were annealed in ambient air at $100,150,200,250,300,350,400$ and $500{ }^{\circ} \mathrm{C}$ for $30 \mathrm{~min}$. The results showed that the $J-V$ curves and the photovoltaic performance depend on the annealing temperature. The difference between the reverse and forward scanned $J-V$ curves decreased as the temperature was increased from $150{ }^{\circ} \mathrm{C}$ to $250{ }^{\circ} \mathrm{C}$, which minimized at $250{ }^{\circ} \mathrm{C}$ and then increased again as the temperature was increased from $300{ }^{\circ} \mathrm{C}$ to $500{ }^{\circ} \mathrm{C}$. This behaviour directly affected the power conversion efficiency (PCE), which reached a maximum average value of $16.08 \%$ at $250{ }^{\circ} \mathrm{C}$. Similar results were also reported by Wang et al. ${ }^{307}$ The photovoltaic performance of PEALD $\mathrm{SnO}_{2}$ ESLs was improved by low-temperature thermal annealing in ambient air due to a reduction in the imbalance of charge transportation and consequently to a significant reduction in the degrees of $J-V$ hysteresis.

The change in the annealing temperature is also capable of modifying the conducting type of transparent conductive $\mathrm{SnO}_{2}: \mathrm{Zn}$ thin films, as shown in Ni et al. ${ }^{201}$ Based on the Hall measurements, it can be confirmed that the $\mathrm{SnO}_{2}: \mathrm{Zn}$ films annealed below $400{ }^{\circ} \mathrm{C}$ possessed an n-type behaviour. Upon increasing the annealing temperature within the range of 400$500{ }^{\circ} \mathrm{C}$, the films showed a p-type conductivity, but at $500{ }^{\circ} \mathrm{C}$ an increase in resistivity and a decrease in carrier concentration were also observed. Finally, for the films annealed above $500{ }^{\circ} \mathrm{C}$, a conversion in conductivity from p-type only to both p-type and n-type as the major and secondary conduction type occurred. This behaviour might be attributed to the inability of $\mathrm{Zn}$ atoms to substitute Sn at lower temperatures due to a lack of activation energy. When the annealing temperature increases, the acceptor effect of $\mathrm{Zn}$ substituting $\mathrm{Sn}$ is activated, resulting in $\mathrm{p}$ type films.

Several studies indicate that a strong correlation exists between the characteristics of the $\mathrm{SnO}_{2}$ layer and the device performance, which is strictly connected to surface chemistry. For example, Wang et al. ${ }^{308}$ demonstrated a mesoporous $\mathrm{SnO}_{2}$ $\left(\mathrm{m}-\mathrm{SnO}_{2}\right)$ layer with different concentrations of polyethylene glycol (PEG) (0, 3, 6, 9, and 12\%). The colloidal $\mathrm{SnO}_{2}$ precursor solution was spin coated (4000 rpm, $20 \mathrm{~s}$ ) on the ITO substrate with and without PEG and then annealed at $180{ }^{\circ} \mathrm{C}$ for $20 \mathrm{~min}$. The device exhibits excellent performance with a PCE of $20.82 \%$, a $V_{\text {OC }}$ of $1.10 \mathrm{~V}$, a $J_{\mathrm{SC}}$ of $24.56 \mathrm{~mA} \mathrm{~cm}^{-2}$, and an $\mathrm{FF}$ of $77.10 \% .{ }^{308}$ Kuang et al. fabricated a $\mathrm{SnO}_{2}$ based device (glass/ ITO/ALD $\mathrm{SnO}_{2} / \mathrm{CH}_{3} \mathrm{NH}_{3} \mathrm{PbI}_{3} /$ spiro-OMeTAD/Au) with different annealing temperatures. The solar cell exhibited a PCE of
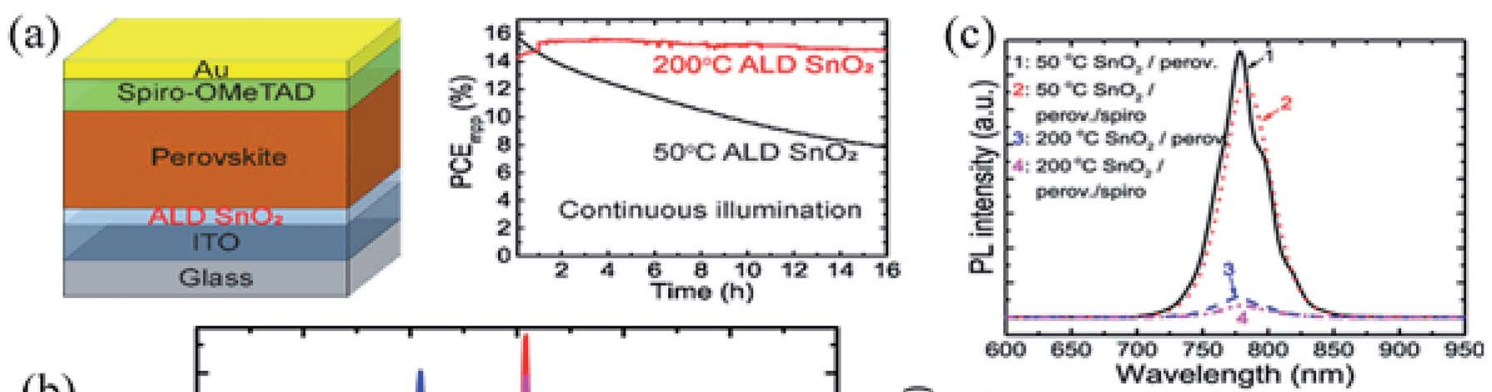

(b)
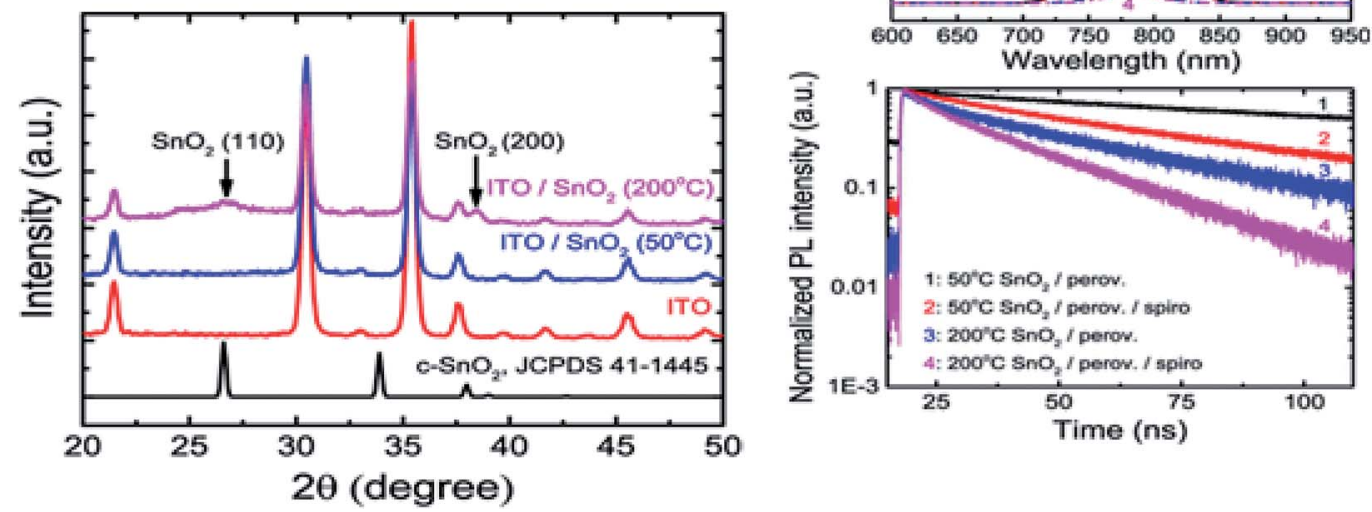

Fig. 24 (a) Schematic representation of plasma-assisted atomic layer deposition of the $\mathrm{SnO}_{2}$ layer (glass/ITO/ALD SnO $2 / \mathrm{CH}_{3} \mathrm{NH}_{3} \mathrm{~Pb}{ }_{3} / \mathrm{spirO}-$ $\mathrm{OMeTAD} / \mathrm{Au}$ ) with its PCE performance; (b) XRD analysis of $\mathrm{SnO}_{2}$ at different annealing temperatures; and (c) steady state and time resolved photoluminescence of $\mathrm{SnO}_{2}$, presented with permission and copyright. ${ }^{301}$ 
$16.2 \%$, a $V_{\mathrm{OC}}$ of $1.086 \mathrm{~V}$, a $J_{\mathrm{SC}}$ of $21.4 \mathrm{~mA} \mathrm{~cm}^{-2}$, and an $\mathrm{FF}$ of $70 \%$ for the $\mathrm{SnO}_{2}$ film annealed at $50{ }^{\circ} \mathrm{C}$ and a PCE of $16.1 \%$, a $V_{\mathrm{OC}}$ of $1.061 \mathrm{~V}, \mathrm{a} J_{\mathrm{SC}}$ of $21.3 \mathrm{~mA} \mathrm{~cm} \mathrm{~cm}^{-2}$, and an $\mathrm{FF}$ of $71 \%$ for the $\mathrm{SnO}_{2}$ film annealed at $200{ }^{\circ} \mathrm{C}$ (Fig. 24). ${ }^{301}$ The improvement of device performance after thermal treatment at $50{ }^{\circ} \mathrm{C}$ is due to the presence of a conduction band offset of $\sim 0.69 \mathrm{eV}$ at the $\mathrm{SnO}_{2} /$ perovskite interface. On the other hand, there is a negligible conduction band offset found after thermal treatment at $200{ }^{\circ} \mathrm{C}$. It is also worth noting that the stability of the device significantly depends on the thermal treatment. PSCs retain their initial performance over $16 \mathrm{~h}$ under continuous one sun illumination in an inert atmosphere for the $\mathrm{SnO}_{2}$ treated at $200{ }^{\circ} \mathrm{C}$, whereas the PCE decreases by $\sim 50 \%$ for the device with $\mathrm{SnO}_{2}$ treated at $50{ }^{\circ} \mathrm{C}$.

\section{7. $\mathrm{SnO}_{2}$ for the sustainable building technology}

\subsection{Transparent/semi-transparent BIPV devices}

An emerging application of transparent conducting $\mathrm{SnO}_{2}$ films is in the field of transparent photovoltaics (TPV). ${ }^{309}$ Unlike the conventional thin-film solar cells which are typically opaque devices with a dark-colored absorber and one reflective cathode, the TPV devices consist of a transparent cathode and a lighttransmitting absorber on a transparent substrate. This new category of solar cells has generated a great deal of research interest in the past several years being partially or fully transmitting to visible sunlight. The TPV devices can be integrated into smart windows or energy harvesting facades during the construction of energy-efficient (green) buildings. ${ }^{\mathbf{3 1 0}}$ These building integrated photovoltaic (BIPV) devices offset the electricity consumed by various building services and reduce the overall carbon footprint of the building. Carbon mitigation is expected to be substantial because a high percentage of electricity generated in a developed economy is used in buildings. BIPV provides an important complement to the decentralized generation of electricity from building applied photovoltaics (BAPV) such as retrofitted roof top solar modules by making use of the large amount of vertical or slanted surfaces on the exterior of multi-storey buildings. TPV equipped windows can limit the amount of heat entering the interior of a building. In addition, for fully transparent TPV devices, the incorporation of these devices will not only adversely alter the aesthetic appeal of the architecture but also can be readily adopted in energyefficient buildings.

Before reviewing the recent developments in TPV devices fabricated based on $\mathrm{SnO}_{2}$ coated substrates, it is important to mention the key device characteristics of TPV devices, namely (1) visible light-transmitting property and (2) power conversion efficiency (PCE), which is a necessary but not sufficient parameter for describing the TPV device performance. Two additional parameters, namely the average visible transmittance (AVT) and the color rendering index (CRI) also need to be determined. The PCE has the same definition as in conventional photovoltaics which is the percentage of the incident solar irradiance $\left(\mathrm{W} \mathrm{cm}^{-2}\right)$ that is converted into electrical power under standard testing conditions (1 sun, AM1.5G, $25^{\circ} \mathrm{C}$ ). Here, the electrical power is the power per unit area at the maximum power point $P_{\mathrm{MPP}}$ given by $P_{\mathrm{MPP}}=J_{\mathrm{SC}^{-}}$ $V_{\mathrm{OC}} \mathrm{FF}$, where $J_{\mathrm{SC}}$ is the short circuit current density, $V_{\mathrm{OC}}$ is the open-circuit voltage and FF is the fill factor of the TPV device. The AVT of a TPV device is usually defined as the average value of the optical transmittance over the spectral range 370$740 \mathrm{~nm}$. It is important to realize that to be aesthetically appealing, the light transmitted through the TPV device must be neutral colored or, in other words, the CIE color coordinates of the transmitted light should be located close to that of natural sunlight. Otherwise, the device will appear tinted and this is undesirable from a building occupant standpoint especially for devices mounted over a window. The color neutrality of a TPV device is described as in solid-state lighting by the CRI. The CRI quantitatively describes how closely the incident light source can render the true color of an object. By definition, the CRI of natural white light is 100, and this serves as the benchmark against which the color rendering of other artificial light sources is evaluated. It is extremely challenging to concurrently optimize all three TPV device parameters (PCE, AVT, and CRI) in the same device. This is because of the inevitable tradeoff between the PCE and the AVT. When the device is optimized for PCE, the thicker absorber layer will necessarily result in a lower AVT and vice versa. Nevertheless, some important breakthroughs have recently been achieved.

As discussed in the review article by Traverse et al., ${ }^{\mathbf{3 1 0}} \mathrm{TPV}$ devices can be classified into "non-wavelength selective" and "wavelength selective" categories. Non-wavelength selective TPV devices make use of absorbers with broadband absorption spectra. Device transmittance is realized by using ultrathin absorbers $(\ll 100 \mathrm{~nm})$ and transparent electrodes. However, since the absorber has absorption in the visible region, and the absorption spectrum usually varies with the wavelength, these devices are often not neutral colored. This is the reason why they are often called "semi-transparent solar cells". Wavelength selective TPV devices, on the other hand, have bespoke absorbers that absorb strongly either at the near infra-red (NIR) or near ultra-violet (NUV) spectral regions. In the AM1.5G spectrum, the NIR region is broader than the NUV; therefore, there is a higher flux of IR photons available for energy harvesting. For each type of selective absorber, the energy bandgap of the absorber should be such that there is no significant absorption of visible light. As a result, the ideal wavelengthselective TPV device appears transparent to the human eye and has a neutral color.

In terms of absorber materials, TPV devices reported thus far are mainly based on thin-film halide perovskite and organic semiconductor absorbers. The inorganic-organic halide perovskite semiconductors are widely studied for TPV devices because there are many solution processing and vacuum deposition methods for preparing these semiconductors. For the solution processing approach, the precursor chemicals are of low-cost and the deposition techniques involved are relatively straightforward. Solution processing does not require high temperature or high vacuum conditions. The synthesized halide perovskite semiconductors possess direct bandgaps that are 
tunable and can be well matched to the solar spectrum by adjusting either the cation or anion chemical composition (compositional engineering). The mixed cation or anion in the material further enhances the tunability of perovskite semiconductors. The perovskite semiconductors also have outstanding absorption coefficients and long minority carrier diffusion lengths $(\sim 1 \mu \mathrm{m})$, which are conducive to high $J_{\mathrm{SC}}$ and $V_{\text {OC }}$. At present, the opaque halide perovskite thin-film solar cells have the highest reported PCE $(>20 \%)$. This rapidly evolving technology is expected to be eventually integrated with conventional silicon solar cells.

Although most TPV devices are based on halide perovskite materials, there have also been studies focusing on organic thin-film heterojunctions. The critical advantage of organic semiconductors is that the energy gap and the energy of the frontier orbitals can be tuned by molecular design, and some organic semiconductors can be solution-processed or printed. However, compared with the chemical precursors of perovskite semiconductors, the cost of development of organic absorbers is likely to be high which can raise the cost of a TPV device. An additional fundamental drawback is a tendency for excited electrons and holes to spontaneously form Frenkel excitons within an organic absorber which requires sufficient energy to dissociate. Usually, dissociation occurs at the 'effective electric field' of a heterojunction formed between a donor (p-type) and an acceptor (n-type) organic layer where the lowest unoccupied molecular orbital (LUMO) of the donor is higher in energy than the LUMO of the acceptor. When a Frenkel exciton diffuses to a heterojunction, the electron within the exciton will spontaneously transfer to the acceptor because of the energy offset. However, this energy loss results in both lower $V_{\text {OC }}$ and device PCE. At present, the PCE of state-of-the-art organic solar cells is substantially lower than that of halide PSCs.

\subsection{Non-wavelength selective TPV devices}

Since methylammonium lead iodide $\left(\mathrm{MAPbI}_{3}\right)$ is the most widely studied halide perovskite, initial efforts were devoted to developing non-wavelength selective (semi-transparent) PSCs based on $\mathrm{MAPbI}_{3}$ absorbers. In ref. 311, Gaspera and co-workers reported a semi-transparent $\mathrm{MAPbI}_{3}$ TPV device with the structure $\quad \mathrm{FTO} / \mathrm{TiO}_{2} / \mathrm{MAPbI}_{3} /$ spiro-OMeTAD $/ \mathrm{MoO}_{3} / \mathrm{Au} / \mathrm{MoO}_{3}$. The dense $\mathrm{TiO}_{2}$ ETL was screen printed onto the FTO substrate, while both the MAPbI ${ }_{3}$ absorber and the hole transport layer spiro-OMeTAD were deposited by solution-based spin coating. The main feature of this device is a thermally evaporated dielectric-metal-dielectric (DMD) structure to enhance the transmittance of the thin precious metal cathode. When properly designed, light transmission can be increased in a DMD structure because of the interference effects associated with the two dielectric layers. The thickness of the layers in the DMD structure needs to be carefully designed for optimizing the transmittance of the DMD. Since the thickness of the DMD layers also affects the photovoltaic properties of a PSC with a DMD cathode, Gaspera et al. varied the bottom $\mathrm{MoO}_{3}$ layer thickness of the DMD stack systematically. When the bottom $\mathrm{MoO}_{3}$ layer was not deposited, $\mathrm{Au}$ island nucleation formed on the spiro-OMeTAD with a higher sheet resistance. PSCs with a $300 \mathrm{~nm}$ absorber thickness fabricated without bottom $\mathrm{MoO}_{3}$, therefore, have higher series resistance and inferior PCE $(<1 \%)$. On the other hand, when a thin bottom $\mathrm{MoO}_{3}$ layer $(1-20 \mathrm{~nm})$ was first deposited on spiro-OMeTAD, both the morphology and sheet resistance of the Au film improved significantly. As a result, the as-constructed PSCs exhibit lower series resistance and higher PCE (11\%). When the bottom $\mathrm{MoO}_{3}$ layer thickness exceeds $20 \mathrm{~nm}$, there is an increase in series resistance and a decrease in the PCE.

It highlighted that the deposition of quality perovskite films is not trivial. However, by using gas-assisted solution processing, films comparable in quality to $\mathrm{MAPbI}_{3}$ absorbers deposited by vacuum evaporation techniques can be obtained. Since $\mathrm{MAPbI}_{3}$ absorbs visible light strongly, the thickness of $\mathrm{MAPbI}_{3}$ had to be reduced from 289 to $54 \mathrm{~nm}$ by lowering the solution concentration during the fabrication of semi-transparent $\mathrm{MAPbI}_{3}$ solar cells. This can be seen from the cross-section scanning electron micrograph presented in Fig. 25a. As the $\mathrm{MAPbI}_{3}$ thickness decreases, there is a reduction in grain size (Fig. 25b). The top electrode of these devices consists of an optimized DMD stack: $5 \mathrm{~nm}$ bottom $\mathrm{MoO}_{3}, 10 \mathrm{~nm} \mathrm{Au}$, and $35 \mathrm{~nm}$ top $\mathrm{MoO}_{3}$. When the $\mathrm{MAPbI}_{3}$ thickness is reduced from 289 to $54 \mathrm{~nm}$, the average batch PCE decreased from $11.7 \%$ to $4.6 \%$, while the AVT increased from $7 \%$ to $31 \%$. The decrease in average PCE for the reported devices is mainly due to a decrease in $J_{\mathrm{SC}}$ (Fig. 25c). Since there is significant variation in the incident photon to electron conversion efficiency (IPCE) and the transmittance in the visible region (Fig. 25d and e), there is a visible tint in the $55 \mathrm{~nm}$ semi-transparent device (see the inset of Fig. 25c). The tradeoff between PCE and AVT, as mentioned earlier, can be seen clearly in Fig. 25f, where parameters from ref. 311 are compared with data from earlier publications. Gasassisted solution processing technique has been used to deposit high-quality, continuous $\mathrm{MAPbI}_{3}$ absorber films. ${ }^{312}$

Eperon et al. investigated the use of formamidinium lead iodide $\left(\mathrm{FAPbI}_{3}\right)$ as the absorber in an effort to develop neutralcolored semi-transparent PSCs. ${ }^{313}$ Due to the fact that the formamidinium cation is larger than the methylammonium ion, the energy band gap of $\mathrm{FAPbI}_{3}$ is reduced to $1.48 \mathrm{eV}$ relative to $1.57 \mathrm{eV}$ for $\mathrm{MAPbI}_{3}$. Other advantages of $\mathrm{FAPbI}_{3}$ in PV applications include greater thermal stability and photostability under illumination. A modified excess organic precursor process using both MAI and FAI in the low vapor pressure solvent dimethyl sulfoxide (DMSO) was used to synthesize $\mathrm{FAPbI}_{3}$ films with an island morphology that is favorable for solar energy conversion. The MAI precursor plays a sacrificial role in the synthesis and is supposed to allow a by-product of the reaction to be removed more easily. The two organic precursors MAI and FAI and $\mathrm{PbCl}_{2}$ were spin-coated followed by thermal annealing. To compare the photovoltaic performance of $\mathrm{FAPbI}_{3}$ and $\mathrm{MAPbI}_{3}$ absorbers deposited by this process, two series of semitransparent planar heterojunction devices with the structure $\mathrm{FTO} / \mathrm{TiO}_{2} / \mathrm{FAPbI}_{3}$ (or) $\mathrm{MAPbI}_{3} /$ spiro-OmeTAD/Au were fabricated. The use of an Au cathode, however, resulted in a nonneutral color. ${ }^{314}$ Photovoltaic performance parameters extracted from $J-V$ characteristics under 1 sun, AM1.5G irradiance 


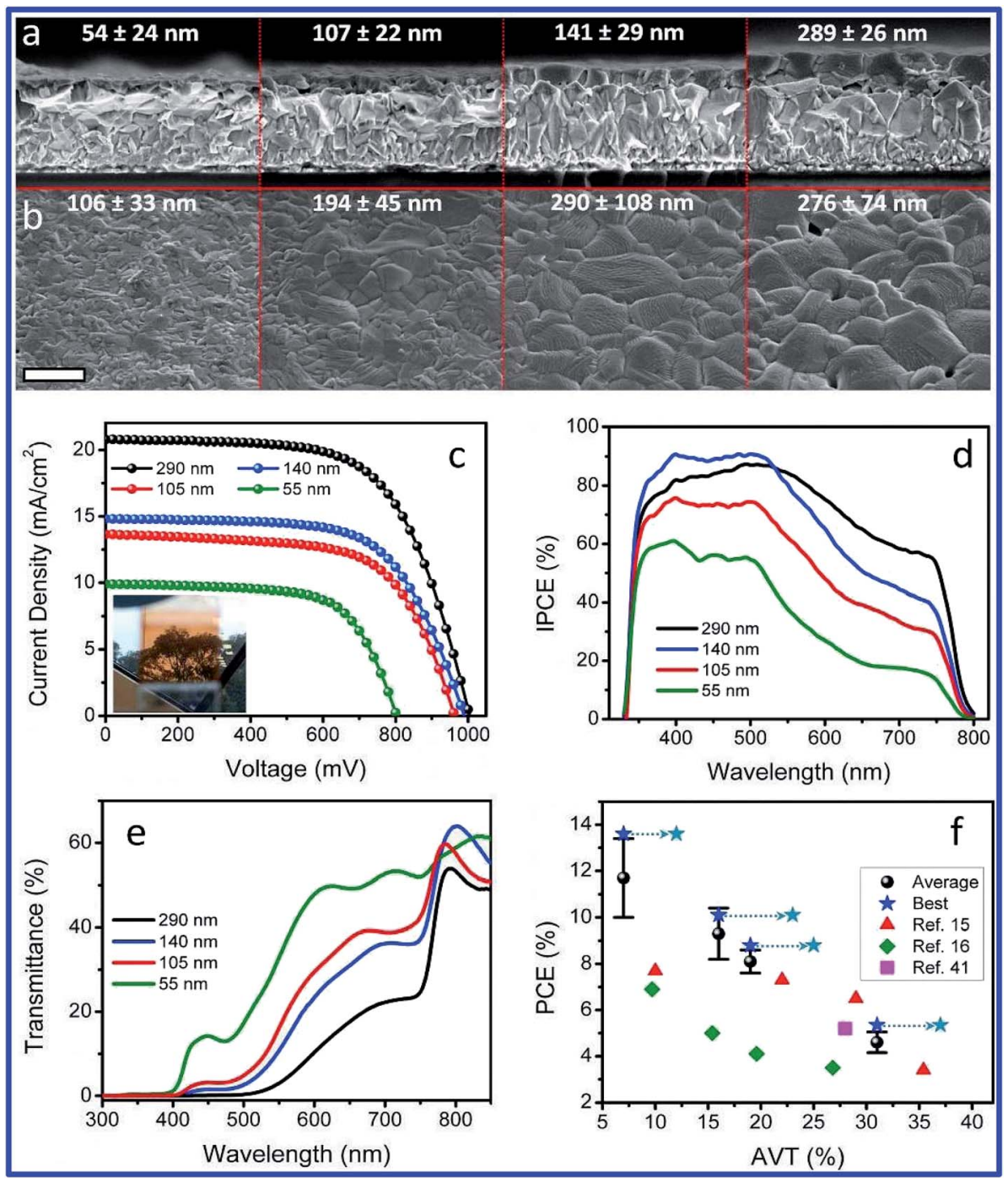

Fig. 25 (a) Cross-section SEM (b) top view SEM, (c) current density voltage characteristics, (d) IPCE spectra, (e) transmittance spectra and (f) PCE versus AVT plot for $\mathrm{MAPbl}_{3}$ thin-film solar cells with different absorber layer thicknesses and a DMD cathode, ${ }^{311}$ presented with permission and copyright.

show that the $J_{\mathrm{SC}}, V_{\mathrm{OC}}, \mathrm{FF}$, and $\mathrm{PCE}$ of the $\mathrm{FAPbI}_{3}$ device are all higher than those of the $\mathrm{MAPbI}_{3}$ device. The external quantum efficiency (EQE) spectra show that the higher $J_{\mathrm{SC}}$ of the $\mathrm{FAPbI}_{3}$ device is due to a longer cutoff wavelength because of its narrower bandgap. Another important aspect of $\mathrm{FAPbI}_{3}$ is that the $J-V$ characteristics are less prone to hysteresis, and the stabilized PCE is closer to the PCE deduced from a rapid $J-V$ scan. These results show that $\mathrm{FAPbI}_{3}$ is a better absorber for highperformance semi-transparent PSCs.

Low-cost color-neutral semi-transparent $\mathrm{FAPbI}_{3}$ solar cells were realized by Eperon et al. using a transparent conductive laminate cathode consisting of a nickel mesh within a PET film. ${ }^{313}$ This transparent laminated cathode (TLC) was attached to the device using a pressure-activated adhesive comprising PEDOT:PSS and acrylic glue. The optical properties of the $\mathrm{FAPbI}_{3}$ device with the TLC attached were characterized by optical transmission and measurement of CIE color coordinates. The attachment of the TLC reduces the average transmittance (AVT) of the FTO and the device layers from $34.2 \%$ to $28.1 \%$. The color coordinates of light transmitted through the TLC, active layer and FTO substrate are close to that of the incident AM1.5G simulated sunlight. This shows that the semi-transparent $\mathrm{FAPbI}_{3}$ device with TLC is neutral colored. The measured PCE of this neutral colored device based on $\mathrm{FAPbI}_{3}$ is $5.2 \%$, while the AVT is only $28 \%$.

In addition to halide perovskites, non-wavelength selective TPV devices have also been reported using organic semiconductors as an absorber. In ref. 315, Upama et al. fabricated semi-transparent organic solar cells using a bulk heterojunction (BHJ) photosensitive layer comprising a blend of PTB7 (poly\{4,8bis[(2-ethylhexyl)oxy]benzo[1,2- $\left.b: 4,5-b^{\prime}\right]$ dithiophene-2,6-diyl-alt3-fluoro-2-[(2-ethylhexyl)carbonyl]thieno[3,4-b]thiophene-4,6diyl $)$ ) and $\mathrm{PC}_{71} \mathrm{BM}\left((6,6)\right.$-phenyl $\mathrm{C}_{71}$ butyric acid methyl ester). The photosensitive ink containing PTB7 (donor) and $\mathrm{PC}_{71} \mathrm{BM}$ (acceptor) were first deposited onto $\mathrm{ZnO}$ coated ITO substrates through spin coating. This is followed by the deposition of 
a $\mathrm{MoO}_{3}(6 \mathrm{~nm}) / \mathrm{Ag}(7 \mathrm{~nm}) / \mathrm{MoO}_{3}(40 \mathrm{~nm})$ OMO cathode to enhance transparency. Starting with the optimized thickness of $200 \mathrm{~nm}$ for the $\mathrm{BHJ}$ layer of opaque devices, the photoactive layer was reduced to $170 \mathrm{~nm}$ and $130 \mathrm{~nm}$ by varying the spin speed, while the thickness of the $\mathrm{MoO}_{3} / \mathrm{Ag} / \mathrm{MoO}_{3}$ layers was kept constant. The PV parameters and the AVT of all three devices were measured and compared with simulation. As the BHJ layer thickness decreased from $200 \mathrm{~nm}$ to $130 \mathrm{~nm}$, the AVT increased from $18.3 \%$ to $24.9 \%$. This increase occurred mainly in the shorter wavelength region (370-600 $\mathrm{nm}$ ). The PCE of the champion device for each $\mathrm{BHJ}$ thickness decreased from $5.00 \%$ $(200 \mathrm{~nm})$ to $3.82 \%(130 \mathrm{~nm})$. The decrease in PCE is mainly due to a decrease in $J_{\mathrm{SC}}$ resulting from reduced photo-generation of excitons.
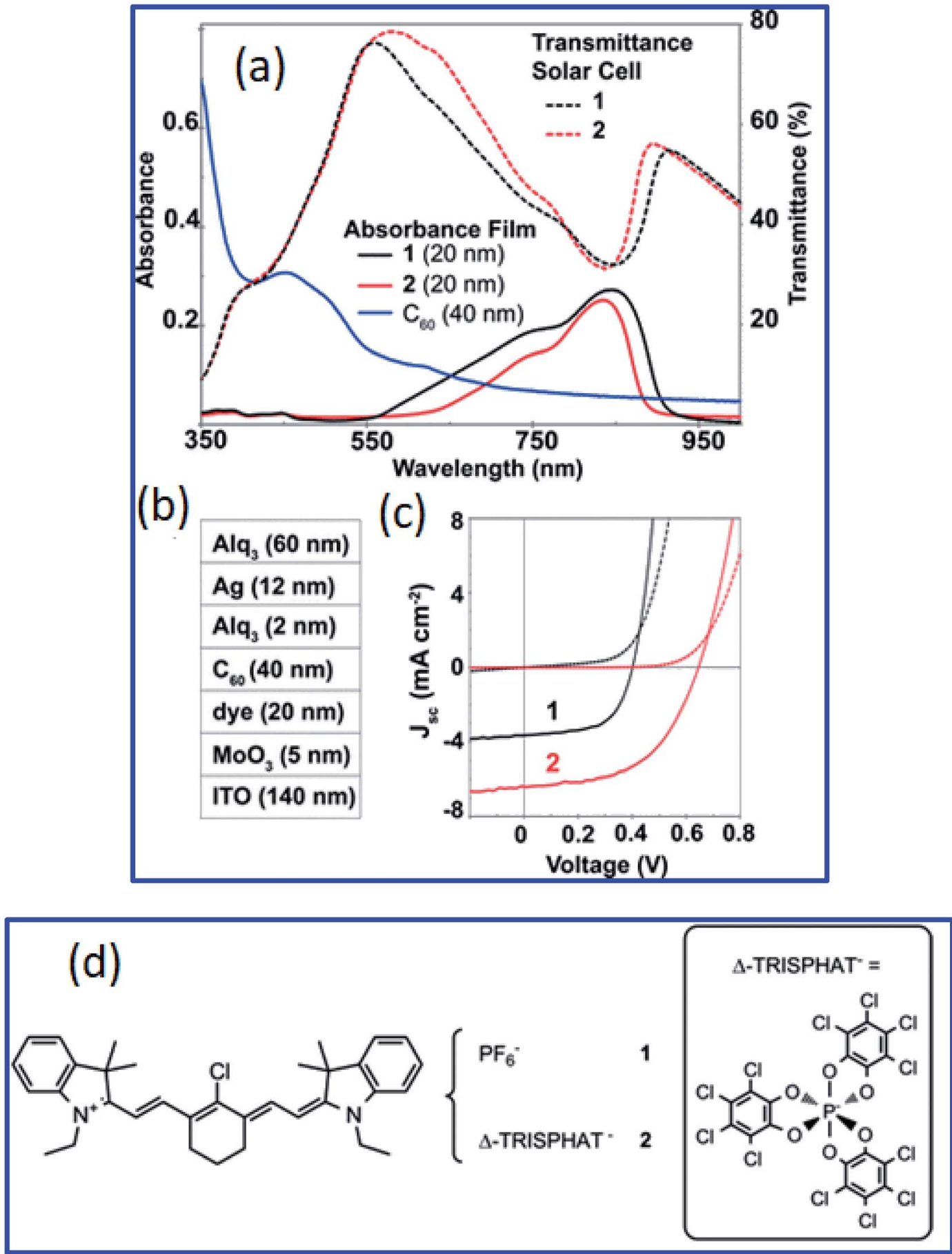

Fig. 26 (a) Solar cell transmittance spectra, film absorbance spectra for compound 1 and compound 2, (b) device structure of the semitransparent NIR absorbing heptamethine dye solar cell, and (c) light and dark J-V characteristics. (d) Molecular structure of the cationic heptamethine dye and the $\Delta$-TRISPHAT ${ }^{-}$counterion used for the fabrication of non-wavelength selective $\mathrm{TPV}^{\text {devices. }} \mathrm{Both}^{\mathrm{PF}}{ }_{6}{ }^{-}$and $\Delta-\mathrm{TRISPHAT}^{-}$ are introduced by the ion exchange reaction, ${ }^{316}$ presented with permission and copyright. 
Unlike other literature, the CRI of these devices was also reported by Upama et al. $;^{315}$ when the $\mathrm{BHJ}$ layer thickness was increased from 130 to $200 \mathrm{~nm}$, the CRI decreased from $66 \%$ to $57 \%$. This shows that there is a separate tradeoff between the PCE and the CRI of these semi-transparent devices. The relatively low CRI is due to strong visible light absorption around 600-700 nm.

\subsection{Wavelength selective TPV devices}

An early example of a wavelength selective TPV device is the NIR absorbing solar cell with heptamethine dye as an absorber, as reported by Véron et al. ${ }^{316}$ The heptamethine dye is a member of the polymethine dyes and is cationic. This means that after synthesis, the heptamethine cation must have a corresponding anion to maintain charge neutrality. The chemical nature of the counterion is known to affect the photophysical properties of the heptamethine dye. ${ }^{316}$

Although the optical absorption spectra of compounds 1 and 2 dissolved in various organic solvents are quite similar, the absorption spectra of $20 \mathrm{~nm}$ thin films of compounds 1 and 2 deposited on glass substrates were found to be affected by the counterion (Fig. 26a). Compound 1 shows stronger absorption than compound 2 from $550 \mathrm{~nm}$ to $900 \mathrm{~nm}$ and has an absorption peak at $850 \mathrm{~nm}$ that is slightly red-shifted from the absorption peak of compound 2. For both compounds, absorption occurs mainly in the NIR region. Bilayer heterojunction devices with the structure ITO $/ \mathrm{MoO}_{3} / \mathrm{dye} / \mathrm{C}_{60} / \mathrm{Alq} \mathrm{q}_{3} / \mathrm{Ag} /$ $\mathrm{Alq}_{3}$ were fabricated using both compound 1 and compound 2 for the dye layer (Fig. 26b). For compound 1, the best performing semi-transparent cell has a PCE of $0.9 \%$ and an AVT (450-670 nm) of $62 \%$. The corresponding values for the best semi-transparent cell fabricated from compound 2 are $2.2 \%$ and $66 \%$, respectively. The maximum transmittance is $75 \%$ at $\sim 590 \mathrm{~nm}$. Since the only difference between semi-transparent cells made from compounds $\mathbf{1}$ and $\mathbf{2}$ is the counterion, the counterion influences the PV properties. As shown by the $J-V$ characteristics (Fig. 26c), compound 2 has both higher $J_{\mathrm{SC}}$ and $V_{\text {OC }}$. This is attributed to the reduced bimolecular recombination and reduced current shunts. Note that both devices in this work are semi-transparent because of the significant absorption tail in the visible region for both compounds, which thus renders these cells non-neutral colored. Véron et $a l^{316}$ performed an ion exchange reaction for the $\mathrm{I}^{-}$ion after the Knoevenagel reaction was performed to synthesize the heptamethine dye core. The original $\mathrm{I}^{-}$anion was exchanged by (i) $\mathrm{PF}_{6}{ }^{-}$and (ii) the organic anion $\Delta$-TRISPHAT ${ }^{-}$(Fig. 26d). These two organic ionic salts are hereafter referred to as compound $\mathbf{1}$ and compound 2, respectively.

In 2017, Davy et al. reported another organic TPV device to selectively harvest NUV photons in sunlight to generate electrical power for driving an electrochromic window (ECW) device. ${ }^{317}$ When a bias voltage is applied, the ECW device will darken within seconds because of the electrochromic property of the active layer in the ECW. The combination of wavelengthselective TPV and ECW devices that operate independently at different spectral regions allows smart windows that can actively regulate the amount of visible and NIR light entering a building without using power from the grid to be realized. This can in turn reduce the amount of power used for cooling the building. Both the donor and the acceptor of the small molecule organic heterojunction TPV device fabricated by Davy et al. are functionalized derivatives of a polyaromatic hydrocarbon molecule called contorted hexabenzocoronene (cHBC). The special properties of cHBC include a wide electronic bandgap (3 eV), an extremely high absorption coefficient $\left(200000 \mathrm{~cm}^{-1}\right)$ and an absorption maximum at $386 \mathrm{~nm} \cdot{ }^{318}$ The cHBC molecule can be easily functionalized and one donor derivative (D, p-type) and two acceptor derivatives (A1 and A2, ntype) were synthesized. The donor contains oxygen heteroatoms, while both acceptors contain chlorine. Both D and A1 and $\mathrm{A} 2$ are thermally stable and can be deposited as thin films by thermal evaporation in a vacuum. The energy levels of the HOMO and the LUMO of the donor and acceptors which are crucial to the design of organic PV devices were measured using UPS and inverse photoemission. The LUMO offset values for D/ $\mathrm{A} 1$ and $\mathrm{D} / \mathrm{A} 2$ are $0.5 \mathrm{eV}$ and $0.8 \mathrm{eV}$, respectively, and are much smaller than the same offset for $\mathrm{D} / \mathrm{C}_{60}$, which is $1.4 \mathrm{eV}$. This small offset results in a smaller energy loss upon exciton dissociation and a cell with higher power output. The absorption coefficient spectra of $\mathrm{D}, \mathrm{A} 1$, and $\mathrm{A} 2$ consist of sharp peaks in the NUV spectral region (372-409 $\mathrm{nm}$ ) and decrease rapidly beyond $450 \mathrm{~nm}$, suggesting that the donor and acceptors are acting in a complementary manner as NUV selective absorbers.

Planar heterojunction solar cells with the structure $\mathrm{Si} /$ $\mathrm{MoO}_{3} / \mathrm{D} / \mathrm{A} 1(\mathrm{~A} 2) / \mathrm{BCP} / \mathrm{Al}$ were fabricated to evaluate the $\mathrm{PV}$ performance of the D/A1 and D/A2 heterojunctions. The $V_{\mathrm{OC}}$ of the devices containing the D/A1 and D/A2 junctions are 1.63 and $1.46 \mathrm{~V}$, respectively, which are much higher than the $V_{\mathrm{OC}}$ of the reference $\mathrm{D} / \mathrm{C}_{60}$ junction $(0.82 \mathrm{~V})$. It is worth pointing out that the $V_{\text {OC }}$ of D/A1 obtained in this work is one of the highest reported for any thin-film solar cell. The high $V_{\text {OC }}$ exhibited by junctions based on derivatives of cHBC is the direct result of the small energy offset mentioned above and the wide bandgaps of D and A1(A2). The wide bandgap of D and $\mathrm{A} 1(\mathrm{~A} 2)$, however, also increases the transparency of these layers and reduces the $J_{\mathrm{SC}}$. Despite this, the maximum power obtainable from the D/A1 and D/A2 devices is comparable to that of the $\mathrm{D} / \mathrm{C}_{60}$ reference device. The PCE of the D/A1 and D/ $\mathrm{A} 2$ devices under 1 sun, $\mathrm{AM} 1.5 \mathrm{G}$ is in the range of $1.3-1.5 \%$, respectively, and the spectral power responsivity for D/A1 and $\mathrm{D} / \mathrm{A} 2$ is $12.4 \%$ and $14 \%$, respectively.

An area scalable TPV device based on D/A1 was demonstrated by replacing the $\mathrm{Si}$ substrate of the planar heterojunction on ITO glass. To reduce the parasitic series resistance, the ITO was coated with a $30 \mathrm{~nm}$ thick Ag grid with $97 \%$ transparency in the range of 300-800 $\mathrm{nm}$. When the device active area was increased from $1 \mathrm{~cm}^{2}$ to $10 \mathrm{~cm}^{2}$, there is little change in the $J-V$ characteristics under illumination. This shows that the TPV device is scalable to match the ECW device. The area scalability is attributed to the amorphous pinhole-free structure of the cHBC derivative films. Although the AVT of this TPV device was not reported, the transmittance of stacked TPV 
and ECW devices in the bleached state ranges from $20 \%$ to $70 \%$ for the spectral range $400-1000 \mathrm{~nm} .^{317}$

Another NUV harvesting air-stable TPV device based on wide bandgap halide perovskite semiconductors was reported by Liu et al. in 2018. ${ }^{319}$ Although $\mathrm{MAPbI}_{3}$ and $\mathrm{FAPbI}_{3}$ are both excellent absorber materials for high-efficiency thin-film solar cells, their broad absorption bands in the visible region render them less useful in fabricating TPV devices with a high AVT. The highest AVT reported for a semitransparent $\mathrm{MAPbI}_{3}$ device is $\sim 46 \%$. By substituting the iodide ion by smaller halide ions such as bromide and chloride in $\mathrm{MAPbI}_{3}$, the absorption edge can be shifted towards the UV wavelengths. In ref. 319, two mixed halide perovskites with the general formula $\operatorname{MAPbCl}_{3-x} \mathrm{Br}_{x}(x=$ 0 and 0.6$)$ were fabricated by solution-based spin coating. However, the process is more challenging than the spin coating deposition of $\mathrm{MAPbI}_{3}$ because of rapid crystallization and limited solubility of $\mathrm{MAPbCl}_{3}$ precursors in solvents. The specific details of the deposition process were found to have a crucial impact on the quality of the resulting halide perovskite films. After investigating various alternative processes, Liu et al. reported that smooth, uniform and transparent $\mathrm{MAPbCl}_{3-x} \mathrm{Br}_{x}$ films can only be obtained by using a vacuum-assisted solution deposition together with post-deposition through methylamine gas treatment. ${ }^{319} \mathrm{~A}$ blue shift in the optical absorption edge was observed as the bromide content $x$ is decreased. As a result, the $\mathrm{MAPbCl}_{2.4} \mathrm{Br}_{0.6}$ films deposited by this process appear light yellow, while the $\mathrm{MAPbCl}_{3}$ films are transparent.
TPV devices with the structure ITO/PEDOT/MAPbCl ${ }_{3-x} \mathrm{Br}_{x} /$ $\mathrm{C}_{60} / \mathrm{BCP} / \mathrm{Ag} / \mathrm{Alq}_{3}$ were fabricated on ITO glass substrates. Here, $\mathrm{BCP}$ is bathocuproine and $\mathrm{Alq}_{3}$ is tris-(8-hydroxyquinoline) aluminum. The PEDOT layer was used as the HTL and $\mathrm{C}_{60}$ as the ETL. BCP was used in conjunction with $\mathrm{Alq}_{3}$ to enhance the transmittance of the top $\mathrm{Ag}$ electrode by an $\mathrm{OMO}$ thin film structure. The best $\mathrm{MAPbCl}_{3-x} \mathrm{Br}_{x}$ TPV device displayed a PCE of $0.52 \%$, while the champion $\mathrm{MAPbCl}_{3}$ device had a PCE of $0.32 \%$. This is because of the narrower bandgap of the $\mathrm{MAPbCl}_{3-x} \mathrm{Br}_{x}$ absorber $(2.83 \mathrm{eV})$ relative to that of $\mathrm{MAPbCl}_{3}(3.04 \mathrm{eV})$, which results in a higher $J_{\mathrm{SC}}$. The AVT of the $\mathrm{MAPbCl}_{3-x} \mathrm{Br}_{x}$ device and the $\mathrm{MAPbCl}_{3}$ device calculated from the transmittance spectra are $73 \%$ and $72.1 \%$, respectively (Fig. $27 \mathrm{a}$ and b). These are the highest AVT reported for any TPV device with a transparent active layer. Since both devices are highly transparent in the visible region (Fig. 27c and d), the CRI values are similarly high (94.4 for $\mathrm{MAPbCl}_{3}$ and 93.8 for $\mathrm{MAPbCl}_{3-x} \mathrm{Br}_{x}$ ). These CRI values suggest that light transmitted through these devices can provide the natural color to objects very well (Fig. 27c and d). The main improvement needed for these devices with wide bandgap halide perovskite absorbers includes increasing the internal quantum efficiency which should lead to a higher PCE in the future.

\section{8. $\mathrm{SnO}_{2}$ for energy storage}

Energy from sustainable resources has been considered one of the key challenges for the modern society. ${ }^{320}$ Indeed, a rapid

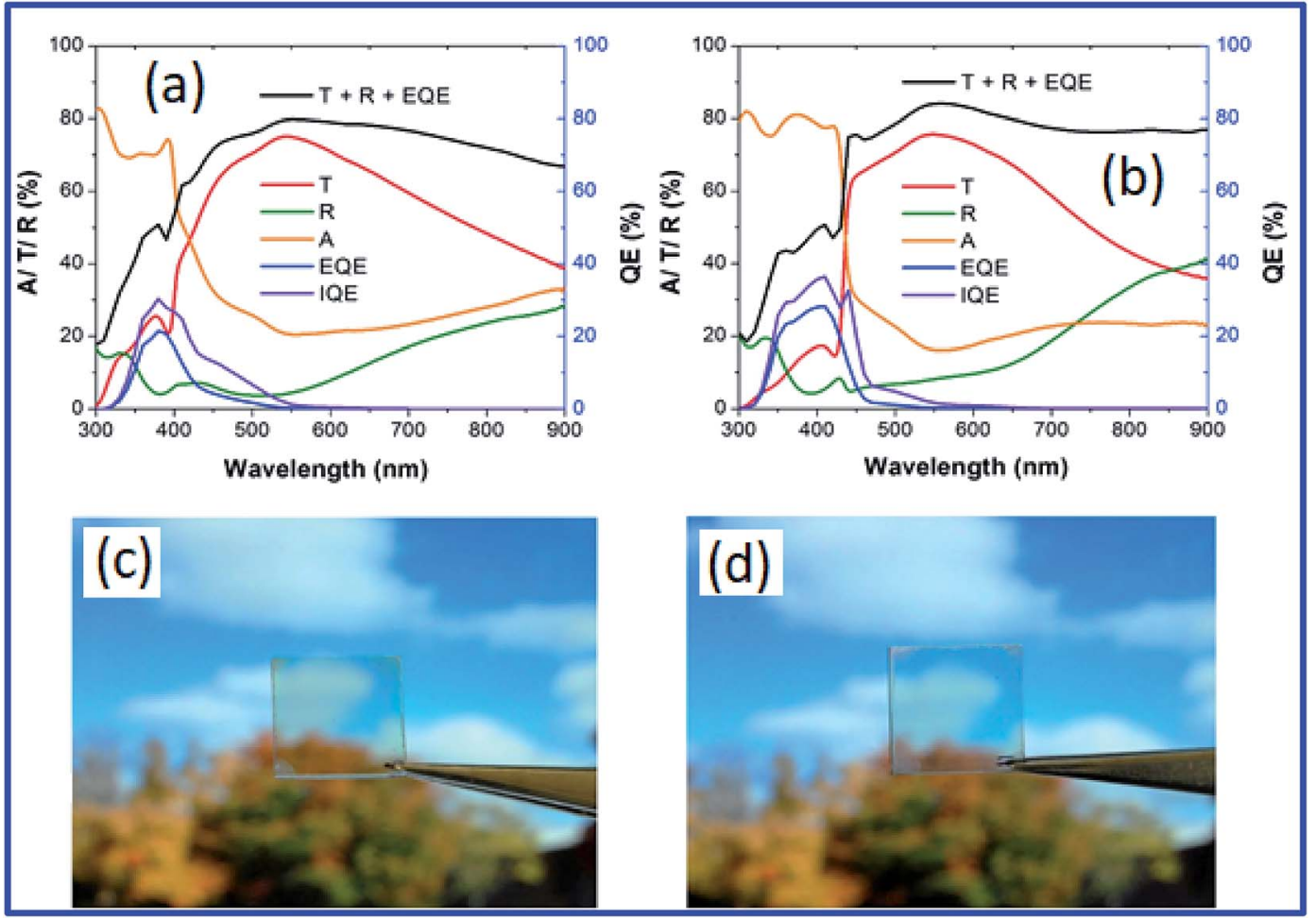

Fig. 27 (a) Absorbance, transmittance, reflectance, external quantum efficiency (EQE) and internal quantum efficiency (IQE) spectra of the $\mathrm{MAPbCl}_{3}$ device, (b) absorbance, transmittance, reflectance, EQE and IQE spectra of the MAPbCl${ }_{2.4} \mathrm{Br}_{0.6}$ device, (c) photograph of the TPV device fabricated from the $\mathrm{MAPbCl}_{3}$ absorber, (d) photograph of the TPV device fabricated from the $\mathrm{MAPbCl}_{2.4} \mathrm{Br}_{0.6}$ absorber, ${ }^{319}$ presented with permission and copyright. 
increase in the global population and growing economic expansion significantly increased the energy consumption which heavily relies on sustainable sources of energy materials and devices. Access to green, affordable, and dispatchable energy that can act as a substitute for fossil fuels is urgently needed. The dramatically rising worldwide concerns on the shortage of clean/renewable energies warrant the novel design and development of advanced nanostructured materials towards fabricating stable energy storage devices. In this regard, electrochemical storage plays an increasingly important role in the design and development of sustainable energy technologies. Among the electrochemical storage, batteries and supercapacitors have been heavily investigated by both industry partners and the research community to meet the on-going demands of electronics (consumer/smart/portable), electric vehicles, etc.321-323 The salient differences between batteries and supercapacitors are that they have different storage mechanisms, device configurations, and fabrication procedures. Typically, batteries can provide high energy density, while supercapacitors offer high power density for charge storage.

\section{1 $\mathrm{SnO}_{2}$ in lithium-ion batteries (LIBs)}

Li-ion batteries (LIBs) are regarded as an advanced promising technology among various electrochemical energy storage applications because of their excellent volumetric and gravimetric power/energy density, extremely low self-discharging characteristics, high stability and long life cycle. ${ }^{324}$ LIBs serve as the dominant power source for a wide range of portable electronics such as mobile phones and laptops. ${ }^{325}$ However, the practical capacity of conventional LIBs is still far below the current market demand in the field of electric vehicles due to their insufficient energy density. Therefore, the design of new electrode materials for the LIBs using emerging advanced nanostructures with improved characteristics is a prerequisite for their realization in real-time energy storage applications that meet the on-going demand of 2-5 times higher energy density. ${ }^{326}$ Over the past decade, significant efforts have been devoted to investigating transition metal oxide $\left(\mathrm{TiO}_{2}, \mathrm{SnO}_{2}\right.$, $\mathrm{MnO}_{2}$, and $\mathrm{Fe}_{2} \mathrm{O}_{3}$ ) based anode materials in LIBs owing to their versatile tunable physical/chemical properties with promising electrochemical performances. Some of the representative specific capacities of nanostructured materials (anode based) include graphene $\left(960 \mathrm{~mA} \mathrm{~h} \mathrm{~g}{ }^{-1}\right),{ }^{327}$ CNTs $\left(1100 \mathrm{~mA} \mathrm{~h} \mathrm{~g}^{-1}\right){ }^{328}$ porous carbon (1100 $\left.\mathrm{mA} \mathrm{h} \mathrm{g}^{-1}\right)$, silicon (4200 $\left.\mathrm{mA} \mathrm{h} \mathrm{g}^{-1}\right),^{329}$ and transition metal oxides (500-1000 $\left.\mathrm{mA} \mathrm{h} \mathrm{g}^{-1}\right)^{330}$ among others. However, inherent limitations of the anode materials such as high volume variation during charging/discharging, capacity fading due to solid-electrolyte interphase (SEI) films, poor electrical conductivity, and low coulombic efficiency must be overcome before these newer anode materials can effectively function in practical LIBs.

Metal-oxide based and sulfide based electrode materials such as molybdenum disulfide $\left(\mathrm{MoS}_{2}\right),{ }^{\mathbf{3 3 1}, \mathbf{3 3 2}}$ manganese dioxide $\left(\mathrm{MnO}_{2}\right),{ }^{333}$ ferric oxide $\left(\mathrm{Fe}_{3} \mathrm{O}_{4}\right),{ }^{334,335}$ and cobalt tetraoxide $\left(\mathrm{Co}_{3} \mathrm{O}_{4}\right)^{336}$ based nanostructured composite materials have been widely studied as storage materials in LIBs because of their abundance, the high theoretical capacity of $992 \mathrm{~mA} \mathrm{~h} \mathrm{~g}^{-1}$ and the low-working potential $(0.6 \mathrm{~V}) \cdot{ }^{337-343} \mathrm{In}$ recent years, $\mathrm{SnO}_{2}$ has garnered considerable attention due to its abundance, low-cost, biological compatibility, and high theoretical/specific capacity (1494 $\mathrm{mA} \mathrm{h} \mathrm{g}^{-1}$ ) (as against graphite $\left(342 \mathrm{~mA} \mathrm{~h} \mathrm{~g}^{-1}\right)$ ). ${ }^{343} \mathrm{SnO}_{2}$ based materials have gained momentum as an alternative anode material in LIBs since the pioneering work of Idota et al. more than two decades ago (1997) who reported a reversible capacity of $600 \mathrm{~mA} \mathrm{~h} \mathrm{~g}{ }^{-1}$. ${ }^{344}$ Typically, $\mathrm{SnO}_{2}$ undergoes 2 major steps in the reaction with lithium ions $\left(\mathrm{Li}^{+}\right)$that includes the conversion reaction and the alloying/de-alloying process. ${ }^{345,346}$

$$
\begin{gathered}
\mathrm{SnO}_{2}+4 \mathrm{Li}^{+}+4 \mathrm{e}^{-} \rightarrow 2 \mathrm{Li}_{2} \mathrm{O}+\mathrm{Sn} \\
\mathrm{Sn}+x \mathrm{Li}^{+}+x \mathrm{e}^{-} \rightarrow \mathrm{Li}_{x} \mathrm{Sn}(0 \leq x \leq 4.4) \\
\mathrm{Li}_{x} \mathrm{Sn} \rightarrow \mathrm{Sn}+x \mathrm{Li}^{+}+x \mathrm{e}^{-}(0 \leq x \leq 4.4)
\end{gathered}
$$

To further improve the surface area and specific capacity of $\mathrm{SnO}_{2}$ based active materials, several strategies have been devised in tuning the morphology and nanostructuring at the nanoscale through hybridization of $\mathrm{SnO}_{2}$ with various suitable supports such as metals, carbon nanostructures, metal sulfides/ phosphides/chalcogenides, conducting polymers, etc.347-349 For instance, compositing with carbon nanostructures (CNTs, graphene, fullerenes) not only helps to tackle the volume expansion during charging/discharging but also serves to improve the overall conductivity. ${ }^{350}$ Various design strategies/ principles of nanostructured $\mathrm{SnO}_{2}$ (nanoparticles/ nanocrystals, nanoflowers, nanotubes, nanoplates, nanospheres, nanowires, quantum dots, etc.) have been employed to fabricate robust $\mathrm{SnO}_{2}$ based anodes, which are considered as one of the promising routes for achieving high coulombic efficiencies and high-specific capacities in LIBs. ${ }^{351-355}$

The majority of the work focuses on modifying $\mathrm{SnO}_{2}$ with various carbon nanomaterials (graphene, mesoporous carbon, CNTs, etc.) with the aim of constructing robust and stable LIBs. ${ }^{338,356-359}$ For example, a 2D porous carbon-coated sandwich structure featuring mesoporous $\mathrm{SnO}_{2} /$ graphene/ $\mathrm{SnO}_{2}$ nanosheets ( $\mathrm{C} @ \mathrm{SnO}_{2}-\mathrm{rGO}-\mathrm{SnO}_{2}$ ) has been rationally designed and fabricated through combined hydrothermal and templateassisted nanocasting impregnation strategies. Wang et al. demonstrated enhanced Li storage capacity in LIBs by judiciously considering the inherent beneficial properties of individual components (Fig. 28). ${ }^{356}$ The authors took advantage of the nanocrystal size and mesoporous structure to design this type of unique hybridized system through utilizing $\mathrm{SiO}_{2}-\mathrm{rGO}-\mathrm{SiO}_{2}$ as a template. The electrochemical performances of various hybrids were compared $\left(\mathrm{GO} / \mathrm{SnO}_{2}, \mathrm{GO}-\mathrm{SnO}_{2} @ \mathrm{C}\right.$, mesoporous $\mathrm{SnO}_{2}-$ rGO-SnO ${ }_{2}$ (c), and porous $\mathrm{C} @ \mathrm{SnO}_{2}-\mathrm{rGO}-\mathrm{SnO}_{2}$ ) and their reaction mechanisms were presented for the lithiation/delithiation processes. Among the investigated electrode materials, $\mathrm{C} @ \mathrm{SnO}_{2}-\mathrm{rGO}-\mathrm{SnO}_{2}$ exhibited excellent reversibility, high rate performance ( $315 \mathrm{~mA} \mathrm{~h} \mathrm{~g}^{-1}$ at $10 \mathrm{~A} \mathrm{~g}^{-1}$ ), and extended cyclability $\left(525 \mathrm{~mA} \mathrm{~h} \mathrm{~g}^{-1}\right.$ after 1200 cycles at $\left.2 \mathrm{~A} \mathrm{~g}^{-1}\right)$.

In another interesting report, monodispersed ultra-small $(\sim 5 \mathrm{~nm})$ nanocrystals of $\mathrm{SnO}_{2}(74.85 \mathrm{wt} \%)$ were uniformly 


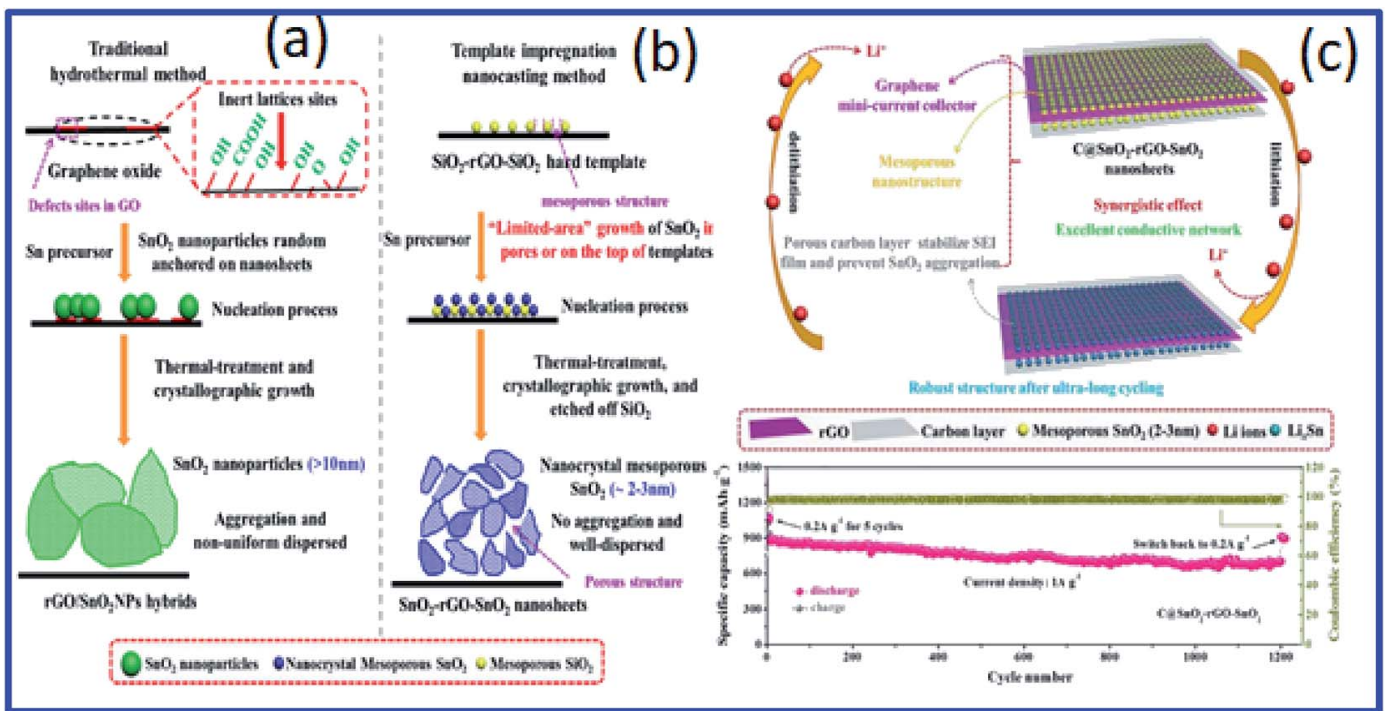

Fig. 28 Schematic representation of the research methodology adopted for the (a) hydrothermal method, (b) nanohard template casting method, and (c) charge/discharge profiles of $\mathrm{CaSnO} 2-\mathrm{rGO}-\mathrm{SnO}_{2}$ at a current density of $1 \mathrm{~A} \mathrm{~g}^{-1}, 356$ presented with permission and copyright.

distributed on co-doped nitrogen/sulfur graphene sheets (NSGS) using the layer by layer (LBL) assembly strategy towards the fabrication of high-performance LIBs with high discharge capacity (2123 $\mathrm{mA} \mathrm{h} \mathrm{g}^{-1}$ at $\left.0.1 \mathrm{~A} \mathrm{~g}^{-1}\right)$, extended cyclability (99.2\% after 500 cycles), and rate capabilities. ${ }^{338}$ Besides, the authors also tested the optimized electrode system (NSGS-8) in Li half/full fuel cells and systematically investigated their electrochemical reaction kinetics. Similarly, hollow $\mathrm{SnO}_{2}$ nanospheres (positively charged) with oxygen vacancies encapsulated by a nitrogen-doped network of graphene (negatively charged) $\left(\mathrm{SnO}_{2-x} / \mathrm{N}-\mathrm{rGO}\right)$ have been prepared by the electrostatic adsorption-induced self-assembly and further tested for their practical utility in LIBs (Fig. 29a-d). Fig. 29b depicts the HR-TEM image of $\mathrm{SnO}_{2-x} / \mathrm{N}$-rGO showing lattice fringes with the interplanar distance $(0.33 \mathrm{~nm})$ corresponding to tetragonal $\mathrm{SnO}_{2}$. The charge/discharge profile and the cycling performance of the investigated materials (bare and carbonbased anodes) and the remarkable rate capability

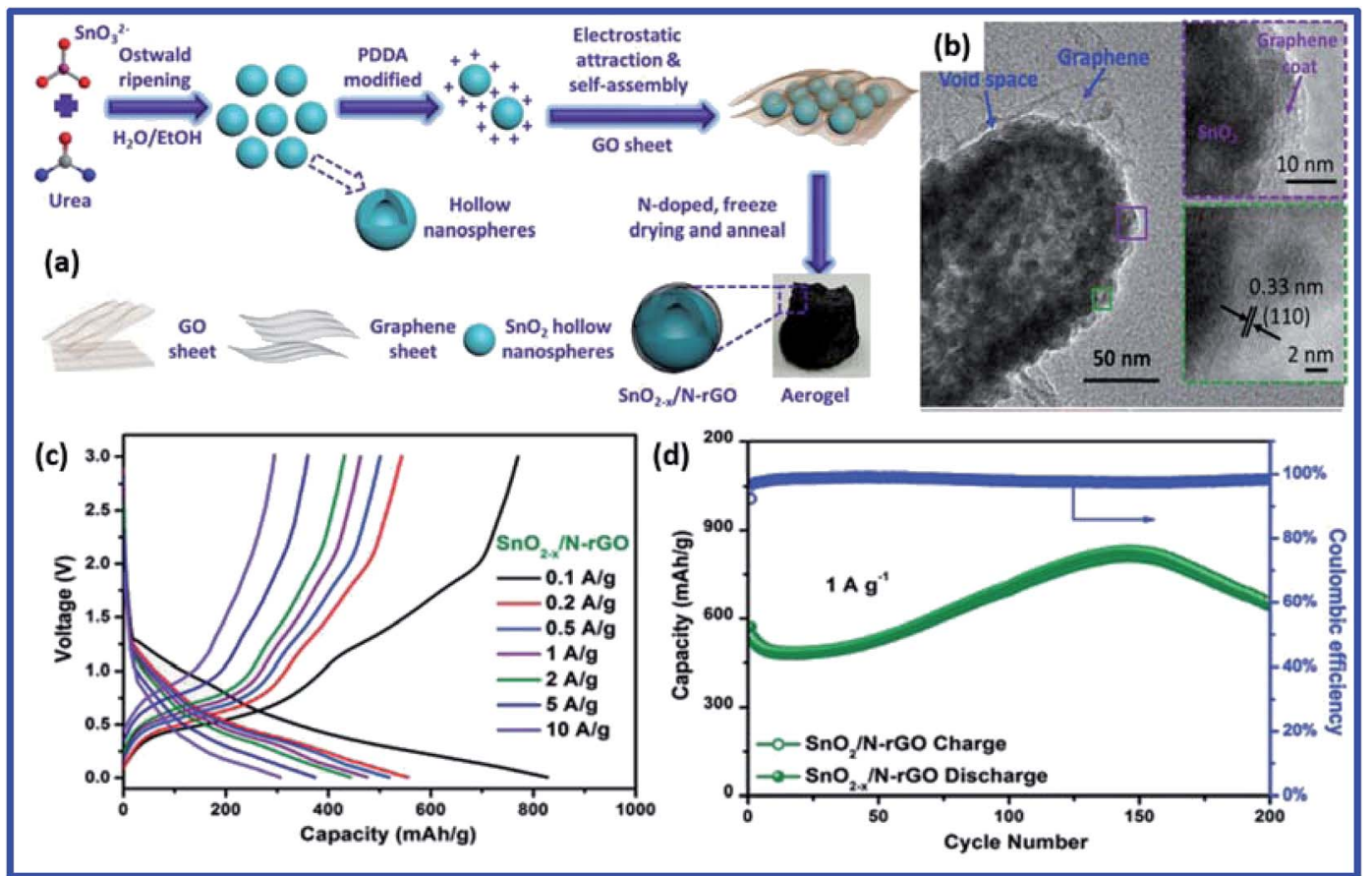

Fig. 29 (a) Scheme showing the preparation of a 3D hierarchical $\mathrm{SnO}_{2-x} / \mathrm{N}-\mathrm{rGO}$ network. (b) TEM images, with the insets showing the HRTEM images, (c) charge-discharge profiles, and (d) cycling performance $\left(0.1 \mathrm{~A} \mathrm{~g}^{-1}\right)$ of $\mathrm{SnO}_{2-x} / \mathrm{N}-\mathrm{rGO}, 357$ presented with permission and copyright. 
(309 $\mathrm{mA} \mathrm{h} \mathrm{g}^{-1}$ at $10 \mathrm{~A} \mathrm{~g}^{-1}$ ) and robust stability (912 $\mathrm{mA} \mathrm{h} \mathrm{g}^{-1}$ even after 500 cycles) (Fig. 29c and d) exhibited by the asconstructed nanostructured $\mathrm{SnO}_{2}$ entrapped by an $\mathrm{N}$-doped graphene composite network envisage the potential prospects of $\mathrm{SnO}_{2}$ in advanced LIBs. ${ }^{357}$

In LIBs, $\mathrm{SnO}_{2}$ is well established as an anode material. However, there are reports where $\mathrm{SnO}_{2}$ nanoparticles were used to modify the conventional cathodes such as lithium cobalt oxide (LCO) ${ }^{360}$ and lithium nickel cobalt manganese oxide, $\mathrm{LiNi}_{1 / 3} \mathrm{Co}_{1 / 3} \mathrm{Mn}_{1 / 3} \mathrm{O}_{2}$ (LNCMO). ${ }^{361}$ Ma et al. demonstrated that a thin layer of $\mathrm{SnO}_{2}$ coating on $\mathrm{LiNi}_{0.5} \mathrm{Mn}_{1.5} \mathrm{O}_{4}$, LNMO, by a facile synchronized coating strategy can enhance its electrochemical performance. ${ }^{362}$ With the help of these coating strategies, they not only were able to coat a thin layer of $\mathrm{SnO}_{2}$ on the surface of the LNMO but also a very small amount of $\mathrm{Sn}^{2+}$ ions can enter the spinel to stabilize and modify the spinel structure. ${ }^{362}$ The battery performance demonstrated that the specific

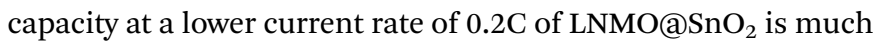
higher than that of the pristine LNMO. For the pristine LNMO, the material is derived from calcination of the precursor at a high temperature. At that temperature, the Ni solubility in the LNMO spinel lattice will decrease and a reduction of $\mathrm{Mn}^{4+}$ to $\mathrm{Mn}^{3+}$ took place, which resulted in the formation of a $\mathrm{Ni}_{x} \mathrm{Li}_{y} \mathrm{O}$ impurity phase. Thus, the obtainable specific capacity of pristine LNMO was lower due to the presence of the $\mathrm{Ni}_{x} \mathrm{Li}_{y} \mathrm{O}$ impurity phase, and a given amount of $\mathrm{Mn}^{3+}$ also existed in the spinel due to charge neutrality, which is different from the ordered $\mathrm{P}_{3} 32$ structure of stoichiometric $\mathrm{LiNi}_{0.5} \mathrm{Mn}_{1.5} \mathrm{O}_{4}$. For LNMO@ $\mathrm{SnO}_{2}$, the $\mathrm{Sn}^{2+}$ doping would inhibit the formation of the inactive $\mathrm{Ni}_{x} \mathrm{Li}_{y} \mathrm{O}$ impurity phase owing to its structural stabilization effect. As a result, the reduction of $\mathrm{Mn}^{4+}$ to $\mathrm{Mn}^{3+}$ in the spinel was inhibited. This decreased content of inactive
$\mathrm{Ni}_{x} \mathrm{Li}_{y} \mathrm{O}$ was responsible for the increased specific capacity of

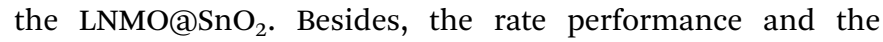

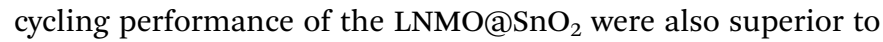
those of the pristine LNMO. The improved cycling performance

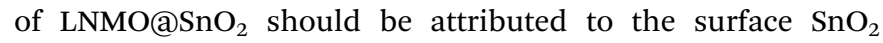
coating layer, which alleviated the interaction between the active material surface and the electrolyte and suppressed the excessive formation of a thick SEI film.

For the superior performance of a LIB, the electrolyte affinity and the thermal stability of the separator are very crucial. In a report, Xiang and co-workers functionalized a polyethylene (PE) separator through binder assisted coating using hollow $\mathrm{SnO}_{2}$ nanoparticles of size around $250 \mathrm{~nm}$ and investigated this functionalized PE separator and LIBs systematically. ${ }^{363}$ It was observed that the $\mathrm{SnO}_{2}$ functionalized $\mathrm{PE}$ separator not only enhanced the electrolyte wettability but also can withstand a temperature of $130{ }^{\circ} \mathrm{C}$, which means that the functionalized PE separator can uptake a higher electrolyte content as a result of which a high rate performance and charge-discharge performance at elevated temperatures were achieved. To evaluate the battery performance of the functionalized separator, it was tested with $\mathrm{LiCoO}_{2}$ half cells at room temperature and $80{ }^{\circ} \mathrm{C}$. At room temperature, both the pristine and functionalized separators showed similar initial discharge capacity as well as similar capacity retention after 100 cycles. However, when the half cells were tested at $80{ }^{\circ} \mathrm{C}$, it was observed that the efficiency of the pristine separator declined rapidly after the 70th cycle, whereas the functionalized separator was stable even after 70 cycles throughout the testing period at $80{ }^{\circ} \mathrm{C}$. This could be attributed to the localized deficiency of the electrolyte in the pristine separator during high temperature cycling of the battery. As the cycling goes on, the electrolyte may redistribute

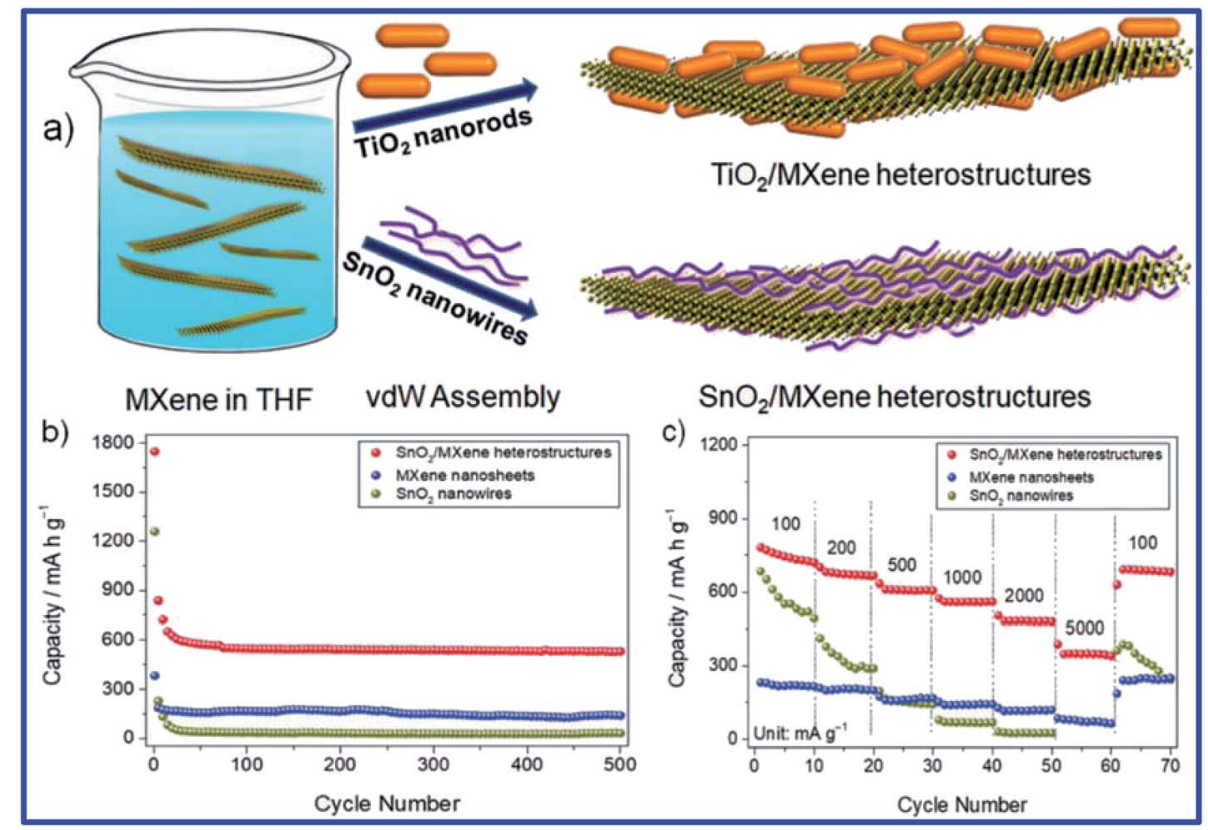

Fig. 30 (a) Scheme showing the self-assembly on MXene nanosheets, (b) their specific capacities at a current density of $1000 \mathrm{~mA} \mathrm{~g}^{-1}$ and (c) rate capabilities for 70 cycles, $^{347}$ presented with permission and copyright. 
in the battery and the capacity of the battery is partly recovered. The excellent half-cell performance of the $\mathrm{SnO}_{2}$ functionalized separator demonstrates the hypothesis that the $\mathrm{SnO}_{2}$ coating layer enhances the electrolyte retention of the PE separator at elevated temperatures. ${ }^{363}$

In yet another interesting report, a general route for the facile, cost-effective preparation of metal oxide $\left(\mathrm{TiO}_{2} / \mathrm{SnO}_{2}\right) /$ MXenes has been reported by Gogotsi et al. through selfassembling $\mathrm{SnO}_{2}$ nanowires on MXene nanosheets through van der Waals interactions towards fabricating high power/ energy, superior rate performance and stable energy storage in LIBs (Fig. 30). ${ }^{347}$ Herein, MXenes $\left(\mathrm{Ti}_{3} \mathrm{C}_{2}\right)$ play the role of a conductive additive to mitigate the agglomeration of $\mathrm{SnO}_{2}$. On the other hand, the use of $\mathrm{SnO}_{2}$ avoids the precipitation and restacking of MXene nanosheets during the Li insertion/ extraction process. A high capacity of $530 \mathrm{~mA} \mathrm{~h} \mathrm{~g}{ }^{-1}$ was observed even after 500 cycles. The comparison of rate capabilities reveals that the as-fabricated $\mathrm{SnO}_{2}$ /MXene heterostructures delivered superior capacities of $720,665,606,560$, 489 , and $310 \mathrm{~mA} \mathrm{~h} \mathrm{~g}^{-1}$ at current densities of 100, 200, 500, 1000,2000 , and $5000 \mathrm{~mA} \mathrm{~g}^{-1}$, respectively, over their nonmodified $\mathrm{SnO}_{2}$ and MXene counterparts. The reduced pathway for lithium diffusion and enhanced active area have been observed to contribute to the remarkable electrochemical performance in the fabricated LIBs.

\section{2 $\mathrm{SnO}_{2}$ in lithium-sulphur batteries (LSBs)}

Lithium-sulphur (Li-S) batteries are also considered as the future of energy storage devices owing to their high theoretical capacitance $\left(\sim 1680 \mathrm{~mA} \mathrm{~h} \mathrm{~g}^{-1}\right.$ for sulfur and $3860 \mathrm{~mA} \mathrm{~h} \mathrm{~g}^{-1}$ for lithium). ${ }^{364}$ Considering an average voltage window of $2.15 \mathrm{~V}$, the theoretical specific energy density of the Li-S batteries was estimated to be $\sim 2500 \mathrm{~W} \mathrm{~h} \mathrm{~kg}{ }^{-1}$, which is beyond that of conventional LIBs $\left(\sim 400 \mathrm{~mA} \mathrm{~h} \mathrm{~g}{ }^{-1}\right)$. Also, sulfur is cheap and earth-abundant, making Li-S batteries a promising low-cost technology for widespread usage. Owing to the strong adsorption capability of lithium polysulphides, $\mathrm{SnO}_{2}$ has been used as a cathode to trap lithium polysulphide which in turn not only reduces the shuttling effect but also enhances the overall performance of the Li-S batteries. ${ }^{365}$ Fig. 31 presents the schematic representation of conventional LIBs, conventional Li-S batteries and $\mathrm{SnO}_{2}$ modified Li-S batteries.
Different from others, $\mathrm{Yu}$ et al. ${ }^{366}$ used an $\mathrm{rGO} / \mathrm{SnO}_{2} / \mathrm{S}$ composite prepared via a one-pot hydrothermal method for use as a cathode and obtained an initial discharge capacity of $1592 \mathrm{~mA} \mathrm{~h} \mathrm{~g}^{-1}$ at $0.1 \mathrm{C}$ which stabilized to $607 \mathrm{~mA} \mathrm{~h} \mathrm{~g}^{-1}$ at $0.2 \mathrm{C}$ over 100 cycles which is more than that of the $\mathrm{rGO} / \mathrm{S}$ cathode (508 $\mathrm{mA} \mathrm{h} \mathrm{g}^{-1}$ at $0.2 \mathrm{C}$ over 100 cycles) and the pristine $\mathrm{S}$ cathode electrode ( $388 \mathrm{~mA} \mathrm{~h} \mathrm{~g}^{-1}$ at $0.2 \mathrm{C}$ over 60 cycles). Fig. 32a presents the schematic representation of $\mathrm{SnO}_{2}$ as a polysulphide immobilizer. From the high resolution transmission electron microscopy (HRTEM) image presented in Fig. 32b, it can be observed that $5 \mathrm{~nm} \mathrm{SnO}_{2}$ nanoparticles are embedded in $\mathrm{rGO}$ sheets along with the loading of synthesized sulfur. The elemental mapping further confirmed the presence of Sn, C, O, and S (Fig. 32c). The formation of lithium polysulphide and lithium sulphide was observed from the cathodic peak position at $2.3 \mathrm{~V}$ and $2.0 \mathrm{~V}$ in the cyclic voltammetry (CV) curve presented in Fig. 32d. During the anodic process, lithium sulphide first converts into lithium polysulphide and then to $\mathrm{S}$, which was confirmed from the presence of anodic peaks at $2.3 \mathrm{~V}$ and $2.4 \mathrm{~V}$, respectively. No redox peaks were observed between $1.7 \mathrm{~V}$ and $2.8 \mathrm{~V}$, which confirmed that $\mathrm{SnO}_{2}$ does not take part in the redox reaction and only acted as a polysulphide immobilizer. From Fig. 32e and f, it can be derived that $\mathrm{SnO}_{2}$ has a positive influence on the electrochemical performance of the $\mathrm{rGO} / \mathrm{S}$ cathode. The prepared electrode $\left(\mathrm{rGO} / \mathrm{SnO}_{2} / \mathrm{S}\right)$ showed a discharge capacity of $575 \mathrm{~mA} \mathrm{~h} \mathrm{~g}^{-1}$ at $5 \mathrm{C}$, showing its excellent rate capability. In the same way, Wang et al. ${ }^{367}$ synthesized $\mathrm{SnO}_{2}$ nanosheets on a carbon cloth, which demonstrated a specific discharge capacity of $1228 \mathrm{~mA} \mathrm{~h} \mathrm{~g}^{-1}$ at $0.2 \mathrm{C}$ which is more than that of the sulfur cathode on a carbon cloth $\left(600 \mathrm{~mA} \mathrm{~h} \mathrm{~g}^{-1}\right.$ at $0.2 \mathrm{C}$ ). The $\mathrm{SnO}_{2} /$ carbon cloth cathode retained $76 \%$ capacity over 1000 cycles at 2C. There are reports where $\mathrm{SnO}_{2}$ was coupled with carbon nanostructures like carbon nanotubes, carbon radial nanorods, carbon hollow spheres, carbon aerogel and sulfur to form the cathode material of Li-S batteries. ${ }^{368-376}$ In all these reports, $\mathrm{SnO}_{2}$ was used to trap lithium polysulphide to mitigate the shuttling effect and ensure better utilization of active sulfur in the cathode.

\section{3 $\mathrm{SnO}_{2}$ in supercapacitors}

Supercapacitors have drawn substantial interest as an energy device among researchers, specifically due to their high power

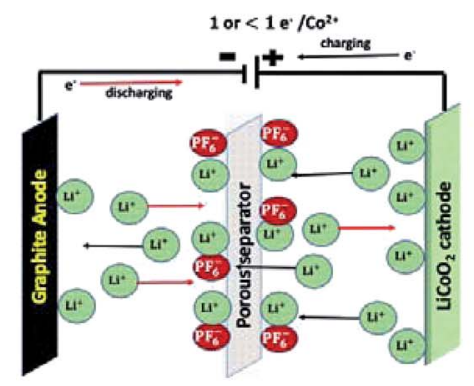

Li ion Battery

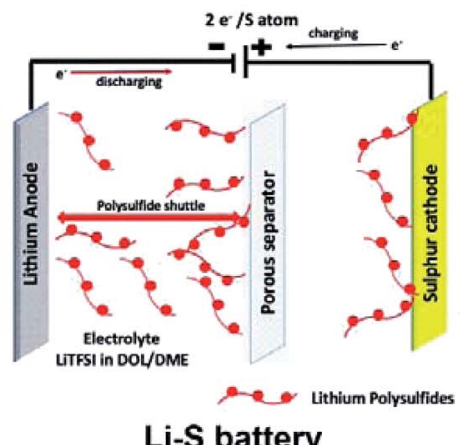

Li-S battery

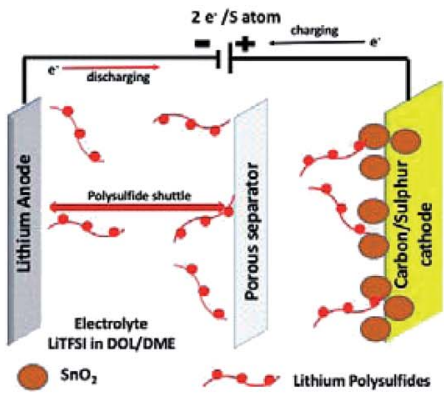

$\mathrm{SnO}_{2}$ modified Li-S battery

Fig. 31 Schematic of conventional LIBs, Li-S batteries and $\mathrm{SnO}_{2}$ modified Li-S batteries. 
(a)

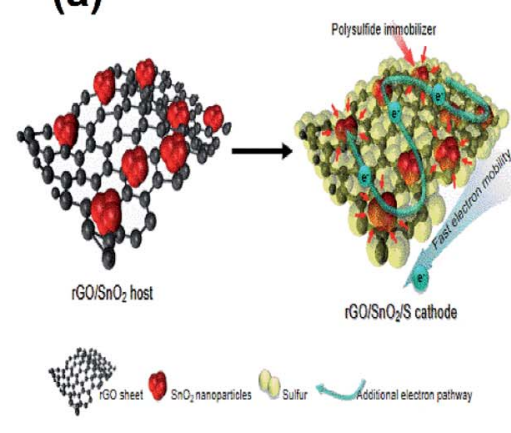

(b)

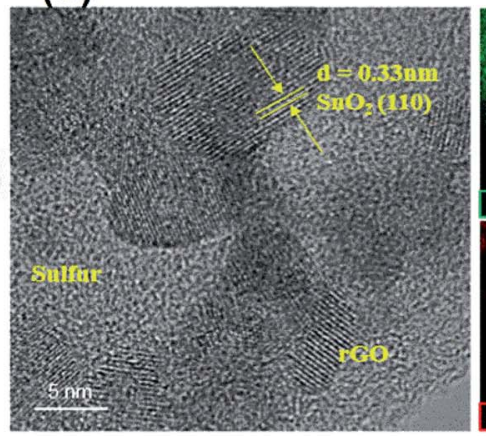

(e) (c)

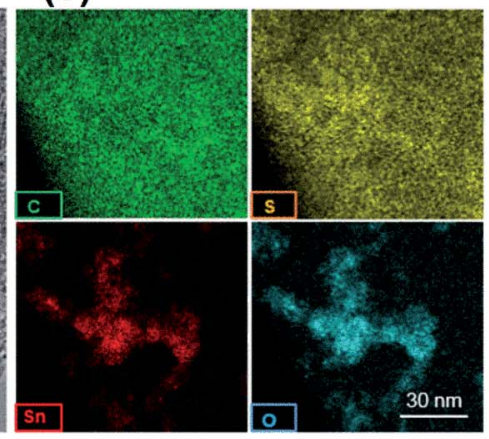

(f)
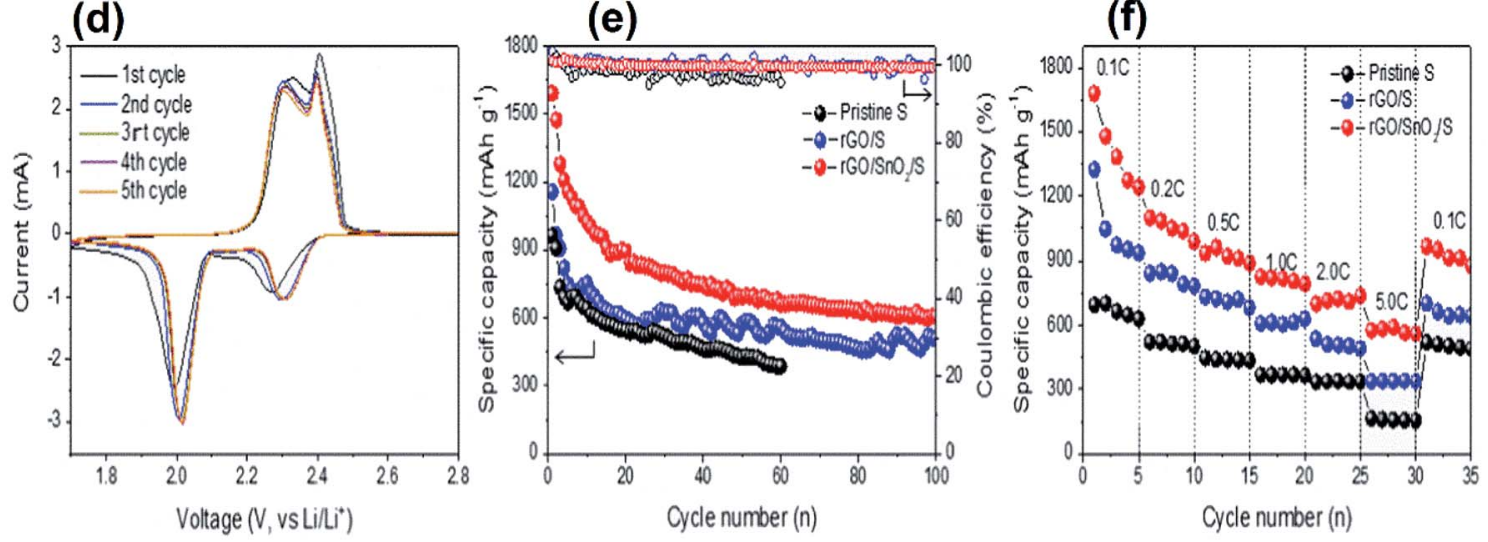

Fig. 32 Schematic representation of the $\mathrm{rGO} / \mathrm{SnO}_{2} / \mathrm{S}$ cathode acting as a polysulphide immobilizer (a); HRTEM image (b) and elemental mapping (c) of the $\mathrm{rGO} / \mathrm{SnO}_{2} / \mathrm{S}$ composite; cyclic voltammetry curve at $0.1 \mathrm{mV} \mathrm{s}^{-1}$ (d); and cycling performance (e) and rate performance (f) of the rGO/ $\mathrm{SnO}_{2} / \mathrm{S}$ composite. ${ }^{366}$ Adapted with permission of Elsevier.

density and long life cycle bridging conventional dielectric capacitors (which possess high power density) and batteries (which provide high energy density). ${ }^{377,378}$ There has been achievable progress in the design and development of electrode materials for printable/wearable electronics with enhanced capacitance and extended stability. ${ }^{379-382}$ However, advanced research is highly warranted for improving the energy density and storage capability in real-life applications such as hybrid vehicles, smartphones, and other energy storage devices in meeting the growing energy demand. ${ }^{383,384}$ The most commercially successful electrode materials in supercapacitors are carbon-supported metal/metal oxide composites. ${ }^{385,386}$ Typically, the high surface area can facilitate electrolyte ion transport onto the active sites of the electrode materials, resulting in enhancement of the electrochemical performance. Despite the advantages of carbon-based materials, electrical double-layer capacitors (EDLCs) show relatively low capacitance and limited energy density. Another strategy is to incorporate the carbon materials with metals, metal oxides, or metal-based alloys which can provide a far higher theoretical capacitance in supercapacitors. ${ }^{386,387}$ These metal/metal oxide loaded active materials in supercapacitors are commonly termed as pseudocapacitors, which can electrochemically store a large number of electrolyte ions, resulting in higher specific capacitance than EDLCs. However, the pseudo-capacitors suffer from dramatic capacitance fading in long cycles due to the reaction between the surface of active sites and electrolytes.
Recent advances in electrode materials in supercapacitors include the mixing of carbon and non-carbon-based materials to maximize the performances of these two types of materials. In this regard, hybrid electrode materials with numerous combinations involving carbon-based materials (graphene, CNTs, porous carbon, fullerenes) and metal oxides such as tin oxide ${ }^{388}$ ruthenium oxide,${ }^{389}$ manganese oxide, ${ }^{390}$ nickel-cobalt hydroxide ${ }^{391}$ cobalt oxide, ${ }^{392}$ and iron oxide ${ }^{393}$ are highly promising toward the fabrication of next-generation supercapacitors. Hence, improving the inherent materials properties by carefully fine-tuning the components involved in the electrode preparation is highly important in realizing stable and efficient energy storage materials.

Among the host of available metal oxides, ${ }^{39,216,394-400}$ tin oxide $\left(\mathrm{SnO}_{2}\right)$ is a wide-bandgap n-type $(3.6 \mathrm{eV})$ semiconducting material with excellent electrochemical properties (high theoretical capacity $\left(782 \mathrm{~mA} \mathrm{~h} \mathrm{~g}^{-1}\right)$, redox potential, good electrical conductivity $\left(21.1 \mathrm{ohm}^{-1} \mathrm{~cm}^{-1}\right)$ and thermal stability $\left(>600^{\circ} \mathrm{C}\right)$ ) leading to promising applications in a wide range of energy technologies. ${ }^{401}$ The charge storage mechanism of $\mathrm{SnO}_{2}$ as an active electrode material in an aqueous electrolyte can be written as ${ }^{402}$

$$
\mathrm{SnO}_{2}+\mathrm{H}^{+}+\mathrm{e}^{-} \leftrightarrow \mathrm{SnOOH}
$$

The utilization of nanostructured $\mathrm{SnO}_{2}$ with tailor-designed morphologies (nanocubes, nanotubes, etc.) has been studied 


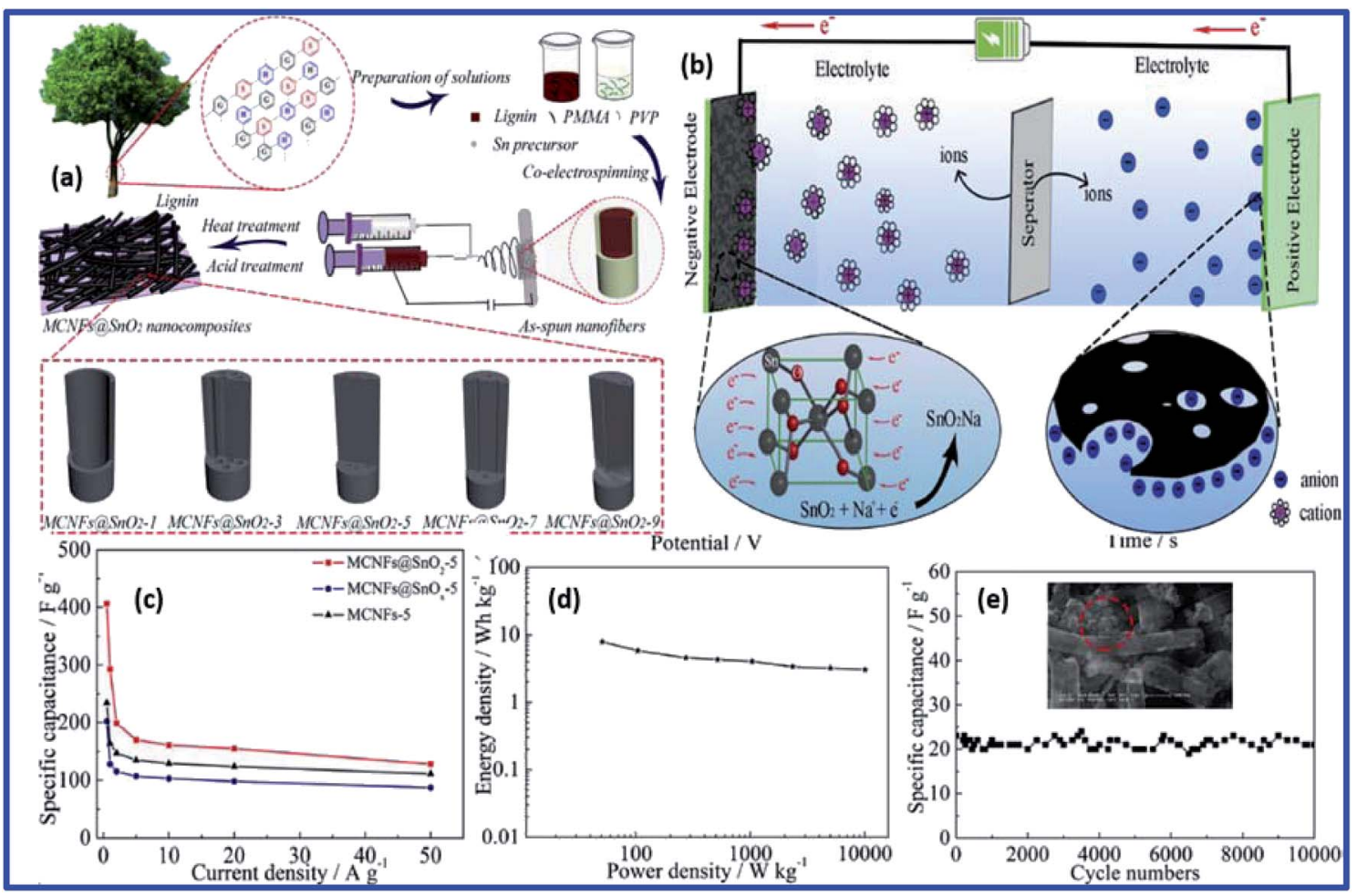

Fig. 33 (a) Fabrication steps for $\mathrm{MCNFs} \mathrm{SnNO}_{2}$, (b) proposed charge-storage mechanism, (c) current density vs. specific capacities for various

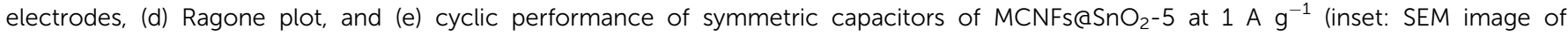
MCNFs@SnO $2-5),{ }^{406}$ presented with permission and copyright.

widely in supercapacitors. ${ }^{403-405}$ For example, nanocomposites made of a low-cost, eco-friendly carbon source (lignin-based multichannel carbon nanofibers) and $\mathrm{SnO}_{2}$ (MCNFs@SnO with varied microstructures were prepared by Han et al., through the electrospinning technique (heat-/acid-treated) and heir energy storage capability in supercapacitors was evalu-

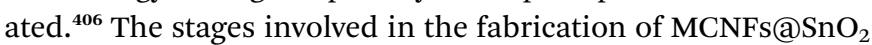
are presented in Fig. 33. A range of different electrode materials were prepared and it was found that the optimized MCNFs@SnO ${ }_{2}-5$ displayed a high specific capacitance of $128 \mathrm{~F}$ $\mathrm{g}^{-1}$ even at a high current density of $50 \mathrm{~A} \mathrm{~g}^{-1}$. It was found that the inclusion of $\mathrm{SnO}_{x}$ (after Sn washing in an acid solution) in MCNF-5 (MCNFs@SnO 2 -5) resulted in a dramatic increase in capacitance (from 202 to $406 \mathrm{~F} \mathrm{~g}^{-1}$ at $0.5 \mathrm{~A} \mathrm{~g}^{-1}$ ) with extraordinary capacity retention ( $95 \%$ after 10000 cycles, $\left.10 \mathrm{~A} \mathrm{~g}^{-1}\right)$. This remarkable improvement in the performance of MCNFs@SnO ${ }_{2}-5$ was due to the following reasons: (i) among the prepared nanocomposites, MCNFs@SnO ${ }_{2}-5$ has the largest surface area and the total pore volume of $659 \mathrm{~m}^{2} \mathrm{~g}^{-1}$ and 0.56 $\mathrm{cm}^{3} \mathrm{~g}^{-1}$ with a very narrow pore size distribution; as a result, these mesopores not only reduced the ion diffusion resistance but also improved the kinetics of the electrodes; (ii) synergistic compatibility of the ideal electrical double layer capacitor (EDLC) and pseudocapacitance components in the form of MCNFs and $\mathrm{SnO}_{2}$. The authors claim that the observed values were superior to those of previously reported doped carbon$\mathrm{SnO}_{2}$ based electrodes that include $\mathrm{N} / \mathrm{S}$ doped porous carbon nanosheets (willow catkins), ${ }^{407} \mathrm{SnO}_{2}$ nanorod/rGO ${ }^{408} \mathrm{SnS}_{2}-$
$\mathrm{SnO}_{2}$ nanocomposites, ${ }^{409} \mathrm{SnO}_{2}$ dots/activated porous carbon nanofibers, ${ }^{410} \mathrm{~N}$-doped carbon nanosheets (silk), ${ }^{411}$ etc. Carbon/ metal-oxide supported $\mathrm{SnO}_{2}$ based active materials pertaining to supercapacitors can also be found elsewhere $\left[\mathrm{ZnO} / \mathrm{SnO}_{2},{ }^{412}\right.$ $\mathrm{Co}_{3} \mathrm{O}_{4}-\mathrm{SnO} @ \mathrm{SnO}_{2},{ }^{413} \mathrm{MnO}_{2} @ \mathrm{SnO}_{2},{ }^{414} \mathrm{Ni} / \mathrm{SnO}_{2},{ }^{415}$ and graphene/ $\mathrm{SnO}_{2} /$ polypyrrole $\left.^{416}\right]$.

Several researchers utilized the combination of carbon nanofibers with heterostructures of $\mathrm{SnO}_{2}$ to tailor-design the morphology and compositional engineering through adopting various performance improvement strategies. For example, Ding et al. prepared a porous $\mathrm{CNFs} / \mathrm{SnO}_{2}$ membrane with a high specific surface area $\left(1415 \mathrm{~m}^{2} \mathrm{~g}^{-1}\right)$ through adopting sequential electrospinning, in situ-polymerization, and calcination, which displayed a high specific capacitance of $118 \mathrm{~F} \mathrm{~g}^{-1}\left(0.5 \mathrm{~A} \mathrm{~g}^{-1}\right)$ and excellent cycling durability. ${ }^{417}$ In the same way, Shao et al. fabricated heterostructured $\mathrm{CNFs} / \mathrm{SnO}_{2}$ via electrospinning and the solvothermal method and observed superior electrochemical properties due to the low resistance and rapid ion penetration. ${ }^{418}$ To overcome the conductivity issue of bare $\mathrm{SnO}_{2}{ }^{419}$ a high energy density and high rate capability flexible supercapacitor based on highly porous, low-cost, and environmentally benign materials such as additive-free 1-D $\mathrm{SnO}_{2} /$ carbon nanofibers containing self-assembled closely packed spherical uniform nanoparticles $\left(\mathrm{SnO}_{2} @ \mathrm{C}\right)$ on a carbon cloth has been designed through a facile electrospinning technique followed by calcination at different temperatures (400 and 600 $\left.{ }^{\circ} \mathrm{C}\right) .{ }^{420}$ Interestingly, the obtained energy density for $\mathrm{SnO}_{2} @ \mathrm{C}$ $\left(152 \mathrm{~W} \mathrm{~h} \mathrm{~kg}^{-1}\right)$ is much higher than that reported in other 
similar works. ${ }^{\mathbf{4 1 8 , 4 2 1}}$ Among the investigated electrode materials, $\mathrm{SnO}_{2} @ \mathrm{C}$ with $\mathrm{SnO}_{2}-600$ displayed high capacitance $\left(\sim 646 \mathrm{~F} \mathrm{~g}^{-1}\right.$ at $\left.60 \mathrm{~F} \mathrm{~g}^{-1}\right)$, energy density and power density $\left(39 \mathrm{~kW} \mathrm{~kg}^{-1}\right)$ with excellent cyclability (charge-discharge) (after 10000 cycles at a high current density of $80 \mathrm{~A} \mathrm{~g}^{-1}$ ).

Long et al. integrated a range of $\mathrm{SnO}_{2}$ dots supported and uniformly distributed activated porous carbon nanofibers (CNFs) (APCNFs) (using hard templating) on a nickel foam collector for their practical utilization in high-performance adhesive-free flexible supercapacitors, ${ }^{\mathbf{4 1 0}}$ and the electrochemical energy storage capability of bare CNFs and $\mathrm{SnO}_{2}$ was compared with that of APCNFs/SnO ${ }_{2}$. The inclusion of $\mathrm{SnO}_{2}$ dramatically improved the electrochemical performance of the resultant electrode, APCNFs/SnO effect between $\mathrm{SnO}_{2}$ and APCNFs, offering fast electron transfer and ion penetration during charge/discharge processes. The asconstructed electrode displayed high energy and power densities of $10.3 \mathrm{~W} \mathrm{~h} \mathrm{~kg}^{-1}$ and $325 \mathrm{~W} \mathrm{~kg}^{-1}$, respectively, which are sufficient enough to light a red-color indicator connected in series of $1 \times 1 \mathrm{~cm}^{2}$ (asymmetric) with high tensile stress and better mechanical strength. Even after testing at a high bending angle $\left(45\right.$ to $\left.180^{\circ}\right)$, negligible degradation was observed (Fig. 34).

In another interesting report, Amreesh Chandra et al. devised a universal strategy and proposed a growth mechanism (solid/hollow) of hollow structured metal oxides (simple binary to complex ternary oxides as well) as an emerging electrode material for next-generation supercapacitors. ${ }^{422}$ The authors have prepared a range of metal oxide nanostructures that include $\mathrm{SnO}_{2}, \mathrm{Cu}_{2} \mathrm{O}, \mathrm{Co}_{3} \mathrm{O}_{4}$, and $\mathrm{Fe}_{2} \mathrm{O}_{3}$, among others, and found that the unit cell parameters do not differ for solid and hollow type nanoparticles. Subsequently, the as-developed solid and hollow types of metal oxides were tested for supercapacitors through conventional electrochemical measurements. Hollow structuring of metal oxides displays much improved electrochemical performance (close to 2-fold) primarily due to increased redox activity and surface adsorption active sites. The authors envisage that this type of nanostructuring will outperform other similar energy storage systems (electrode materials based on graphene, conducting polymers, etc.).

The combination of electrochromism and supercapacitors offers a new class of devices referred to as electrochromic supercapacitors (ECSs), which can directly indicate their energy storage level through changes in color. This feature allows users to straightforwardly determine the real-time status of the device without using additional instruments and provides visual warnings to prevent overcharging. Electrochromic (EC) chromophores, the core materials of ECSs, chemically store injected charges via electrochemical charging/discharging processes. Simultaneously, optical modulation occurs through electrochromic transitions. As an electrochromic supercapacitor material, tungsten oxide $\left(\mathrm{WO}_{3}\right)$ is most extensively studied. ${ }^{\mathbf{4 2 3 - 4 2 6}}$ However, in all these reports, FTO has been used as the TCO. In a recent study by Jo et al., ${ }^{\mathbf{4 2 7}}$ the authors had demonstrated a novel method of increasing the F doping content in FTO films and its application in the field of electrochromic energy storage devices. The doping content of $\mathrm{F}$ was increased by varying sodium hydroxide $(\mathrm{NaOH})$, which acted as a functional additive to the precursor solution during spray pyrolysis. An increase in the F-doping concentration in $\mathrm{NaOH}$ modified FTO films enhanced the carrier concentration as well as surface

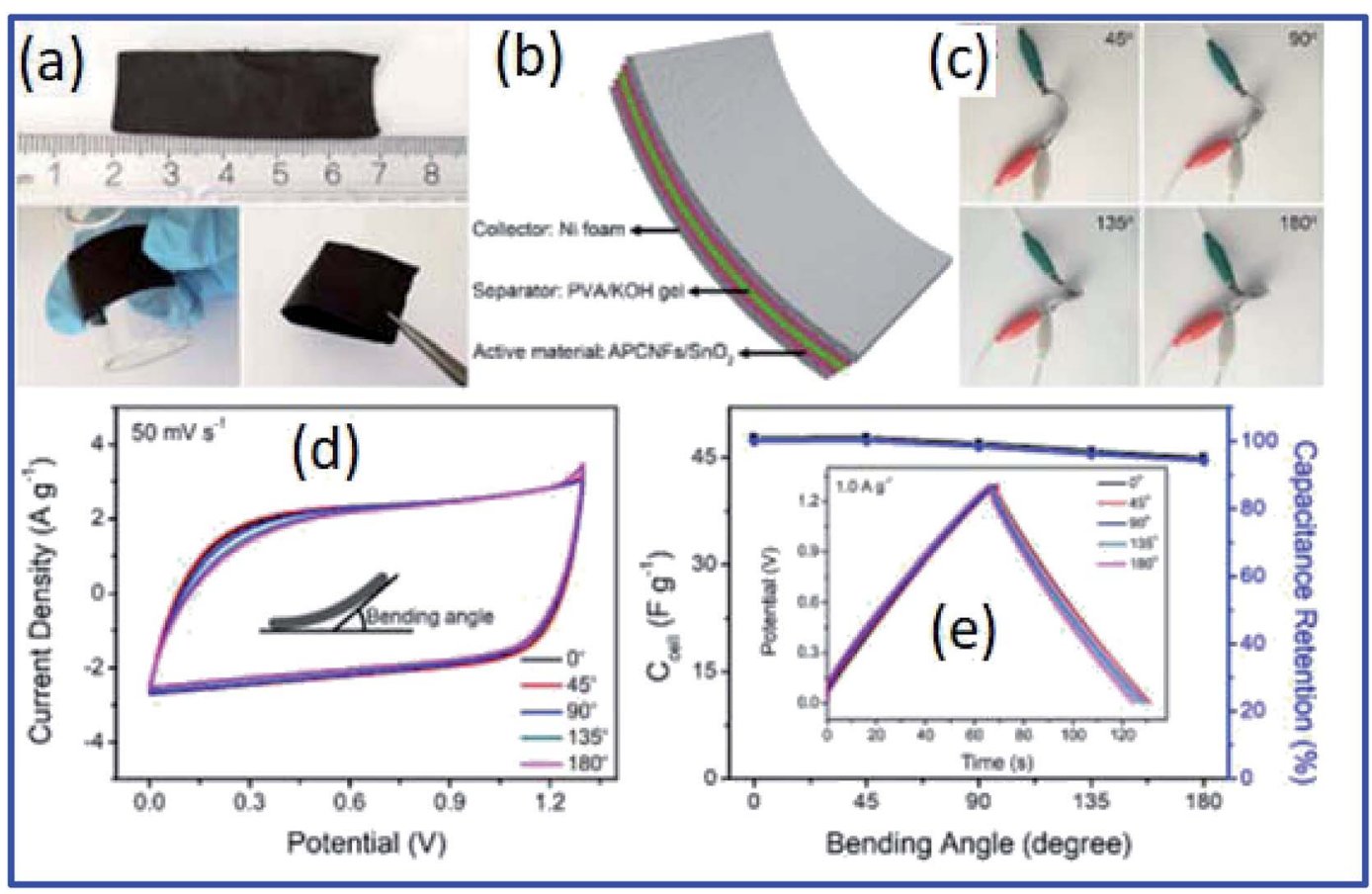

Fig. 34 (a) Flexible APCNFs/SnO 2 membrane, (b) sandwich-type symmetric cell, (c) optical image, (d) CV curves and (e) specific capacitance and capacity retention at various bending angles (inset: their corresponding galvanostatic charge-discharge profiles), ${ }^{410}$ presented with permission and copyright. 
densification that was beneficial to Hall mobility, resulting in a lower sheet resistance $\left(R_{\mathrm{sh}}\right)$ of the $\mathrm{NaOH}$ modified FTO films. As a result of the improved conduction in the $\mathrm{NaOH}$ modified FTO films fabricated with the volume percentage of $5 \mathrm{vol} \%$, the films showed enhanced electrochromic (EC) performances, fast switching speeds (6.6 $\mathrm{s}$ for coloration speed and $5.4 \mathrm{~s}$ for bleaching speed) and a superb coloration efficiency (CE) of 58.1 $\mathrm{cm}^{2} \mathrm{C}^{-1}$. The fast-switching speeds were caused by the accelerated $\mathrm{Li}^{+}$and electron transport in the active electrodes due to the decreased $R_{\mathrm{sh}}$ and a superb CE value was generated from the broadened $\Delta T$ as an effect of increased electrochemical activity. In addition to EC performance, the enhanced electrochemical activity induced by the decreased $R_{\mathrm{sh}}$ of modified FTO films resulted in efficient transport of a large quantity of $\mathrm{Li}^{+}$and electrons into the active electrodes, bringing about improved energy storage performance, e.g. a higher specific capacitance
(65.2 $\mathrm{F} \mathrm{g}^{-1}$ at $2 \mathrm{~A} \mathrm{~g}^{-1}$ ), which was clearly evident from the $\mathrm{CV}$, charge-discharge and EIS profiles of $\mathrm{WO}_{3}$ nanostructures on both bare FTO and $\mathrm{NaOH}$ modified FTO films.

The electrochromic properties of $\mathrm{SnO}_{2}$ have been well known since the early 2000s. ${ }^{\mathbf{4 2 8 - 4 3 1}}$ There are reports on the electrochromic performance of not only $\mathrm{SnO}_{2}$ nanostructures ${ }^{\mathbf{4 3 1}}$ but also the doped $\mathrm{SnO}_{2}$ (ref. 430 and 431) and $\mathrm{SnO}_{2}$ based heterostructures with other metal oxides like $\mathrm{NiO}, \mathrm{WO}_{3}$, and $\mathrm{TiO}_{2}{ }^{428,429,432,433}$ However, it came to our notice that the transition metal oxides like $\mathrm{NiO}, \mathrm{TiO}_{2}$, and $\mathrm{WO}_{3}$ used to design heterostructures along with $\mathrm{SnO}_{2}$ to study the electrochromic performance of the heterostructures are promising supercapacitor electrode materials. Despite the existence of these heterostructures, the field of electrochromic energy storage devices is mostly dominated by $\mathrm{WO}_{3}$ based electrodes. Thus, these heterostructures can be more efficient than $\mathrm{WO}_{3}$

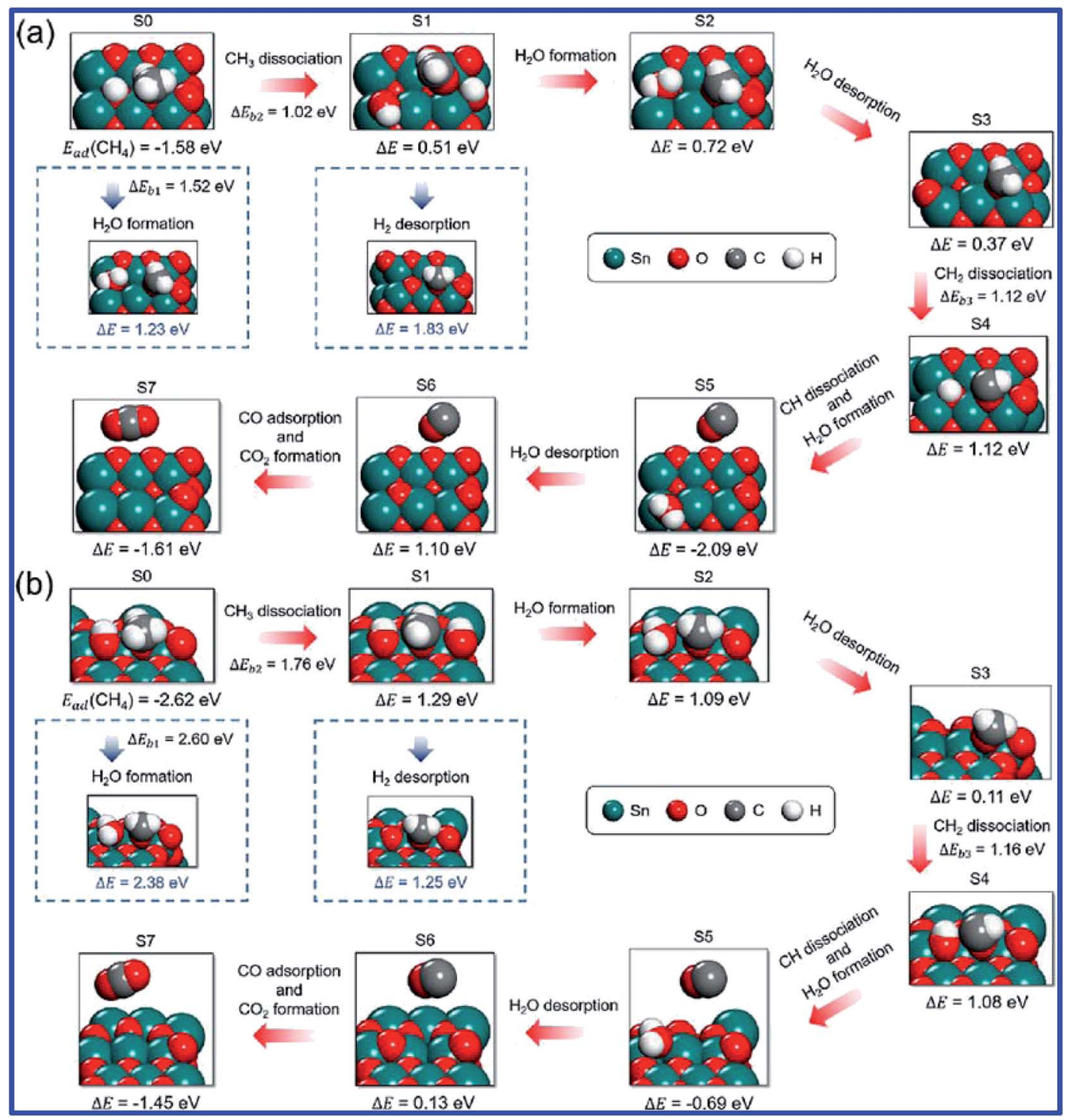

Fig. 35 Energetics of $\mathrm{SnO}_{2}$ reduction by surface-bound $\mathrm{CH}_{4}$. (a) $\mathrm{SnO}_{2}(100)$ surface. (b) $\mathrm{SnO}_{2}$ (110) surface. On both $\mathrm{SnO} 2$ facets, the formation of $\mathrm{H}_{2} \mathrm{O}$ was energetically preferred to $\mathrm{H}_{2}$. The red arrows present the preferred reaction pathway. $E_{a d}\left(\mathrm{CH}_{4}\right)$ represents the adsorption energy of $\mathrm{CH}_{4}$ on the $\mathrm{SnO}_{2}$ surfaces. $\Delta E$ of each step represents the energetic state of the current state relative to the previous state. For example, $\Delta E=$ $0.51 \mathrm{eV}$ of $\mathrm{S} 1 \mathrm{in}(\mathrm{a})$ means that $0.51 \mathrm{eV}$ of energy is required for $\mathrm{CH}_{3}$ dissociation from S0 to $\mathrm{S}_{1},{ }^{434}$ presented with permission and copyright. 
nanostructures because of their superior pseudocapacitive behavior, and these heterostructures will be crucial in developing the electrodes for electrochromic energy storage devices.

\section{9. $\mathrm{SnO}_{2}$ in circular Economies}

Tin has been in use since ancient times, and it has played an important role in the history of the technological race. Over the centuries, tin has become an indispensable ingredient in modern life, supported by mobile devices, solar cells, and wearable devices. The worldwide tin consumption reached 400000 tons per year, powered by a global boom in consumer electronics and a rapid transition to the use of lead-free solders. Hence, in recent years, recycling methods of tin are on the rise. Nearly $30 \%$ of the total tin used is derived from electronic waste or e-waste. It is also called 'urban mining' and has gained momentum in different countries, thus generating 70000 tons of recycled tin. The circular economy is a way towards the management of waste and pollution, keeping materials in use and regenerating natural systems. It engages a closed loop system, which involves recycling, sharing, repair, refurbishment, and remanufacturing, to reduce the resource usage, waste creation and carbon footprint. The recovery of $\mathrm{Sn}$ or $\mathrm{SnO}_{2}$ by a novel $\mathrm{CH}_{4}$ reduction method has been reported using thermodynamic simulations and density functional theory. ${ }^{434}$ The experiment suggests that $\mathrm{CH}_{4}$ actively reduces $\mathrm{SnO}_{2}$ at $1000{ }^{\circ} \mathrm{C}$ to produce high purity Sn. The reported reduction method supports a circular economy through a cost-effective recycling strategy. Fig. 35 shows the mechanism involving the energetics of $\mathrm{SnO}_{2}$ reduction by surface-bound $\mathrm{CH}_{4}$. The method suggests the efficient and economic recovery of a metal element from metal oxide waste.

In another report, the synthesis of cambered nano-walls of $\mathrm{SnO}_{2} / \mathrm{rGO}$ materials using a melamine template has been reported (Fig. 36). Melamine acts as an ideal recyclable template due to its good adsorbent capacity for $\mathrm{M}(x)-\mathrm{SnO}_{2} / \mathrm{rGO}$ and precipitation after cooling.

The thickness of $\mathrm{SnO}_{2} / \mathrm{rGO}$ nano-walls was controlled by varying the melamine content. The synthesized $\mathrm{SnO}_{2} / \mathrm{rGO}$ showed a reversible capacity of $-998 \mathrm{~mA} \mathrm{~h} \mathrm{~g}^{-1}$ at a current density of $100 \mathrm{~mA} \mathrm{~g}^{-1}$ and a capacity of $855 \mathrm{~mA} \mathrm{~h} \mathrm{~g}^{-1}$ in a 0.02 to $3.0 \mathrm{~V}$ potential $v s$. $\mathrm{Li}^{-\mathrm{Li}^{+}}$. Cambered nano-walls facilitated fast solid diffusion and an efficient liquid channel to improve the reversible capacity. The method is scalable and applicable to other graphene-based energy materials. ${ }^{435}$ The synthesis route is easily scalable, cost-effective, environmentally friendly, and energy-efficient. Resource-saving, economic and eco-friendly recycling of $\mathrm{SnO}_{2} / \mathrm{Sn}_{3} \mathrm{O}_{4}$ from $\mathrm{Sn}$ anode slime for the development of gas sensing materials is illustrated in Fig. $37 .{ }^{436} \mathrm{Sn}$ anode slime, a by-product of Sn plating, has gas sensing capabilities. Ag-doped $\mathrm{SnO}_{2} / \mathrm{Sn}_{3} \mathrm{O}_{4}$ as a sensing material for formaldehyde gas was synthesized using the dipping route. At $2 \mathrm{~mol} \%$ of $\mathrm{Ag} / \mathrm{Ag}_{2} \mathrm{O}$-doped $\mathrm{SnO}_{2} / \mathrm{Sn}_{3} \mathrm{O}_{4}$, good sensing properties were observed at a high response of 12.76 to $100 \mathrm{ppm}$ formaldehyde at $160{ }^{\circ} \mathrm{C}$ temperature. Fig. 37 illustrates that more oxygen molecules are adsorbed on $\mathrm{SnO}_{2} / \mathrm{Sn}_{3} \mathrm{O}_{4}$; consequently, more electrons can be trapped from the conduction band after the Ag decoration. The decremented free-electron concentration forms a thicker electron depletion region with a higher electrical resistance state compared to the unmodified base material. $\mathrm{Ag} / \mathrm{Ag}_{2} \mathrm{O}$ allows more adsorption of formaldehyde molecules to react with adsorbed oxygen species. The work has demonstrated an eco-process of recycling tin oxides from tinbased anode slime as a formaldehyde gas sensitive material.

\section{Comparison of Ga-doped $\mathrm{ZnO}$ and $\mathrm{SnO}_{2}$ as TCOs}

Although this review article is focused on doped $\mathrm{SnO}_{2}$ for TCO applications, the reader should be aware that there are two other important categories of doped metal oxide TCOs, namely $\mathrm{In}_{2} \mathrm{O}_{3}$ and $\mathrm{ZnO}^{116}$ The most commonly used TCO material, ITO, belongs to the former category. Although it is often referred to as Sn doped indium oxide, it is actually a mixed compound of $\mathrm{In}_{2} \mathrm{O}_{3}$ and $\mathrm{SnO}_{2}$ with the former being the dominant component. Given the limited global geological reserves of indium of $\sim 6000$ tonnes, ${ }^{116}$ it is essential to develop alternative sustainable TCOs for optoelectronic as well as energy devices. Doped $\mathrm{ZnO}$ is a more recent and promising TCO material that offers a similar performance with respect to doped $\mathrm{SnO}_{2}$. Research on ZnO based TCO began during the 1990s. Since about 2010, the ZnO based TCO has been recognized as a viable low-cost alternative to ITO, especially for thin film photovoltaic devices. In

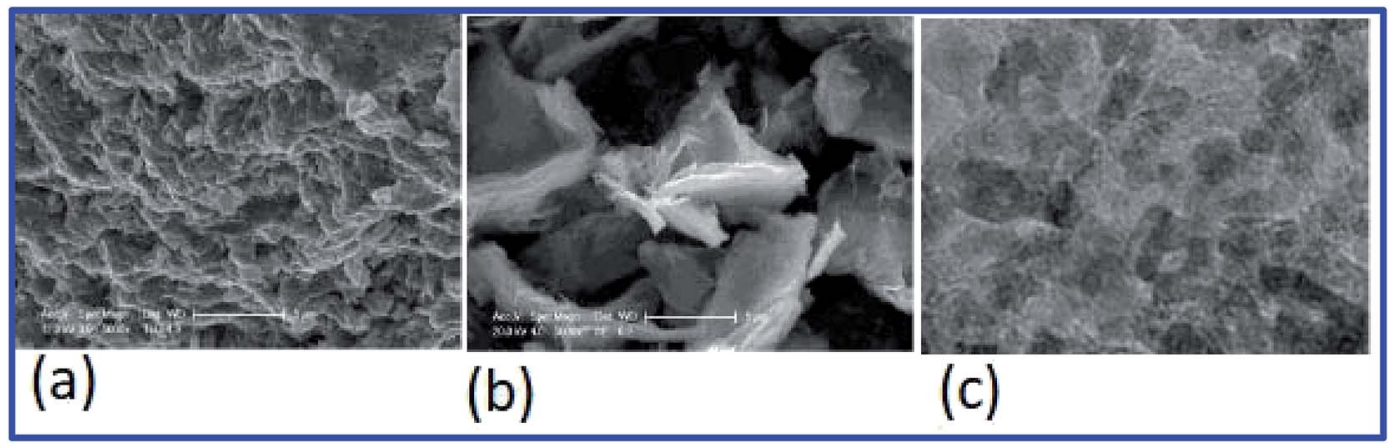

Fig. $36 \mathrm{SEM}$ images of (a) $\mathrm{SnO}_{2} / \mathrm{rGO}$ and (b) $\mathrm{M}(2)-\mathrm{SnO}_{2} / \mathrm{rGO}$. (c) HRTEM image, ${ }^{435}$ presented with permission and copyright. 


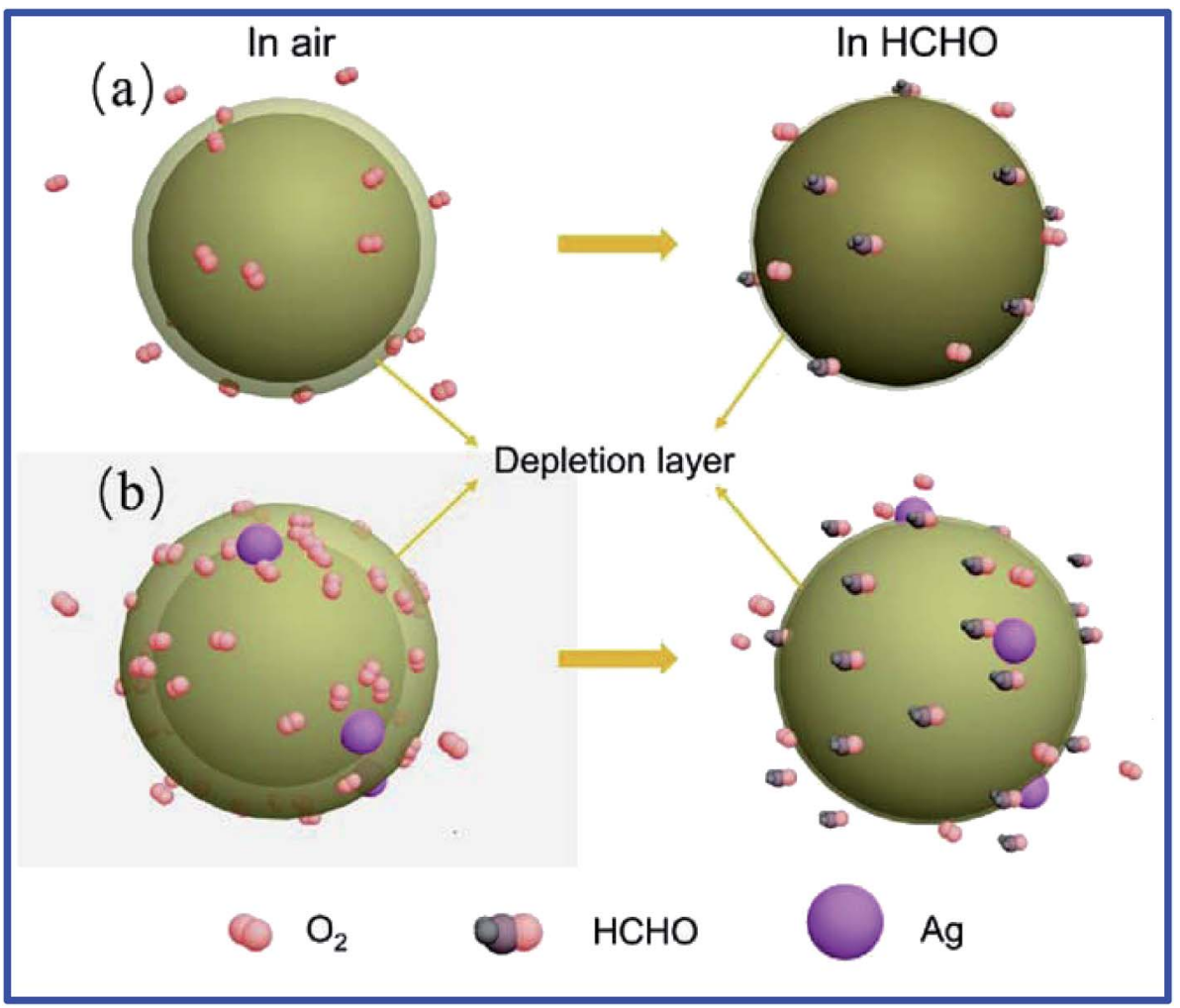

Fig. 37 Schematic model of the depletion layer for $\mathrm{SnO}_{2} / \mathrm{Sn}_{3} \mathrm{O}_{4}$ materials when exposed to air and $\mathrm{HCHO}$ (a) before Ag decoration and (b) after Ag decoration, ${ }^{436}$ presented with permission and copyright.

this section, ZnO based TCOs will be briefly compared with those based on $\mathrm{SnO}_{2}$ to provide a more balanced perspective.

$\mathrm{ZnO}$ is a wide bandgap semiconductor with a hexagonal wurtzite crystal structure and a direct energy bandgap of $3.28 \mathrm{eV} .{ }^{437}$ The $\mathrm{ZnO}$ band gap is wider than that of $\operatorname{In}_{2} \mathrm{O}_{3}(2.9 \mathrm{eV})$ but narrower than that of $\mathrm{SnO}_{2}(3.62 \mathrm{eV}) .{ }^{\mathbf{1 1 6}}$ The electron affinity of $\mathrm{ZnO}$ is $4.35 \mathrm{eV}$. Like $\mathrm{SnO}_{2}$, undoped $\mathrm{ZnO}$ is weakly n-type with a typical carrier (electron) concentration of $\sim 10^{17} \mathrm{~cm}^{-3}$. The carrier concentration and the electrical conductivity of $\mathrm{ZnO}$ can be increased by doping with a wide variety of n-type dopants such as: $\mathrm{Ga}, \mathrm{Al}, \mathrm{B}, \mathrm{In}, \mathrm{Si}, \mathrm{Ge}, \mathrm{Ti}$ and $\mathrm{F} .{ }^{\mathbf{1 8 0}}$ These dopants donate electrons to the conduction band of $\mathrm{ZnO}$ by substituting $\mathrm{Zn}^{2+}$ ions in the $\mathrm{ZnO}$ lattice. Amongst the above dopants, $\mathrm{Ga}$ and $\mathrm{Al}$ are the more widely studied and they are also commercially important. ${ }^{438}$ For brevity, we focus on TCO films based on Ga doped $\mathrm{ZnO}$ (GZO). There has been much interest in GZO as

Table 6 Deposition and properties of GZO TCO films

\begin{tabular}{|c|c|c|c|c|c|c|}
\hline Deposition technique & $\begin{array}{l}\text { Process } \\
\text { temperature }\left({ }^{\circ} \mathrm{C}\right)\end{array}$ & Thickness (nm) & Resistivity $(\Omega \mathrm{cm})$ & $\begin{array}{l}\text { Average } \\
\text { transmittance (\%) }\end{array}$ & $\begin{array}{l}\text { Mobility } \\
\left(\mathrm{cm}^{2} \mathrm{~V}^{-1} \mathrm{~s}^{-1}\right)\end{array}$ & Reference \\
\hline Spray pyrolysis & $350-500$ & $\sim 350$ & $4.9 \times 10^{-2}$ & 85 & - & Tiburcio-Silver et al. ${ }^{451}$ \\
\hline RF sputtering & $100-300$ & - & $2.2 \times 10^{-4}$ & 85.9 & $10-30$ & Miyazaki et al. ${ }^{450}$ \\
\hline RF sputtering & RT & $600-800$ & $7.8 \times 10^{-4}$ & 80 & 14.1 & Gong et al. ${ }^{449}$ \\
\hline Chemical spray & $425-525$ & 600 & $7.4 \times 10^{-3}$ & 80 & $0.1-1.0$ & Gómez et al. ${ }^{448}$ \\
\hline Sol-gel & 500 & 65 & 280 & $91 / 5$ & - & Tsay et al. ${ }^{452}$ \\
\hline Spray pyrolysis & 350 & 200 & $6.8 \times 10^{-3}$ & $\sim 90$ & 29.2 & Rao et al. ${ }^{447}$ \\
\hline RF sputtering & $40-60$ & $50-80$ & $3.1 \times 10^{-4}$ & 83 & $3-12$ & Yu et al. ${ }^{446}$ \\
\hline RF sputtering & RT & 600 & $2.6 \times 10^{-4}$ & 90 & $1-10$ & Assunção et al. ${ }^{445}$ \\
\hline DC reactive sputtering & 300 & 500 & $3.51 \times 10^{-4}$ & 90 & $4-8$ & Ma et al. ${ }^{444}$ \\
\hline PLD & $100-500$ & 200 & $8.12 \times 10^{-5}$ & 90 & $19.12-30.96$ & Park et al. ${ }^{443}$ \\
\hline PLD & RT to 500 & $200-300$ & $5.96 \times 10^{-4}$ & 92 & $0.1-10$ & Vincze et $a l . .^{442}$ \\
\hline ALD & $200-300$ & $60-70$ & $4 \times 10^{-4}$ & 95 & $6.4-20$ & Szabó et al. ${ }^{441}$ \\
\hline Sol-gel & $300-500$ & - & $4.6 \times 10^{-3}$ & - & $6.7-9.78$ & Al-Asedy et al. ${ }^{440}$ \\
\hline Aerosol assisted CVD & 450 & $400-600$ & $7.8 \times 10^{-4}$ & $>80$ & $7.7-15.1$ & Ponja et al. ${ }^{439}$ \\
\hline
\end{tabular}


a viable alternative to ITO and doped $\mathrm{SnO}_{2}$ because $\mathrm{GZO}$ is a low cost, earth abundant material that is non-toxic and sustainable. Table 6, which has a structure similar to those of Tables 1 and 2, summarizes the deposition processes and properties of GZO films, as reported in the literature over the period 19972020.439-452 Similar to doped $\mathrm{SnO}_{2}$, GZO can be deposited by a variety of solution processing and physical vapor deposition techniques. The main solution processing techniques for GZO deposition are sol-gel spin coating and spray pyrolysis (or chemical spray). ${ }^{\mathbf{4 0 , 4 4 8 , 4 5 1 , 4 5 2}}$ For physical vapor deposition of GZO, the main techniques used are RF magnetron sputter-

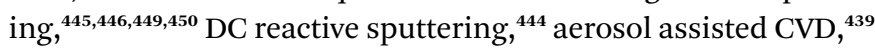

ALD $^{\mathbf{4 4 1}}$ and PLD. ${ }^{\mathbf{4 2 , 4 4 3}}$ Although not explicitly stated, the typical Ga dopant concentration used during deposition is in the range of 1-9 at $\%$ for the cited references in Table $6 .^{\mathbf{4 3 9 - 4 5 2}}$ The process temperature column refers to the highest processing temperature for the deposition techniques shown. It can be seen that for most of these techniques, deposition of GZO can be carried out by using mild to moderate substrate heating $\left(40-400{ }^{\circ} \mathrm{C}\right)$ and room temperature deposition is possible for RF sputtering. ${ }^{\mathbf{4 4 9}}$ This is especially important for the deposition of TCOs for thin film photovoltaic devices fabricated on flexible polymeric substrates or substrates with low melting points.

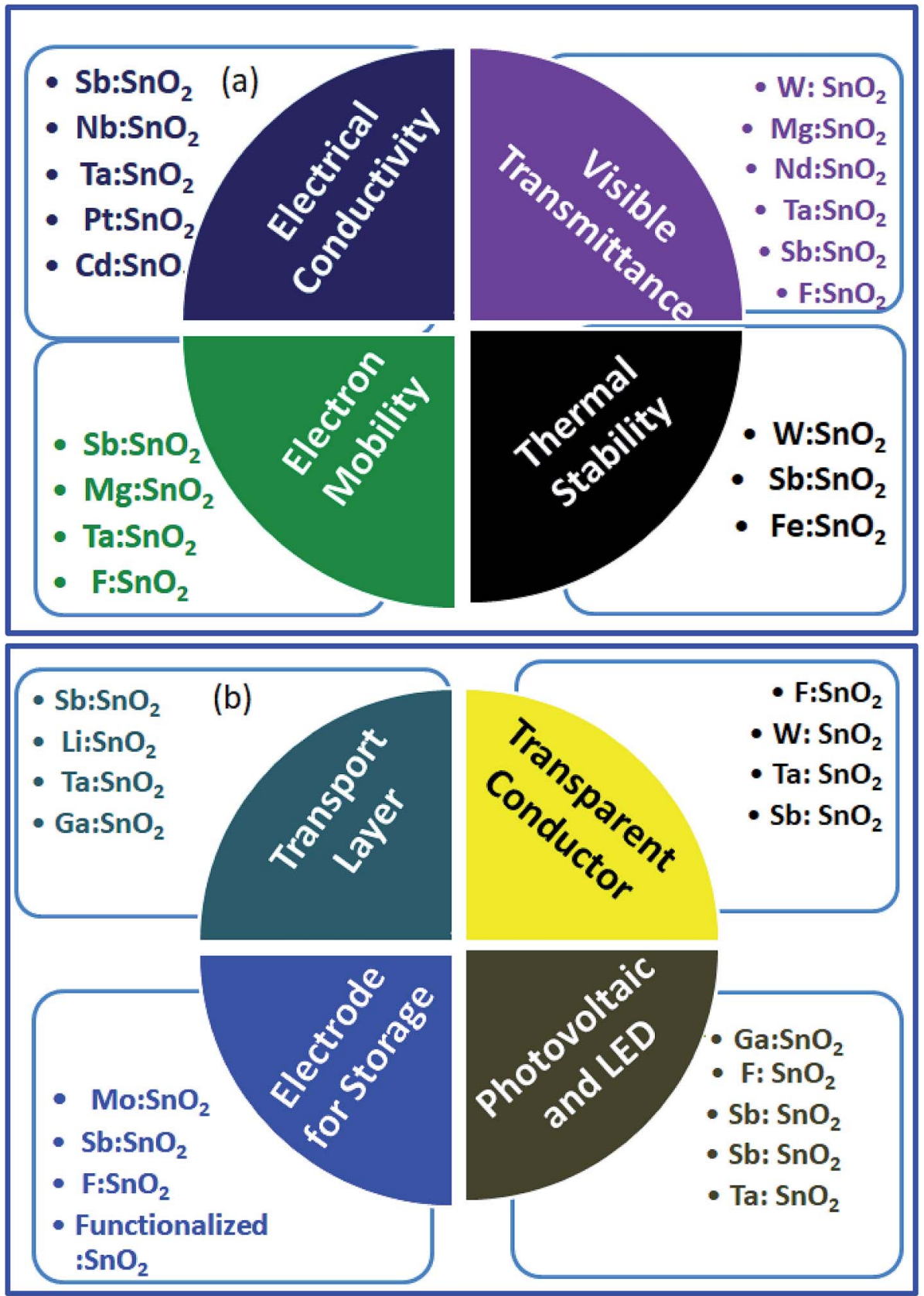

Fig. 38 (a) Selection of dopants for high electrical conductivity, visible transmittance, carrier mobility and thermal stability. (b) Selection of dopants for different applications. 
The resistivity of GZO films shown in Table 6 is the minimum resistivity reported in each publication. These are generally within the range of $1 \times 10^{-4}$ to $5 \times 10^{-2} \Omega \mathrm{cm}$ and are comparable to the resistivity of doped $\mathrm{SnO}_{2}$ tabulated in Tables 1 and 2. The low resistivity for GZO is partly due to a high carrier concentration in the range of $10^{20}-10^{21} \mathrm{~cm}^{-3} \cdot{ }^{8}$ The PLD technique in particular can yield the lowest GZO resistivity of $8.12 \times$ $10^{-5} \Omega \mathrm{cm}$, which is comparable to the lowest reported resistivity of $\sim 7.7 \times 10^{-5} \Omega \mathrm{cm}$ for ITO. ${ }^{453}$ Note that both solution processing and physical vapor deposition techniques can yield GZO films with resistivity within this range of $1 \times 10^{-4}$ to $5 \times$ $10^{-2} \Omega \mathrm{cm}$, which is a narrower range than that of $\mathrm{SnO}_{2}$ in Tables 1 and 2 .

As for optical properties, the average transmittance of GZO films in the visible range in Table 6 is consistent in the range of 80-90\% for GZO deposited by solution processing and physical vapor deposition techniques. The average transmittance of GZO films is comparable to that of doped $\mathrm{SnO}_{2}$ films deposited by physical vapor deposition, as shown in Table 2. The Hall mobility of GZO films shown in Table 6 is generally in the range of $1-30 \mathrm{~cm}^{2} \mathrm{~V}^{-1} \mathrm{~s}^{-1}$. This is again comparable to the mobility of doped $\mathrm{SnO}_{2}$ films shown in Tables 1 and 2 .

In addition to the properties shown in Table 6, GZO is known to be chemically stable at elevated temperatures, ${ }^{454}$ and the electrical resistivity of some doped $\mathrm{ZnO}$ is not a sensitive function of temperature. ${ }^{455}$ Both these properties are advantageous from a device fabrication standpoint. Surface morphology is another critically important property of TCOs because the surface roughness can cause pinholes in film layers deposited over the TCO. For GZO, it has been found that increasing the Ga dopant concentration in $\mathrm{ZnO}$ can enhance the surface diffusion and both the crystallite size and RMS surface roughness are reduced. ${ }^{456} \mathrm{~A} 1.17 \mathrm{~nm}$ RMS surface roughness has been observed for sol-gel derived GZO with 5 at\% Ga. ${ }^{452}$ The comparison of materials data listed in Tables 1, 2 and 6 shows that GZO is an equally suitable TCO thin film material as doped $\mathrm{SnO}_{2}$. One known drawback of $\mathrm{ZnO}$ based TCO, however, is its vulnerability to damp heat. ${ }^{\mathbf{1 1 6}}$ Under direct exposure to damp heat at $85{ }^{\circ} \mathrm{C}$ and high relative humidity of $85 \%$, the film properties of $\mathrm{Al}$ doped $\mathrm{ZnO}$ (AZO) have been observed to deteriorate rapidly. ${ }^{\mathbf{1 1 6}}$ For transparent flexible electronics, there may be an advantage in using GZO because $\mathrm{ZnO}$ is also an important transparent semiconducting oxide for the channel layer of thin film transistors (TFTs). ${ }^{457} \mathrm{~A}$ TFT is often used as the pixel switching device for the backplane circuit of active matrix displays and for flexible electronic circuits. This allows the same metal oxide material to be used for the channel and the substrate.

The present review emphasizes on enhancing the electrical and optical performances of $\mathrm{SnO}_{2}$ by designing suitable dopants, synthesis approaches via physical and chemical methods, formation of a multilayer structure with metals, and functionalization of the $\mathrm{SnO}_{2}$ layer. The high visible transmittance, electrical conductivity and bulk carrier mobility can be tuned precisely by selecting the dopants and synthesis process. Fig. 38a depicts the mapping of dopants to achieve high electrical conductivity, visible transmittance, electron

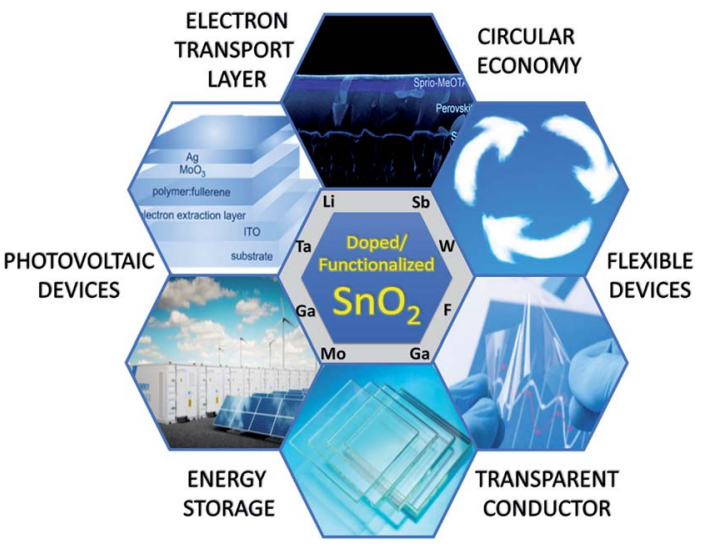

Fig. 39 Doped $\mathrm{SnO}_{2}$ for various applications.

mobility and thermal stability. It is also worth noting that the device performance significantly depends on the design of the dopants in $\mathrm{SnO}_{2}$ (Fig. 38b).

The review has been designed from the perspective of basic information about $\mathrm{SnO}_{2}$ to the advance development of $\mathrm{SnO}_{2}$ through different processes, structuring, and dopant design. We have emphasized on the synthesis of $\mathrm{SnO}_{2}$ using the sol-gel method, spray coating, chemical bath deposition, sputter deposition technique and CVD at the industrial level, for large scale deployment of $\mathrm{SnO}_{2}$ based photovoltaic devices and energy storage devices. Furthermore, since $\mathrm{SnO}_{2}$ plays a critical role as the charge transport layer, this review maps the dopants of $\mathrm{SnO}_{2}$ for various applications (Fig. 39) and studied in detail the impact of a $\mathrm{SnO}_{2}$ based ETL for photovoltaic, LED, and wearable devices towards designing stable and efficient organic/ inorganic and perovskite-based solar cells and LEDs.

\section{Conclusions and future perspectives}

Doped $\mathrm{SnO}_{2}$ is a promising non-toxic earth-abundant TCO that can be synthesized by using both solution and vacuum-based deposition techniques. Different types of cationic and anionic dopants such as Sb, Nb, Co, Ga, Al, Ta, W, F, Li, Mo, and $\mathrm{N}$ have been discussed in detail. The substitution of $\mathrm{Sn}^{4+}$ or $\mathrm{O}^{2-}$ ions by these dopant elements modifies the electronic structure of $\mathrm{SnO}_{2}$ and results in changes of material properties. $\mathrm{Sb}$ and $\mathrm{F}$ are the most suitable dopants for $\mathrm{SnO}_{2}$ because both ATO and FTO can simultaneously achieve desired properties in transparency, resistivity, mechanical hardness, environmental stability, and Hall mobility. In the case of $\mathrm{NBO}$, the radii of $\mathrm{Nb}$ and $\mathrm{Sn}$ are closer than that of F, resulting in higher conductivity than FTO. Ga-doped $\mathrm{SnO}_{2}$ is an efficient electron transport material in planar perovskite solar cells. In addition, Al- and-Ga doped tin oxide can convert the conductivity type from n-type (pure $\mathrm{SnO}_{2}$ ) to p-type. Ta-doped $\mathrm{SnO}_{2}$ epitaxial films show excellent Hall mobility of $\sim 58 \mathrm{~cm}^{2} \mathrm{~V}^{-1} \mathrm{~s}^{-1}$ and transparency extended to the UV-B spectral region (280-315 $\mathrm{nm}$ ), which enables applications in transparent electric and photoelectric devices. 
Apart from dopant categories, the surface roughness, film thickness and thermal treatment have significant impacts on the electrical and optical properties of optoelectronics devices. Smoother surfaces reduce the contact resistance and localized field effects. On the other hand, intentionally designed rough or patterned surfaces trap incident light through increasing the optical path length of the light, thereby increasing the amount of light absorbed by the active layers. A pyramidal surface is found to have a larger transmission and efficiency than a planar surface.

The structural, optical, and electronic properties of doped $\mathrm{SnO}_{2}$ significantly depend on the growth procedure and postdeposition thermal treatment. Furthermore, the temperature gradient from the surface of the film to the substrate can limit impurity diffusion from the substrate. For the $\mathrm{SnO}_{2} /$ metal based multilayer thin films, which also show high conductivity and visible transmittance, a grid structure fabricated by the lift-off process can further enhance the visible transmittance without adversely affecting the electrical properties of the multilayer.

Thin-film $\mathrm{SnO}_{2}$ can also act as an ETL on FTO substrates. Doped $\mathrm{SnO}_{2}$ can enhance the performance of OLED and photovoltaic devices by facilitating the electron injection and extraction, respectively, through improved alignment of the conduction band edge with the metal Fermi level. For perovskite thin film solar cells, annealing the $\mathrm{SnO}_{2}$ ETL in the range of $100{ }^{\circ} \mathrm{C}$ to $300{ }^{\circ} \mathrm{C}$ can reduce the hysteresis of the currentvoltage characteristics under illumination. Plasma treatment of $\mathrm{SnO}_{2}$ has significant positive impact on the photovoltaic device performance. Ozone-treated $\mathrm{SnO}_{x}$ was found to be the best hole blocking layer and an ITO-free semitransparent bottom electrode based on the $\mathrm{SnO}_{x} / \mathrm{Ag} / \mathrm{SnO}_{x}$ architecture has been designed. The low temperature and atmospheric pressure plasma process can be useful for the large-scale roll-to-roll functionalization of $\mathrm{SnO}_{2}$ for different applications. An emerging application of doped $\mathrm{SnO}_{2}$ is in the field of electrical energy storage devices. $\mathrm{SnO}_{2}$ has been used as a component of the composite material for the anode of LIBs and as an adsorption layer for the cathode of $\mathrm{Li}-\mathrm{S}$ batteries.

The increasing demand for optoelectronics, photovoltaics and energy storage devices requires the development of costeffective but at the same time high-performance TCOs and ETLs in terms of conductivity and transparency. The low cost, non-toxic nature, high specific capacity, high transparency in the visible range and high electron mobility properties have made tin oxide a potential material for these applications. Understanding and controlling their structure, chemical properties, surface functionalization, surface roughness and film thickness are the key to optimize the performance of the $\mathrm{SnO}_{2}$ based structures. Great contributions have been made to date, like doping $\mathrm{SnO}_{2}$ with suitable materials through several synthesis processes, functionalization of $\mathrm{SnO}_{2}$ through plasma treatment and energy band alignment through band engineering.

However, looking toward the future, there are still some challenges to overcome for the use of $\mathrm{SnO}_{2}$ in industrial applications. The use of a dopant to increase the electrical conductivity needs further screening and investigation to find the most advantageous dopant and deposition technique. A two-step sputtering method can be used to improve the crystal quality of the $\mathrm{SnO}_{2}$ without causing interface damage at the oxide/ substrate interface. Thermal stability is still a critical issue for the $\mathrm{SnO}_{2}$ based devices. By designing the suitable passivation on the substrates, it is possible to avoid the interface damage and interdiffusion of elements during thermal treatment. Therefore, oxide/ $\mathrm{SnO}_{2}$ bilayer design will play a key role in suppressing the bulk defects and recombination centers. More work is needed to achieve a precise control of composition, film quality, defects, crystallinity, and dopant activation, which will improve the conversion efficiency and stability of the device. It is important to simplify the fabrication process and use techniques like solution and plasma-based technologies for large scale and roll-to-roll deposition and sustainable production. Doped $\mathrm{SnO}_{2}$ has a huge potential for energy saving, and transparent smart window applications. However, there is a lack of investigation about the $\mathrm{SnO}_{2}$ based heat reflecting coating. Furthermore, $\mathrm{SnO}_{2}$ based cool paint can be a game changer for the super-low-energy buildings. A focused investigation on solution based doped $\mathrm{SnO}_{2}$ is required to achieve the $\mathrm{SnO}_{2}$ based cool paint to reduce the energy consumption for indoor thermal comfort.

Though a lot of work has been done, there is still a long way to go for commercializing $\mathrm{SnO}_{2}$ in the field of energy storage devices. As discussed in Section 8.1, $\mathrm{SnO}_{2}$ can be a crucial component in modifying the cathode, anode and separator in traditional LIBs. However, the specific charge capacity, specific power and cyclability all need to be improved and should be the focus of research in the near future. The synthesis of novel nanostructured $\mathrm{SnO}_{2}$ composite materials with high porosity and high specific area for supercapacitor electrodes is another important topic worthy of further investigation. The main problem associated with $\mathrm{Li}-\mathrm{S}$ batteries (which is considered as the next big thing in the energy storage field) is polysulfide shuttling and growth of Li dendrites. From our discussion, we have seen that these problems associated with $\mathrm{Li}-\mathrm{S}$ batteries can be addressed by utilizing $\mathrm{SnO}_{2}$ nanoparticles. For supercapacitors, the contemporary metal oxides like $\mathrm{NiO}, \mathrm{MnO}_{2}$, and $\mathrm{Co}_{3} \mathrm{O}_{4}$ are more favourable as supercapacitor electrodes as compared to $\mathrm{SnO}_{2}$. However, these oxides also have their disadvantages, and if the electrochemical properties of $\mathrm{SnO}_{2}$ can be tuned by controlling the morphology, or by a doping strategy or by coupling with MXenes, CNTs and conducting polymers, there is a possibility of a commercial supercapacitor prototype using the $\mathrm{SnO}_{2}$ electrode material.

The current trend of using tin oxide materials for optoelectronic and energy storage devices is a challenge that involves materials scientists and mechanical, electrical and chemical engineers. It should be recognized that doped $\mathrm{SnO}_{2}$ and doped ZnO (e.g. GZO) are complementary TCOs. In terms of materials properties, sustainability and cost, these two oxide categories are comparable and offer the materials community two good alternatives. Nevertheless, this comprehensive review article will provide key guidelines for the materials synthesis and the mapping of $\mathrm{SnO}_{2}$ based TCOs, ETLs, and electrodes for 
performance-improving strategies towards accentuating the device performance.

\section{Author contributions}

GKD/PS/SK/MS/AKC: concept/design and drafting; HS and PB: investigation (electrical properties, original draft); AG and SM: formal analysis and original draft (perovskite and transport layer); NC and AKC: formal analysis, energy storage devices (LSB, supercapacitors, electrochromics), abstract and introduction; QL, GS, and PS: investigation, formal analysis, original draft and supervision (organic and ETL, LIBS); AK, SC, and SB: synthesis, thermal treatment, and electronic properties; $\mathrm{AD}$, CSR and SK: funding acquisition, formal analysis, plasma treatment, thermal treatment and future prospect; TW: abstract, perovskite, and transparent PV, summary and outlook; SZ and SG: physical vapor deposition; CM: original draft (morphology study); Avishek Kumar: electronic properties and solution method; and SR: original draft (physical and solution methods). All authors equally commented on and edited the manuscript.

\section{Conflicts of interest}

There are no conflicts to declare.

\section{Acknowledgements}

GKD is thankful to the National University of Singapore, Global Challenges Research Fund, The Open University UK, and MingChi University of Technology-Taiwan for providing funding support to visiting faculty. Sajal Biring acknowledges the financial assistance from the Ministry of Science and Technology, Taiwan (MOST 109-2221-E-131-002). P. S. is thankful to QUT for financial support, the Australian Research Council (ARC) for the Future Fellowship (FT130101337), QUT core funding (QUT/322120-0301/07) and Centre for Materials Science, QUT for their generous support. NC and AKC acknowledge the MHRD, Govt. of India for funding under TEQIP-III (CoE), May 2018. SK acknowledges Recycling Lithium ion batteries for a sustainable technological and economic development (ReListed) DSTUKIERI-2018-19-008, Royal Society IES $\backslash$ R2 $\backslash 170272$ Royal Academy of Engineering Newton Fund and European Commission Smart innovative system for recycling wastewater project id: 958491 and creating closed loops in textile manufacturing industrial processes, and Global Challenges Research Fund on cleaning systems for solar cells.

\section{References}

1 R. A. Maniyara, V. K. Mkhitaryan, T. L. Chen, D. S. Ghosh and V. Pruneri, Nat. Commun., 2016, 7, 13771.

2 G. K. Dalapati, A. K. Kushwaha, M. Sharma, V. Suresh, S. Shannigrahi, S. Zhuk and S. Masudy-Panah, Prog. Mater. Sci., 2018, 95, 42-131.
3 S. D. Ponja, B. A. D. Williamson, S. Sathasivam, D. O. Scanlon, I. P. Parkin and C. J. Carmalt, J. Mater. Chem. C, 2018, 6, 7257-7266.

4 L. Mai, D. Zanders, E. Subaşı, E. Ciftyurek, C. Hoppe, D. Rogalla, W. Gilbert, T. d. 1. Arcos, K. Schierbaum, G. Grundmeier, C. Bock and A. Devi, ACS Appl. Mater. Interfaces, 2019, 11, 3169-3180.

5 M. J. Powell, B. A. D. Williamson, S.-Y. Baek, J. Manzi, D. B. Potter, D. O. Scanlon and C. J. Carmalt, Chem. Sci., 2018, 9, 7968-7980.

6 D. Mohanta and M. Ahmaruzzaman, RSC Adv., 2016, 6, 110996-111015.

7 Y. Zhang, C. Ma, X. Lu and M. Liu, Mater. Horiz., 2019, 6, 911-930.

8 V. Ganesh, M. Arif, M. A. Manthrammel, M. Shkir, A. Singh and S. AlFaify, Opt. Mater., 2019, 94, 277-285.

9 K. Ellmer, Nat. Photonics, 2012, 6, 809-817.

10 C.-Y. Kim and D.-H. Riu, Thin Solid Films, 2011, 519, 30813085.

11 S. Yu, L. Li, X. Lyu and W. Zhang, Sci. Rep., 2016, 6, 20399. 12 S. C. Dixon, D. O. Scanlon, C. J. Carmalt and I. P. Parkin, J. Mater. Chem. C, 2016, 4, 6946-6961.

13 J. Barbé, M. L. Tietze, M. Neophytou, B. Murali, E. Alarousu, A. E. Labban, M. Abulikemu, W. Yue, O. F. Mohammed, I. McCulloch, A. Amassian and S. Del Gobbo, ACS Appl. Mater. Interfaces, 2017, 9, 11828-11836.

14 D. Slocombe, A. Porch, M. Pepper and P. P. Edwards, Energy Environ. Sci., 2012, 5, 5387-5391.

15 R. R. Kasar, N. Deshpande, Y. Gudage, J. Vyas and R. Sharma, Phys. B, 2008, 403, 3724-3729.

16 M. J. Mortelliti, A. N. Wang and J. L. Dempsey, Polyhedron, 2019, 171, 433-447.

17 C. G. Granqvist, Sol. Energy Mater. Sol. Cells, 2007, 91, 15291598.

18 J. Sawahata and T. Kawasaki, Thin Solid Films, 2019, 685, 210-215.

19 H. B. Lee, W.-Y. Jin, M. M. Ovhal, N. Kumar and J.-W. Kang, J. Mater. Chem. C, 2019, 7, 1087-1110.

20 K. Sakamoto, H. Kuwae, N. Kobayashi, A. Nobori, S. Shoji and J. Mizuno, Sci. Rep., 2018, 8, 2825.

21 S. Ke, C. Chen, N. Fu, H. Zhou, M. Ye, P. Lin, W. Yuan, $\mathrm{X}$. Zeng, L. Chen and H. Huang, ACS Appl. Mater. Interfaces, 2016, 8, 28406-28411.

22 J. E. N. Swallow, B. A. D. Williamson, T. J. Whittles, M. Birkett, T. J. Featherstone, N. Peng, A. Abbott, M. Farnworth, K. J. Cheetham, P. Warren, D. O. Scanlon, V. R. Dhanak and T. D. Veal, Adv. Funct. Mater., 2018, 28, 1701900.

23 C. Guillén and J. Herrero, Thin Solid Films, 2011, 520, 1-17. 24 B.-j. Li, L.-j. Huang, M. Zhou, N.-f. Ren and B. Wu, Ceram. Int., 2014, 40, 1627-1633.

25 N. Noor, C. K. T. Chew, D. S. Bhachu, M. R. Waugh, C. J. Carmalt and I. P. Parkin, J. Mater. Chem. C, 2015, 3, 9359-9368.

26 Y. Zhang, H. Zhang, J. Zhang, J. Wang and Z. Li, RSC Adv., 2015, 5, 106258-106264. 
27 C.-Y. Tsay and S.-C. Liang, J. Alloys Compd., 2015, 622, 644650.

28 D. Langley, G. Giusti, C. Mayousse, C. Celle, D. Bellet and J.-P. Simonato, Nanotechnology, 2013, 24, 452001.

29 W. Zhou, R. Liu, Q. Wan, Q. Zhang, A. L. Pan, L. Guo and B. Zou, J. Phys. Chem. C, 2009, 113, 1719-1726.

30 R. Yang, Y. Gu, Y. Li, J. Zheng and X. Li, Acta Mater., 2010, 58, 866-874.

31 M. Kam, Q. Zhang, D. Zhang and Z. Fan, Sci. Rep., 2019, 9, 6963.

32 Y. Cong, D. Han, J. Dong, S. Zhang, X. Zhang and Y. Wang, Sci. Rep., 2017, 7, 1497.

33 N. Chakrabarty, M. Char, S. Krishnamurthy and A. K. Chakraborty, Mater. Adv., 2021, 2, 366-375.

34 P. Chatterjee and A. K. Chakraborty, Opt. Mater., 2021, 111, 110610.

35 S. M. D. Watson, K. S. Coleman and A. K. Chakraborty, ACS Nano, 2008, 2, 643-650.

36 N. Chakrabarty, A. Dey, S. Krishnamurthy and A. K. Chakraborty, Appl. Surf. Sci., 2021, 536, 147960.

37 P. Tiwary, R. Mahapatra and A. K. Chakraborty, J. Mater. Sci.: Mater. Electron., 2019, 30, 5464-5469.

38 S. Gupta Chatterjee, S. Dey, D. Samanta, S. Santra, S. Chatterjee, P. K. Guha and A. K. Chakraborty, J. Mater. Sci.: Mater. Electron., 2018, 29, 20162-20171.

39 S.-W. Lee, K.-J. Choi, B.-H. Kang, J.-S. Lee, S.-W. Kim, J.-B. Kwon, S.-A. Gopalan, J.-H. Bae, E.-S. Kim, D.-H. Kwon and S.-W. Kang, Org. Electron., 2016, 39, 250-257.

40 K.-S. Cho and H.-K. Kim, RSC Adv., 2018, 8, 2599-2609.

41 C. Hudaya, B. J. Jeon and J. K. Lee, ACS Appl. Mater. Interfaces, 2015, 7, 57-61.

42 J. J. Lee, J.-Y. Ha, W.-K. Choi, Y. S. Cho and J.-W. Choi, ACS Comb. Sci., 2015, 17, 247-252.

43 A. Stadler, Materials, 2012, 5, 661-683.

44 T. Minami, Thin Solid Films, 2008, 516, 1314-1321.

45 Z. Ghorannevis, E. Akbarnejad and M. Ghoranneviss, J. Theor. Appl. Phys., 2015, 9, 285-290.

46 Q. Wan, E. N. Dattoli, W. Y. Fung, W. Guo, Y. Chen, X. Pan and W. Lu, Nano Lett., 2006, 6, 2909-2915.

47 L. Passoni, F. Fumagalli, A. Perego, S. Bellani, P. Mazzolini and F. Di Fonzo, Nanotechnology, 2017, 28, 245603.

48 B. Yang, C. Yao, Y. Yu, Z. Li and X. Wang, Sci. Rep., 2017, 7, 4936.

49 K. Higashitani, C. E. McNamee and M. Nakayama, Langmuir, 2011, 27, 2080-2083.

50 S. Das and V. Jayaraman, Prog. Surf. Sci., 2014, 66, 112-255.

51 K. Jenifer, S. Arulkumar, S. Parthiban and J. Y. Kwon, J. Electron. Mater., 2020, 49, 7098-7111.

52 A. M. Al-Hamdi, U. Rinner and M. Sillanpää, Process Saf. Environ. Prot., 2017, 107, 190-205.

53 Q. Jiang, X. Zhang and J. You, Small, 2018, 14, 1801154.

54 A. M. Ganose and D. O. Scanlon, J. Mater. Chem. C, 2016, 4, 1467-1475.

55 M. Park, K. Jae-Yup, H. Son, C.-H. Lee, S. Jang and M. Ko, Nano Energy, 2016, 26, 208-215.

56 Y. Bai, Y. Fang, Y. Deng, Q. Wang, J. Zhao, X. Zheng, Y. Zhang and J. Huang, ChemSusChem, 2016, 9, 2686-2691.
57 L. Xiong, Y. Guo, J. Wen, H. Liu, G. Yang, P. Qin and G. Fang, Adv. Funct. Mater., 2018, 28, 1802757.

58 Y. Chen, Q. Meng, L. Zhang, C. Han, H. Gao, Y. Zhang and H. Yan, J. Energy Chem., 2019, 35, 144-167.

59 S. H. Saeedabad, G. S. Selopal, S. M. Rozati, Y. Tavakoli and G. Sberveglieri, J. Electron. Mater., 2018, 47, 5165-5173.

60 R. Milan, G. S. Selopal, M. Epifani, M. M. Natile, G. Sberveglieri, A. Vomiero and I. Concina, Sci. Rep., 2015, $5,14523$.

61 R. Milan, G. Singh Selopal, M. Cavazzini, S. Orlandi, R. Boaretto, S. Caramori, I. Concina and G. Pozzi, Sci. Rep., 2020, 10, 1176.

62 K. Basu, G. S. Selopal, M. Mohammadnezad, R. Akilimali, Z. M. Wang, H. Zhao, F. Vetrone and F. Rosei, Electrochim. Acta, 2020, 349, 136409.

63 Q. Dong, J. Li, Y. Shi, M. Chen, L. K. Ono, K. Zhou, C. Zhang, Y. Qi, Y. Zhou, N. P. Padture and L. Wang, Adv. Energy Mater., 2019, 9, 1900834.

64 P. Shen, M. Yao, G. Wang, R. Mi, W. Guo, Y. Bai and L. Shen, J. Mater. Chem. A, 2018, 6, 17401-17408.

65 D. Yang, R. Yang, K. Wang, C. Wu, X. Zhu, J. Feng, X. Ren, G. Fang, S. Priya and S. Liu, Nat. Commun., 2018, 9, 3239.

66 S. Castro-Hermosa, G. Lucarelli, M. Top, M. Fahland, J. Fahlteich and T. M. Brown, Cell Rep. Phys. Sci., 2020, 1, 100045.

67 M. Park, J. Roh, J. Lim, H. Lee and D. Lee, Nanomaterials, 2020, 10, 726.

68 Y. Liu, S. Wei, G. Wang, J. Tong, J. Li and D. Pan, Langmuir, 2020, 36, 6605-6609.

69 Y. Deng, C. Fang and G. Chen, J. Power Sources, 2016, 304, 81-101.

70 K. C. Mishra, K. H. Johnson and P. C. Schmidt, Phys. Rev. B: Condens. Matter Mater. Phys., 1995, 51, 13972-13976.

71 M. Batzill and U. Diebold, Prog. Surf. Sci., 2005, 79, 47-154.

72 Ç. Kılıç and A. Zunger, Phys. Rev. Lett., 2002, 88, 095501.

73 K. B. Sundaram and G. K. Bhagavat, J. Phys. D: Appl. Phys., 1981, 14, 921-925.

74 C. Ke, W. Zhu, Z. Zhang, E. Soon Tok, B. Ling and J. Pan, Sci. Rep., 2015, 5, 17424.

75 Y. W. Noh, J. H. Lee, I. S. Jin, S. H. Park and J. W. Jung, Nano Energy, 2019, 65, 104014.

76 L. Xiong, M. Qin, G. Yang, Y. Guo, H. Lei, Q. Liu, W. Ke, H. Tao, P. Qin, S. Li, H. Yu and G. Fang, J. Mater. Chem. A, 2016, 4, 8374-8383.

77 D. Wang, S.-C. Chen and Q. Zheng, J. Mater. Chem. C, 2019, 7, 12204-12210.

78 J. Song, W. Zhang, D. Wang, K. Deng, J. Wu and Z. Lan, Sol. Energy, 2019, 185, 508-515.

79 H. Ye, Z. Liu, X. Liu, B. Sun, X. Tan, Y. Tu, T. Shi, Z. Tang and G. Liao, Appl. Surf. Sci., 2019, 478, 417-425.

80 Z. Xu, S. H. Teo, L. Gao, Z. Guo, Y. Kamata, S. Hayase and T. Ma, Org. Electron., 2019, 73, 62-68.

81 Z. Ma, W. Zhou, Z. Xiao, H. Zhang, Z. Li, J. Zhuang, C. Peng and Y. Huang, Org. Electron., 2019, 71, 98-105.

82 R. Ramarajan, N. Purushothamreddy, R. K. Dileep, M. Kovendhan, G. Veerappan, K. Thangaraju and D. Paul Joseph, Sol. Energy, 2020, 211, 547-559. 
83 O. S. Elsherif, G. E. A. Muftah, O. Abubaker and I. M. Dharmadasa, J. Mater. Sci.: Mater. Electron., 2016, 27, 12280-12286.

84 A. Way, J. Luke, A. D. Evans, Z. Li, J.-S. Kim, J. R. Durrant, H. K. H. Lee and W. C. Tsoi, AIP Adv., 2019, 9, 085220.

85 S. Das and V. Jayaraman, Prog. Mater. Sci., 2014, 66, 112255.

86 B. Bissig, T. Jäger, L. Ding, A. N. Tiwari and Y. E. Romanyuk, APL Mater., 2015, 3, 062802.

87 E. Elangovan and K. Ramamurthi, Thin Solid Films, 2005, 476, 231-236.

88 S. Gürakar, T. Serin and N. Serin, Appl. Surf. Sci., 2015, 352, 16-22.

89 Z. Y. K. Banyamin, P. J. Kelly, G. West and J. Boardman, Coatings, 2014, 4, 732-746.

90 Ç. Kılıç and A. Zunger, Phys. Rev. Lett., 2002, 88, 095501.

91 V. Sharma, R. Vyas, P. Bazylewski, G. S. Chang, K. Asokan and K. Sachdev, RSC Adv., 2016, 6, 29135-29141.

92 J. Heo, A. S. Hock and R. G. Gordon, Chem. Mater., 2010, 22, 4964-4973.

93 M. A. Hossain, J. R. Jennings, Z. Y. Koh and Q. Wang, ACS Nano, 2011, 5, 3172-3181.

94 J. Liu, T. Luo, T. S. Mouli, F. Meng, B. Sun, M. Li and J. Liu, Chem. Commun., 2010, 46, 472-474.

95 F. Gyger, M. Hübner, C. Feldmann, N. Barsan and U. Weimar, Chem. Mater., 2010, 22, 4821-4827.

96 S. Kang, R. Nandi, J.-K. Sim, J.-Y. Jo, U. Chatterjee and C.-R. Lee, $R S C A d v .$, 2017, 7, 48113-48119.

97 M. Esro, S. Georgakopoulos, H. Lu, G. Vourlias, A. Krier, W. I. Milne, W. P. Gillin and G. Adamopoulos, J. Mater. Chem. C, 2016, 4, 3563-3570.

98 X. Yu, T. J. Marks and A. Facchetti, Nat. Mater., 2016, 15, 383-396.

99 X. Huang, J. Du, X. Guo, Z. Lin, J. Ma, J. Su, L. Feng, C. Zhang, J. Zhang, J. Chang and Y. Hao, Sol. RRL, 2020, 4, 1900336.

100 D. Cheng, M. Zhang, J. Chen, C. Yang, X. Zeng and D. Cao, J. Phys. Chem. C, 2014, 118, 2037-2043.

101 S. Yu, W. Zhang, L. Li, D. Xu, H. Dong and Y. Jin, Appl. Surf. Sci., 2013, 286, 417-420.

102 W. Yang, S. Yu, Y. Zhang and W. Zhang, Thin Solid Films, 2013, 542, 285-288.

103 B.-j. Li, L.-j. Huang, N.-f. Ren, X. Kong, Y.-l. Cai and J.-l. Zhang, Appl. Surf. Sci., 2015, 351, 113-118.

104 A. H. Omran Alkhayatt and S. K. Hussian, Mater. Lett., 2015, 155, 109-113.

105 K. Anandan and V. Rajendran, Superlattices Microstruct., 2015, 85, 185-197.

106 G. Turgut, Thin Solid Films, 2015, 594, 56-66.

107 S. Nakao, N. Yamada, T. Hitosugi, Y. Hirose, T. Shimada and T. Hasegawa, Thin Solid Films, 2010, 518, 3093-3096.

108 S.-H. Cho, R. Pandey, C.-H. Wie, Y. J. Lee, J.-W. Lim, D.-H. Park, J.-S. Seok, Y.-H. Jang, K.-K. Kim, D. K. Hwang, D.-J. Byun and W.-K. Choi, Phys. Status Solidi B, 2014, 211, 1860-1867.

109 L. Soussi, T. Garmim, O. Karzazi, A. Rmili, A. El Bachiri, A. Louardi and H. Erguig, Surf. Interfaces, 2020, 19, 100467.
110 S. Sambasivam, P. S. Maram, C. V. V. Muralee Gopi and I. M. Obaidat, Optik, 2020, 202, 163596.

111 L. Zhang, W. Xu, W. Liu, P. Cao, S. Han, D. Zhu and Y. Lu, J. Phys. D: Appl. Phys., 2020, 53, 175106.

112 Q. Chen and E. Thimsen, ACS Appl. Mater. Interfaces, 2020, 12, 25168-25177.

113 J. Ni, X. Zhao, X. Zheng, J. Zhao and B. Liu, Acta Mater., 2009, 57, 278-285.

114 J. Ni, X. Zhao and J. Zhao, J. Inorg. Organomet. Polym. Mater., 2012, 22, 21-26.

115 F. Fang, Y. Zhang, X. Wu, Q. Shao and Z. Xie, Mater. Res. Bull., 2015, 68, 240-244.

116 H. Liu, V. Avrutin, N. Izyumskaya, Ü. Özgür and H. Morkoç, Superlattices Microstruct., 2010, 48, 458-484.

117 G. Turgut, E. F. Keskenler, S. Aydın, E. Sönmez, S. Doğan, B. Düzgün and M. Ertuğrul, Superlattices Microstruct., 2013, 56, 107-116.

118 L. Wang, J. Yu, X. Niu, L. Wang, C. Fu, R. Qiu, W. Yan, H. Zhao and J. Yang, Thin Solid Films, 2018, 649, 147-153.

119 X. Huo, S. Jiang, P. Liu, M. Shen, S. Qiu and M.-Y. Li, CrystEngComm, 2017, 19, 4413-4423.

120 Y. J. Wu, Y. S. Liu, C. Y. Hsieh, P. M. Lee, Y. S. Wei, C. H. Liao and C. Y. Liu, Appl. Surf. Sci., 2015, 328, 262-268.

121 T. Entradas, J. F. Cabrita, S. Dalui, M. R. Nunes, O. C. Monteiro and A. J. Silvestre, Mater. Chem. Phys., 2014, 147, 563-571.

122 C. E. Benouis, M. Benhaliliba, Z. Mouffak, A. Avila-Garcia, A. Tiburcio-Silver, M. Ortega Lopez, R. Romano Trujillo and Y. S. Ocak, J. Alloys Compd., 2014, 603, 213-223.

123 S. K. Sinha, S. K. Ray and I. Manna, Philos. Mag., 2014, 94, 3507-3521.

124 J. Zhao, X. J. Zhao, J. M. Ni and H. Z. Tao, Acta Mater., 2010, 58, 6243-6248.

125 S. Wang, L. Shi, G. Chen, C. Ba, Z. Wang, J. Zhu, Y. Zhao, M. Zhang and S. Yuan, ACS Appl. Mater. Interfaces, 2017, 9, 17163-17171.

126 G. Zhang, X. Zhang, H. Ning, H. Chen, Q. Wu, M. Jiang, C. Li, D. Guo, Y. Wang, R. Yao and J. Peng, Superlattices Microstruct., 2019, 130, 277-284.

127 M. S. Bannur, A. Antony, K. I. Maddani, A. Ani, P. Poornesh, A. Rao, K. S. Choudhari and S. D. Kulkarni, Opt. Mater., 2019, 94, 294-298.

128 L. He, C. Luan, X. Feng, H. Xiao, X. Yang, D. Wang and J. Ma, Ceram. Int., 2019, 45, 10196-10202.

129 F. Lungwitz, R. Escobar-Galindo, D. Janke, E. Schumann, R. Wenisch, S. Gemming and M. Krause, Sol. Energy Mater. Sol. Cells, 2019, 196, 84-93.

130 Z. Zhang, C. Yin, L. Yang, J. Jiang and Y. Guo, J. Alloys Compd., 2019, 785, 819-825.

131 G. Brunin, F. Ricci, V.-A. Ha, G.-M. Rignanese and G. Hautier, npj Comput. Mater., 2019, 5, 63.

132 H. Peng, A. Zakutayev, S. Lany, T. R. Paudel, M. d'Avezac, P. F. Ndione, J. D. Perkins, D. S. Ginley, A. R. Nagaraja, N. H. Perry, T. O. Mason and A. Zunger, Adv. Funct. Mater., 2013, 23, 5267-5276.

133 G. Hautier, A. Miglio, G. Ceder, G.-M. Rignanese and X. Gonze, Nat. Commun., 2013, 4, 2292. 
134 F. Borgatti, J. A. Berger, D. Céolin, J. S. Zhou, J. J. Kas, M. Guzzo, C. F. McConville, F. Offi, G. Panaccione, A. Regoutz, D. J. Payne, J.-P. Rueff, O. Bierwagen, M. E. White, J. S. Speck, M. Gatti and R. G. Egdell, Phys. Rev. B, 2018, 97, 155102.

135 M. Kim, N. Marom, N. S. Bobbitt and J. R. Chelikowsky, J. Chem. Phys., 2015, 142, 044704.

136 Z. Chen, Q. Xie, J. Ding, Z. Yang, W. Zhang and H. Cheng, ACS Appl. Mater. Interfaces, 2020, 12, 29937-29945.

137 B. A. D. Williamson, T. J. Featherstone, S. S. Sathasivam, J. E. N. Swallow, H. Shiel, L. A. H. Jones, M. J. Smiles, A. Regoutz, T.-L. Lee, X. Xia, C. Blackman, P. K. Thakur, C. J. Carmalt, I. P. Parkin, T. D. Veal and D. O. Scanlon, Chem. Mater., 2020, 32, 1964-1973.

138 F. E. Ghodsi and J. Mazloom, Appl. Phys. A: Mater. Sci. Process., 2012, 108, 693-700.

139 R. M. Pasquarelli, D. S. Ginley and R. O'Hayre, Chem. Soc. Rev., 2011, 40, 5406-5441.

140 H.-R. An, C. Kim, S.-T. Oh and H.-J. Ahn, Ceram. Int., 2014, 40, 385-391.

141 K. Bouras, G. Schmerber, D. Aureau, H. Rinnert, J.-L. Rehspringer, D. Ihiawakrim, A. Dinia, A. Slaoui and S. Colis, Phys. Chem. Chem. Phys., 2019, 21, 21407-21417.

142 A. E. Danks, S. R. Hall and Z. Schnepp, Mater. Horiz., 2016, 3, 91-112.

143 S. Lekshmy, G. Daniel and K. Joy, Appl. Surf. Sci., 2013, 274, 95-100.

144 X. H. Shi and K. J. Xu, Mater. Sci. Semicond. Process., 2017, $58,1-7$.

145 T. J. Liu, Z. G. Jin, L. R. Feng and T. Wang, Appl. Surf. Sci., 2008, 254, 6547-6553.

146 Y. Wang, T. Brezesinski, M. Antonietti and B. Smarsly, ACS Nano, 2009, 3, 1373-1378.

147 Y. Huang, Z. Ji and C. Chen, Appl. Surf. Sci., 2007, 253, 4819-4822.

148 H. Uchiyama, T. Ito, R. Sasaki and H. Kozuka, RSC Adv., 2015, 5, 20371-20375.

149 Q.-P. Tran, J.-S. Fang and T.-S. Chin, Mater. Sci. Semicond. Process., 2015, 40, 664-669.

150 J. Zhao, R. Tan, Y. Yang, W. Xu, J. Li, W. Shen, G. Wu, X. Yang and W. Song, J. Mater. Sci. Technol., 2015, 31, $815-821$.

151 L. Luo, D. Bozyigit, V. Wood and M. Niederberger, Chem. Mater., 2013, 25, 4901-4907.

152 A. Nadarajah, M. E. Carnes, M. G. Kast, D. W. Johnson and S. W. Boettcher, Chem. Mater., 2013, 25, 4080-4087.

153 K. Tsukuma, T. Akiyama and H. Imai, J. Non-Cryst. Solids, 1997, 210, 48-54.

154 N. Cai and J. Cho, Ceram. Int., 2013, 39, 143-151.

155 D. Raviendra and J. K. Sharma, J. Phys. Chem. Solids, 1985, 46, 945-950.

156 Y. Ren, G. Zhao and Y. Chen, Appl. Surf. Sci., 2011, 258, 914918.

157 G. R. A. Kumara, C. S. K. Ranasinghe, E. N. Jayaweera, H. M. N. Bandara, M. Okuya and R. M. G. Rajapakse, J. Phys. Chem. C, 2014, 118, 16479-16485.
158 D. Miao, Q. Zhao, S. Wu, Z. Wang, X. Zhang and X. Zhao, J. Non-Cryst. Solids, 2010, 356, 2557-2561.

159 A. V. Moholkar, S. M. Pawar, K. Y. Rajpure and C. H. Bhosale, J. Alloys Compd., 2008, 455, 440-446.

160 S. P. Choudhury, S. D. Gunjal, N. Kumari, K. D. Diwate, K. C. Mohite and A. Bhattacharjee, Mater. Today: Proc., 2016, 3, 1609-1619.

161 B. Benhaoua, S. Abbas, A. Rahal, A. Benhaoua and M. S. Aida, Superlattices Microstruct., 2015, 83, 78-88.

162 A. Muthukumar, G. Giusti, M. Jouvert, V. Consonni and D. Bellet, Thin Solid Films, 2013, 545, 302-309.

163 G. Turgut, E. F. Keskenler, S. Aydın, M. Yılmaz, S. Doğan and B. Düzgün, Phys. Scr., 2013, 87, 035602.

164 V. Gokulakrishnan, S. Parthiban, K. Jeganathan and K. Ramamurthi, J. Mater. Sci., 2011, 46, 5553-5558.

165 K. D. Arun Kumar, S. Valanarasu, A. Kathalingam and K. Jeyadheepan, Mater. Res. Bull., 2018, 101, 264-271.

166 T. Serin, N. Serin, S. Karadeniz, H. Sari, N. Tuğluoğlu and O. Pakma, J. Non-Cryst. Solids, 2006, 352, 209-215.

167 M.-M. Bagheri-Mohagheghi and M. Shokooh-Saremi, Phys. $B, 2010,405,4205-4210$.

168 J. T. Wang, X. L. Shi, W. W. Liu, X. H. Zhong, J. N. Wang, L. Pyrah, K. D. Sanderson, P. M. Ramsey, M. Hirata and K. Tsuri, Sci. Rep., 2014, 4, 3679.

169 L. He, C. Luan, X. Feng, H. Xiao, X. Yang, D. Wang and J. Ma, Mater. Res. Bull., 2019, 118, 110488.

170 M.-Y. Tsai, O. Bierwagen and J. S. Speck, Thin Solid Films, 2016, 605, 186-192.

171 S. Yu, L. Zhao, R. Liu, M. Wu, Y. Sun and L. Li, Ceram. Int., 2019, 45, 2201-2206.

172 H. S. So, J.-W. Park, D. H. Jung, K. H. Ko and H. Lee, J. Appl. Phys., 2015, 118, 085303.

173 J. Montero, C. Guillén, C. Granqvist, J. Herrero and G. Niklasson, ECS J. Solid State Sci. Technol., 2014, 3, N151-N153.

174 P.-C. Hsu, C.-J. Hsu, C.-H. Chang, S.-P. Tsai, W.-C. Chen, H.-H. Hsieh and C.-C. Wu, ACS Appl. Mater. Interfaces, 2014, 6, 13724-13729.

175 K. Y. Park, H. J. Cho, T. K. Song, H. J. Ko and B. H. Koo, Trans. Nonferrous Met. Soc. China, 2014, 24, s129-s133.

176 G. J. Exarhos and X.-D. Zhou, Thin Solid Films, 2007, 515, 7025-7052.

177 M. Weidner, J. Brötz and A. Klein, Thin Solid Films, 2014, 555, 173-178.

178 X. Ding, F. Fang and J. Jiang, Surf. Coat. Technol., 2013, 231, 67-70.

179 J. Boltz, D. Koehl and M. Wuttig, Surf. Coat. Technol., 2010, 205, 2455-2460.

180 T. Minami and T. Miyata, Thin Solid Films, 2008, 517, 14741477.

181 T. Minami, Thin Solid Films, 2008, 516, 5822-5828.

182 J. Gong, X. Wang, X. Fan, R. Dai, Z. Wang, Z. Zhang and Z. Ding, Opt. Mater. Express, 2019, 9, 3691-3699.

183 S. Yu, L. Li, Z. Sun, H. Zheng, H. Dong, D. Xu and W. Zhang, J. Am. Ceram. Soc., 2015, 98, 1121-1127.

184 B. Parida, Y. Gil and H. Kim, J. Nanosci. Nanotechnol., 2019, 19, 1455-1462. 
185 B.-H. Liao, S.-H. Chan, C.-C. Lee, C.-C. Kuo, S.-H. Chen and D. Chiang, Appl. Opt., 2014, 53, A148-A153.

186 H.-R. Kim, G.-H. Lee and D.-H. Kim, J. Phys. D: Appl. Phys., 2011, 44, 185203.

187 B. L. Zhu, F. Liu, K. Li, K. Lv, J. Wu, Z. H. Gan, J. Liu, D. W. Zeng and C. S. Xie, Ceram. Int., 2017, 43, 1028810298.

188 F. I. Chowdhury, T. Blaine and A. B. Gougam, Energy Procedia, 2013, 42, 660-669.

189 T. Ishihara, K. Matsuzawa, M. Takayanagi and S.-i. Takagi, Jpn. J. Appl. Phys., 2002, 41, 2353-2358.

190 D. Kim, A. W. H. Lee, J. I. Eastcott and B. D. Gates, ACS Appl. Nano Mater., 2018, 1, 2237-2248.

191 D.-J. Yun, D.-K. Lee, H.-K. Jeon and S.-W. Rhee, Org. Electron., 2007, 8, 690-694.

192 Ľ. Scholtz, L. Ladányi and J. Mullerova, Adv. Electr. Electron. Eng., 2015, 12, 631-638.

193 B. Cao, X. He, J. B. Sorge, A. Lalany, K. Ahadi, A. Afshar, B. C. Olsen, T. C. Hauger, M. H. Mobarok, P. Li, K. C. Cadien, M. J. Brett, E. J. Luber and J. M. Buriak, ACS Appl. Mater. Interfaces, 2017, 9, 38706-38715.

194 V. Consonni, G. Rey, H. Roussel and D. Bellet, J. Appl. Phys., 2012, 111, 033523.

195 J. T. Wang, X. L. Shi, X. H. Zhong, J. N. Wang, L. Pyrah, K. D. Sanderson, P. M. Ramsey, M. Hirata and K. Tsuri, Sol. Energy Mater. Sol. Cells, 2015, 132, 578-588.

196 G. W. Kim, C. H. Sung, M. S. Anwar, Y. J. Seo, S. N. Heo, K. Y. Park, T. K. Song and B. H. Koo, Curr. Appl. Phys., 2012, 12, S21-S24.

197 M. Fukumoto, S. Nakao, K. Shigematsu, D. Ogawa, K. Morikawa, Y. Hirose and T. Hasegawa, Sci. Rep., 2020, 10, 6844 .

198 T. T. Nguyen, H. P. Dang, Q. H. Luc and T. Le, Ceram. Int., 2019, 45, 9147-9156.

199 J. Montero, J. Herrero and C. Guillén, Sol. Energy Mater. Sol. Cells, 2010, 94, 612-616.

200 S. Yu, L. Li, D. Xu, D. Helei and Y. Jin, Thin Solid Films, 2014, 562, 501-505.

201 J. Ni, X. Zhao and J. Zhao, Surf. Coat. Technol., 2012, 206, 4356-4361.

202 M. Weidner, J. Jia, Y. Shigesato and A. Klein, Phys. Status Solidi B, 2016, 253, 923-928.

203 F. de Moure-Flores, J. G. Quiñones-Galván, A. HernándezHernández, A. Guillén-Cervantes, M. A. Santana-Aranda, M. d. 1. L. Olvera and M. Meléndez-Lira, Appl. Surf. Sci., 2012, 258, 2459-2463.

204 V. Consonni, G. Rey, H. Roussel and D. Bellet, J. Appl. Phys., 2012, 111, 033523.

205 D.-H. Kim, K.-S. Cho and H.-K. Kim, Sci. Rep., 2017, 7, 2550.

206 T.-K. Kim and G.-E. Jang, J. Nanosci. Nanotechnol., 2019, 19, 1673-1676.

207 R. Pandey, C. H. Wie, X. Lin, J. W. Lim, K. K. Kim, D. K. Hwang and W. K. Choi, Sol. Energy Mater. Sol. Cells, 2015, 134, 5-14.

208 S. W. Hwang, J. U. Kim, J. H. Baek, S. S. Kalanur, H. S. Jung, H. Seo and I. S. Cho, J. Alloys Compd., 2019, 785, 1245-1252.
209 C.-H. Lee, R. Pandey, B.-Y. Wang, W.-K. Choi, D.-K. Choi and Y.-J. Oh, Sol. Energy Mater. Sol. Cells, 2015, 132, 80-85.

210 R. Alvarez, J. C. González, J. P. Espinós, A. R. GonzálezElipe, A. Cueva and F. Villuendas, Appl. Surf. Sci., 2013, 268, 507-515.

211 M.-H. Hwang, H. Kong, J.-W. Jeong and H.-Y. Lee, Superlattices Microstruct., 2020, 141, 106503.

212 A. Bou, P. Torchio, D. Barakel, A. Guillou, B. Ayachi, P.-Y. Thoulon and M. Ricci, J. Phys. D: Appl. Phys., 2015, 48, 205102.

213 M. Park, J. Song, M. An, J. Lim, C. Lee, J. Roh and D. Lee, RSC Adv., 2020, 10, 8261-8265.

214 K. Hong, K. Kim, S. Kim, S. Y. Kim and J.-L. Lee, J. Phys. Chem. C, 2011, 115, 23107-23112.

215 H. Lee, C.-M. Kang, M. Park, J. Kwak and C. Lee, ACS Appl. Mater. Interfaces, 2013, 5, 1977-1981.

216 G. Saianand, P. Sonar, G. J. Wilson, A.-I. Gopalan, V. A. L. Roy, G. E. Unni, K. Mamun Reza, B. Bahrami, K. Venkatramanan and Q. Qiao, J. Energy Chem., 2021, 54, 151-173.

217 K. M. Reza, A. Gurung, B. Bahrami, S. Mabrouk, H. Elbohy, R. Pathak, K. Chen, A. H. Chowdhury, M. T. Rahman, S. Letourneau, H.-C. Yang, G. Saianand, J. W. Elam, S. B. Darling and Q. Qiao, J. Energy Chem., 2020, 44, 41-50.

218 S.-A. Gopalan, A.-I. Gopalan, A. Vinu, K.-P. Lee and S.-W. Kang, Sol. Energy Mater. Sol. Cells, 2018, 174, 112-123.

219 B. Xu, S.-A. Gopalan, A.-I. Gopalan, N. Muthuchamy, K.-P. Lee, J.-S. Lee, Y. Jiang, S.-W. Lee, S.-W. Kim, J.-S. Kim, H.-M. Jeong, J.-B. Kwon, J.-H. Bae and S.-W. Kang, Sci. Rep., 2017, 7, 45079.

220 G. Sai-Anand, A.-I. Gopalan, K.-P. Lee, S. Venkatesan, Q. Qiao, B.-H. Kang, S.-W. Lee, J.-S. Lee and S.-W. Kang, Sol. Energy Mater. Sol. Cells, 2016, 153, 148-163.

221 G. Sai-Anand, A.-I. Gopalan, K.-P. Lee, S. Venkatesan, B.-H. Kang, S.-W. Lee, J.-S. Lee, Q. Qiao, D.-H. Kwon and S.-W. Kang, Sol. Energy Mater. Sol. Cells, 2015, 141, 275-290.

222 B. Xu, G. Sai-Anand, H.-M. Jeong, S.-W. Kim, J.-S. Kim, J.-B. Kwon and S.-W. Kang, Materials, 2018, 11, 1143.

223 B. Xu, G. Sai-Anand, G. E. Unni, H.-M. Jeong, J.-S. Kim, S.-W. Kim, J.-B. Kwon, J.-H. Bae and S.-W. Kang, Appl. Surf. Sci., 2019, 484, 825-834.

224 G. Sai-Anand, A. Dubey, A.-I. Gopalan, S. Venkatesan, S. Ruban, K. M. Reza, J. Choi, K. S. Lakhi, B. Xu, Q. Qiao and A. Vinu, Sol. Energy Mater. Sol. Cells, 2018, 182, 246254.

225 V.-H. Tran, H. Park, S. H. Eom, S. C. Yoon and S.-H. Lee, ACS Omega, 2018, 3, 18398-18410.

226 S. Trost, A. Behrendt, T. Becker, A. Polywka, P. Görrn and T. Riedl, Adv. Energy Mater., 2015, 5, 1500277.

227 S. Trost, T. Becker, A. Polywka, P. Görrn, M. F. Oszajca, N. A. Luechinger, D. Rogalla, M. Weidner, P. Reckers, T. Mayer and T. Riedl, Adv. Energy Mater., 2016, 6, 1600347.

228 Y. Geng, T. Zhao, G. Lian, X. Cui, Y. Liu, J. Liu, Q. Wang and D. Cui, RSC Adv., 2016, 6, 2387-2393.

229 V.-H. Tran, S.-K. Kim and S.-H. Lee, ACS Omega, 2019, 4, 19225-19237. 
230 Y. Jiang, L. Sun, F. Jiang, C. Xie, L. Hu, X. Dong, F. Qin, T. Liu, L. Hu, X. Jiang and Y. Zhou, Mater. Horiz., 2019, 6, 1438-1443.

231 R. Peng, T. Yan, J. Chen, S. Yang, Z. Ge and M. Wang, Adv. Electron. Mater., 2020, 6, 1901245.

232 F. Zhao, L. Deng, K. Wang, C. Han, Z. Liu, H. Yu, J. Li and B. Hu, ACS Appl. Mater. Interfaces, 2020, 12, 5120-5127.

233 H. M. Yates, M. Afzaal, A. Walter, J. L. Hodgkinson, S.-J. Moon, D. Sacchetto, M. Bräuninger, B. Niesen, S. Nicolay, M. McCarthy, M. E. Pemble, I. M. Povey and C. Ballif, J. Mater. Chem. C, 2016, 4, 11269-11277.

234 J. P. Correa Baena, L. Steier, W. Tress, M. Saliba, S. Neutzner, T. Matsui, F. Giordano, T. J. Jacobsson, A. R. Srimath Kandada, S. M. Zakeeruddin, A. Petrozza, A. Abate, M. K. Nazeeruddin, M. Grätzel and A. Hagfeldt, Energy Environ. Sci., 2015, 8, 2928-2934.

235 M. Afzaal, H. M. Yates, A. Walter, S. Nicolay and C. Ballif, J. Mater. Chem. C, 2017, 5, 4946-4950.

236 W. Ke, G. Fang, Q. Liu, L. Xiong, P. Qin, H. Tao, J. Wang, H. Lei, B. Li, J. Wan, G. Yang and Y. Yan, J. Am. Chem. Soc., 2015, 137, 6730-6733.

237 E. H. Anaraki, A. Kermanpur, L. Steier, K. Domanski, T. Matsui, W. Tress, M. Saliba, A. Abate, M. Grätzel, A. Hagfeldt and J.-P. Correa-Baena, Energy Environ. Sci., 2016, 9, 3128-3134.

238 Z. Zhu, Y. Bai, X. Liu, C.-C. Chueh, S. Yang and A. K.-Y. Jen, Adv. Mater., 2016, 28, 6478-6484.

239 Q. Ye, Y. Zhao, S. Mu, F. Ma, F. Gao, Z. Chu, Z. Yin, P. Gao, X. Zhang and J. You, Adv. Mater., 2019, 31, 1905143.

240 P. Wang, R. Li, B. Chen, F. Hou, J. Zhang, Y. Zhao and X. Zhang, Adv. Mater., 2020, 32, 1905766.

241 M. Park, J.-Y. Kim, H. J. Son, C.-H. Lee, S. S. Jang and M. J. Ko, Nano Energy, 2016, 26, 208-215.

242 Q. Liu, X. Zhang, C. Li, H. Lu, Z. Weng, Y. Pan, W. Chen, X.-C. Hang, Z. Sun and Y. Zhan, Appl. Phys. Lett., 2019, 115, 143903.

243 K.-H. Jung, J.-Y. Seo, S. Lee, H. Shin and N.-G. Park, J. Mater. Chem. A, 2017, 5, 24790-24803.

244 Y. Luan, X. Yi, P. Mao, Y. Wei, J. Zhuang, N. Chen, T. Lin, C. Li and J. Wang, iScience, 2019, 16, 433-441.

245 I. Levine, P. K. Nayak, J. T.-W. Wang, N. Sakai, S. Van Reenen, T. M. Brenner, S. Mukhopadhyay, H. J. Snaith, G. Hodes and D. Cahen, J. Phys. Chem. C, 2016, 120, 16399-16411.

246 E. Castro, J. Murillo, O. Fernandez-Delgado and L. Echegoyen, J. Mater. Chem. C, 2018, 6, 2635-2651.

247 T. Bu, S. Shi, J. Li, Y. Liu, J. Shi, L. Chen, X. Liu, J. Qiu, Z. Ku, Y. Peng, J. Zhong, Y.-B. Cheng and F. Huang, ACS Appl. Mater. Interfaces, 2018, 10, 14922-14929.

248 M. H. Kumar, N. Yantara, S. Dharani, M. Graetzel, S. Mhaisalkar, P. P. Boix and N. Mathews, Chem. Commun., 2013, 49, 11089-11091.

249 J. Feng, X. Zhu, Z. Yang, X. Zhang, J. Niu, Z. Wang, S. Zuo, S. Priya, S. Liu and D. Yang, Adv. Mater., 2018, 30, 1801418.

250 N. Zhu, X. Qi, Y. Zhang, G. Liu, C. Wu, D. Wang, X. Guo, W. Luo, X. Li, H. Hu, Z. Chen, L. Xiao and B. Qu, ACS Appl. Energy Mater., 2019, 2, 3676-3682.
251 X. Qiu, B. Yang, H. Chen, G. Liu, Y. Liu, Y. Yuan, H. Huang, H. Xie, D. Niu, Y. Gao and C. Zhou, Org. Electron., 2018, 58, 126-132.

252 C. Liu, L. Zhang, X. Zhou, J. Gao, W. Chen, X. Wang and B. Xu, Adv. Funct. Mater., 2019, 29, 1807604.

253 T. Bu, J. Li, F. Zheng, W. Chen, X. Wen, Z. Ku, Y. Peng, J. Zhong, Y.-B. Cheng and F. Huang, Nat. Commun., 2018, 9, 4609.

254 S. Albrecht, M. Saliba, J. P. Correa Baena, F. Lang, L. Kegelmann, M. Mews, L. Steier, A. Abate, J. Rappich, L. Korte, R. Schlatmann, M. K. Nazeeruddin, A. Hagfeldt, M. Grätzel and B. Rech, Energy Environ. Sci., 2016, 9, 81-88.

255 F. Liang, Y. Lin, Z. He, W. Chen, Y. Zhu, T. Chen, L. Liang, S. Ma, Y. Wu, B. Tu, D. Wang, Z. Zhang, L. Luo and Z. He, J. Mater. Chem. A, 2018, 6, 19330-19337.

256 S. A. Hashemi, S. Ramakrishna and A. G. Aberle, Energy Environ. Sci., 2020, 13, 685-743.

257 J. Tian, J. Zhang, X. Li, B. Cheng, J. Yu and W. Ho, Sol. RRL, 2020, 4, 2000090.

258 A. A. Qureshi, S. Javed, H. M. A. Javed, A. Akram, M. S. Mustafa, U. Ali and M. Z. Nisar, Mater. Sci. Semicond. Process., 2021, 123, 105545.

259 Z. Li, W. Shen, J. Zhao, H. Ying, Z. Wu, Y. Liu, W. Li, Z. Ku, Y. Peng, F. Huang, Y. Cheng, J. Zhong and Z. Fu, J. Wuhan Univ. Technol., Mater. Sci. Ed., 2020, 35, 272-279.

260 S. Sujinnapram and S. Moungsrijun, Procedia Manuf., 2015, 2, 108-112.

261 P. Song, L. Shen, L. Zheng, K. Liu, W. Tian, J. Chen, Y. Luo, C. Tian, L. Xie and Z. Wei, Nano Select, 2021, 1-9.

262 H. Tang, Q. Cao, Z. He, S. Wang, J. Han, T. Li, B. Gao, J. Yang, D. Deng and X. Li, Sol. RRL, 2020, 4, 1900415.

263 G. Bai, Z. Wu, J. Li, T. Bu, W. Li, W. Li, F. Huang, Q. Zhang, Y.-B. Cheng and J. Zhong, Sol. Energy, 2019, 183, 306-314.

264 Y. Guo, H. Lei, C. Wang, J. Ma, C. Chen, X. Zheng, G. Yang, L. Xiong and Z. Tan, Sol. RRL, 2020, 4, 1900482.

265 G. Kumar, J. Kaur and R. Basu, Silicon, 2020, 73, DOI: 10.1007/s12633-020-00820-8.

266 J. Song, E. Zheng, J. Bian, X.-F. Wang, W. Tian, Y. Sanehira and T. Miyasaka, J. Mater. Chem. A, 2015, 3, 10837-10844.

267 J. Dagar, S. Castro-Hermosa, M. Gasbarri, A. L. Palma, L. Cina, F. Matteocci, E. Calabrò, A. Di Carlo and T. M. Brown, Nano Res., 2018, 11, 2669-2681.

268 W. Ke, D. Zhao, A. J. Cimaroli, C. R. Grice, P. Qin, Q. Liu, L. Xiong, Y. Yan and G. Fang, J. Mater. Chem. A, 2015, 3, 24163-24168.

269 L. Huang, X. Sun, C. Li, J. Xu, R. Xu, Y. Du, J. Ni, H. Cai, J. Li, Z. Hu and J. Zhang, ACS Appl. Mater. Interfaces, 2017, 9, 21909-21920.

270 G. Murugadoss, H. Kanda, S. Tanaka, H. Nishino, S. Ito, H. Imahori and T. Umeyama, J. Power Sources, 2016, 307, 891-897.

271 C. Zhang, S. Wang, H. Zhang, Y. Feng, W. Tian, Y. Yan, J. Bian, Y. Wang, S. Jin, S. M. Zakeeruddin, M. Grätzel and Y. Shi, Energy Environ. Sci., 2019, 12, 3585-3594.

272 J. Duan, Q. Xiong, B. Feng, Y. Xu, J. Zhang and H. Wang, Appl. Surf. Sci., 2017, 391, 677-683. 
273 P. Pinpithak, H.-W. Chen, A. Kulkarni, Y. Sanehira, M. Ikegami and T. Miyasaka, Chem. Lett., 2017, 46, 382-384. 274 Q. Dong, Y. Shi, K. Wang, Y. Li, S. Wang, H. Zhang, Y. Xing, Y. Du, X. Bai and T. Ma, J. Phys. Chem. C, 2015, 119, 1021210217.

275 Q. Jiang, Z. Chu, P. Wang, X. Yang, H. Liu, Y. Wang, Z. Yin, J. Wu, X. Zhang and J. You, Adv. Mater., 2017, 29, 1703852. 276 G. Yang, C. Chen, F. Yao, Z. Chen, Q. Zhang, X. Zheng, J. Ma, H. Lei, P. Qin, L. Xiong, W. Ke, G. Li, Y. Yan and G. Fang, Adv. Mater., 2018, 30, 1706023.

277 L. Xiong, M. Qin, C. Chen, J. Wen, G. Yang, Y. Guo, J. Ma, Q. Zhang, P. Qin, S. Li and G. Fang, Adv. Funct. Mater., 2018, 28, 1706276.

278 B. Roose, J.-P. C. Baena, K. C. Gödel, M. Graetzel, A. Hagfeldt, U. Steiner and A. Abate, Nano Energy, 2016, 30, 517-522.

279 Q. Liu, M.-C. Qin, W.-J. Ke, X.-L. Zheng, Z. Chen, P.-L. Qin, L.-B. Xiong, H.-W. Lei, J.-W. Wan, J. Wen, G. Yang, J.-J. Ma, Z.-Y. Zhang and G.-J. Fang, Adv. Funct. Mater., 2016, 26, 6069-6075.

280 X. Ren, D. Yang, Z. Yang, J. Feng, X. Zhu, J. Niu, Y. Liu, W. Zhao and S. F. Liu, ACS Appl. Mater. Interfaces, 2017, 9, 2421-2429.

281 W.-Q. Wu, D. Chen, Y.-B. Cheng and R. A. Caruso, Sol. RRL, 2017, 1, 1700117.

282 Z. Chen, G. Yang, X. Zheng, H. Lei, C. Chen, J. Ma, H. Wang and G. Fang, J. Power Sources, 2017, 351, 123-129.

283 T. Bu, X. Liu, Y. Zhou, J. Yi, X. Huang, L. Luo, J. Xiao, Z. Ku, Y. Peng, F. Huang, Y.-B. Cheng and J. Zhong, Energy Environ. Sci., 2017, 10, 2509-2515.

284 F. Guo, X. Sun, B. Liu, Z. Yang, J. Wei and D. Xu, Angew. Chem., Int. Ed., 2019, 58, 18460-18465.

285 W. Zhang, Y. Li, X. Liu, D. Tang, X. Li and X. Yuan, Chem. Eng. J., 2020, 379, 122298.

286 A. S. Subbiah, N. Mathews, S. Mhaisalkar and S. K. Sarkar, ACS Energy Lett., 2018, 3, 1482-1491.

287 L. Qiu, Z. Liu, L. K. Ono, Y. Jiang, D.-Y. Son, Z. Hawash, S. He and Y. Qi, Adv. Funct. Mater., 2019, 29, 1806779.

288 P. F. Méndez, S. K. M. Muhammed, E. M. Barea, S. Masi and I. Mora-Seró, Sol. RRL, 2019, 3, 1900191.

289 Y. Sui, C. A. Zorman and R. M. Sankaran, Plasma Processes Polym., 2020, 17, 2000009.

290 A. Dey, A. Chroneos, N. S. J. Braithwaite, R. P. Gandhiraman and S. Krishnamurthy, Appl. Phys. Rev., 2016, 3, 021301.

291 T. Minami, H. Nanto and S. Takata, Jpn. J. Appl. Phys., 1988, 27, L287-L289.

292 M. Ruske, G. Bräuer, J. Pistner, U. Pfäfflin and J. Szczyrbowski, Thin Solid Films, 1999, 351, 146-150.

293 F. Hellegouarc'h, F. Arefi-Khonsari, R. Planade and J. Amouroux, Sens. Actuators, B, 2001, 73, 27-34.

294 M. J. Tarlov and J. F. Evans, Chem. Mater., 1990, 2, 49-60.

295 H. Wang and A. L. Rogach, Chem. Mater., 2014, 26, 123-133.

296 N. Ren, J. Zhu, P. Shi, Q. Shan, T. Li, C. Wei, Y. Zhao and X. Zhang, Sol. Energy, 2018, 171, 907-913.

297 P. Tang, C. Liu, J. Zhang, L. Wu, W. Li, L. Feng, G. Zeng and W. Wang, Appl. Surf. Sci., 2018, 436, 134-140.
298 L. Lin, T. W. Jones, J. T.-W. Wang, A. Cook, N. D. Pham, N. W. Duffy, B. Mihaylov, M. Grigore, K. F. Anderson, B. C. Duck, H. Wang, J. Pu, J. Li, B. Chi and G. J. Wilson, Small, 2020, 16, 1901466.

299 V.-D. Dao, L. L. Larina and H.-S. Choi, Thin Solid Films, 2015, 593, 10-16.

300 C. Wang, D. Zhao, C. R. Grice, W. Liao, Y. Yu, A. Cimaroli, N. Shrestha, P. J. Roland, J. Chen, Z. Yu, P. Liu, N. Cheng, R. J. Ellingson, X. Zhao and Y. Yan, J. Mater. Chem. A, 2016, 4, 12080-12087.

301 Y. Kuang, V. Zardetto, R. van Gils, S. Karwal, D. Koushik, M. A. Verheijen, L. E. Black, C. Weijtens, S. Veenstra, R. Andriessen, W. M. M. Kessels and M. Creatore, ACS Appl. Mater. Interfaces, 2018, 10, 30367-30378.

302 T. Hu, T. Becker, N. Pourdavoud, J. Zhao, K. O. Brinkmann, R. Heiderhoff, T. Gahlmann, Z. Huang, S. Olthof, K. Meerholz, D. Többens, B. Cheng, Y. Chen and T. Riedl, Adv. Mater., 2017, 29, 1606656.

303 H. Yu, H.-I. Yeom, J. W. Lee, K. Lee, D. Hwang, J. Yun, J. Ryu, J. Lee, S. Bae, S. K. Kim and J. Jang, Adv. Mater., 2018, 30, 1704825.

304 J. A. Smith, O. S. Game, J. E. Bishop, E. L. K. Spooner, R. C. Kilbride, C. Greenland, R. Jayaprakash, T. I. Alanazi, E. J. Cassella, A. Tejada, G. Chistiakova, M. WongStringer, T. J. Routledge, A. J. Parnell, D. B. Hammond and D. G. Lidzey, ACS Appl. Energy Mater., 2020, 3, 55525562.

305 P. Chetri and J. C. Dhar, Semicond. Sci. Technol., 2020, 35, 045014.

306 W. Yuan, X. Liu, Z. Fang, H. Ning, X. Zhang, Y. Deng, P. Deng, Z. Liang, R. Yao and J. Peng, Mol. Cryst. Liq. Cryst., 2018, 676, 44-49.

307 C. Wang, C. Xiao, Y. Yu, D. Zhao, R. A. Awni, C. R. Grice, K. Ghimire, I. Constantinou, W. Liao, A. J. Cimaroli, P. Liu, J. Chen, N. J. Podraza, C.-S. Jiang, M. M. Al-Jassim, X. Zhao and Y. Yan, Adv. Energy Mater., 2017, 7, 1700414.

308 Q. Wang, C. Peng, L. Du, H. Li, W. Zhang, J. Xie, H. Qi, Y. Li, L. Tian and Y. Huang, Adv. Mater. Interfaces, 2020, 7, 1901866.

309 R. F. Service, Science, 2018, 360, 1386.

310 C. J. Traverse, R. Pandey, M. C. Barr and R. R. Lunt, Nat. Energy, 2017, 2, 849-860.

311 E. Della Gaspera, Y. Peng, Q. Hou, L. Spiccia, U. Bach, J. J. Jasieniak and Y.-B. Cheng, Nano Energy, 2015, 13, 249-257.

312 F. Huang, Y. Dkhissi, W. Huang, M. Xiao, I. Benesperi, S. Rubanov, Y. Zhu, X. Lin, L. Jiang, Y. Zhou, A. GrayWeale, J. Etheridge, C. R. McNeill, R. A. Caruso, U. Bach, L. Spiccia and Y.-B. Cheng, Nano Energy, 2014, 10, 10-18.

313 G. E. Eperon, D. Bryant, J. Troughton, S. D. Stranks, M. B. Johnston, T. Watson, D. A. Worsley and H. J. Snaith, J. Phys. Chem. Lett., 2015, 6, 129-138.

314 G. E. Eperon, V. M. Burlakov, A. Goriely and H. J. Snaith, ACS Nano, 2014, 8, 591-598.

315 M. B. Upama, M. Wright, N. K. Elumalai, M. A. Mahmud, D. Wang, K. H. Chan, C. Xu, F. Haque and A. Uddin, Curr. Appl. Phys., 2017, 17, 298-305. 
316 A. C. Véron, H. Zhang, A. Linden, F. Nüesch, J. Heier, R. Hany and T. Geiger, Org. Lett., 2014, 16, 1044-1047.

317 N. C. Davy, M. Sezen-Edmonds, J. Gao, X. Lin, A. Liu, N. Yao, A. Kahn and Y.-L. Loo, Nat. Energy, 2017, 2, 17104.

318 N. C. Davy, G. Man, R. A. Kerner, M. A. Fusella, G. E. Purdum, M. Sezen, B. P. Rand, A. Kahn and Y.-L. Loo, Chem. Mater., 2016, 28, 673-681.

319 D. Liu, C. Yang and R. R. Lunt, Joule, 2018, 2, 1827-1837.

320 X. Zhang, L. Li, E. Fan, Q. Xue, Y. Bian, F. Wu and R. Chen, Chem. Soc. Rev., 2018, 47, 7239-7302.

321 A. Noori, M. F. El-Kady, M. S. Rahmanifar, R. B. Kaner and M. F. Mousavi, Chem. Soc. Rev., 2019, 48, 1272-1341.

322 D. P. Dubal, O. Ayyad, V. Ruiz and P. Gómez-Romero, Chem. Soc. Rev., 2015, 44, 1777-1790.

323 D. P. Dubal, N. R. Chodankar, D.-H. Kim and P. GomezRomero, Chem. Soc. Rev., 2018, 47, 2065-2129.

324 T. Kim, W. Song, D.-Y. Son, L. K. Ono and Y. Qi, J. Mater. Chem. A, 2019, 7, 2942-2964.

325 F. Cheng, J. Liang, Z. Tao and J. Chen, Adv. Mater., 2011, 23, 1695-1715.

326 M. M. Thackeray, C. Wolverton and E. D. Isaacs, Energy Environ. Sci., 2012, 5, 7854-7863.

327 J. Hou, Y. Shao, M. W. Ellis, R. B. Moore and B. Yi, Phys. Chem. Chem. Phys., 2011, 13, 15384-15402.

328 B. J. Landi, M. J. Ganter, C. D. Cress, R. A. DiLeo and R. P. Raffaelle, Energy Environ. Sci., 2009, 2, 638-654.

329 M. Ge, J. Rong, X. Fang and C. Zhou, Nano Lett., 2012, 12, 2318-2323.

330 J. Jiang, Y. Li, J. Liu, X. Huang, C. Yuan and X. W. Lou, Adv. Mater., 2012, 24, 5166-5180.

331 Q. Su, S. Wang, M. Feng, G. Du and B. Xu, Sci. Rep., 2017, 7, 7275.

332 T. Stephenson, Z. Li, B. Olsen and D. Mitlin, Energy Environ. Sci., 2014, 7, 209-231.

333 C. Jiang, C. Yuan, P. Li, H.-g. Wang, Y. Li and Q. Duan, J. Mater. Chem. A, 2016, 4, 7251-7256.

334 Y. Jiang, Z.-J. Jiang, L. Yang, S. Cheng and M. Liu, J. Mater. Chem. A, 2015, 3, 11847-11856.

335 C. Liang, T. Zhai, W. Wang, J. Chen, W. Zhao, X. Lu and Y. Tong, J. Mater. Chem. A, 2014, 2, 7214-7220.

336 S. Yang, X. Feng, S. Ivanovici and K. Müllen, Angew. Chem., Int. Ed., 2010, 49, 8408-8411.

337 Q. Wu, Q. Shao, Q. Li, Q. Duan, Y. Li and H.-g. Wang, ACS Appl. Mater. Interfaces, 2018, 10, 15642-15651.

338 H.-g. Wang, Q. Wu, Y. Wang, X. Wang, L. Wu, S. Song and H. Zhang, Adv. Energy Mater., 2019, 9, 1802993.

339 H. Mou, W. Xiao, C. Miao, R. Li and L. Yu, Front. Chem., 2020, 8, 141.

340 F. Zoller, D. Böhm, T. Bein and D. Fattakhova-Rohlfing, ChemSusChem, 2019, 12, 4140-4159.

341 H.-g. Wang, C. Jiang, C. Yuan, Q. Wu, Q. Li and Q. Duan, Chem. Eng. J., 2018, 332, 237-244.

342 N. Li, H. Song, H. Cui and C. Wang, Nano Energy, 2014, 3, 102-112.

343 M. A. Kebede, Curr. Opin. Electrochem., 2020, 21, 182-187.

344 Y. Idota, T. Kubota, A. Matsufuji, Y. Maekawa and T. Miyasaka, Science, 1997, 276, 1395-1397.
345 J. Y. Huang, L. Zhong, C. M. Wang, J. P. Sullivan, W. Xu, L. Q. Zhang, S. X. Mao, N. S. Hudak, X. H. Liu, A. Subramanian, H. Fan, L. Qi, A. Kushima and J. Li, Science, 2010, 330, 1515-1520.

346 L. Noerochim, J.-Z. Wang, S.-L. Chou, H.-J. Li and H.-K. Liu, Electrochim. Acta, 2010, 56, 314-320.

347 Y.-T. Liu, P. Zhang, N. Sun, B. Anasori, Q.-Z. Zhu, H. Liu, Y. Gogotsi and B. Xu, Adv. Mater., 2018, 30, 1707334.

348 J. Mei, T. Liao, L. Kou and Z. Sun, Adv. Mater., 2017, 29, 1700176.

349 Y. Zhao, L. P. Wang, M. T. Sougrati, Z. Feng, Y. Leconte, A. Fisher, M. Srinivasan and Z. Xu, Adv. Energy Mater., 2017, 7, 1601424.

350 J. Wang, W. Li, F. Wang, Y. Xia, A. M. Asiri and D. Zhao, Nanoscale, 2014, 6, 3217-3222.

351 C. Miao, M. Liu, Y.-B. He, X. Qin, L. Tang, B. Huang, R. Li, B. Li and F. Kang, Energy Storage Mater., 2016, 3, 98-105.

352 S. Wang, Y. Yang, C. Jiang, Y. Hong, W. Quan, Z. Zhang and Z. Tang, J. Mater. Chem. A, 2016, 4, 12714-12719.

353 K. Zhao, L. Zhang, R. Xia, Y. Dong, W. Xu, C. Niu, L. He, M. Yan, L. Qu and L. Mai, Small, 2016, 12, 588-594.

354 D. Zhou, W.-L. Song and L.-Z. Fan, ACS Appl. Mater. Interfaces, 2015, 7, 21472-21478.

355 C. Zhu, S. Zhu, K. Zhang, Z. Hui, H. Pan, Z. Chen, Y. Li, D. Zhang and D.-W. Wang, Sci. Rep., 2016, 6, 25829.

356 W. Yao, S. Wu, L. Zhan and Y. Wang, Chem. Eng. J., 2019, 361, 329-341.

357 N. Wu, W. Du, X. Gao, L. Zhao, G. Liu, X. Liu, H. Wu and Y.-B. He, Nanoscale, 2018, 10, 11460-11466.

358 J. Liang, C. Yuan, H. Li, K. Fan, Z. Wei, H. Sun and J. Ma, Nano-Micro Lett., 2017, 10, 21.

359 L. Xia, S. Wang, G. Liu, L. Ding, D. Li, H. Wang and S. Qiao, Small, 2016, 12, 853-859.

360 M. Wang, X. Feng, H. Xiang, Y. Feng, C. Qin, P. Yan and Y. Yu, Small Methods, 2019, 3, 1900355.

361 Z.-M. Luo, Y.-G. Sun and H.-Y. Liu, Chin. Chem. Lett., 2015, 26, 1403-1408.

362 F. Ma, F. Geng, A. Yuan and J. Xu, Phys. Chem. Chem. Phys., 2017, 19, 9983-9991.

363 Y. Xiang, W. Zhu, W. Qiu, W. Guo, J. Lei, D. Liu, D. Qu, Z. Xie, H. Tang and J. Li, ChemistrySelect, 2018, 3, 911-916.

364 J. Balach, J. Linnemann, T. Jaumann and L. Giebeler, J. Mater. Chem. A, 2018, 6, 23127-23168.

365 J. Liu, L. Yuan, K. Yuan, Z. Li, Z. Hao, J. Xiang and Y. Huang, Nanoscale, 2016, 8, 13638-13645.

366 H. Yu, D. Byun and J. K. Lee, Appl. Surf. Sci., 2018, 461, 154160.

367 M. Wang, L. Fan, X. Wu, D. Tian, J. Cheng, Y. Qiu, H. Wu, B. Guan, N. Zhang, K. Sun and Y. Wang, J. Mater. Chem. A, 2017, 5, 19613-19618.

368 Q. Liu, Q. Jiang, L. Jiang, J. Peng, Y. Gao, Z. Duan and X. Lu, Appl. Surf. Sci., 2018, 462, 393-398.

369 L. I. Rui, S. Xiaogang, H. Yapan, Z. Jingyi, H. Qiang and X. Yuhao, Vacuum, 2019, 168, 108820.

370 Y. Wu, W. Zhang, T. Han, Z. Shen, D. Cheng, H. Zhang, J. Li, H. Zhang and J. Liu, Appl. Surf. Sci., 2019, 489, 462-469. 
371 Q. Xiao, K. Wang, X. Wang, S. Huang, N. Cai and N. Li, Mater. Chem. Phys., 2020, 239, 122070.

372 M. Qi, X. Liang, F. Wang, M. Han, J. Yin and M. Chen, J. Alloys Compd., 2019, 799, 345-350.

373 N. Hu, X. Lv, Y. Dai, L. Fan, D. Xiong and X. Li, ACS Appl. Mater. Interfaces, 2018, 10, 18665-18674.

374 C. K. Balavigneswaran, R. Venkatesan, P. S. Karuppiah, G. Kumar, P. Paliwal, S. Krishnamurthy, B. Kadalmani, S. K. Mahto and N. Misra, ACS Appl. Bio Mater., 2020, 3, 197-207.

375 B. Cao, D. Li, B. Hou, Y. Mo, L. Yin and Y. Chen, ACS Appl. Mater. Interfaces, 2016, 8, 27795-27802.

376 X. Li, Y. Lu, Z. Hou, W. Zhang, Y. Zhu, Y. Qian, J. Liang and Y. Qian, ACS Appl. Mater. Interfaces, 2016, 8, 19550-19557.

377 Y.-Z. Zhang, Y. Wang, T. Cheng, L.-Q. Yao, X. Li, W.-Y. Lai and W. Huang, Chem. Soc. Rev., 2019, 48, 3229-3264.

378 Y. Shao, M. F. El-Kady, J. Sun, Y. Li, Q. Zhang, M. Zhu, H. Wang, B. Dunn and R. B. Kaner, Chem. Rev., 2018, 118, 9233-9280.

379 J. Park, D. B. Ahn, J. Kim, E. Cha, B.-S. Bae, S.-Y. Lee and J.-U. Park, Sci. Adv., 2019, 5, eaay0764.

380 H. Li and J. Liang, Adv. Mater., 2020, 32, 2070023.

381 J. Sun, B. Cui, F. Chu, C. Yun, M. He, L. Li and Y. Song, Nanomaterials, 2018, 8, 528.

382 M. Areir, Y. Xu, D. Harrison and J. Fyson, Mater. Sci. Eng., B, 2017, 226, 29-38.

383 K. Shen, H. Mei, B. Li, J. Ding and S. Yang, Adv. Energy Mater., 2018, 8, 1701527.

384 K.-H. Choi, J. Yoo, C. K. Lee and S.-Y. Lee, Energy Environ. Sci., 2016, 9, 2812-2821.

385 M. A. A. Mohd Abdah, N. H. N. Azman, S. Kulandaivalu and Y. Sulaiman, Mater. Des., 2020, 186, 108199.

386 C. An, Y. Zhang, H. Guo and Y. Wang, Nanoscale Adv., 2019, 1, 4644-4658.

387 S. Najib and E. Erdem, Nanoscale Adv., 2019, 1, 2817-2827.

388 X. Hong, S. Li, R. Wang and J. Fu, J. Alloys Compd., 2019, 775, 15-21.

389 R. Sahoo, D. T. Pham, T. H. Lee, T. H. T. Luu, J. Seok and Y. H. Lee, ACS Nano, 2018, 12, 8494-8505.

390 T. Xiong, T. L. Tan, L. Lu, W. S. V. Lee and J. Xue, Adv. Energy Mater., 2018, 8, 1702630.

391 L. Huang, D. Chen, Y. Ding, S. Feng, Z. L. Wang and M. Liu, Nano Lett., 2013, 13, 3135-3139.

392 T. Liu, L. Zhang, W. You and J. Yu, Small, 2018, 14, 1702407. 393 S. Sheng, W. Liu, K. Zhu, K. Cheng, K. Ye, G. Wang, D. Cao and J. Yan, J. Colloid Interface Sci., 2019, 536, 235-244.

394 J.-C. Lee, A.-I. Gopalan, G. Saianand, K.-P. Lee and W.-J. Kim, Nanomaterials, 2020, 10, 456.

395 G. Saianand, A.-I. Gopalan, J.-C. Lee, C. Sathish, K. Gopalakrishnan, G. E. Unni, D. Shanbhag, V. D. B. C. Dasireddy, J. Yi, S. Xi, A. H. Al-Muhtaseb and A. Vinu, Small, 2020, 16, 1903937.

396 X. Lang, G. Saianand, W. Fu and S. Ramakrishna, Bull. Chem. Soc. Jpn., 2021, 94, 8-20.

397 M. R. Benzigar, S. Joseph, G. Saianand, A.-I. Gopalan, S. Sarkar, S. Srinivasan, D.-H. Park, S. Kim,
S. N. Talapaneni, K. Ramadass and A. Vinu, Microporous Mesoporous Mater., 2019, 285, 21-31.

398 J.-C. Lee, A.-I. Gopalan, G. Sai-Anand, K.-P. Lee and W.-J. Kim, Catalysts, 2019, 9, 170.

399 T. Sridara, J. Upan, G. Saianand, A. Tuantranont, C. Karuwan and J. Jakmunee, Sensors, 2020, 20, 808.

400 M. Rashmi, R. Padmanaban, V. Karthikeyan, V. A. L. Roy, A.-I. Gopalan, G. Saianand, W.-J. Kim and V. Kannan, Catalysts, 2020, 10, 34.

401 Y. Zhang, Z. Hu, Y. Liang, Y. Yang, N. An, Z. Li and H. Wu, J. Mater. Chem. A, 2015, 3, 15057-15067.

402 F. Li, J. Song, H. Yang, S. Gan, Q. Zhang, D. Han, A. Ivaska and L. Niu, Nanotechnology, 2009, 20, 455602.

403 A. Numan, N. Duraisamy, F. Saiha Omar, Y. K. Mahipal, K. Ramesh and S. Ramesh, RSC Adv., 2016, 6, 34894-34902.

404 D. Zhao, M. Dai, Y. Tong, X. Song and X. Wu, CrystEngComm, 2019, 21, 5789-5796.

405 Q. Liao and C. Wang, CrystEngComm, 2019, 21, 662-672.

406 M. Cao, W. Cheng, X. Ni, Y. Hu and G. Han, Electrochim. Acta, 2020, 345, 136172.

407 Y. Li, G. Wang, T. Wei, Z. Fan and P. Yan, Nano Energy, 2016, 19, 165-175.

408 Y. Kang, Z. Li, K. Xu, X. He, S. Wei and Y. Cao, J. Alloys Compd., 2019, 779, 728-734.

409 P. Asen, M. Haghighi, S. Shahrokhian and N. Taghavinia, J. Alloys Compd., 2019, 782, 38-50.

410 Y. Luan, G. Nie, X. Zhao, N. Qiao, X. Liu, H. Wang, X. Zhang, Y. Chen and Y.-Z. Long, Electrochim. Acta, 2019, 308, 121130.

411 X. Zhang, Q. Huang, M. Zhang, M. Li, J. Hu and G. Yuan, J. Alloys Compd., 2020, 822, 153718.

412 A. Ali, M. Ammar, Z. Yahya, M. Waqas, M. A. Jamal and E. H. M. Salhabi, New J. Chem., 2019, 43, 10583-10589.

413 Z. Wang, Y. Long, D. Cao, D. Han and F. Gu, Electrochim. Acta, 2019, 307, 341-350.

414 Y. M. Dai, S. C. Tang, J. Q. Peng, H. Y. Chen, Z. X. Ba, Y. J. Ma and X. K. Meng, Mater. Lett., 2014, 130, 107-110.

415 X. Meng, M. Zhou, X. Li, J. Yao, F. Liu, H. He, P. Xiao and Y. Zhang, Electrochim. Acta, 2013, 109, 20-26.

416 W. Wang, Q. Hao, W. Lei, X. Xia and X. Wang, RSC Adv., 2012, 2, 10268-10274.

417 J. Ge, Y. Qu, L. Cao, F. Wang, L. Dou, J. Yu and B. Ding, J. Mater. Chem. A, 2016, 4, 7795-7804.

418 J. Mu, B. Chen, Z. Guo, M. Zhang, Z. Zhang, C. Shao and Y. Liu, J. Colloid Interface Sci., 2011, 356, 706-712.

419 L.-L. Hu, L.-P. Yang, D. Zhang, X.-S. Tao, C. Zeng, A.-M. Cao and L.-J. Wan, Chem. Commun., 2017, 53, 11189-11192.

420 R. Barik, V. Tanwar, R. Kumar and P. P. Ingole, J. Mater. Chem. A, 2020, 8, 15110-15121.

421 K.-K. Liu, Q. Jiang, C. Kacica, H. G. Derami, P. Biswas and S. Singamaneni, RSC Adv., 2018, 8, 31296-31302.

422 S. Biswas, V. Sharma, D. Mandal, A. Chowdhury, M. Chakravarty, S. Priya, C. C. Gowda, P. De, I. Singh and A. Chandra, CrystEngComm, 2020, 22, 1633-1644.

423 M. Zhu, Y. Huang, Y. Huang, W. Meng, Q. Gong, G. Li and C. Zhi, J. Mater. Chem. A, 2015, 3, 21321-21327. 
424 S. Y. Kim, T. Y. Yun, K. S. Yu and H. C. Moon, ACS Appl. Mater. Interfaces, 2020, 12, 51978-51986.

425 Q. Guo, X. Zhao, Z. Li, D. Wang and G. Nie, Chem. Eng. J., 2020, 384, 123370.

426 K.-W. Kim, T. Y. Yun, S.-H. You, X. Tang, J. Lee, Y. Seo, Y.-T. Kim, S. H. Kim, H. C. Moon and J. K. Kim, NPG Asia Mater., 2020, 12, 84.

427 M.-H. Jo, B.-R. Koo and H.-J. Ahn, Ceram. Int., 2020, 46, 25066-25072.

428 J.-h. Zhang, J.-p. Tu, D. Zhou, H. Tang, L. Li, X.-l. Wang and C.-d. Gu, J. Mater. Chem. C, 2014, 2, 10409-10417.

429 U. zum Felde, M. Haase and H. Weller, J. Phys. Chem. B, 2000, 104, 9388-9395.

430 K.-H. Kim, B.-R. Koo and H.-J. Ahn, Ceram. Int., 2019, 45, 15990-15995.

431 D. Cummins, G. Boschloo, M. Ryan, D. Corr, S. N. Rao and D. Fitzmaurice, J. Phys. Chem. B, 2000, 104, 11449-11459.

432 R. Goei, A. J. Ong, T. J. Hao, L. J. Yi, L. S. Kuang, D. Mandler, S. Magdassi and A. I. Yoong Tok, Ceram. Int., 2021, 47, 18433-18442.

433 T. D. Nguyen, L. P. Yeo, T. C. Kei, D. Mandler, S. Magdassi and A. I. Y. Tok, Adv. Opt. Mater., 2019, 7, 1801389.

434 H. Ha, M. Yoo, H. An, K. Shin, T. Han, Y. Sohn, S. Kim, S.-R. Lee, J. H. Han and H. Y. Kim, Sci. Rep., 2017, 7, 14427.

435 R. Shen, Y. Hong, J. J. Stankovich, Z. Wang, S. Dai and X. Jin, J. Mater. Chem. A, 2015, 3, 17635-17643.

436 H. Yu, J. Li, Y. Tian and Z. Li, J. Alloys Compd., 2018, 765, 624-634.

437 H. J. Ko, Y. F. Chen, S. K. Hong, H. Wenisch, T. Yao and D. C. Look, Appl. Phys. Lett., 2000, 77, 3761-3763.

438 M.-C. Jun, S.-U. Park and J.-H. Koh, Nanoscale Res. Lett., 2012, 7, 639.

439 S. D. Ponja, S. Sathasivam, I. P. Parkin and C. J. Carmalt, Sci. Rep., 2020, 10, 638.

440 H. J. Al-Asedy and S. A. Al-khafaji, Appl. Phys. A: Mater. Sci. Process., 2020, 126, 701.

441 Z. Szabó, J. Volk, Z. E. Horváth, Z. Medveczky, Z. Czigány, K. Vad and Z. Baji, Mater. Sci. Semicond. Process., 2019, 101, 95-102.
442 J. Bruncko, P. Šutta, M. Netrvalová, M. Michalka and A. Vincze, Vacuum, 2019, 159, 134-140.

443 S.-M. Park, T. Ikegami and K. Ebihara, Thin Solid Films, 2006, 513, 90-94.

444 Q.-B. Ma, Z.-Z. Ye, H.-P. He, S.-H. Hu, J.-R. Wang, L.-P. Zhu, Y.-Z. Zhang and B.-H. Zhao, J. Cryst. Growth, 2007, 304, 6468.

445 V. Assunção, E. Fortunato, A. Marques, H. Águas, I. Ferreira, M. E. V. Costa and R. Martins, Thin Solid Films, 2003, 427, 401-405.

446 X. Yu, J. Ma, F. Ji, Y. Wang, C. Cheng and H. Ma, Appl. Surf. Sci., 2005, 245, 310-315.

447 T. Prasada Rao and M. C. Santhosh Kumar, J. Alloys Compd., 2010, 506, 788-793.

448 H. Gómez and M. d. l. L. Olvera, Mater. Sci. Eng., B, 2006, 134, 20-26.

449 L. Gong, J. Lu and Z. Ye, Sol. Energy Mater. Sol. Cells, 2010, 94, 937-941.

450 M. Miyazaki, K. Sato, A. Mitsui and H. Nishimura, J. NonCryst. Solids, 1997, 218, 323-328.

451 A. Tiburcio-Silver, A. Sanchez-Juarez and A. Avila-Garcia, Sol. Energy Mater. Sol. Cells, 1998, 55, 3-10.

452 C.-Y. Tsay, K.-S. Fan and C.-M. Lei, J. Alloys Compd., 2012, 512, 216-222.

453 H. Ohta, M. Orita, M. Hirano, H. Tanji, H. Kawazoe and H. Hosono, Appl. Phys. Lett., 2000, 76, 2740-2742.

454 J.-K. Sheu, Y. S. Lu, M.-L. Lee, W. C. Lai, C. H. Kuo and C.-J. Tun, Appl. Phys. Lett., 2007, 90, 263511.

455 T. Minami, T. Yamamoto and T. Miyata, Thin Solid Films, 2000, 366, 63-68.

456 A. R. Babar, P. R. Deshamukh, R. J. Deokate, D. Haranath, C. H. Bhosale and K. Y. Rajpure, J. Phys. D: Appl. Phys., 2008, 41, 135404.

457 M. Grundmann, H. Frenzel, A. Lajn, M. Lorenz, F. Schein and H. von Wenckstern, Phys. Status Solidi B, 2010, 207, 1437-1449. 